\title{
The Role of MicroRNAs in Human Nasopharyngeal Carcinoma
}

\author{
by \\ Jeffrey Bruce
}

A thesis submitted in conformity with the requirements

for the degree of Doctor of Philosophy

Graduate Department of Medical Biophysics

University of Toronto

(C) Jeffrey Bruce, 2014 


\title{
The Role of MicroRNAs in
}

\section{Human Nasopharyngeal Carcinoma}

\author{
Jeffrey Bruce \\ Doctor of Philosophy \\ Graduate Department of Medical Biophysics \\ University of Toronto
}

2014

\section{Abstract}

Despite significant improvement in locoregional control in the contemporary era, nasopharyngeal carcinoma (NPC) patients still suffer from a significant risk of distant metastasis (DM). Thus, developing methods to better identify and treat NPC patients at risk of DM is of paramount importance. MicroRNAs (miRNAs) have been shown to provide insight into both the biology and clinical behaviour of human cancers; hence, this thesis aims to characterize both the biological function and the prognostic utility of miRNAs in NPC.

First, miR-375 was identified as the most consistently and significantly down-regulated miRNA in head and neck cancer samples (including NPC) analyzed. Functional experiments confirmed a tumour suppressor role for miR-375. A novel target of miR-375, metadherin, was identified as a likely mediator of these effects; possibly through activation of the PI3K/Akt pathway. In addition, a clinical relationship was observed wherein NPC patients whose tumours expressed high metadherin transcript levels had an increased risk of developing distant metastases. 
Next, a 4-miRNA expression signature associated with risk of DM was identified in a training set of 125 NPC patients (HR 8.25; $\mathrm{p}<0.001)$. This signature was subsequently confirmed to be predictive of DM in an independent validation set $(n=121$, HR $3.2 ; p=0.01)$. Moreover, the 4-miRNA signature was observed to add to the prognostic value of the currently utilized clinical staging system. In addition, pathway enrichment analysis identified a potential role for these miRNAs in cell cycle regulation.

Finally, a pilot project was undertaken which established the feasibility and utility of molecular inversion probe (MIP) technology for the detection of copy-number alterations (CNAs) in small archival NPC biopsy samples.

In conclusion, the insights presented herein provide both clinical and biological insights into NPC, which will advance us closer to the goal of personalized management of this disease and improve treatment and outcome for future NPC patients. 


\section{Acknowledgments}

First, I would like to express my deep gratitude to my supervisor and mentor, Fei-Fei Liu for her continuous scientific, personal and professional guidance. Her enthusiasm for scientific discovery and constant focus on using these discoveries to impact patient care are what gave me purpose and kept me engaged during the long hours of bench work and data analysis. Her extensive network of fellow researchers and physicians, and confidence in my abilities (even when I sometimes lacked the same confidence in myself) also provided me with the opportunity to participate in many exciting collaborations.

In addition, I would like to thank the other members of my advisory committee, Lori Frappier and Laurie Ailles for their time and effort. Their thoughtful review of the progress of my project and valuable scientific advice was instrumental to my success as a $\mathrm{PhD}$ candidate.

I would also like to acknowledge the members of the Liu Lab; past and present. In particular, Angela Hui who was my mentor and colleague in much of the work presented in this thesis. Her extensive knowledge of nasopharyngeal carcinoma, biospecimen analysis, and cell biology was invaluable as I navigated the waters of translational research. A special thanks also to Emma Ito, Ronald Wu, Xiao Zhao, Lusia Sepiashvili, Christine How, Michelle Lenarduzzi, Kenneth Yip, Winnie Yue, Justin Williams, Mia Labitan, Carolina Lee, Nehad Alajez, Inki Kim, Takashi Kawanaka, Pierre-Antoine Bissey, and Willa Shi for their assistance and advice along the way.

I would also like to thank my friends; some of whom I've mentioned above, but also including Matt Myles, Mark Myles, Andrew Evans, Brad De Souza, Adam and Jasmine Kent, Fil and Iza Aleksanderek, Cole and Elizabeth Thorsen, Josh Gordon, Ian Morellato, Scott MacLennan, Kim Tobros, Rob Byczynski, Dave Correia, Frank Coscarelli, Meagen Resendes, Steve Amato and Deb and Jim Tobros.

I would like to thank my parents, Phillip and Alice Bruce; just knowing that I have their unconditional love and support have made every accomplishment in my life thus far, and those to come, possible. Thank you to my sisters Jennifer, Caroline, Cathy, and Susie for being a constant source of friendship, love and comfort. And special thanks to my nephew Tate, for being so strong and bringing so much joy into my life.

Finally, thank you to my girlfriend Melissa. You could say I owe my entire degree to her; it was only with her support and encouragement that I finally made the decision to change graduate departments entirely after seven months of a research project that didn't suit me, and follow my desire to do translational cancer research. More than that, she has been the most supportive, understanding, encouraging, and loving companion and friend a man could ask for. Thanks for being you, Melissa. 


\section{Table of Contents}

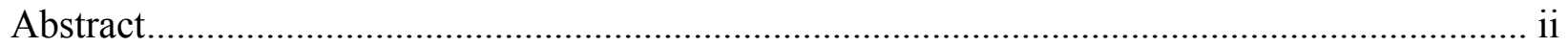

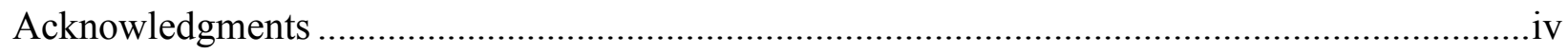

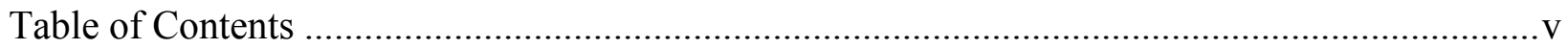

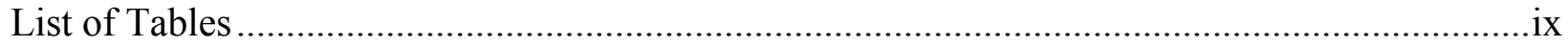

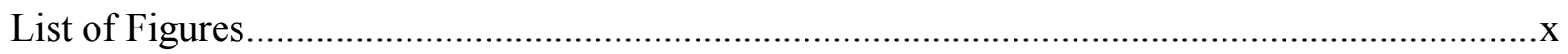

List of Abbreviations ................................................................................................. xii

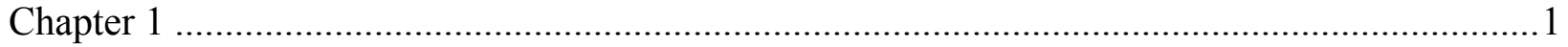

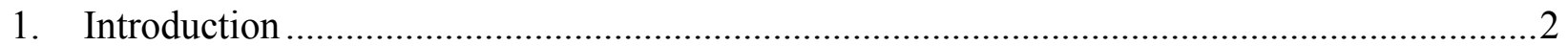

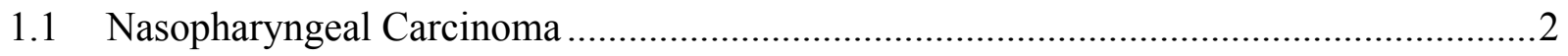

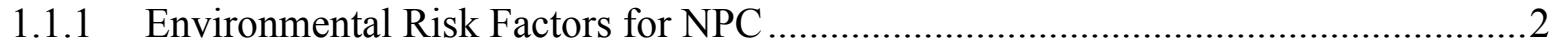

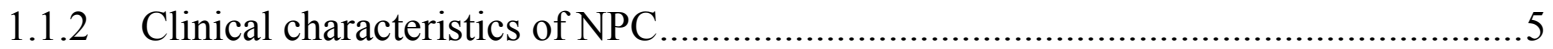

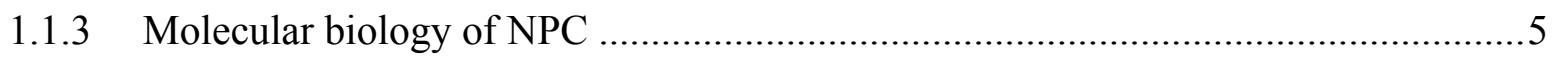

1.1.3.1 Genetic alterations in NPC ......................................................................

1.1.3.2 The role of EBV in NP tumourigenesis .....................................................10

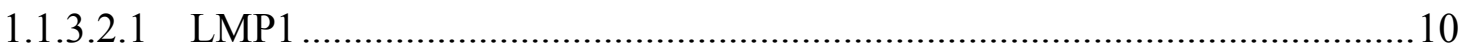

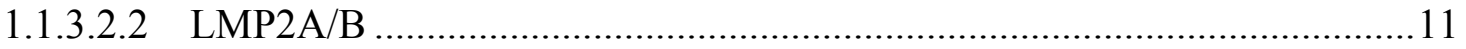

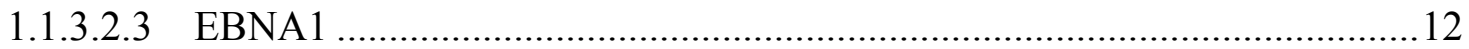

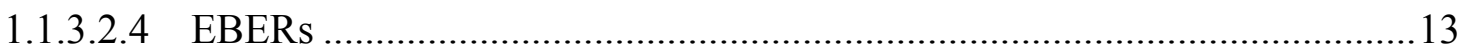

1.1.4 Prognostic/diagnostic biomarkers in NPC ............................................................ 14

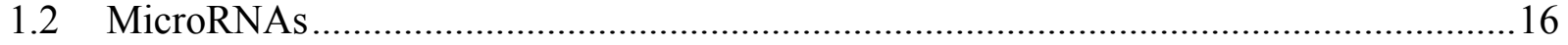

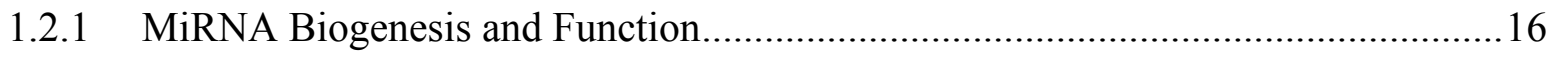

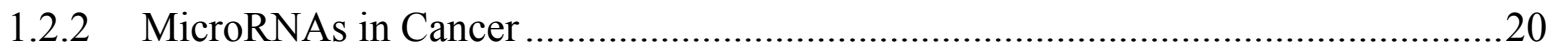

1.2.3 MicroRNA Expression Signatures in Cancer .....................................................20

1.2.4 Human MicroRNA Expression in Nasopharyngeal Carcinoma ................................21

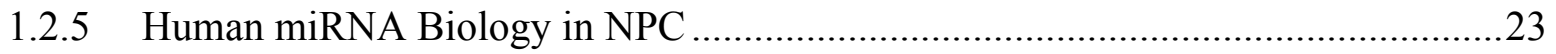

1.2.6 EBV encoded miRNAs in Nasopharyngeal Carcinoma ..........................................24

1.2.7 MicroRNA Expression Profiling Platforms …………..........................................25

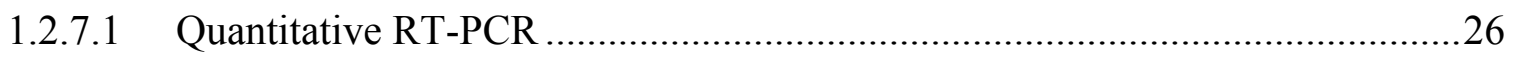

1.2.7.2 MiRNA Microarrays ………………………..........................................2

1.2.7.3 Digital molecular-barcoding expression analysis ............................................28 


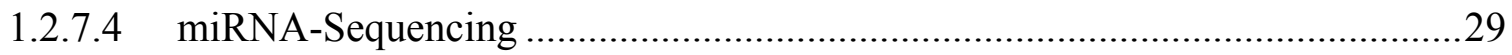

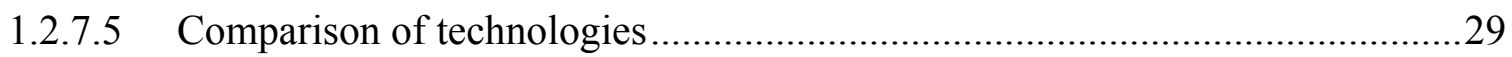

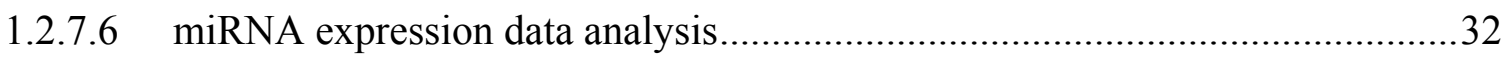

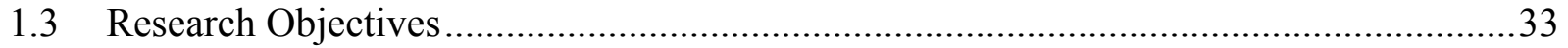

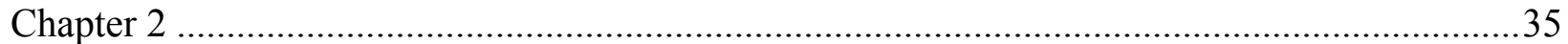

2. Significance of Dysregulated Metadherin and MicroRNA-375 in Head and Neck Cancer ..36

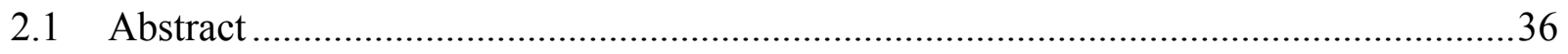

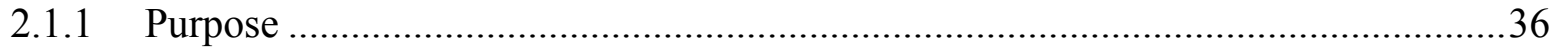

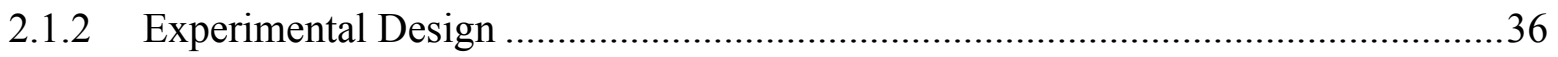

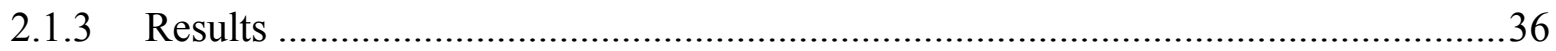

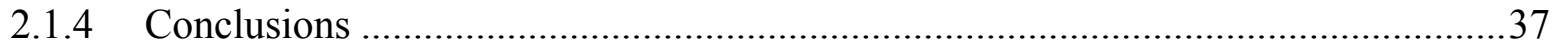

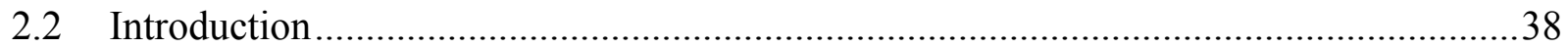

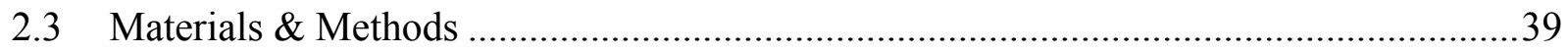

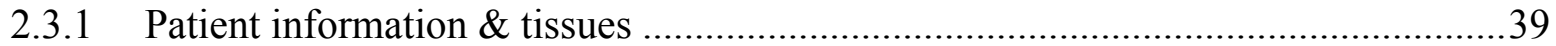

2.3.2 RNA purification from FFPE samples ………………........................................

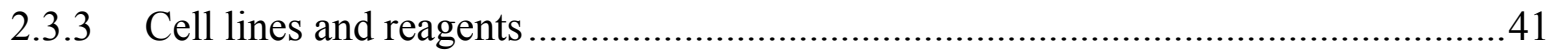

2.3.4 5-aza-2'-deoxycytidine treatment and quantitative $\mathrm{CpG}$ methylation analysis ........41

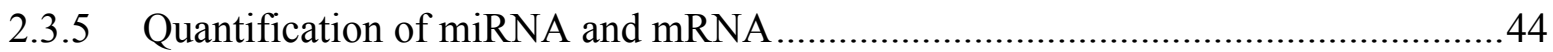

2.3.6 Construction of plasmids and luciferase assays .....................................................46

2.3.7 Viability and clonogenic assays ………..............................................................

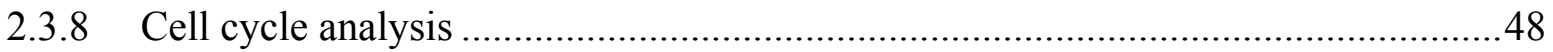

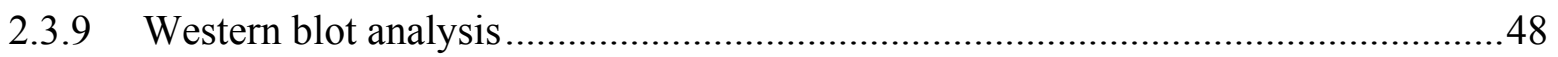

2.3.10 In vitro migration and invasion assays ................................................................49

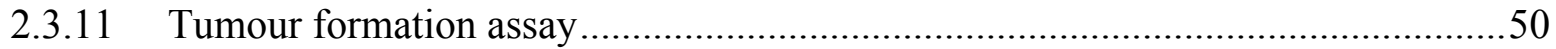

2.3.12 Immunohistochemical (IHC) detection of MTDH expression................................50

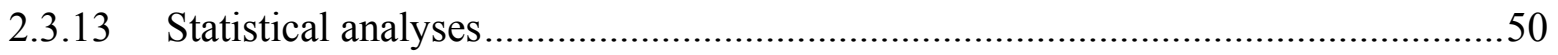

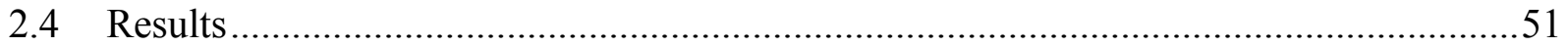

2.4.1 Down-regulation of miR-375 in HNC cell lines and primary NPC

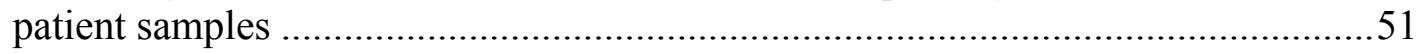

2.4.2 Methylation status of the genomic region containing miR-375 ............................56

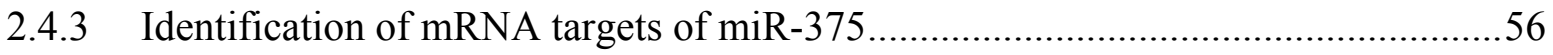


2.4.4 Effects of MTDH knock-down on HNC cells

2.4.5 Effects of miR-375 transfection and MTDH knock-down on PI(3)K-Akt signalling

2.4.6 MTDH knock-down and miR-375 transfection delayed tumour formation in-vivo.68

2.4.7 Over-expression of MTDH in primary HNC samples...............................................68

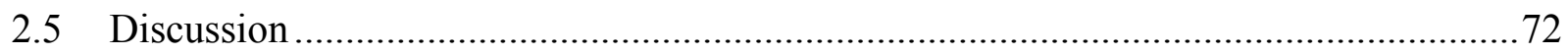

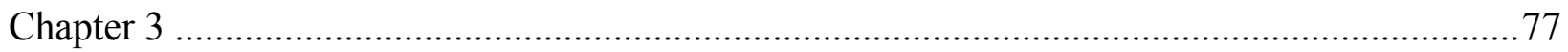

3. The Identification of a microRNA Signature Associated with Risk of Distant

Metastasis in Nasopharyngeal Carcinoma ........................................................................

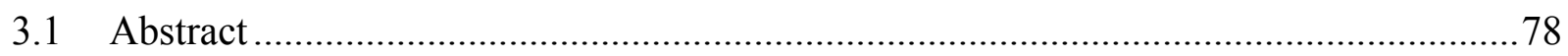

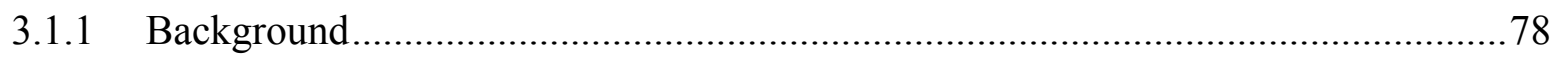

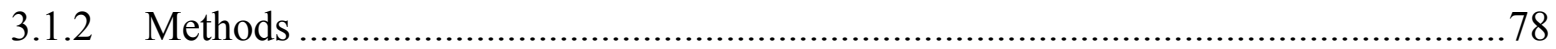

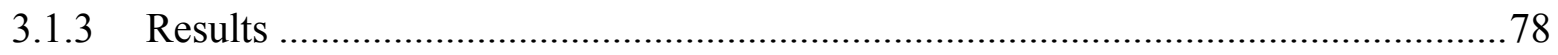

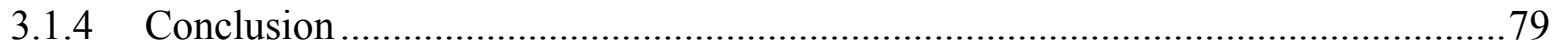

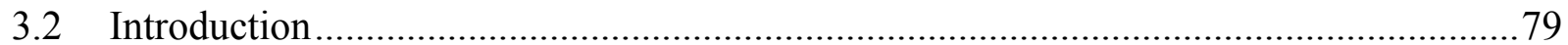

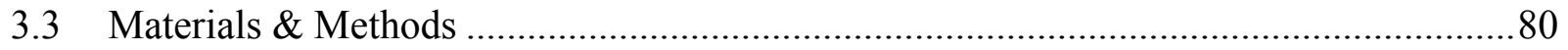

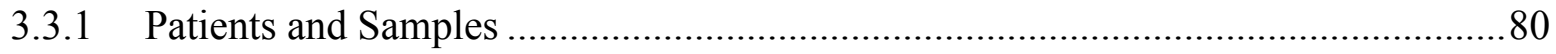

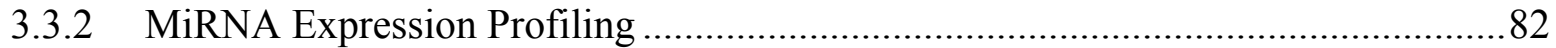

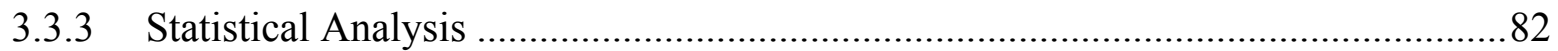

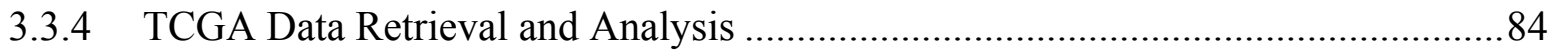

3.3.5 Target Identification and Pathway Analysis ..........................................................8

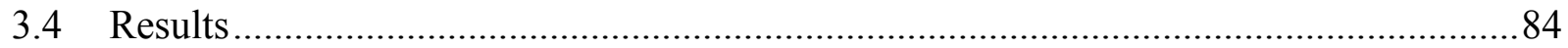

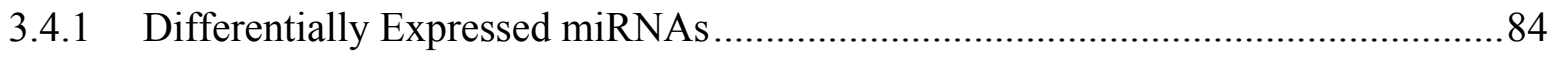

3.4.2 Generation and validation of miRNA signature associated with risk of

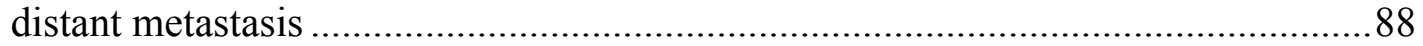

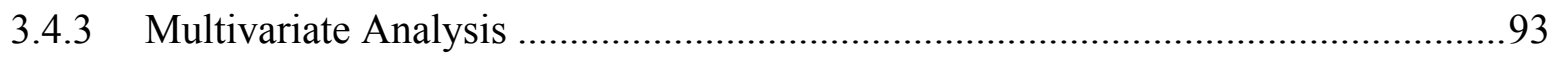

3.4.4 Validated miRNA Target Pathway Enrichment Analysis ..........................................99

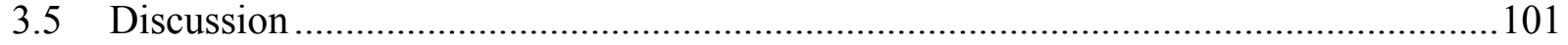

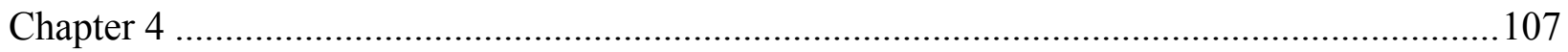

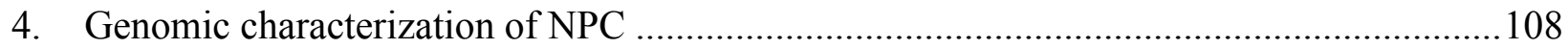

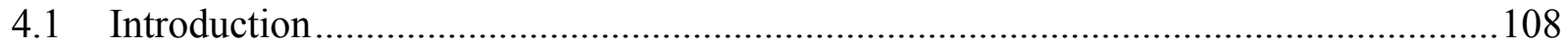

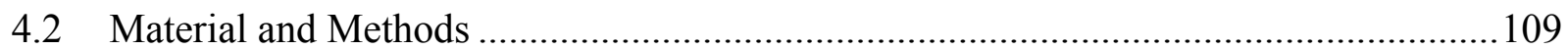




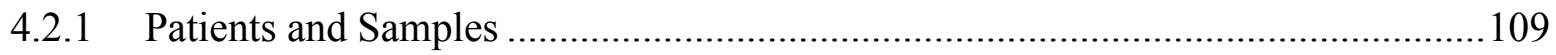

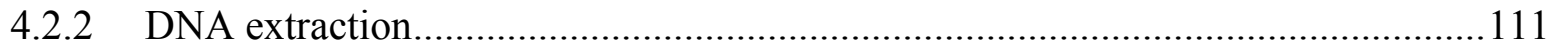

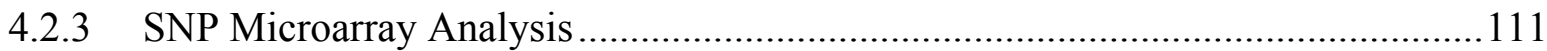

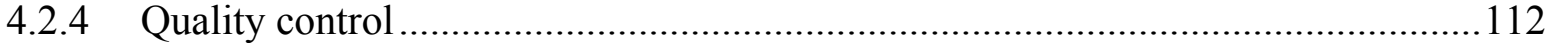

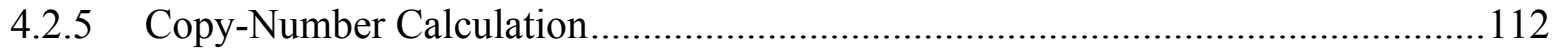

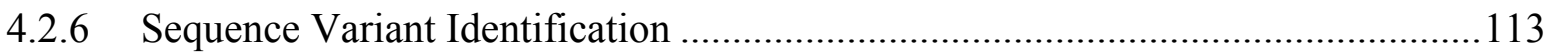

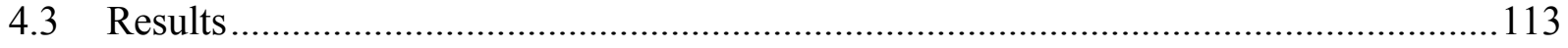

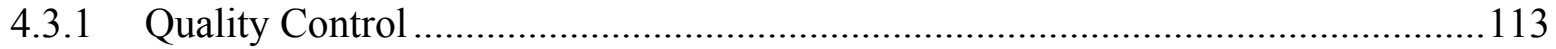

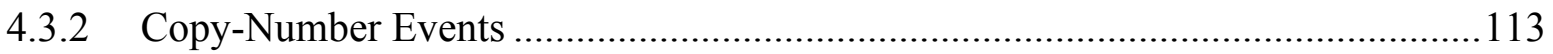

4.3.3 Sequence Variation Events ............................................................................117

4.4 Discussion and Conclusions …………………......................................................117

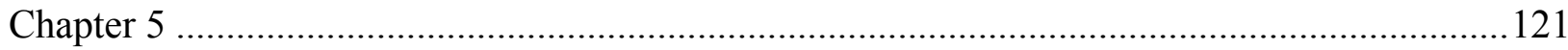

5. General Discussion, Future Directions, and Conclusions ………………………...............122

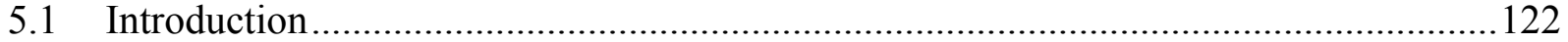

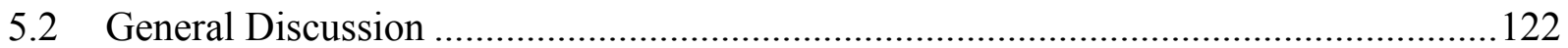

5.2.1 The role of microRNA-375 and metadherin in NPC .........................................122

5.2.1.1 Scientific impact and subsequent research .....................................................123

5.2.2 MiRNA Signature of Distant Metastasis in NPC ................................................124

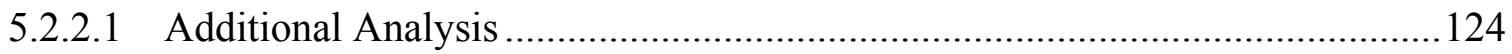

5.2.3 Genomic Characterization of NPC Samples ....................................................127

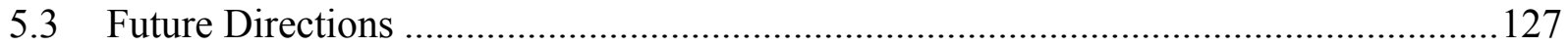

5.3.1 The role of microRNA-375 and metadherin in NPC ..........................................127

5.3.1.1 Clinical Implementation ...........................................................................127

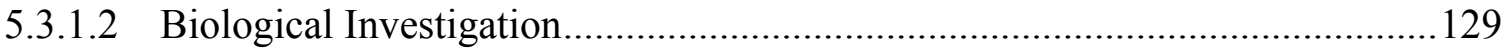

5.3.2 MiRNA Signature of Distant Metastasis in NPC ...................................................130

5.3.2.1 Clinical Implementation ..........................................................................130

5.3.2.2 Biological Investigation..............................................................................131

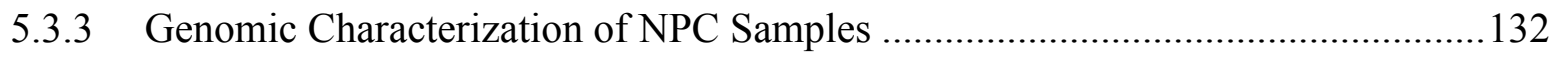

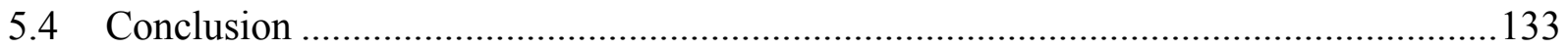

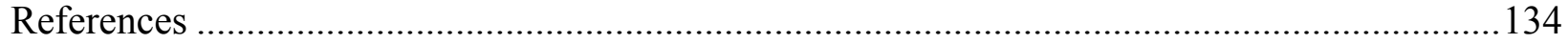




\section{List of Tables}

Table 1-1. Summary of miRNA expression in studies using primary NPC samples. ................22

Table 1-2. Comparison of different miRNA profiling platforms and technologies ....................31

Table 2-1. Clinical characteristics of HNC patients studied ...............................................40

Table 2-2. Primer sequences for qRT-PCR of potential miR-375 targets. ..............................45

Table 2-3. Primer sequences used for cloning and screening of pmiR-REPORT luciferase encoding vectors.

Table 2-4. Potential miR-375 targets identified using both DNA micro-arrays and in silico

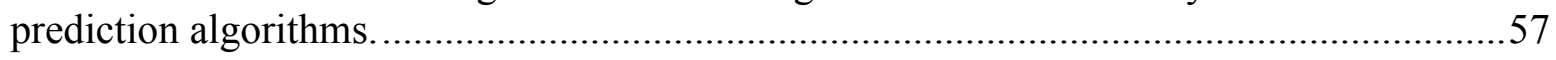

Table 3-1. Clinical characteristics of patients in the two independent cohorts studied. .81

Table 3-2. Differential expression analysis statistics for 81 miRNAs found to be significantly altered in both Training and Validation cohorts.

Table 3-3. MiRNAs with non-zero coefficients in the penalized regression model fitted to miRNA expression data from the training set.

Table 3-4. Univariate and multivariate CoxPH analysis of clinical factors and miRNA-signature Risk-Score in the combined dataset from both the Training and Validation cohorts $(n=242)$.

Table 3-5. C-statistics for comparison of various CoxPH models' ability to predict DMFS......97

Table 3-6. Pathways observed to be significantly enriched within validated targets of the four miRNAs in the prognostic signature using DAVID (v6.7). 100

Table 4-1. Clinical characteristics of 15 NPC patients analyzed using OncoScan platform.....110

Table 4-2. Genomic regions with significant $(\mathrm{p}<0.05) \mathrm{CNV}$ events. 115 


\section{List of Figures}

Figure 1-1. Anatomical structures from which head and neck cancers can arise.

Figure 1-2. Summary of the host-EBV relationship promoting oncogenic phenotypes in NPC cells.

Figure 1-3. miRNA Biogenesis.

Figure 2-1. Genomic location of miR-375 at chromosome $2 \mathrm{q} 35$.

Figure 2-2. miR-375 expression in HNC cell lines. .53

Figure 2-3. Down-regulation of miR-375 in HNC cells .54

Figure 2-4. Prognostic significance of miR-375 expression in NPC patient samples.................55

Figure 2-5. Expression of putative targets in HNC cells after miR-375 transfection. .58

Figure 2-6. Schematic depicting the location of predicted miR-375 binding sites on the MTDH transcript.

Figure 2-7. Identification of mRNA targets of miR-375.

Figure 2-8. Interaction of miR-375 with MTPN and USP1 3' UTRs.

Figure 2-9. Specificity of siRNA mediated cytotoxic effects. 64

Figure 2-10. Migration and invasion of miR-375 or siMTDH in HNC cells.

Figure 2-11. Down-stream signaling, and in vivo effects of miR-375 and siMTDH in HNC models.

Figure 2-12. MTDH Expression in HNC patient samples. .70

Figure 2-13. Association of MTDH expression with treatment modality and tumour stage ......71

Figure 2-14. Working model detailing the role of the miR-375-MTDH axis in NPC .76

Figure 3-1. Kaplan-Meier curves showing NPC patients dichotomized based on risk score.......91

Figure 3-2. Kaplan-Meier curves demonstrating the association Disease Specific Survival the 4-miRNA signature applied to HNSCC miRNA-Seq Data generated by TCGA

Figure 3-3. Kaplan-Meier curves demonstrating cumulative incidence of distant metastasis based on clinical Nodal-stage in the combined dataset $(n=245)$. 
Figure 3-4. Comparison of 4-miRNA signature with clinical variables.

Figure 3-5. Kaplan-Meier curves showing DM in NPC patients dichotomized based on miRNA risk score in advanced stage patients (Stage III/IV).

Figure 3-6. Kaplan-Meier curves showing local-regional relapse (LRR) in NPC patients dichotomized based on miRNA RS in advanced stage patients (Stage III/IV).

Figure 3-7. Comparisons of the current 4-miRNA signature vs. the 5-miRNA signature reported by Liu et al. ${ }^{34}$

Figure 4-1. Visual representation of genome-wide frequency of CNVs across 15 NPC samples.

Figure 4-2. Representative sample showing a small (318kb) amplification at Chr18q21.33....120

Figure 5-1. Comparison of the prognostic ability of MTDH, the 4-miRNA signature and $\mathrm{N}$-stage; combined and individually 


\section{List of Abbreviations}

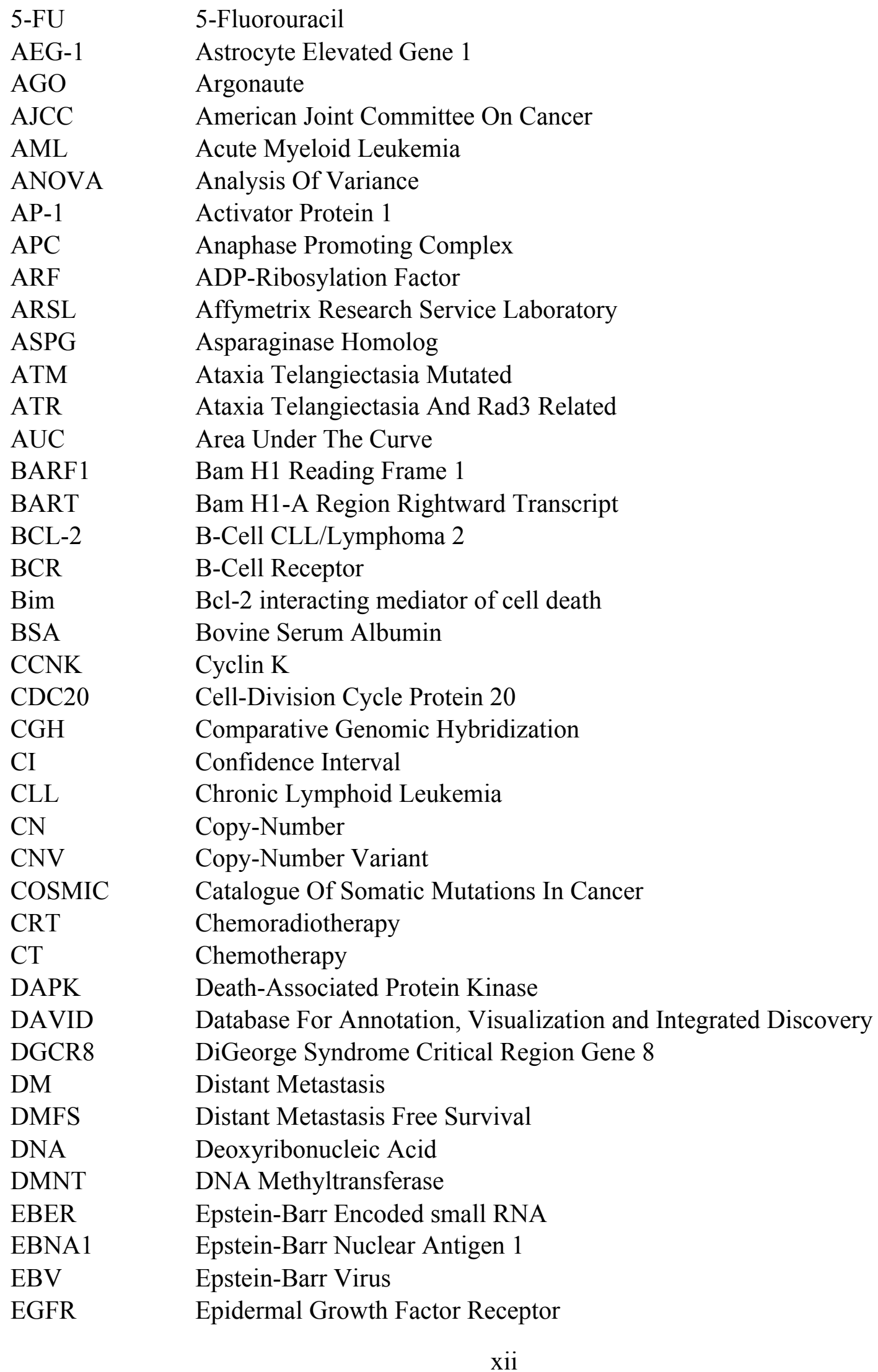




\begin{tabular}{ll} 
EMT & Epithelial To Mesenchymal Transition \\
EVP & Events Per Variable \\
FACS & Fluorescence-Activated Cell Sorting \\
FAS & Fas Cell Surface Death Receptor \\
FC & Fold Change \\
FDR & False Discovery Rate \\
FFPE & Formalin Fixed Paraffin Embedded \\
FLT3 & Fms-Related Tyrosine Kinase 3 \\
FMRP & Fragile X Mental Retardation 1 Protein \\
GAPDH & Glyceraldehyde-3-Phosphate Dehydrogenase \\
GHSR & Growth Hormone Secretagogue Receptor \\
GMFB & Glia Maturation Factor, Beta \\
GTP & Guanosine Triphosphate \\
HCC & Hepatocellular Carcinoma \\
HDAC & Histone Deacetylase \\
HIF1 & Hypoxia-Inducible Factor 1A \\
HMM & Hidden Markov Model \\
HNC & Head And Neck Cancer \\
HNSCC & Head And Neck Squamous Cell Carcinoma \\
HR & Hazard Ratio \\
HT & High-Throughput \\
HTS & High-Throughput Sequencing \\
IFN & Interferon \\
IGF1 & Insulin-like Growth Factor 1 \\
IHC & Immunohistochemistry \\
IMRT & Intensity Modulated Radiation Therapy \\
INF & Interferon \\
IPO7 & Importin 7 \\
KEGG & Kyoto Encyclopedia Of Genes And Genomes \\
KM & Kaplan-Meier \\
KRAS & Kirsten Rat Sarcoma Viral Oncogene Homolog \\
LCM & Laser-Capture Microdissected \\
LMP & Latent Membrane Protein \\
LNA & Locked-Nucleic Acid \\
LOH & Loss of Heterozygosity \\
LRC & Locoregional Control \\
LRR & Locoregional Relapse \\
LTBR & Lymphotoxin-Beta Receptor \\
MAF & Minor Allele Frequency \\
MAPD & Median Absolute Pairwise Difference \\
MAPK & Mitogen-Activated Protein Kinases \\
MHC & Major Histocompatibility Complex \\
MICB & MHC class I-related chain B \\
& \\
\hline
\end{tabular}




$\begin{array}{ll}\text { miRNA } & \text { MicroRNA } \\ \text { MIP } & \text { Molecular Inversion Probe } \\ \text { miRISC } & \text { MiRNA Inducible Silencing Complex } \\ \text { MMP } & \text { Matrix Metalloproteinases } \\ \text { mRNA } & \text { Messenger RNA } \\ \text { MTDH } & \text { Metadherin } \\ \text { MTHFSD } & \text { Methenyltetrahydrofolate Synthetase Domain Containing } \\ \text { MTPN } & \text { Myotrophin } \\ \text { NCI } & \text { National Cancer Institute } \\ \text { NF-kB } & \text { Nuclear Factor Kappa-Light-Chain-Enhancer Of Activated B Cells } \\ \text { NOE } & \text { Normal Oral Epithelium } \\ \text { NOX2 } & \text { NADPH Oxidase 2 } \\ \text { NP } & \text { Nasopharynx } \\ \text { NPC } & \text { Nasopharyngeal Carcinoma } \\ \text { NuA } & \text { Nucleic Acid } \\ \text { OD } & \text { Optical Density } \\ \text { OS } & \text { Overall Survival } \\ \text { PAGE } & \text { Polyacrylamide Gel Electrophoresis } \\ \text { PBS } & \text { Phosphate Buffered Saline } \\ \text { PCR } & \text { Polymerase Chain Reaction } \\ \text { PET } & \text { Polyethylene Terephthalate } \\ \text { PI } & \text { Propidium Iodide } \\ \text { PKR } & \text { Protein Kinase RNA-Dependent } \\ \text { PLEK } & \text { Pleckstrin } \\ \text { PM } & \text { Princess Margaret Cancer Center } \\ \text { PNA } & \text { Peptide-Nucleic Acid } \\ \text { Pol } & \text { Polymerase } \\ \text { pre-miRNA } & \text { Precursor microRNA } \\ \text { pri-miRNA } & \text { Primary microRNA } \\ \text { PTEN } & \text { Phosphatase And Tensin Homolog } \\ \text { PUMA } & \text { p53 Up-Regulated Modulator of Apoptosis } \\ \text { QC } & \text { Quality Control } \\ \text { RASSF1A } & \text { Ras Association Domain Family Member 1A } \\ \text { RCT } & \text { Randomized Controlled Trial } \\ \text { REB } & \text { Research Ethics Board } \\ \text { RELA } & \text { V-Rel Avian Reticuloendotheliosis Viral Oncogene Homolog A } \\ \text { RIG1 } & \text { Retinoic Acid-Inducible Gene-Like Receptor 1 } \\ \text { RNA } & \text { Ribonucleic Acid } \\ \text { ROC } & \text { Receiver Operating Characteristic } \\ \text { RPM } & \text { Reads Per Million } \\ \text { RS } & \text { Risk-Score } \\ \text { RT } & \text { Reverse Transcription } \\ \text { SCID } & \text { Severe Combined Immunodeficient } \\ & \\ & \\ & \\ & \end{array}$




$\begin{array}{ll}\text { SE } & \text { Standard Error } \\ \text { siRNA } & \text { Small Interfering RNA } \\ \text { SNP } & \text { Single Nucleotide Polymorphism } \\ \text { SP-1 } & \text { Specificity Protein 1 } \\ \text { STAC } & \text { Significance Testing For Aberrant Copy-Number } \\ \text { STR } & \text { Short Tandem Repeat } \\ \text { TAPBPL } & \text { Tap Binding Protein-Like } \\ \text { TCGA } & \text { The Cancer Genome Atlas } \\ \text { TGF } \beta & \text { Transforming Growth Factor } \beta \\ \text { TLD } & \text { Tumour plus Leg Diameter } \\ \text { TLDA } & \text { Taqman Low Density Array } \\ \text { TLR3 } & \text { Toll-Like Receptor 3 } \\ \text { TMP1 } & \text { T-Cell Lymphoma Invasion And Metastasis 1 } \\ \text { TNF } & \text { Tumour Necrosis Factor A } \\ \text { TNM } & \text { Tumour Node Metastasis } \\ \text { TOMM22 } & \text { Translocase Of Outer Mitochondrial Membrane 22 Homolog } \\ \text { TRADD } & \text { TNFR-Associated Death Domain } \\ \text { TRAF } & \text { TNFR-Associated Factor } \\ \text { TRBP } & \text { Trans-Activation-Responsive RNA-Binding Protein } \\ \text { UTR } & \text { Un-Translated Region } \\ \text { VEGF } & \text { Vascular Endothelial Growth Factor } \\ \text { WHO } & \text { World Health Organization }\end{array}$


Chapter 1

\section{Introduction}




\section{Introduction}

\subsection{Nasopharyngeal Carcinoma}

Nasopharyngeal carcinoma (NPC) is an epithelial malignancy of the uppermost portion of the pharynx, located posterior to the nasal cavity, extending from the upper surface of the soft palate to the base of the skull (Figure 1-1). NPC is characterized by a unique set of geographical, etiological, and biological features that distinguish it from other cancers of the head and neck region. The prevalence of NPC is highest in distinct endemic regions such as Southeast Asia, where the annual incidence can reach up to $1 / 4000^{1}$. In contrast, NPC is uncommon in Canada, although in populous and ethnically diverse cities such as Toronto, up to $\sim 150$ new cases of NPC are diagnosed each year.

The World Health Organization (WHO) defines the following histological subtypes of NPC:

1. Keratinizing squamous cell carcinoma

2. Non-keratinizing carcinoma

A. Differentiated type

B. Undifferentiated type

3. Basaloid squamous cell carcinoma

Subtypes $2 \mathrm{~A} \& \mathrm{~B}$ and are by far the most prevalent, accounting for nearly all NPC cases diagnosed in endemic regions ${ }^{1}$.

\subsubsection{Environmental Risk Factors for NPC}

The most common causal agent in NPC is the Epstein-Barr Virus (EBV). The EBV genome is present in $\sim 90 \%$ of NPCs observed in endemic regions, and is thought to play an 
important role in many aspects of NPC development ${ }^{2}$. How EBV contributes to NPC development will be covered in more depth in section 1.1.3.2.

Other environmental risk factors associated with NPC have also been identified; largely through prospective epidemiologic studies. Like other cancers of the upper aerodigestive tract, risk of NPC is significantly increased by alcohol consumption and tobacco smoking ${ }^{1}$. Notably, a stronger association between tobacco smoking and NPC is observed in populations outside endemic regions than those within these high-risk populations (OR: 1.76 vs. 1.29, respectively) $)^{3}$. Relatedly, NPC patients with differentiated-type disease (the predominant type in non-endemic populations) have a greater association with tobacco smoking than those with undifferentiated type disease (OR: 2.2 vs. 1.27 , respectively) ${ }^{3}$.

A variety of dietary habits are also positively and negatively correlated with NPC. For instance, consumption of salt preserved foods has consistently been confirmed to be associated with an increased risk of $\mathrm{NPC}^{1,4,5}$. The process used to preserve such foods is thought to increase levels of carcinogenic nitrosamines ${ }^{6}$, thus leading to the $\sim 2$-fold increased incidence of NPC in frequent consumers of these preserved foods ${ }^{5}$. Additional environmental risk factors that may have a link to NPC include alcohol drinking, wood-fire smoke, occupational exposures and some herbal medicines ${ }^{1}$.

In contrast, high consumption of fresh fruits and vegetables has been shown to decrease an individual's risk of NPC ${ }^{1,5}$; which could be attributed to many components within fruits and vegetables that may prevent cancer such as fiber, vitamins, folate, and carotenoids ${ }^{7}$. 


\section{Head and Neck Cancer Regions}

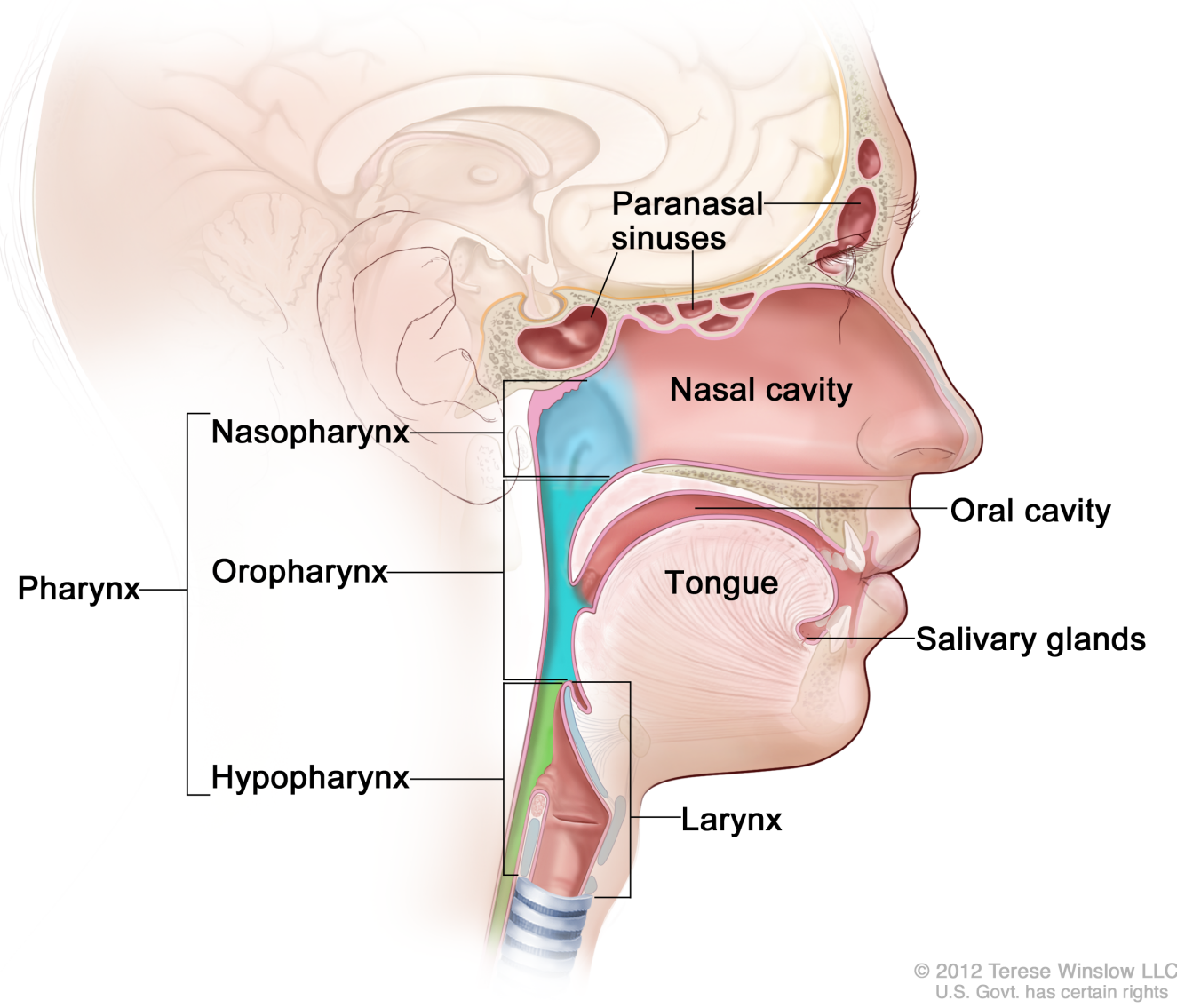

Figure 1-1. Anatomical structures from which head and neck cancers can arise. Image printed with permission from the artist. (C) 2012 Terese Winslow LLC, U.S. Govt. has certain rights. 


\subsubsection{Clinical characteristics of NPC}

NPC patients who present with early stage disease (T1-2aN0M0) are commonly treated with curative radiation therapy (RT) alone $^{8}$. Prognosis is usually excellent in these patients with 5-year local control rates of up to $95 \%{ }^{8}$. The majority of NPC patients, however, present with locally advanced disease and thus have a far worse prognosis. These patients have been shown to benefit significantly from concomitant chemo-radiotherapy (CRT) using Cisplatin or 5-fluorouracil (5-FU) ${ }^{9}$. Indeed, studies comparing CRT vs. RT alone report 3-year overall survival rates of $78-84 \%$ vs. $47-65 \%$, respectively, in favour of the combinatorial strategy ${ }^{10-12}$. Despite the obvious survival benefit, which has been documented in randomized clinical trials (RCTs), CRT also results in a higher incidence of acute and late toxicities compared with RT alone $^{13,14}$. This highlights the importance of selecting patients with advanced disease who could be cured with RT alone, and thereby spared the potential morbidities of combined therapy, as well as the development of novel, less toxic therapies. Moreover, while the implementation of CRT for advanced NPC patients has shown a marked improvement in local control, distant control has not improved substantially, with $\sim 17 \%$ of advanced-stage patients treated with modern modalities still developing distant metastases within 3 years ${ }^{15}$.

\subsubsection{Molecular biology of NPC}

The majority of NPCs arise as a result of EBV infection combined with additional genetic and epigenetic aberrations, which collaboratively promote the proliferation, survival, and motility of the malignant epithelial cells ${ }^{16}$. While EBV is likely an early event in nasopharyngeal tumourigenesis, there is mounting evidence to suggest that pre-existing genetic abnormalities are required to support the persistent infection of pre-malignant and malignant 
epithelial cells of the nasopharynx as well ${ }^{17,18}$. This complex interplay between EBV infection and host alterations is summarized in Figure 1-2.

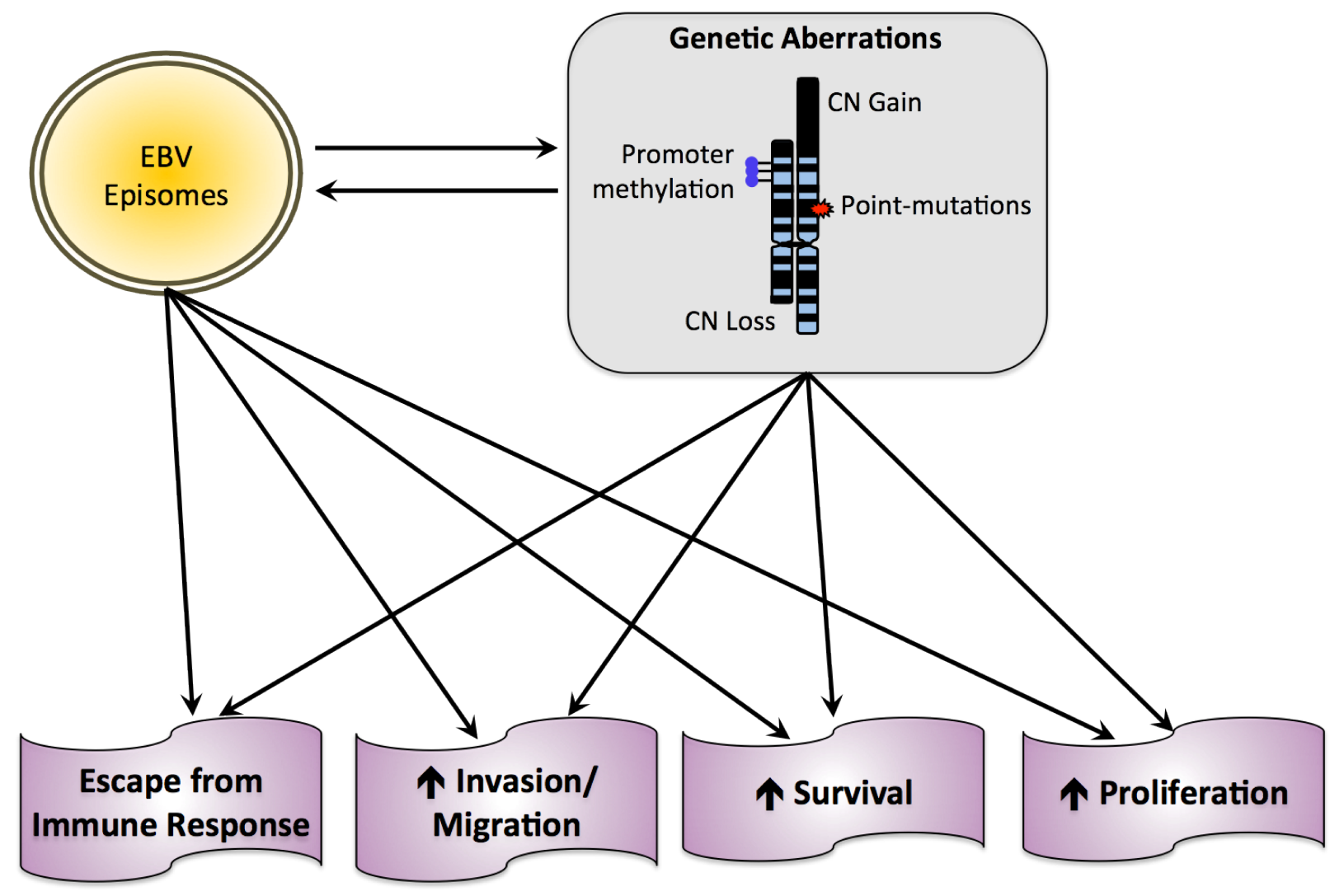

Figure 1-2. Summary of the host-EBV relationship promoting oncogenic phenotypes in NPC cells. 


\subsubsection{Genetic alterations in NPC}

The majority of genomic studies on NPC have focused on gross chromosomal abnormalities utilizing cytogenetic techniques such as G-banding, comparative genomic hybridization $(\mathrm{CGH})$, and array $\mathrm{CGH}(\mathrm{aCGH})$ to detect copy number events and translocations. The first studies examined cell lines and xenografts, identifying multiple aberrations on chromosome 3, including duplications, translocations and deletions ${ }^{19-21}$. Subsequent studies in primary NPC samples led to the identification of a number of frequently altered genomic regions now known to harbour oncogenes and tumour suppressors important in NPC development and progression.

Important examples of recurrent chromosomal events in NPC include focal losses on the $\mathrm{p}$ arm of chromosomes 3 and 9, and the $\mathrm{q}$ arm of chromosomes 11,13,14, and 16. These have been identified and confirmed in multiple studies $^{22-28}$, corresponding to the genetic loci of multiple tumour suppressors critical in NPC. One important region is the $9 \mathrm{p} 21.3$ cytoband, which was originally observed to exhibit copy number losses in a majority (61\%), and a homozygous deletion in one of the primary NPC samples tested ${ }^{28}$. Contained within this region are two genes which encode three tumour suppressor proteins: p16 (INK4a), ARF (gene: CDKN2A) and p15 (INK4b) (gene: CDKN2B). Hypermethylation of the p16 promoter was also frequently detected in NPC samples, indicating that this was the target of copy number loss in this region ${ }^{29,30}$. In subsequent studies, the functional implication of p16 loss in NPC was examined. Wang et al. ${ }^{31}$ demonstrated that re-expression of p16 in NPC cell lines led to G0/G1 arrest along with reduced in vivo tumourigenicity, most likely mediated via its canonical role as an inhibitor of the interaction between cdk4/cdk6 and cyclin D1 (CCND1). Moreover, it was also observed that deletion of p16 was a critical event in hTERT (telomerase) immortalization 
of normal nasopharyngeal epithelial cells ${ }^{32}$, providing support for p16 inactivation as an early event in the development of $\mathrm{NPC}^{31}$.

Similar to p16, Ras Association Domain Family Member 1A (RASSF1A) was identified as an important tumour suppressor in NPC due to the observation of frequent deletion of its genomic locus at $3 \mathrm{p} 21.3$, and methylation of its promoter ${ }^{22,33,34}$. In addition to copy number and methylation aberrations, somatic mutations in RASSF1A have also been detected in a small proportion of NPC samples $(9.5 \%)^{34}$. The RASSF 1 gene was first cloned in 2000 by Dammann et $a{ }^{35}{ }^{35}$, where they reported the expression of three transcripts (RASSF1A/B/C) through alternative splicing and promoter usage. Since its discovery, multiple putative functions have been suggested for RASSF1A, although its precise role remains controversial ${ }^{36}$. Only a few studies have explored the role of RASSF1A in NPC specifically. Chow et al. ${ }^{37}$ examined the tumour suppressive function of RASSF1A by stably expressing the gene in the EBV-positive NPC cell line, C666-1, which has lost expression of RASSF1A through promoter hypermethylation $^{34}$. This study demonstrated that re-expression of $R A S S F 1 A$ resulted in growth inhibition both in vitro and in vivo ${ }^{37}$. This group went on to show that RASSF1A regulated the expression of genes involved in the tumour growth factor $\beta$ (TGF- $\beta$ ) signaling pathway (activin $\beta \mathrm{E}, \mathrm{Id} 2)^{38}$. RASSF1A is also known to play an important role in regulating mitotic progression through direct inhibition of the APC (anaphase promoting complex)-CDC20 (cell-division cycle protein 20) complex, which functions to promote microtubule stability ${ }^{39-41}$. Hence, when RASSF1A expression was suppressed in immortalized nasopharyngeal epithelial cells, microtubule structures were disrupted, and chromosomal instability ensued, thereby enhancing tumourigenicity ${ }^{42}$. 
A number of oncogenes are also activated in NPC via genetic and epigenetic mechanisms. Frequent amplification of 11q13 was found in multiple cytogenetic studies, with CCND1 identified as the likely target of these copy number gains ${ }^{22,43}$. Moreover, CCND1 was observed to be over-expressed in $>90 \%$ of primary NPC samples by immunohistochemical (IHC) staining, and its knockdown in NPC cell lines resulted in a significant decrease in cellular proliferation $^{43}$. Similarly, recurrent amplification of 12 p13 was found to target lymphotoxinbeta receptor (LTBR), overexpression of which was confirmed to contribute to cell survival and tumour growth in NPC models via activation of the NF- $\kappa$ B pathway ${ }^{44}$. Likewise, an increase in copy number of PIK3CA at 3q26.1 has been observed in $70-75 \%$ of primary NPC samples ${ }^{26,45}$, and IHC evidence indicated that the PI3K-Akt pathway was activated in $\sim 85 \%$ of NPC samples $^{46}$. Hence, given that PIK3CA point mutations are only rarely detected in NPC, amplification is likely the major mechanism of constitutive activation of this pathway ${ }^{45}$.

While no comprehensive sequencing studies have yet been reported in NPC, a small number of point mutations have been identified through targeted sequencing of specific tumour suppressor and oncogenes. TP53, the most widely mutated gene in human cancers, is rarely mutated in NPC $(0-8 \%)^{47-49}$. Although the major mechanisms of RASSF1A deactivation are copy number loss and promoter hypermethylation, mutations have been detected in $\sim 10 \%$ of NPC samples ${ }^{34}$. Finally, infrequent mutations in PIK3CA, and three NF- $\kappa$ B pathway regulators (TRAF2, TRAF3, and A20) have been reported; all in less than $10 \%$ of samples examined $^{50,51}$. The small number of identified point mutations in NPC is likely due in part to a lack of highthroughput sequencing studies in this disease. It is also probable that because EBV infection functions to activate and suppress many of the necessary signaling pathways in NPC cells, somatic mutations are of less importance than in other, non-virally driven cancers. 


\subsubsection{The role of EBV in NP tumourigenesis}

EBV is an enveloped, double stranded DNA, human herpesvirus that is associated with a number of human malignancies, including Burkitt's lymphoma, Hodgkin's lymphoma, gastric carcinoma and NPC. NPC cells exhibit a type II EBV latency profile, resulting in the expression of several protein-coding (Epstein-Barr nuclear antigen 1 (EBNA1), Bam H1 Reading Frame 1 (BARF1), latent membrane proteins (LMPs) 1, 2A, and 2B)), and non-protein coding (Epstein-Barr encoded small RNAs (EBERs) 1 and 2, Bam H1-A region rightward transcript (BART) miRNAs) EBV genes with a variety of oncogenic properties ${ }^{46,52-54}$.

\subsection{LMP1}

Perhaps the most well characterized EBV encoded protein in the context of NPC is LMP1. LMP1 is thought to act, at least in part, as a viral mimic of the TNF- $\alpha$ receptor family members TNFR1 and CD4055. Although LMP1 shares little sequence homolog to these receptors, it is capable of modulating a number of TNF- responsive signaling proteins including TNFR-associated factors (TRAFs) and the TNFR-associated death domain protein (TRADD) ${ }^{55,56}$. Unlike TNFRs however, LMP1 does not depend on ligand activation, but is constitutively activated and consequently promotes tumour development and progression via a number of signaling pathways ${ }^{57,58}$.

Early studies showed that expression of LMP1 transformed immortalized rodent cells, and inhibited differentiation in human epithelial cells ${ }^{59,60}$. Subsequent studies provided evidence that LMP1 is capable of promoting all of the "hallmarks of cancer" LMP1 has been shown to increase NPC cell proliferation and survival by inducing the expression of mitogenic receptors such as EGFR ${ }^{62,63}$ and c-Met ${ }^{64}$, as well as activating the progrowth and anti-apoptotic NF- $\mathrm{B}^{65}, \mathrm{PI} 3 \mathrm{~K} / \mathrm{Akt}^{66}$, and MAPK kinase pathways ${ }^{67}$. LMP1 has also 
been found to increase the metastatic potential of NPC cells through induction of epithelial to mesenchymal transition (EMT) ${ }^{68,69}$, as well as increased expression and the release of matrix metalloproteinases (MMPs) ${ }^{70,71}$ through activation of NF-kB, SP-1 (specificity protein 1), AP-1 (activator protein 1), and ERK-MAPK pathways ${ }^{70,72-74}$. Evidence also indicates that LMP1 promotes angiogenesis in the NPC microenvironment through the induction of HypoxiaInducible Factor $1 \alpha$ (HIF-1 $\alpha$ ), and Vascular Endothelial Growth Factor (VEGF) ${ }^{75-78}$. Notably, high LMP1 expression in primary NPC samples has also been shown to be associated with regional and distant metastasis ${ }^{79}$. Another mechanism of action of LMP1 is modulation of epigenetic marks on the host genome ${ }^{58}$. Specifically, LMP1 has been demonstrated to induce the expression and activity of DNA methyltransferases (DMNTs) 1, 3a, and 3b, resulting in promoter hypermethylation of the tumour suppressor gene E-cadherin, and possibly others ${ }^{68,80}$.

Despite its many functions in NPC development and progression, LMP1 expression is heterogeneous within and amongst NP tumours ${ }^{81}$. Indeed, LMP1 expression is undetectable by current techniques in as much as $50 \%$ of NPC specimens examined ${ }^{79}$. As such, additional EBV encoded genes also function in concert with LMP1 to promote NP oncogenesis through both overlapping and novel mechanisms.

\subsection{LMP2A/B}

In contrast with LMP1, LMP2A is consistently expressed in up to $100 \%$ of NPC tumours while LMP2B expression mirrors LMP1 more closely with expression observed in $\sim 40 \%$ of NPC biopsies ${ }^{81}$. While LMP2A and B do not appear to transform normal cells alone, they do function to regulate several cellular pathways resulting in a variety of oncogenic changes in NPC cells ${ }^{82}$. 
LMP2A transfected epithelial cells exhibit increased proliferation, EMT, invasion and migration through the activation of a variety of signaling pathways including Notch, ERK, Syk, PI3K/Akt, and Wnt/beta-catenin ${ }^{83-90}$. LMP2A has also been demonstrated to negatively regulate the B-cell receptor (BCR) in epithelial cells, resulting in inhibition of a number of $\mathrm{BCR}$ regulated processes including intracellular calcium release, phospho-tyrosine signaling, and the switch from latency to lytic replication ${ }^{91}$. Although slightly less well understood, LMP2B has also been found to activate PI3K/Akt signaling, and can promote cell motility through disruption of cellular adhesion ${ }^{86}$. Finally, both LMP2A and B have been shown to impart resistance to anti-viral IFN signaling in NPC cells through the increased turnover and degradation of INF responsive receptors, IFNAR and IFNGR ${ }^{92}$.

\subsection{EBNA1}

EBNA1 is the only EBV encoded protein expressed in all EBV-associated tumours, and its expression is both necessary and sufficient for the stable maintenance of EBV episomes in dividing cells through its interactions with EBV oriP ${ }^{93,94}$. EBNA1 is also capable of inducing the expression of other EBV encoded genes through transcriptional activation of the $\mathrm{Cp}$ and LMP promoters ${ }^{95,96}$. In addition, evidence suggests that EBNA1 directly promotes oncogenesis in NPC cells, aside from its role in the maintenance of the EBV genome and modulation of other EBV genes ${ }^{54}$. Indeed, knockdown of EBNA1 via RNA interference resulted in reduced cell proliferation and survival in an NPC derived cell line ${ }^{97}$, and its ectopic expression promoted tumour growth and metastasis in NPC xenografts ${ }^{98}$.

It has been demonstrated that a variety of EBNA1-host interactions are at play in NPC cells resulting in modulation of numerous cellular pathways and alteration of cellular homeostasis $^{54}$. Interestingly, EBNA1 has been shown to induce reactive oxygen species (ROS) 
in NPC cells, possibly through induction of expression of the ROS producing NADPH oxidase $2(\mathrm{NOX} 2)^{99,100}$. This increase in oxidative stress was also demonstrated to lead to increased DNA damage in B-cells ${ }^{99}$, which if extended to NPC cells could presumably result in additional oncogenic mutations. EBNA1 has also been found to directly modulate the activity of a number of signaling pathways including TGF- $\beta$, STAT1, and NF-kB, leading to a variety of phenotypic outcomes including increased growth, survival, metastasis and angiogenesis ${ }^{100-104}$.

EBNA1 is capable of reducing p53 levels by interacting with the ubiquitin-specific protease, USP7, to prevent the deubiquitination and consequent stabilization of $\mathrm{p} 53^{105,106}$. This relationship was subsequently shown to result in decreased p53 accumulation in response to DNA damage, with a resultant reduction in apoptosis and increased radioresistance in vitro ${ }^{106-}$ 108. Relatedly, EBNA1 has been shown in NPC and gastric carcinoma cell lines to disrupt Promyelocytic leukemia (PML) nuclear bodies ${ }^{107,109}$, which are also involved in p53 activation, apoptosis and DNA repair ${ }^{110-112}$. Moreover, PML loss has also been demonstrated in vivo, wherein EBV-positive gastric carcinoma biopsies were found to have significantly decreased PML as compared to EBV-negative gastric carcinomas ${ }^{109}$.

Finally, a series of Chromatic immune-precipitation (ChIP) experiments have also identified a number of putative EBNA1-binding sites on cellular DNA ${ }^{113-115}$. However, whether EBNA1 functions to directly bind and promote transcription at these sites remains to be shown $^{113,115}$.

\subsection{EBERs}

EBER 1 and 2 are small (167nt, and 172nt long, respectively), highly expressed in NPC cells, with up to 1 million copies detected per cell ${ }^{116-118}$. Evidence suggests that EBERs can function within NPC cells to promote growth, survival and modulation of host-immunity 
through a variety of mechanisms ${ }^{116}$. Studies in the EBV-positive NPC cell line, C666-1, revealed that EBERs can promote cell growth through an autocrine mechanism by inducing the expression and release of insulin like growth factor-1 (IGF-1); possibly through activation of the IGF-1 promoter $^{119}$. Moreover, IGF-1 expression was frequently detected in NPC biopsies, indicating that the EBER/IGF-1 axis may contribute to the development of NPC in vivo as well ${ }^{119}$. In addition, preliminary data suggests that this induction of IGF-1 occurs through the interaction of the EBERs with the double-stranded RNA (dsRNA) sensing Toll-like receptor 3 $(\text { TLR3 })^{120-122}$. EBV also encodes a number of miRNAs (25 precursors, 44 mature) whose expression and function will be explored in section 1.2.6.

\subsubsection{Prognostic/diagnostic biomarkers in NPC}

While there is no ubiquitously utilized clinical biomarker for NPC, a number of diagnostic and prognostic molecular markers have been reported, and validated to varying degrees. The two most well established biomarkers in NPC are circulating antibodies against EBV (serology), and circulating EBV DNA. Indeed, both have been found to be significantly elevated in NPC patients vs. healthy controls in numerous studies ${ }^{123-128}$. While a role as an early diagnostic screening technique has been suggested for EBV serology ${ }^{129-132}$, the utility of circulating EBV DNA for early detection remains unclear ${ }^{133}$. Conversely, while EBV serology appears to lack a relationship with post-treatment tumour burden ${ }^{134,135}$, post-treatment EBV DNA load effectively identifies recurrent or residual disease ${ }^{136,137}$. This feature has been implemented clinically in a recent feasibility study where serial circulating EBV DNA measurement showed promising results as a screen for early detection of distant failure ${ }^{138}$. A number of groups have also shown a role for pre-treatment concentration and post-treatment 
clearance rate of circulating EBV DNA as prognostic biomarkers capable of predicting relapse and survival ${ }^{139-141}$. While pre-treatment concentration of EBV DNA is likely a reflection of initial tumour burden, post-treatment clearance might well provide additional information regarding residual disease and occult metastases ${ }^{136,139,140}$. Notably, Le et al. ${ }^{142}$ and the WHO (NIBSC code: 09/260) have recently proposed strategies to standardize the analytical methodologies used to measure plasma EBV DNA from NPC patients. Standard operating procedures such as these will enable the robust implementation plasma EBV DNA within future randomized trials; which often accrue patients from multiple institutions and even continents ${ }^{142}$.

In addition to EBV DNA and anti-EBV antibodies, several EBV proteins themselves, as well as EBV encoded RNAs have been identified in circulation for NPC patients. Preliminary results indicated that EBV proteins LMP1 and BARF1 could be detected in the plasma of NPC patients; not in healthy controls ${ }^{143}$. In addition, two classes of non-coding RNA molecules have been detected by several groups in the plasma and/or serum of NPC patients: 1) EBV-encoded small RNAs (EBER1/EBER2) ${ }^{144}$, and 2) several EBV encoded miRNAs from the BART genomic region $^{145-147}$. However, the clinical implications of measuring EBV RNA molecules in circulation of NPC patients remain to be determined ${ }^{148}$.

A number of human nucleic acid, protein and metabolite biomarkers have also been proposed for diagnostic and prognostic use in NPC patients as well. These include DNA fragments containing hypermethylated promoter regions for several known tumour suppressor genes (e.g. $C D H 1, D A P K$, and $C D K N 2 A)^{149,150}$, hemoglobin and lactate dehydrogenase ${ }^{151}$, as well as multiple circulating cytokines including TGF- $\beta^{152}$, interferon- $\gamma^{153}$, and CD40-ligand ${ }^{154}$. Moreover, the cytokines MIP- $3 \alpha^{155}$, and CD $23^{156}$ have also been shown to have prognostic potential. 
Finally, a number of molecular entities within the tumour and surrounding stroma themselves have been explored as potential indicators of clinical outcome. Some examples of cellular proteins observed to have putative prognostic ability include caspase-3, nuclear survivin $^{157}$ as well as c-met ${ }^{158}$. However, much like their circulating counterparts, none of these cellular biomarkers have been widely implemented in the clinic.

\subsection{MicroRNAs}

MicroRNAs (miRNAs) are endogenous small (18-25nt) non-protein coding RNA molecules $^{159.7}$. Originally discovered in C. Elegans but have since then been identified in over 200 different species (miRBase, Release 20). MiRNAs primarily function to decrease the expression of protein coding genes by binding their transcripts in a sequence specific manner, however miRNA-target interactions resulting in increased protein expression have also been noted $^{160,161}$. Since their discovery, miRNAs have risen to the status of arguably one of the most important genetic regulators in the mammalian system ${ }^{159}$. Moreover, numerous miRNAs have been reported to function as both oncogenes and tumour suppressors, regulating tumour initiation and progression at all levels ${ }^{162}$.

\subsubsection{MiRNA Biogenesis and Function}

MiRNA biogenesis is a multistep process, which is tightly regulated within the cell. Figure 1-3 depicts the steps in miRNA processing from transcription in the nucleus, to their interaction with mRNAs in the cytoplasm. MiRNA genes can be located in intergenic regions, or within the exons or introns of other genes. Transcription of these miR-genes is performed predominantly by RNA polymerase II (Pol II), and Pol III in some cases ${ }^{163,164}$. A variety of Pol 
II-associated transcription factors have been found to direct miRNA transcription, providing regulation of miRNA expression at the level of transcription ${ }^{165}$. The RNA product that is transcribed is called a primary-miRNA (or pri-miRNA). These pri-miRNAs vary in length from $\sim 100$ to 1000 s of base pairs, and exist in diverse stem-loop structures ${ }^{163}$. Following transcription, the pri-miRNA is cleaved within the nucleus by the type III RNase Drosha, in association with DGCR8 (DiGeorge syndrome critical region gene 8) at the stem of the loop structure to release a $\sim 70$ nt precursor miRNA (pre-miRNA) ${ }^{163}$. This pre-miRNA is then exported out of the nucleus by the dsRNA specific transporting complex composed of Exportin5 and RAN-GTP ${ }^{166}$.

In the cytoplasm, the pre-miRNA is further processed by Dicer and its cofactor Transactivation-Responsive RNA-binding protein (TRBP) to release the $\sim 22 \mathrm{nt}$ mature miRNA duplex $^{163}$. One strand of this duplex, termed the 'guide strand', is then preferentially incorporated into the miRNA inducible silencing complex (miRISC) ${ }^{163}$. Although this process is not completely understood, the guide strand is almost always the strand with the thermodynamically least stable $5^{\prime}$ terminus ${ }^{167}$. The miRISC is a multipart entity with several potential members. The key functional element of the miRISC is the Argonaute protein (in mammals, one of AGO1-4) ${ }^{163}$. In addition to Argonaute, several other proteins may be involved in the miRISC such as the P-body marker, FMRP (Fragile X mental retardation 1 protein) ${ }^{168}$, and the de-capping activator RCK/p54 $4^{169,170}$.

Once a mature miRNA is incorporated, the miRISC can then bind to a target mRNA at a sequence specific binding site. A particular miRNA could bind to hundreds of different genes; sometimes even onto multiple sites for a given target mRNA. MiRNA binding could occur with either perfect complementarity; or more often, with imperfect complementarity ${ }^{162}$. In 
these instances of imperfect complementarity, there is often a short, $\sim 6-8 \mathrm{nt}$ "seed" region, located near the $5^{\prime}$ end of the miRNA, that appears to be of paramount importance in terms of dictating binding to specific target mRNAs ${ }^{159}$. These seed regions are often conserved among species, and form the basis for many of the current in silico target prediction algorithms ${ }^{163,171-}$ 173

MiRNAs can regulate expression of their targets either through mRNA degradation or translational inhibition. In instances of perfect or near-perfect miRNA-mRNA complementarity, degradation of target mRNAs can be mediated by AGO2; the only Argonaute protein which possesses "slicer" activity ${ }^{163}$. In cases of imperfect complementarity, all 4 Argonaute proteins are capable of inhibiting protein translation as part of the miRISC ${ }^{163}$. In addition, miRNA-target relationships with imperfect binding can also result in mRNA degradation through a non-sequence specific mechanism within cytoplasmic processing $(\mathrm{P})$ bodies $^{174}$. In most cases, the net effect of miRISC binding to a target mRNA is a decrease in its protein levels; however recent reports have demonstrated a few instances wherein protein expression is actually increased ${ }^{163}$. For example, miR-10A can increase the expression of several ribosomal proteins through binding to the 5' UTR (untranslated region) of their mRNA transcripts $^{160}$. 


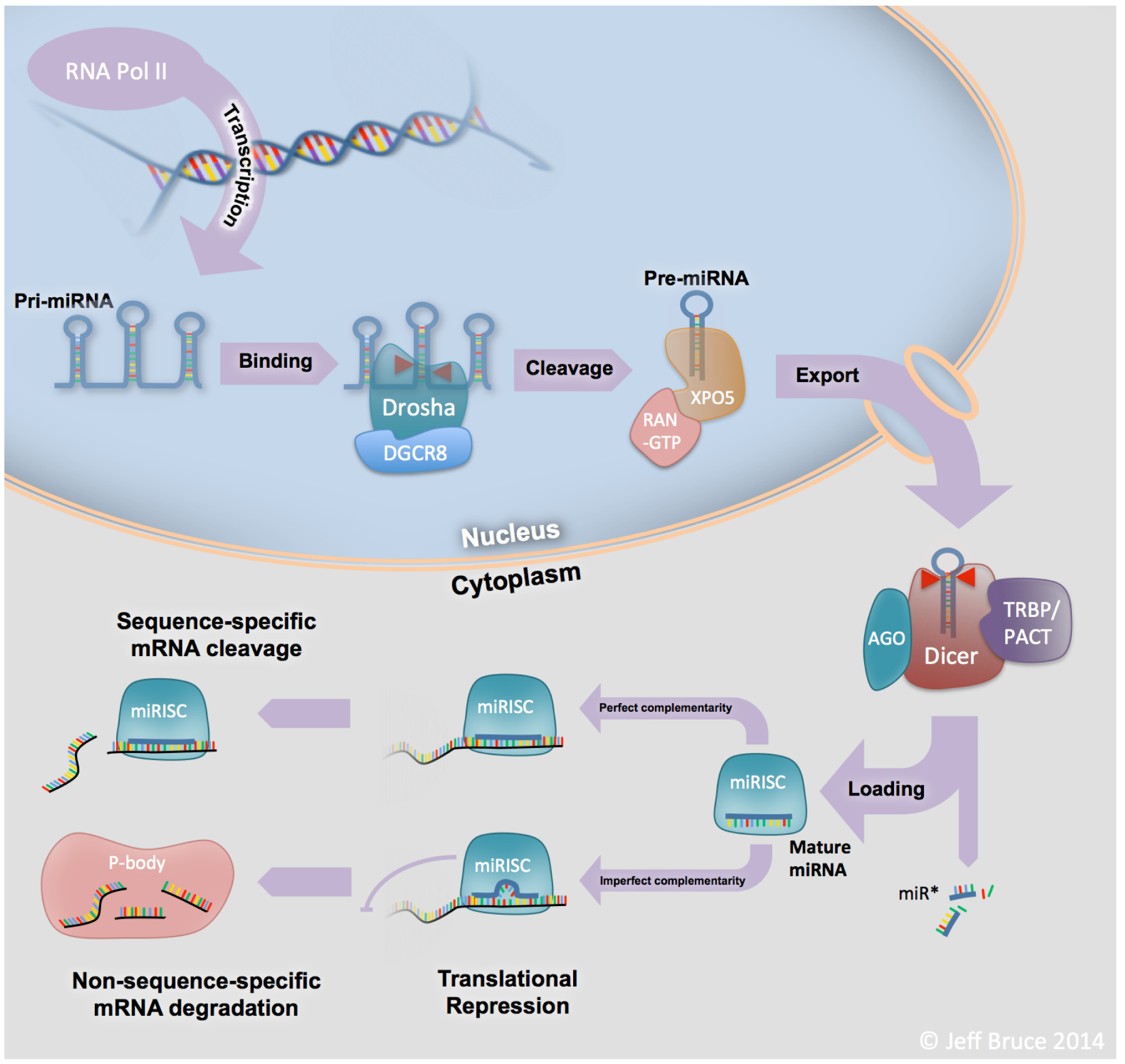

Figure 1-3. miRNA Biogenesis.

See text of section 1.2.1 for full description. RNA Pol II; RNA Polymerase type II, DGCR8; DroshaDiGeorge syndrome critical region gene 8, XPO5; nuclear export factor exportin 5, RAN; Ras-related nuclear protein, GTP; guanosine tri-phosphate, AGO; Argonaute, TRBP; TAR (HIV-1) RNA binding protein 2, PACT; protein kinase, interferon-inducible double stranded RNA. Dicer; Dicer 1, Ribonuclease Type III, miR*; passenger strand from mature miRNA duplex. miRISC; microRNA RNA-induced silencing complex, P-body; processing body. 


\subsubsection{MicroRNAs in Cancer}

As the list of miRNAs has grown, so has our knowledge regarding their biological functions. Indeed, miRNAs have been found to play a role in most, if not all, cellular processes including many pathways related to cancer development and progression. MiRNAs are known to function as tumour suppressor genes or oncogenes, with some miRNAs mediating contradictory roles in different diseases ${ }^{162}$. Aberrant miRNA expression and function has been described in a wide variety of human malignancies, with chromosomal amplifications/deletions, point mutations, or epigenetic alterations as potential causes ${ }^{162}$.

\subsubsection{MicroRNA Expression Signatures in Cancer}

Comprehensive miRNA expression profiling has been performed in a variety of human cancers, yielding a number of interesting observations. Expression signatures have been described which are capable of distinguishing cancerous from normal cells ${ }^{162}$, one cancer from another ${ }^{175}$, predicting response to a particular drug ${ }^{176}$, or predicting patient outcome ${ }^{177,178}$. Indeed, miRNA signatures capable of predicting patient outcome have been developed for a number of human cancers including, but not limited to $\operatorname{lung}^{179,180}$, breast ${ }^{181}$, brain $^{182}$, and chronic lymphocytic leukaemia ${ }^{183}$. While only our 4-miRNA prognostic signature, described in detail in Chapter 3, and one other 5-miRNA signature ${ }^{184}$ exist for NPC, several studies have already reported alterations in expression, and potential biological functions of miRNAs in this disease. 


\subsubsection{Human MicroRNA Expression in Nasopharyngeal Carcinoma}

The first study on the global profiling of miRNAs in NPC was published in 2008 by Paul Ahlquist's group at the National Cancer Institute $(\mathrm{NCI})^{185}$. Using a micro-array based approach to profile 31 laser-capture microdissected (LCM) NPCs and 10 normal nasopharyngeal epithelial samples, they discovered several miRNAs to be deregulated in $\mathrm{NPC}^{185}$. In particular, miR-29c was observed to be significantly down-regulated in NPC, and several miR-29c targets involved in extracellular matrix synthesis and function were identified and validated ${ }^{185}$. Subsequently, other groups have identified a number of dysregulated miRNAs in both NP tumour and blood samples from NPC patients; summarized in Table 1-1. 
Table 1-1. Summary of miRNA expression in studies using primary NPC samples.

\section{NPC Patients vs. Healthy Controls}

\begin{tabular}{|c|c|c|c|}
\hline Up-regulated & Down-regulated & Positive & Negative \\
\hline$*_{\operatorname{miR}}-16^{186}$ & let- $7 g^{187}$ & $\operatorname{miR}-18 a^{188}$ & $*_{\operatorname{miR}-9}{ }^{189}$ \\
\hline${ }^{\mathrm{F}} \mathrm{miR}-17^{190}$ & $* \operatorname{miR}-9^{189}$ & ${ }^{\mathrm{F}} \mathrm{miR}-22^{191}$ & $\operatorname{miR}-26 \mathrm{a}^{184}$ \\
\hline $\operatorname{miR}-18 \mathrm{a}^{\mathbf{1 8 8}}$ & $\operatorname{miR}-26 \mathrm{a}^{168,184,187,192}$ & $\operatorname{miR}-93^{184}$ & $\operatorname{miR}-29 c^{184,193}$ \\
\hline${ }^{\mathrm{F}} \mathrm{miR}-20 \mathrm{a}^{190}$ & $\operatorname{miR}-26 b^{192}$ & ${ }^{\mathrm{F}} \mathrm{miR}-572^{191}$ & $\operatorname{miR}-30 \mathrm{e}^{184}$ \\
\hline${ }^{*} \operatorname{miR}-21^{186}$ & $\operatorname{miR}-29 c^{184,185}$ & ${ }^{\mathrm{F}} \mathrm{miR}-638^{191}$ & $\operatorname{miR}-451^{194}$ \\
\hline${ }^{*}$ miR-24 ${ }^{186}$ & ${ }^{\mathrm{F}} \operatorname{miR}-29 \mathrm{c}^{190}$ & & $\operatorname{miR}-98^{192}$ \\
\hline $\operatorname{miR}-93^{184}$ & $\operatorname{miR}-30 \mathrm{e}^{184}$ & & $\operatorname{miR}-142-3 p^{184}$ \\
\hline $\operatorname{miR}-141^{195}$ & $\operatorname{miR}-34 b^{187}$ & & ${ }^{\mathrm{F}} \mathrm{miR}-1234^{191}$ \\
\hline $\operatorname{miR}-144^{196}$ & $\operatorname{miR}-101^{192}$ & & \\
\hline $\operatorname{miR}-146 a^{197,198}$ & $\operatorname{miR}-138^{199}$ & & \\
\hline $\operatorname{miR}-155^{200}$ & $\operatorname{miR}-142-3 p^{184}$ & & \\
\hline$*_{\operatorname{miR}-155^{186,201}}$ & $\operatorname{miR}-200 b^{202}$ & & \\
\hline${ }^{*} \operatorname{miR}-214-3 p^{189,203}$ & $\operatorname{miR}-216 b^{204}$ & & \\
\hline $\operatorname{miR}-214-3 p^{86}$ & $\operatorname{miR}-218^{205}$ & & \\
\hline $\operatorname{miR}-663^{206}$ & ${ }^{\mathrm{F}} \mathrm{miR}-223^{190}$ & & \\
\hline 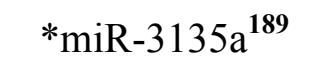 & $*_{\mathrm{miR}-378^{186}}$ & & \\
\hline & $\operatorname{miR}-451^{194}$ & & \\
\hline
\end{tabular}

\section{Prognostic Association}

MiRNAs included in this table satisfy each of the following criteria: 1) a statistically significant alteration in their expression was identified in NPC patient specimens 2) Some degree of validation (either in additional samples, using an additional analytical method, or functional validation) was reported. NPC Patients vs. Healthy Control: expression significantly increased ("up-regulated") or decreased ("down-regulated") in tumours/plasma compared to nasopharyngeal tissues/plasma from healthy controls. Prognostic Association: expression higher ("positive") or lower ("negative") in tumour/plasma samples of patients with poor prognosis. Superscripts: expression measured in *plasma, ${ }^{\mathrm{F}}$ serum, all others were discovered using tumour samples. 


\subsubsection{Human miRNA Biology in NPC}

In addition to alterations in expression of miRNAs in NPC, we and others have also demonstrated phenotypic roles for miRNAs in NP tumourigenesis using in vitro and in vivo models. The first miRNA characterized in the context of NPC was miR-29c, which was the main focus of the first miRNA profiling report by Sengupta et al. ${ }^{185}$. In this initial study, the authors demonstrated a potential tumour suppressive role for miR-29c through targeting mRNAs encoding extracellular matrix proteins (collagens 3A1, 4A1, 15A1, laminin $\gamma 1)^{185}$. They postulated that suppression of miR-29c will subsequently increase the migration and invasion ability of NPC cells through up-regulation of these components of the extracellular matrix; however, they stopped short of testing this hypothesis in the initial study, and further reports supporting these claims have not been published to date. Subsequent studies by two other groups corroborated the role of miR-29c as a tumour suppressor in NPC; however these reports differed on their causal mechanisms, and putative targets. These included promotion of NPC cell migration and invasion through increased expression of T-cell lymphoma invasion and metastasis 1 (TMP1 $)^{207}$, and increased resistance to radiotherapy and Cisplatin through upregulation of anti-apoptotic regulators $\mathrm{Mcl}-1$ and $\mathrm{Bcl}-2^{193}$. This suggests, much like other miRNAs, that miR-29c can act through a number of pathways to suppress proliferation, survival and motility of NPC cells.

Another moderately well studied miRNA in the context of NPC is miR-9. Not only has miR-9 been identified as a potential circulating marker of advanced NPC ${ }^{189}$, several potential targets and functions have also been reported. Recently, two reports on various putative tumour suppressive mechanisms for miR-9 in NPC have been published. Lu et al. described that miR-9 hyper-methylation and subsequent under-expression led to up-regulation of its putative target 
CXCR4, resulting in increased cell growth, migration and invasion through activation of the MAPK pathway ${ }^{208}$. In contrast, Gao et al. ${ }^{209}$ postulated a role for miR-9 in modulating the immune response to NPC by targeting several interferon (IFN)-induced genes, multiple members of the major histocompatibility complex (MHC) class I molecule, as well as a number of interleukins and related genes.

Other potentially functionally active miRNAs in NPC include, but are not limited to, $\operatorname{miR}-26 \mathrm{a}^{210,211}, \operatorname{miR}-98^{192}, \operatorname{miR}-155^{200,212}, \operatorname{miR}-200 \mathrm{a} / \mathrm{b}^{202,213,214}, \operatorname{miR}-205^{215,216}$, and miR$216 \mathrm{~b}^{204}$. Proposed targets of these miRNAs represent a wide array of processes and pathways including $\mathrm{EMT}^{213}, \mathrm{Notch}^{202}$, PI3K-Akt ${ }^{215}$, and ERK/MAPK ${ }^{204}$ signaling pathways. Intriguingly, function, pathways, and targets affected by many of the miRNAs involved in NP tumourigenesis overlap. One such example is miR-26a and miR-218, which have both been found to target Enhancer of Zeste homolog 2 (EZH2), leading to a reduction in multiple tumourigenic properties such as migration, invasion and cell survival ${ }^{192,211}$. This overlapping functional impact of multiple miRNAs adds an increased layer of complexity when attempting to elucidate their biological role; however, this property could also increase our confidence in the biological importance of affected genes and pathways, given that there are multiple mechanisms that malignant cells exploit to activate or inhibit the same gene or pathway.

\subsubsection{EBV encoded miRNAs in Nasopharyngeal Carcinoma}

The EBV, which is present in the vast majority of NPCs, also encodes a number of its own miRNAs. Twenty-five EBV encoded precursor-miRNAs (pre-miRNAs) processed into 44 mature miRNA sequences have been verified to date (miRBase, Release 20). They emanate from two major regions of the EBV genome: a) the BamHI-A rightward transcripts (BART); 
and b) the open reading frame of the $B H R F 1$ gene. Originally cloned from EBV-infected Burkitt's lymphoma cells ${ }^{217}$, BHRF1-miRNAs do not appear to be expressed in EBV-positive NPC primary tissues ${ }^{218,219}$. Indeed, the first report of comprehensive EBV-miRNA profiling in primary NPC samples reported substantial expression of all 35 of the EBV-BART-miRNAs interrogated in the majority of samples, whereas none of the 4 EBV-BHRF1-miRNAs were detected. In contrast, NPC derived cell lines, C666-1 and HONE-Akata, additionally expressed all 4 BHRF1-derived miRNAs ${ }^{218}$. Subsequent studies have identified several targets of EBV miRNAs; both host and viral. Viral targets include LMP1; putatively targeted by several miRNAs from the BART region (ebv-miR-BART1, 9, 16, 17) ${ }^{218,220,221}$, and LMP2A; identified as a target of ebv-miR-BART22 222 . Subsequent functional experiments suggest that BARTmiRNA modulation of these virally encoded proteins can influence multiple cellular properties including cell proliferations, survival, and evasion of host immune response $\mathrm{e}^{220-222}$. Host targets of various BART-miRNAs include pro-apoptotic effectors p53 up-regulated modulator of apoptosis (PUMA) $)^{223}$, Bcl-2 interacting mediator of cell death (Bim) $)^{224}$, and Translocase Of Outer Mitochondrial Membrane 22 Homolog (TOMM22) ${ }^{225}$, as well as several genes thought to influence host immune response, including MHC class I-related chain B (MICB) ${ }^{226}$, importin 7 $(\mathrm{IPO} 7)^{225,227}$, and Dicer ${ }^{228}$. Overall, EBV encoded miRNAs play a complementary role to the viral proteins expressed in NPC; contributing to evasion of the host immune response, and promoting survival and proliferation of NPC cells.

\subsubsection{MicroRNA Expression Profiling Platforms}

The small size and extensive sequence similarity of miRNAs present as unique challenges when attempting to quantitate miRNAs in a biological solution. The "first- 
generation" tools for miRNA expression analysis included northern blotting, quantitative reverse transcription real-time PCR (qRT-PCR), and microarray-based methods. While qRTPCR and microarray-based techniques are still the most widely used methods for miRNA expression analysis, a second generation of counting-based strategies has also emerged. These include the fluorescent-barcode counting method employed by the nCounter platform from Nanostring, and small-RNA sequencing methods, using any of the major next-generation sequencing platforms. Given its limitation in sensitivity and multiplexing compared to other techniques, the use of northern blot analysis for measurement of miRNA expression has waned. Thus, the following review will focus on the other four methodological categories.

\subsubsection{Quantitative RT-PCR}

Two major differences set miRNAs apart from mRNAs pertaining to quantification by qRT-PCR. First, their lack of poly(A) tails means conventional poly-T-primer reverse transcription (RT) utilized for mRNA, can only be applied to miRNA RT with prior addition of poly(A) adapters ${ }^{229}$. Second, conventional PCR primers are generally $\sim 18-22 \mathrm{nt}$ in length to optimize melting and annealing kinetics; thus, given the fact that mature miRNAs are $\sim 22 \mathrm{nt}$ in size themselves, conventional PCR primers are clearly unsuitable for analysis of miRNAs. Two major qRT-PCR chemistries exist, which address both of these issues uniquely.

The Taqman ${ }^{\circledR}$ MicroRNA Assays from Life Technologies ${ }^{\mathrm{TM}}$ (Foster City, CA) first reported by Chen et al. in $2005^{230}$, employ "stem-loop" primers in the RT step to provide sufficient stability of the annealed RNA-primer complex, with complementarity to only 6-9 bases at the 3' end of the miRNA. A forward primer specific to the miRNA of interest, and a generic reverse primer are then employed in the "Real-Time" step, along with a miRNA-unique TaqMan probe, which provides a further level of specificity. These assays have also been 
incorporated into Life Technologies Taqman Low-Density Arrays (TLDA) to allow multiplexing up to 384 primer pairs on a single qPCR plate.

The other major strategy used to improve the binding affinity of primers with short sequences of base-complementarity is the chemical modification of the oligonucleotide backbone. Various such synthetic molecular classes exist; the major categories being peptideand locked-nucleic acids (PNA and LNAs) ${ }^{231}$. The latter class is the intellectual property of Exiqon $^{\mathrm{TM}}$, a company that specializes in techniques for the analysis and manipulation of miRNAs. The C3'-endo sugar conformation of these so-called "locked-nucleic acids" acts to reduce the flexibility of the ribose ring, thereby increasing the affinity of LNA containing oligonucleotides to complementary RNA/DNA sequences ${ }^{232}$. Exiqon's miRCURY LNA ${ }^{\mathrm{TM}}$ Universal microRNA PCR System starts with the ligation of a poly-A tail to mature miRNAs to facilitate universal RT using poly-T primers. This is followed by a qPCR step which employs miRNA specific LNA primers to amplify the sequence of interest, and quantifies the PCR product in real-time using the classic double-stranded DNA dye, SYBR® Green (Life Technologies). Exiqon also offers a line of miRNA qPCR panels which enable the user to multiplex $>750$ miRNA primer sets in a single plate.

\subsubsection{MiRNA Microarrays}

Similar to PCR primers, microarrays rely on base-pairing between complimentary nucleic acid $(\mathrm{NuA})$ sequences, thus raising issues similar to those encountered in PCR pertaining to the small size of miRNAs. As such, similar backbone modification methods to those detailed above, have been exploited to address these concerns for miRNA microarrays as well. 
Microarrays employ oligo-nucleotide probes, which are immobilized on a solid, usually glass surface. Hybridization of labeled sample NuA sequences to complementary probes can then be detected and quantified using a microarray scanner. Exiqon once again offers a line of miRNA microarrays, which capitalize on the binding properties of their LNA chemistry. In addition, the majority of biotechnology companies offering microarrays (e.g. Affymetrix, Agilent, Illumina) also offer miRNA options utilizing their respective methodologies originally developed for mRNA expression profiling. These include variations in fabrication (e.g. printing), labeling (e.g. fluorophore), and experimental design (e.g.. one-colour vs. two-colour) (see review by Heller ${ }^{233}$ ).

\subsubsection{Digital molecular-barcoding expression analysis}

In 2008, Nanostring Technologies launched their first "second-generation" gene expression-profiling platform, nCounter. This platform was subsequently extended in 2010 to include a miRNA expression assay. The nCounter system employs fluorophore-barcodes to label and subsequently "count" specific NuA sequences. The miRNA assay works in much the same way as its mRNA counterpart, with the addition of a ligation step, increasing the length of the miRNAs to improve subsequent hybridization. Much like miRNA microarrays, the nCounter system is inherently multiplexable, with up to $\sim 800$ unique target sequences interrogated on a single chip. Unlike most other multiplexed platforms however, the nCounter human miRNA assay v1 also included 80 viral miRNAs. Subsequent versions have since eliminated the viral miRNAs in favour of additional human miRNAs. 


\subsubsection{4 miRNA-Sequencing}

In line with other miRNA profiling technologies, miRNA-Sequencing (miRNA-Seq) borrows much from its mRNA counterpart, RNA-Seq. RNA- and miRNA-Seq both exploit massively parallel high-throughput (HT) DNA sequencing technologies to gather both sequence and abundance information about the transcriptome of samples analyzed. MiRNA-Seq targets miRNA molecules through specific chemical properties (unique 3' and 5' end modifications), as well as PAGE (polyacrylamide gel-electrophoresis) size fractionation and purification ${ }^{234}$. Following reverse transcription and PCR amplification, size-selected cDNA molecules are run through the HT DNA sequencing platform of choice (e.g. Illumina or ABI SOLiD sequencers). Unless discovery of novel miRNAs is the objective, reads from the sequencing run are mapped to known miRNA sequences, and counting the number of reads which map to a specific miRNA provides the basis for quantification using this method. One major characteristic that sets miRNA-Seq apart from other miRNA measuring techniques is the ability to detect all small RNA molecules in a single reaction, including not only mature miRNAs, but pre-miRNAs, as well as small nucleolar RNAs.

\subsubsection{Comparison of technologies}

While there is no clear-cut "gold-standard" methodology for miRNA profiling, various experimental design characteristics should be considered when selecting the appropriate miRNA expression profiling technology. Factors such as the number and identity of miRNAs interrogated, quantity and quality of RNA required, platform design flexibility, sensitivity, specificity, robustness to contaminants in analyte solution, number of samples to be analyzed, and budget are all variables that must be considered when selecting the appropriate miRNA profiling platform. While several studies have directly compared various miRNA profiling 
platforms, none have included all the available techniques in a single study, and there is no consensus regarding the best method for all scenarios. With these caveats in mind, Table 1-2 summarizes the current knowledge comparing the different platforms as a function of various pertinent factors such as the type and required amount of the starting material, cost, and reported sensitivity and specificity. 
Table 1-2. Comparison of different miRNA profiling platforms and technologies

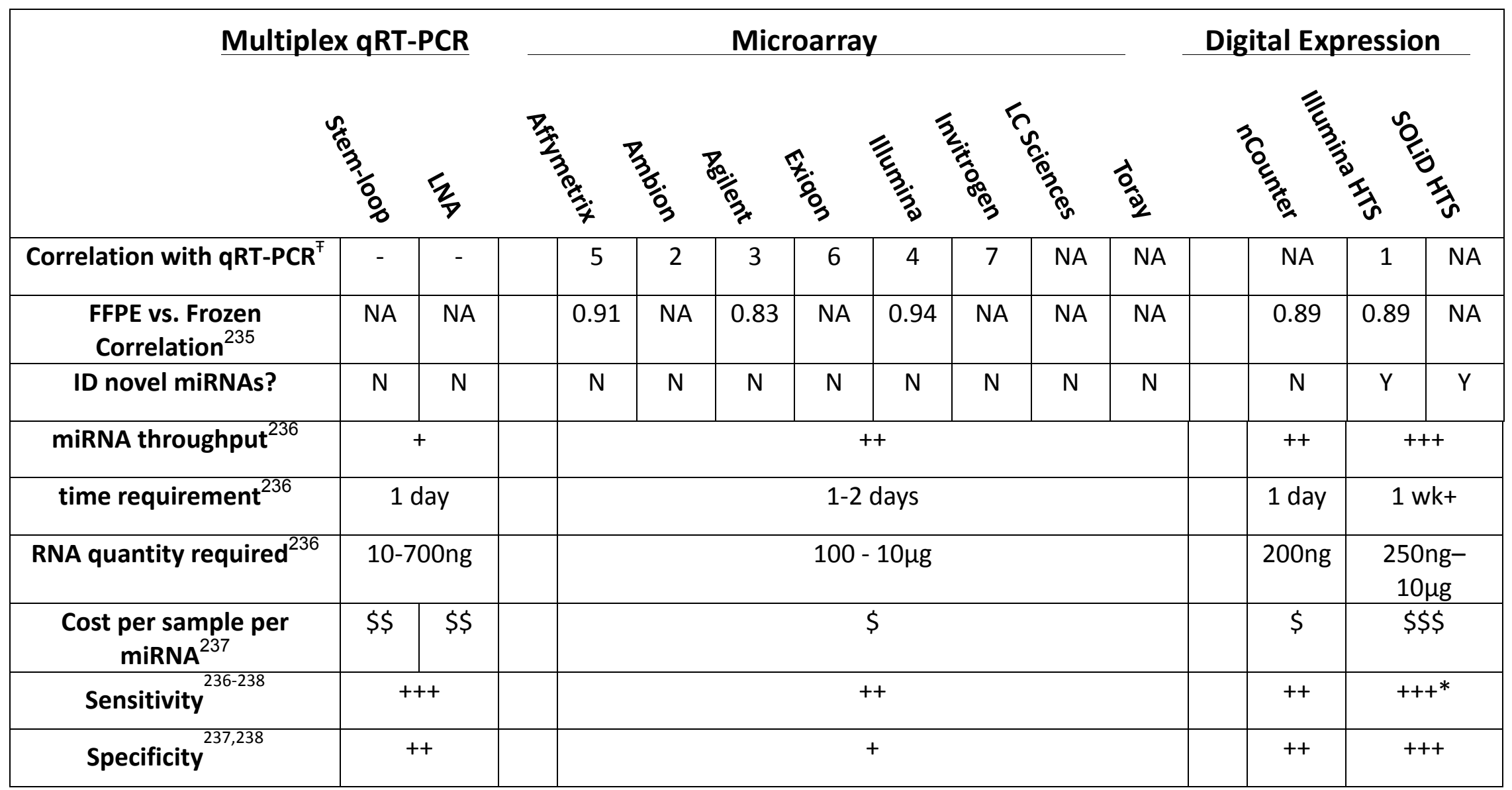

Ғ Six studies ${ }^{235,239-243}$ where direct comparisons were performed between miRNA profiling technologies and single-well qRT-PCR

were used to rank each platform based on its concordance with this widely accepted methodology. Only platforms tested in at least 2 studies were included in the ranking. Platforms were percentile ranked within each study, then their average percentile rank across studies was used to determine their final rank.

* Higher depth of sequencing results in higher sensitivity with the trade off of increased cost and RNA requirements HTS; high-throughput sequencing, NA; not available, Y; yes, N; no.+; low, ++; medium, +++; high 


\subsubsection{6 miRNA expression data analysis}

In order to glean interpretable information from any of the miRNA expression platforms discussed above, the raw data produced by these arrays, cards, and sequencing runs must first be correctly normalized to minimize the effects of systematic experimental variation. Indeed, the normalization methodology used can have a profound impact on the conclusions drawn, reproducibility, and cross-platform agreement ${ }^{244}$. Data processing is an issue that largely needs to be addressed on a case-by-case basis; certainly amongst platforms, and likely amongst various experimental conditions. However, there is no single agreed-upon normalization strategy for any technology; let alone disease or experimental condition.

Similar to the platforms and technologies themselves, miRNA normalization strategies borrow much from their mRNA counterparts. Users of qPCR-based techniques often employ purportedly "invariant" small RNAs, similar to the so-called "house-keeping genes" often used to normalize mRNA qRT-PCR data, as endogenous controls to account for sample-to-sample systematic variation. However, small RNA endogenous controls, most commonly smallnucleolar RNAs (snoRNAs), are subject to biological variation themselves, and as such can distort the data they are meant to be improving ${ }^{245-247}$. Global miRNA expression approaches, such as arrays, chips and sequencing methods allow the user to take advantage of the comprehensive nature of the data obtained, and normalize individual miRNAs against the entire micronome. Numerous mathematical algorithms originally developed for use with mRNA assays such as loess, quantile, variance stabilizing normalization (VSN), global geometric mean, and invariant selection, have all been successfully applied to miRNA platforms ${ }^{244,248-251}$. For miRNA sequencing data, the somewhat simplistic reads-per-million (RPM) technique is still the most widely used normalization method, although, there are multiple others 
available $^{252}$. It is unlikely that there will be one normalization strategy identified that is optimal in all circumstances, even for a given platform. As such, the experimental variables and objectives of the study must always be taken into account when selecting the appropriate data processing methods.

\subsection{Research Objectives}

The majority of NPC patients receive curative RT, with concomitant CRT in cases of locally advanced disease, and these current treatment strategies achieve a modest 5-year survival rate of $\sim 75 \%{ }^{253}$. Moreover, despite recent improvements in local control for NPC patients, distant metastasis remains a major cause of death in this patient population ${ }^{254}$, underscoring a need to better treat and identify those patients at risk of distant metastases. MiRNAs are thought to regulate the majority of protein-coding human genes ${ }^{255,256}$, and it is also apparent that miRNAs play important roles in many, if not all human malignancies ${ }^{257}$. Hence, this thesis proposes to explore NPC biology and clinical behaviour through the lens of miRNAs. The work presented herein can be broken down into three broad Aims:

\section{To identify miRNAs that are dysregulated in NPC.}

2. To characterize the biological cause and impact of dysregulated miRNAs in NPC.

\section{To determine the clinical relevance of dysregulated miRNAs in NPC.}

Chapter 2 presents the identification (Aim 1) and functional characterization (Aim 2) of dysregulated miR-375; whereas, Chapter 3 demonstrates a validated 4-miRNA signature associated with distant metastasis in NPC patients (Aims 1\&3) and preliminary pathway 
enrichment analysis indicating a potential role in cell-cycle control for members of this signature (Aim 2). In addition, Chapter 4 examines copy number alterations in DNA from NPC samples through a comprehensive approach similar to those used to identify aberrantly expressed miRNAs. Notably, several miRNAs were located within frequently altered genomic regions, providing a potential mechanism for miRNA dysregulation in NPC (Aim 2).

In summary, this work provides evidence that miRNAs can provide important insights into both the biology and prognosis of NPC patients. Moreover, the discoveries made herein provide valuable information that could aid in the development of novel targeted therapies and patient stratification methods that will improve outcome for future NPC patients. 


\section{Chapter 2}

\section{Significance of Dysregulated Metadherin and MicroRNA- 375 in Head and Neck Cancer}

A version of this chapter has been published in Clinical Cancer Research 2011 17; 7539

http://clincancerres.aacrjournals.org/cgi/pmidlookup?view=long\&pmid=22031094

Jeff P. Bruce, Angela B.Y. Hui, Nehad M. Alajez, Wei Shi, Shijun Yue, Bayardo PerezOrdonez Wei Xu, Brian O'Sullivan, John Waldron, Bernard Cummings, Patrick Gullane, Lillian Siu, and Fei-Fei Liu

Reprinted with permission from the American Association for Cancer Research. All rights reserved.

Copyright $@ 2011$ Clinical Cancer Research 


\section{Significance of Dysregulated Metadherin and MicroRNA-375 in Head and Neck Cancer}

\subsection{Abstract}

\subsubsection{Purpose}

Despite recent improvements in local control of head and neck cancers (HNCs), distant metastasis remains a major cause of death. Hence, further understanding of HNC biology, and in particular, the genes/pathways driving metastasis is essential in order to improve outcome.

\subsubsection{Experimental Design}

Quantitative RT-PCR was used to measure the expression of miR-375 and metadherin (MTDH) in HNC patient samples. Targets of miR-375 were confirmed using qRT-PCR, western blot analysis and luciferase assays. Phenotypic effects of miR-375 re-expression and MTDH knock-down were assessed using viability (MTS), clonogenic survival, cell migration/invasion, as well as in vivo tumour formation assays. The prognostic significance of miR-375 or MTDH in nasopharyngeal carcinoma (NPC) was determined by comparing low $v s$. high expression groups.

\subsubsection{Results}

MiR-375 expression was significantly reduced $(\mathrm{p}=0.01)$, and conversely, MTDH was significantly increased $(\mathrm{p}=0.0001)$ in NPC samples. Quantitative RT-PCR, western blots and luciferase assays corroborated MTDH as a target of miR-375. Re-expression of miR-375 and siRNA knock-down of MTDH both decreased cell viability and clonogenic survival, cell migration/invasion, as well as in vivo tumour formation. NPC patients whose tumours expressed high levels of MTDH experienced significantly lower survival, and in particular, higher distant relapse rates (5-year distant relapse rates: $26 \% v s .5 \%$; $\mathrm{p}=0.005$ ). 


\subsubsection{Conclusions}

Dysregulation of miR-375 and MTDH may represent an important oncogenic pathway driving human HNC progression, particularly distant metastases, which is now emerging as a major cause of death for HNC patients. Hence, targeting this pathway could potentially be a novel therapeutic strategy by which HNC patient outcome could be improved. 


\subsection{Introduction}

Head and neck squamous cell carcinomas (HNSCC) constitute the $5^{\text {th }}$ most common malignancy worldwide ${ }^{258}$. Patients with locally advanced HNSCC have 5-year overall survival (OS) rates hovering around $30-45 \%{ }^{259}$, underscoring a considerable opportunity for improving outcome. Nasopharyngeal carcinoma (NPC) is another malignancy of the head and neck region; however, NPCs are distinct from other head \& neck cancers (HNCs) due to their unique etiological, clinical/biological and epidemiological characteristics. Locally advanced NPC patients have a 5 -year overall survival rate of $\sim 70 \%^{13}$, also demonstrating a need for improvement. Hence, it is imperative to acquire a deeper understanding of HNC biology to guide the development and evaluation of novel therapies, in order to improve patient outcome.

MicroRNAs (miRNA) are a novel class of gene regulators which are involved in many biological systems, and recognized to play important roles in human cancers ${ }^{260}$. We have recently completed a miRNA profiling study of locally advanced HNSCC, and reported downregulation of miR-375 as one of the most frequently detected aberrations ${ }^{261}$. Preliminary functional analysis demonstrated a potential tumour suppressive role for miR-375 in HNSCC $^{261}$. In the current study, we further investigated the role of miR-375 down-regulation in $\mathrm{HNC}$, and determined that metadherin (MTDH) is a target of miR-375 in HNC. MTDH, also known as astrocyte elevated gene 1 (AEG-1) was only recently cloned ${ }^{262}$; however, it is rapidly emerging as an important oncogene in many human cancers ${ }^{263}$. Furthermore, MTDH overexpression has been observed to significantly correlate with poor prognosis and distant metastases in breast ${ }^{263}$, lung ${ }^{40}$ and gastric ${ }^{264}$ cancers, warranting its further examination in HNC. 


\subsection{Materials \& Methods}

\subsubsection{Patient information \& tissues}

With approval from the Institutional Research Ethics Board (REB), 20 diagnostic formalin-fixed paraffin-embedded (FFPE) blocks were collected from HNSCC patients with locally advanced (Stage III or IV) disease, who were previous participants in a Phase III randomized study of hyperfractionated radiotherapy conducted between $1988-1995^{265}$. Normal epithelial tissues derived from three FFPE blocks of individuals who underwent a tonsillectomy (University Health Network), plus three additional FFPE blocks derived from normal laryngeal squamous epithelial tissues post-laryngectomy (commercially purchased from Asterand, USA) served as controls. In addition, 94 primary FFPE biopsy samples from NPC patients diagnosed from 1993-2000 were evaluated. Eight FFPE blocks of normal nasopharyngeal epithelial tissues derived from patients who underwent a diagnostic quadroscopy served as normal controls. Clinical characteristics of these cancer patients are provided in Table 2-1. 
Table 2-1. Clinical characteristics of HNC patients studied.

\begin{tabular}{|c|c|c|}
\hline \multicolumn{3}{|c|}{ Clinical Descriptors for the 94 NPC Patients } \\
\hline $\begin{array}{l}\frac{\text { Age }}{\text { median }} \\
\text { range } \\
\text { Stage } \\
\text { I } \\
\text { II } \\
\text { III } \\
\text { IV } \\
\text { Relapse } \\
\text { No relapse } \\
\text { Local relapse only } \\
\text { Local + Distant relapse } \\
\text { Distant relapse only } \\
\text { Treatment } \\
\text { Radiation only } \\
\text { Radiation + chemo }\end{array}$ & $\begin{array}{l}46 \\
16-79 \\
\text { Frequency } \\
8 \\
32 \\
28 \\
26 \\
\text { Frequency } \\
64 \\
7 \\
13 \\
10 \\
\text { Frequency } \\
69 \\
25\end{array}$ & $\begin{array}{l}\text { Percent } \\
8 \% \\
34 \% \\
30 \% \\
28 \% \\
\text { Percent } \\
68 \% \\
7 \% \\
14 \% \\
11 \% \\
\text { Percent } \\
73 \% \\
27 \%\end{array}$ \\
\hline \multicolumn{3}{|c|}{ Clinical Descriptors for the 20 HNSCC Patients } \\
\hline $\begin{array}{l}\frac{\text { Age }}{\text { median }} \\
\text { range } \\
\text { Stage } \\
\text { III } \\
\text { IV } \\
\text { Relapse } \\
\text { No relapse } \\
\text { Local relapse } \\
\text { Regional relapse } \\
\text { Local + regional } \\
\text { relapse } \\
\text { Treatment } \\
\text { Radiation \#1 (D) } \\
\text { Radiation \#2 (T) } \\
\text { (T = twice daily) and (D }\end{array}$ & $\begin{array}{l}59 \\
35-75 \\
\text { Frequency } \\
8 \\
12 \\
\text { Frequency } \\
4 \\
6 \\
4 \\
6 \\
\text { Frequency } \\
9 \\
11 \\
\text { (dard) }\end{array}$ & $\begin{array}{l}\text { Percent } \\
40 \% \\
60 \% \\
\text { Percent } \\
20 \% \\
30 \% \\
20 \% \\
30 \% \\
\\
\text { Percent } \\
45 \% \\
55 \%\end{array}$ \\
\hline
\end{tabular}




\subsubsection{RNA purification from FFPE samples}

To ensure that all tissues analyzed contained $>70 \%$ tumour cells, a representative section from each patient was stained with $\mathrm{H} \& \mathrm{E}$, then reviewed by a $\mathrm{HNC}$ pathologist (B P-O) to ascertain regions with malignant epithelial cells for macro-dissection. All blocks were processed randomly, with clinical outcome unknown, to avoid experimental bias. Total RNA enriched for small RNA species was isolated using the RecoverAll ${ }^{\mathrm{TM}}$ Total Nucleic Acid Isolation Kit for FFPE (Ambion, Inc.), according to the manufacturer's instructions.

\subsubsection{Cell lines and reagents}

The human hypopharyngeal HNSCC cell line, FaDu, was obtained from American Type Culture Collection (Manassas, VA), and cultured according to specifications. The Epstein-Barr virus (EBV) positive NPC cell line C666- ${ }^{31}$ was maintained in RPMI 1640 supplemented with $10 \%$ fetal bovine serum (Wisent, Inc.), and $100 \mathrm{mg} / \mathrm{L}$ penicillin/streptomycin. The normal oral epithelial (NOE) cells (Celprogen, Inc.) served as normal controls. All cells were authenticated at the Centre for Applied Genomics (Hospital for Sick Children, Toronto, Canada) using the AmpF/STR Identifiler PCR Amplification Kit (Applied Biosystems), and maintained at $37^{\circ} \mathrm{C}$ with $5 \% \mathrm{CO}_{2}$.

\subsubsection{5-aza-2'-deoxycytidine treatment and quantitative CpG methylation analysis}

C666-1 and FaDu cells were seeded overnight in 12-well plates and media containing $3 \mu \mathrm{M}$ 5-aza-2'-deoxycytidine (Sigma Aldrich) was added to cells 2 and 4 days after seeding, respectively. On day 5, cells were harvested and RNA was extracted for qRT-PCR analysis of 
miR-375 expression levels. The CpG islands in the genomic region of miR-375 were identified using the EMBL-EBI CpG Island Finder and Plotting Tool (Fig 2-1A) and 3 amplicons spanning these regions (Fig 2-1B) were analyzed for CpG methylation using the EpiTYPER (Sequenom, Inc.) at the Analytical Genetics Technology Centre (AGTC), University Health Network, Toronto. 
A

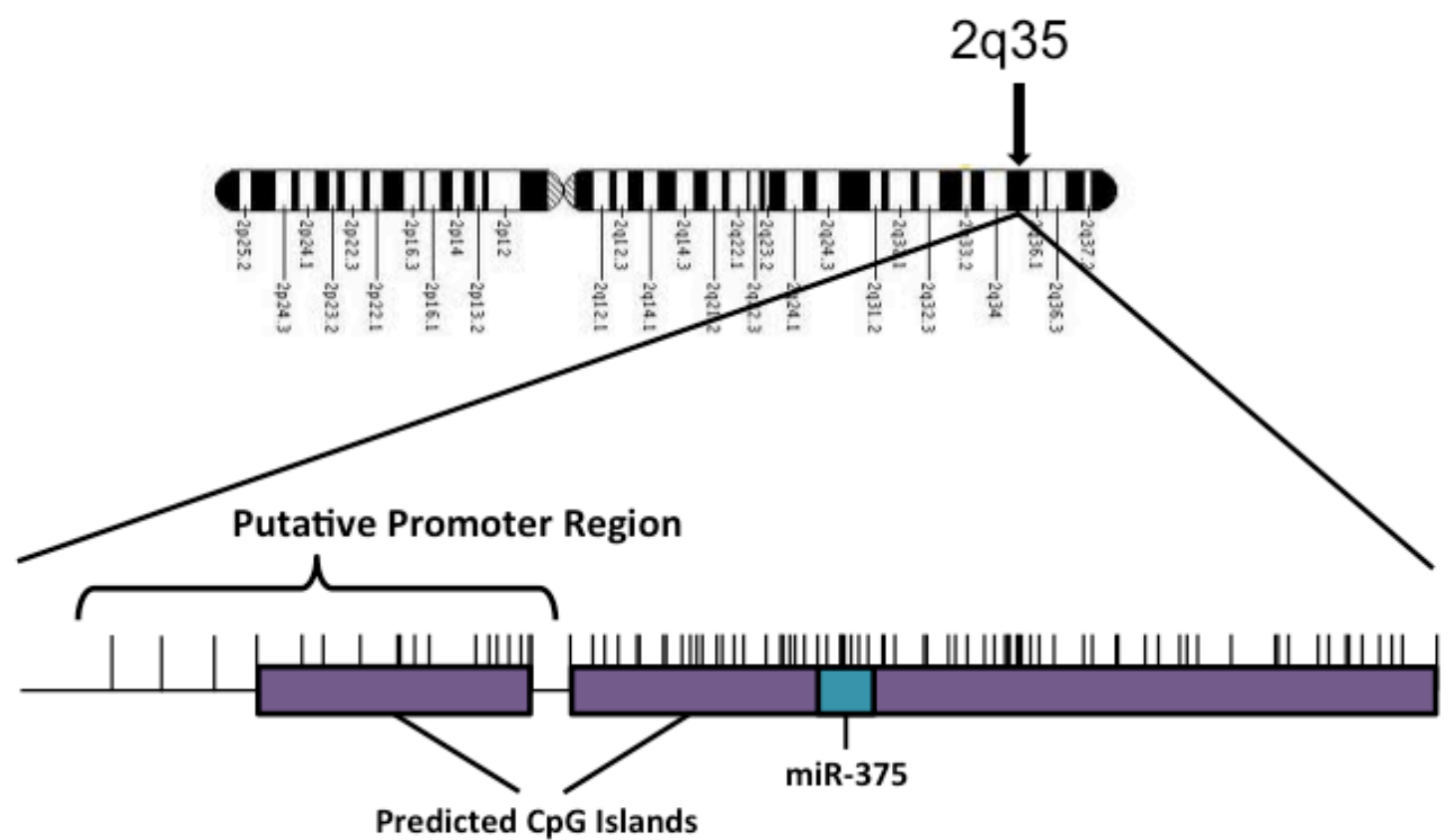

B

Amplicon Sequences:

GACCAAGGGAGTGTCAGGGAGTGACATCTCAGGATGTCAGCAGCACCACAGAGATCCGAGGAGTGGATT TGAGGCTCCATGGTGGCCCTCAGCTTCCCCCGCCCAGATTTTTCCACACCTCAGCCTTGGCTCCAACTCCG TGAGGGCCTGCATCTTCCCCAGCATGACCAGGAGCCCAGGAACCGGGACAGGAGGCTGGGGAGGGCCT AGCCCCTGCGGGAGGCCAGACCAACCGTTCACTGAGCAAATGGGAAAACCAAGAGGCCGGGAAATGACT TGCCCAAGGCCATAGAAGTCGCGGGGACAGAGGCGGACTAGAATCCGGGTTTCCACCTCCAGAAGGGTT TCTGTTCTGGGCCACGGCCCCCTTCGCCACCCGCTGGAGCCGGCTCAGCCCGTATTACGACGCAGAATG GAGCCTGGCAGCCCAGAAGCTGCGGGCAGGGCAAGGCAGGGCGGGTGGGGCCGGATGGGAGCCGGAT CTCCCCTGGAGCGGCGCACAGCCTCTCCCACCCGTACGGTTGAGATGGCGGTGGCGGTGGCGGCGGCA GGTGCCTGCGTGGCGATCAGGCCGGCCCCCGGGCTGGTGCTGAGAGGCCGCCCCTGCCTCACGCGAG CCGAACGAACAAAACGCTCAGGTCCGGTTTGTGCGAGGGGCTCGTCGCGGGGCGGGAGCGGAGGGCC CGGGGCCTGGCGGTCGCCCTCGGTGATCTCCTGGTCCTGGTCTTCCGCGAGCTCAGTGCTGCACAGCG CCCCGCTAGCCTCCGCCCTCACCTGAACGCATCTGCGGCTGACACGTCGGCGGCCGCGGCGGCCACCG CAGGTGCGCACCTGGCCCGCCCCTCCTTTCCCAGCCACCCTCCGCCCGGCTTTCCTGGCCCCTCCGCC CGGCCCAGCCTAGCTTGGCTTCGTCCCCAGCCCACGCCTTGGAGCTTGTCCCGGAGCCCGGGCTCCGT GTGCTCTTATACTGCCTGTTCAGTCCCCGCCCCCAGACACACACACTCACACCTGTCCAGGCGCCCGTTT GCTCGGGGCAAATATTGACTCATGGAGGCGGAGCGCTCCCCCCTCCCCCGCCCGGGCTCTGAGACCGG AGCTTCTCCGAGGCCTTGGTGGCGGGGGCGGAGGGGAGGGTGCTGGGGACCCTG

Amplicon \#1 (blue): $\quad$ Length: 355 bp

Amplicon \#2 (pink): $\quad$ Length: 489 bp

Amplicon \#3 (green): $\quad$ Length: 442 bp

Note: Sequence marked in yellow represent regions of overlap between amplicons.

Figure 2-1. Genomic location of miR-375 at chromosome $2 q 35$.

The $\mathrm{CpG}$ islands in the genomic region of miR-375 were identified using the EMBL-EBI CpG Island Finder and Plotting tool. 


\subsubsection{Quantification of miRNA and mRNA}

MicroRNA expression was assessed by qRT-PCR analysis using TaqMan ${ }^{\circledR}$ microRNA Assays (Applied Biosystems) as previously described ${ }^{266}$. Quantitative RT-PCR was also utilized to analyze expression changes of four previously published miR-375 targets: MTPN, USP1, PDK1, ADIPOR2, plus five newly identified miR-375 targets: MTDH, GMFB, RANBP3, SPAG9 and ZNF462. Total RNA was isolated from cells using the Total RNA purification Kit (Norgen, Inc.). One $\mu$ g of total RNA was reverse-transcribed using SuperScript II Reverse Transcriptase (Invitrogen Canada) as specified by the manufacturer. Quantitative RT-PCR was performed using SYBR Green PCR Master Mix (Applied Biosystems) and an ABI PRISM 7900 Sequence Detection System (PerkinElmer Biosystems). Primers for PCR amplifications (Table 2-2) were designed using Primer 3 Input (version 0.4.0). Relative mRNA levels were calculated using the $2^{-\Delta \Delta \mathrm{Ct}}$ method $^{267}$. 
Table 2-2. Primer sequences for qRT-PCR of potential miR-375 targets.

\begin{tabular}{|c|c|c|}
\hline Gene Symbol & Forward Primer & Reverse Primer \\
\hline SPAG9 & 5'-GCTGACCAGATTAGCAGACTTGA -3' & 5'-GGATTCTAGTTGATCACTCCCTGA -3' \\
\hline MTDH & 5'-GATGATGAATGGTCTGGGTTAAA -3' & 5'- GACCTTTTGATCATCAGGAATTG-3' \\
\hline USP1 & 5'-GACAAGGGAAATTGCAAAGA-3' & 5'-TGTGTTAAGCAGTCGCCTTG-3' \\
\hline ZNF462 & 5'- CTCAAAGCTCATGCTGGTGA-3' & 5'- GTGGGAGTGTGAGGAGAGGA-3' \\
\hline MTPN & 5'-GCCAAGGGAGAAGATGTCAA-3' & 5'-AGCACCCTTTGACAGAAGCA-3' \\
\hline GMFB & 5'- TAAACGCCTGGTGGTACTGG-3' & 5'-TCAGGCTTACATCCAACAGGA -3' \\
\hline RANBP3 & 5'-GAGAACTGGCAGGGAGGTC -3' & 5'-СCACTGCTCCTTTCCTCTGA-3' \\
\hline YAP1 & 5'- CGTTTTGCCATGAACCAGAG-3' & 5'-GCAGCCTCTCCTTCTCCATC -3' \\
\hline AEBP2 & 5'- GTCAGCGAGGAGGGGTATTT-3' & 5'-CATTGCAGCCACCAACAA -3' \\
\hline PDK1 & 5'-ACATCTGTAGGTTTTGGTTTCCA-3' & 5'-AAAGACACGTGCAATGATGG-3' \\
\hline ADIPOR2 & 5'-TTTGTTTGTAAGGTATGGGAAGG-3' & 5'-TGTGTCCAAATGTTGCCTGT-3' \\
\hline
\end{tabular}




\subsubsection{Construction of plasmids and luciferase assays}

Wild-type and/or mutant fragments (150-200 bp) from the 3'UTR of MTDH, MTPN and USP1 containing predicted binding sites for miR-375 were individually amplified by AmpliTaq gold DNA polymerase (Applied Biosystems) using the primers listed in Table 2-3. The PCR products were subsequently purified, digested with SPEI and HINDIII, and then cloned downstream of the firefly luciferase gene in the pMIR-REPORT vector (Ambion, Inc.) to produce the following plasmids: pMIR-MTDH, pMIR-MTDH-MUT, pMIR-MTPN, and pMIR-USP1. Subsequently, FaDu and C666-1 cells were seeded onto 24-well plates one day and three days before transfection, respectively. Cells were then transfected with $100 \mathrm{nM}$ of pre-miR-NEG or pre-miR-375 in the morning; 6 hours later, cells were co-transfected with 100 ng of the reporter plasmid of interest, plus $50 \mathrm{ng}$ of pRL-SV40 (Promega BioSciences) containing the renilla luciferase gene. Luciferase activity was measured 48 hours posttransfection using the Dual-Glo luciferase assay system according to manufacturer's instructions (Promega BioSciences). Firefly luciferase activity was normalized to that of the renilla luciferase. 
Table 2-3. Primer sequences used for cloning and screening of pmiR-REPORT luciferase encoding vectors.

\begin{tabular}{|c|c|c|}
\hline Gene & Forward Primer & Reverse Primer \\
\hline \multirow[t]{2}{*}{ MTDH } & $\begin{array}{l}\text { GTTGTTACTAGTATGTTGTCATTATATT } \\
\text { TGACTGTGGTT }\end{array}$ & $\begin{array}{l}\text { GTTGTTAAGCTT AAGGCCAGTCTTC } \\
\text { AGCAAAA }\end{array}$ \\
\hline & $\begin{array}{l}\text { Reverse screen: } \\
\text { AACCATTTTGTTCGTTTAGCTTTC }\end{array}$ & \\
\hline \multirow[t]{2}{*}{$\begin{array}{l}\text { MTDH- } \\
\text { mutant }\end{array}$} & $\begin{array}{l}\text { GTTGTTACTAGTATGTTGTCATTATATT } \\
\text { TGACTGTGGTT }\end{array}$ & $\begin{array}{l}\text { AAGGCCAGTCTTCAGCAAAACTAAA } \\
\text { ACCATTCGTCGATTTTAGCTTTCCTA } \\
\text { GTCTGAC }\end{array}$ \\
\hline & $\begin{array}{l}\text { Reverse screen: } \\
\text { GTTGTTAAGCTTAAGGCCAGTCTTCA } \\
\text { GCAAAACTAAAACCATTCGTCGATTTT } \\
\text { AGCTTTCCTAGTCTGAC }\end{array}$ & \\
\hline \multirow[t]{2}{*}{ USP1 } & AGTGAAATTTGAATTACTGAAAACCA & AAGCAAACACAAAAGACTGGTG \\
\hline & $\begin{array}{l}\text { Reverse screen: } \\
\text { TGGTGTAATCCAAGTGTTTAAATG }\end{array}$ & \\
\hline \multirow[t]{2}{*}{ MTPN } & GACTAGTGCTTTGTGCATTTTGA & TGTAGCACAATTCAAGTTTATTCCA \\
\hline & $\begin{array}{l}\text { Reverse screen: } \\
\text { CAAGTTTATTCCATTTGTTCTTGC }\end{array}$ & \\
\hline
\end{tabular}




\subsubsection{Viability and clonogenic assays}

Viability of pre-miR-375, or siMTDH-transfected FaDu or C666-1 cells was examined using the CellTiter 96® Non-Radioactive Cell Proliferation Assay (MTS), according to the manufacturer's protocol (Promega BioSciences). The cellular effects of these manipulations were further investigated in $\mathrm{FaDu}$ cells using clonogenic assays as previously described ${ }^{266}$. Briefly, cells were re-seeded at low density in 6-well plates in triplicate, and incubated at $37^{\circ} \mathrm{C}$ under 5\% $\mathrm{CO}_{2}$ at 3 days post-transfection. After 10-12 days, plates were washed, fixed in 50\% methanol and stained with $0.1 \%$ crystal violet, and then the number of colonies was counted. The fraction of clonogenically viable cells was calculated by comparison of pre-miR-375 or siMTDH transfected cells with pre-miR-NEG or scrambled siRNA (siNEG) transfected cells, respectively.

\subsubsection{Cell cycle analysis}

Cell cycle analysis of pre-miR-375 transfected C666-1 cells was performed as previously described ${ }^{266}$. Briefly, cells were harvested and washed with fluorescence-activated cell sorting (FACS) buffer (PBS/0.5\% BSA), then re-suspended and fixed with ice-cold 70\% ethanol. After washing, cells were re-suspended in FACS buffer and incubated with propidium iodide in the dark before analysis in the BD FACScalibur using the FL-2 channel. The flow cytometry data were analyzed using FlowJo software (Tree Star, Inc.).

\subsubsection{Western blot analysis}

Total protein extracts were harvested from cell lines and prepared for immunoblotting as previously described ${ }^{268}$. Membranes were probed with anti-Phospho-Akt (Ser473) (D9E) XP ${ }^{\circledR}$ 
rabbit mAB (Cell Signaling Technology), anti-Akt (pan) (C67E7) rabbit mAB (Cell Signaling Technology), anti-MTDH polyclonal (clone L-19; 1:1000 dilution; Invitrogen, Inc.), anti- $\beta$ actin (8H10D10) mouse mAB (1:20,000 dilution; Cell Signaling Technology), or anti-GAPDH monoclonal antibodies (1:15,000 dilution; Abcam, Inc.), followed by secondary antibodies conjugated to horseradish peroxidase (1:2000 dilution; Abcam, Inc.) or IRDye ${ }^{\circledR}$ fluorescent Secondary Antibodies (1:20,000 dilution; LI-COR Biosciences). GAPDH or $\beta$-actin protein levels were used as loading controls. Western blots were quantified with the Adobe Photoshop Pixel Quantification Plug-In (Richard Rosenman Advertising \& Design, Toronto, Canada), or the Odyssey Application Software 2.1 (Li-cor Biosciences).

\subsubsection{In vitro migration and invasion assays}

Invasion and migration of $\mathrm{HNC}$ cells were assayed using the BD BioCoat ${ }^{\mathrm{TM}}$ Matrigel $^{\mathrm{TM}}$ Invasion Chambers and Control Inserts (BD Bioscience), respectively. Each well of a 24-well plate contained an insert with an $8 \mu \mathrm{m}$ pore size PET (polyethylene terephthalate) membrane. Inserts coated with a thin layer of matrigel basement membrane matrix were used to measure the ability of the cells to invade through the reconstituted basement membrane. For both migration and invasion assays $1 \times 10^{5}$ cells were seeded inside the insert with medium containing $0.5 \%$ serum. High serum $(20 \%)$ medium was then added to the bottom chamber of 24 -well plates to serve as a chemo-attractant. After $48 \mathrm{hrs}$, the membranes were washed, stained, then separated with a sterile scalpel and mounted on a glass slide. The number of migrating or invading cells was then counted under a light microscope. 


\subsubsection{Tumour formation assay}

All animal experiments utilized 6-8 week-old severe combined immunodeficient (SCID) $\mathrm{BALB} / \mathrm{c}$ female mice in accordance with the guidelines of the Animal Care Committee, Ontario Cancer Institute, University Health Network (Toronto, Canada). Cells were first transfected with siNEG, siMTDH, pre-miR-NEG, or pre-miR-375 $72 \mathrm{hrs}$ before they were harvested and their viability was assessed by the trypan blue exclusion method. Subsequently, $2.5 \times 10^{5}$ viable cells were suspended in $100 \mu \mathrm{L}$ of growth medium, and injected intramuscularly into the left gastrocnemius muscle of female SCID mice. Tumour growth was monitored by measuring tumour plus leg diameter (TLD) three times a week. Mice were euthanized by $\mathrm{CO}_{2}$ once TLDs reached $14 \mathrm{~mm}$.

\subsubsection{Immunohistochemical (IHC) detection of MTDH expression}

Protein expression of MTDH was evaluated in 94 NPC patient samples using immunohistochemistry, which was performed on $5 \mu \mathrm{m}$ FFPE sections of each patient's tumour using microwave antigen retrieval, in combination with the Level-2 Ultra Streptavidin system (Signet Laboratories). MTDH expression was detected with the rabbit polyclonal anti-MTDH antibody (1:50 dilution; Invitrogen, Inc.). Expression levels of MTDH were graded according to staining intensity.

\subsubsection{Statistical analyses}

All experiments were performed at least three independent times, with the data presented as the mean \pm SEM. The statistical differences between treatment groups were determined using a Student's $t$ test when comparing two treatment groups, or a one-way 
ANOVA followed by Tukey's method when comparing more than two treatment groups. Statistical analyses and graphing were performed using Microsoft excel and Graphpad Prism software (Graphpad Software, Inc.).

To determine the potential prognostic significance of miR-375 or MTDH expression, NPC patients were dichotomized into low $(<$ median) or high $(\geq$ median $)$ expression groups which were compared based on several clinical parameters: overall survival, disease-free survival, local relapse-free survival, nodal relapse-free survival, or distant relapse-free survival. Median follow-up time for this cohort was 3 years. Using the log-rank test, a p-value $<0.05$ was considered significant.

\subsection{Results}

\subsubsection{Down-regulation of miR-375 in HNC cell lines and primary NPC patient} samples

We have previously reported a high frequency of miR-375 down-regulation in HNSCC samples $^{261}$. To further validate the importance of this miRNA in HNC, expression of miR-375 was measured in an additional seven HNC cell lines, and compared to that of the normal oral epithelial (NOE) cell line (Fig 2-2). Down regulation of miR-375 was noted in five of the seven HNC cell lines analyzed (UTSCC-42a, VU147T, HONE-1, HK-1 and C666-1); the C666-1 cells were observed to have the lowest level of miR-375 expression ( $\sim 16$-fold lower than NOEs). We previously reported that pre-miR-375 transfection of FaDu cells inhibited cell growth, along with a modest increase in the sub- $\mathrm{G}_{0} / \mathrm{G}_{1}$ population ${ }^{261}$. These same analyses demonstrated that miR-375 transfection in C666-1 cells caused a similarly significant reduction in viability to $\sim 60 \%$ by day 3 , maintained for at least 6 days (Fig 2-3A). Cell cycle analysis 
corroborated that transfection of C666-1 cells with pre-miR-375 resulted in a significant increase in the sub- $\mathrm{G}_{0} / \mathrm{G}_{1}$ fraction compared to cells transfected with pre-miR-NEG (11.6\% vs. $1.5 \% ; \mathrm{p}=0.002)$; no other phases of the cell cycle appeared to be affected (Fig 2-3B). The expression level of miR-375 was then examined in 92 FFPE NPC compared to 8 normal nasopharyngeal epithelial samples. This analysis demonstrated significantly lower miR-375 expression in NPC vs. normal samples (Fig 2-3C; $\mathrm{p}=0.01$ ). Using a cut-off of 2-fold below the mean expression of normal nasopharyngeal epithelial tissues, miR-375 was under-expressed in 69 out of $92(75 \%)$ NPCs; its down-regulation however, was not significantly associated with clinical outcome (Fig 2-4). 


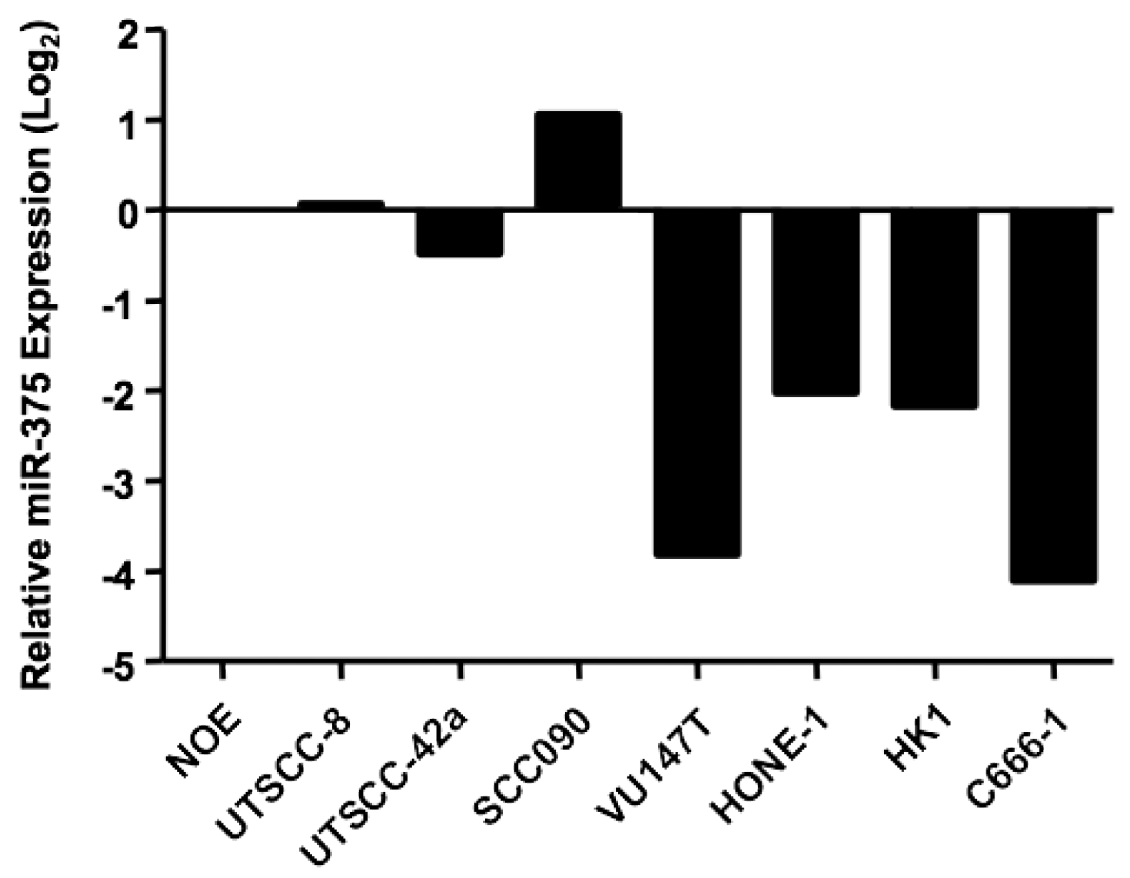

Figure 2-2. miR-375 expression in HNC cell lines.

Quantitative RT-PCR analysis of miR-375 expression in $7 \mathrm{HNC}$ cell lines, compared to that of the normal oral epithelial (NOE) cell line. Relative expression was calculated by normalizing to RNU44 and RNU48 expression, in comparison to that of NOEs. 


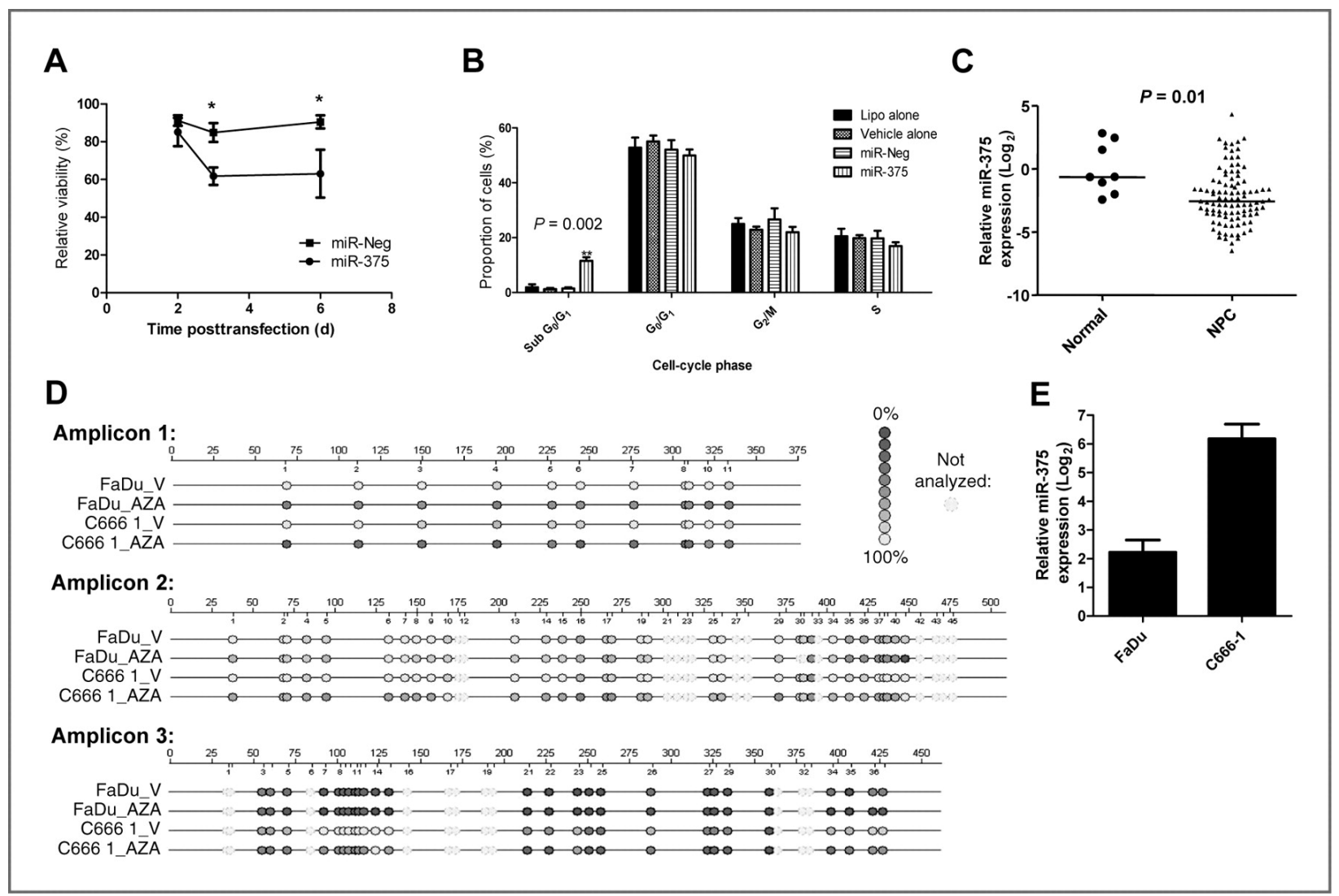

Figure 2-3. Down-regulation of miR-375 in HNC cells

(A) Viability of C666-1 cells as assessed by the MTS assay 3 and 6 days post-pre-miR-375 or pre-miR-NEG transfection. (B) Cell cycle analysis as performed by flow cytometry on C666-1 72 hours post-pre-miR-375 or pre-miR-NEG transfection. (C) MiR-375 expression in 92 primary NPC biopsy samples, compared to 8 normal nasopharyngeal epithelial control tissues as measured by qRT-PCR. (D) Bisulfite sequencing analysis of the CpG sites within a $\sim 1200 \mathrm{bp}$ region around the genomic locus encoding miR-375. Methylation status of three amplicons was analyzed on C666-1 and $\mathrm{FaDu}$ cells, treated with either 5-aza-2'-deoxycytidine (FaDuAZA/C666-1-AZA), or DMSO (FaDu-V/C666-1-V). (E) qRT-PCR analysis of miR-375 expression in $\mathrm{FaDu}$ and $\mathrm{C} 666-1$ cells after treatment with 5-aza-2'-deoxycytidine relative to vehicle-control (DMSO) treated cells. Each experiment was performed at least three independent times, with the data presented as the mean \pm SEM. *indicates $p<0.05$ 
A

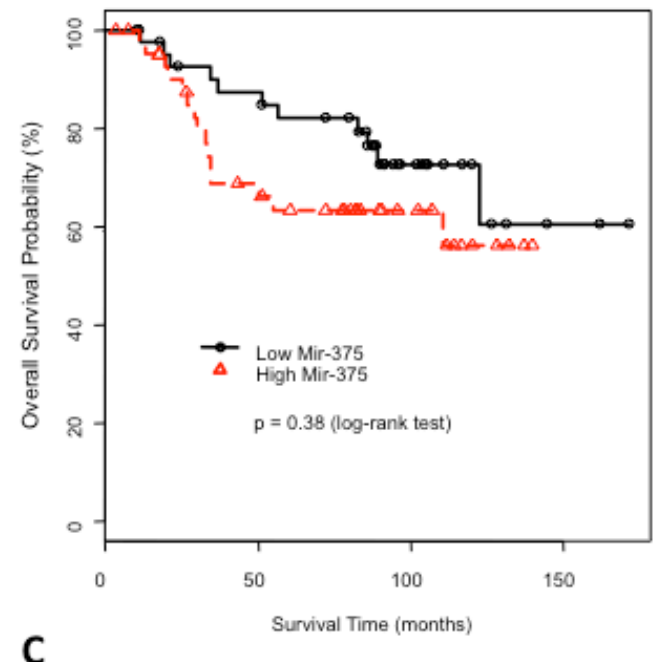

C
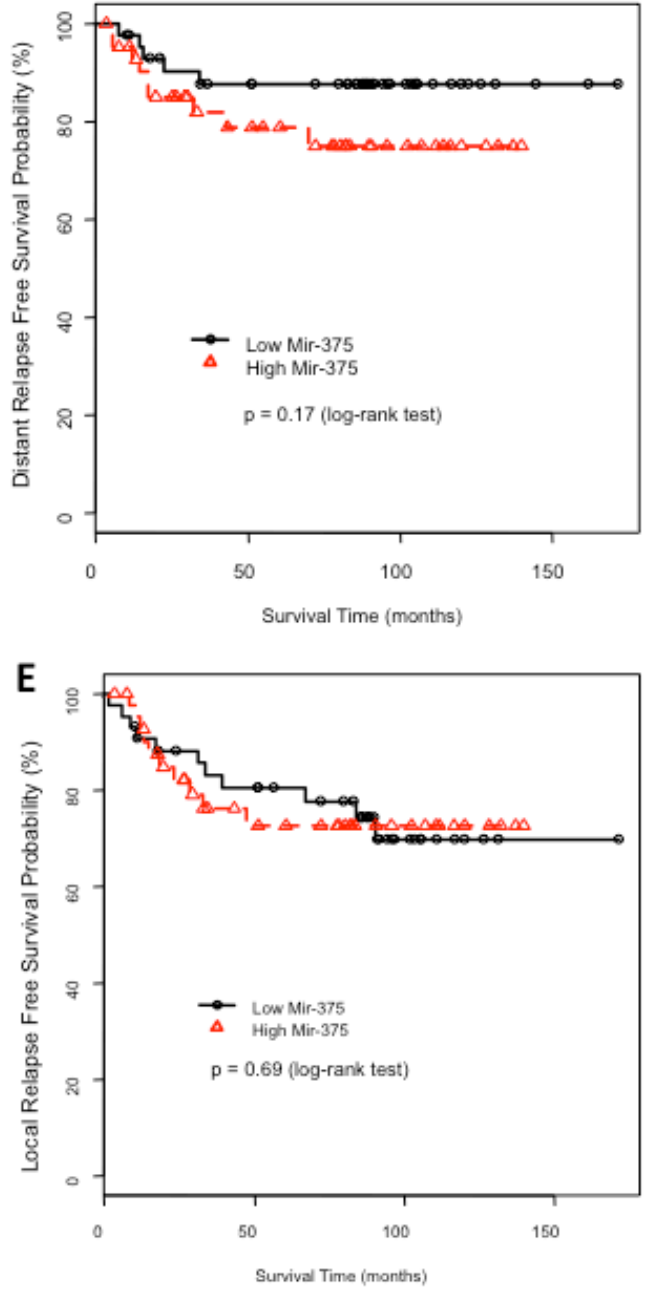

B

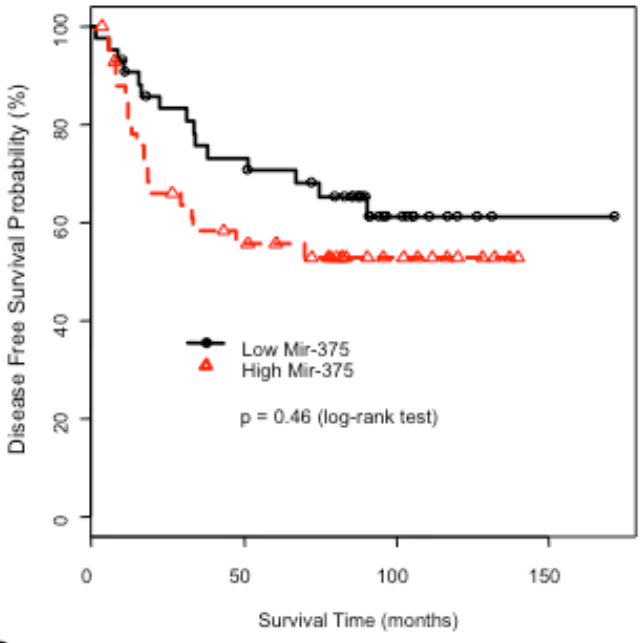

D

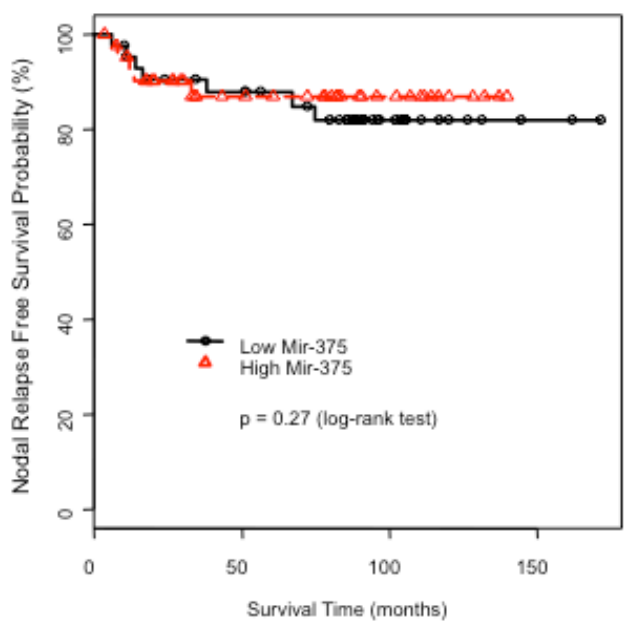

Figure 2-4. Prognostic significance of miR-375 expression in NPC patient samples. Kaplan-Meier plots dichotomized based on miR-375 transcript expression level for NPC patients. Plots show (A) overall; (B) disease-free; (C) distant relapse-free; (D) nodal relapsefree; and (E) local relapse-free survival. 


\subsubsection{Methylation status of the genomic region containing miR-375}

Methylation has been reported as a potential mechanism of miR-375 silencing in hepatocellular carcinoma $(\mathrm{HCC})^{269}$; hence we examined this possible mechanism in both $\mathrm{FaDu}$ and C666-1 cells using both bisulfite sequencing and methylation-status related expression analysis. Bisulfite sequencing conducted on three amplicons spanning the genomic region previously reported to be methylated in $\mathrm{HCC}^{269}$ demonstrated that $\sim 73 \%$ and $65 \%$ of the $\mathrm{CpG}$ sites in this area were indeed methylated in C666-1 and FaDu cells, respectively (Fig 2-3D). Furthermore, treatment of both cell lines with the global de-methylating agent 5-aza-2'deoxycytidine (5-aza) significantly reduced methylation at all $\mathrm{CpG}$ sites (Fig 2-3D), which was associated with the subsequent re-expression of miR-375 (Fig 2-3E), clearly indicating that one mechanism for miR-375 suppression in HNC cells is hyper-methylation of the promoter or coding regions of miR-375.

\subsubsection{Identification of mRNA targets of miR-375}

To identify the mRNA targets of miR-375, genome wide mRNA expression analysis was performed comparing pre-miR-375 transfected HNC cells with that of pre-miR-NEG transfected cells. Transcripts with $>2$-fold down-regulation in pre-miR-375 transfected cells were compared to in silico predicted targets from 7 publicly available databases (miRanda, miRDB, RNA22, PITA, miR-Walk, RNAHybrid, TargetScan) generating a list of nine candidate target mRNAs (Table 2-4). As confirmation of the validity of our methodology, two of these genes (MTPN, USP1) had been previously reported as targets of miR-375 $5^{171,270}$. The effect of miR-375 transfection on gene expression was analyzed for 6 of the 7 identified genes (optimal primers for AEBP2 could not be generated), as well as two previously reported miR- 
375 targets (PDK1, ADIPOR2; ${ }^{171,271}$ ). Transfection of FaDu and C666-1 cells with pre-miR375 led to down-regulation of all potential mRNA targets with the exception of ADIPOR2 in FaDu cells (Fig 2-5). Of note, MTDH was consistently down-regulated to the lowest level in both HNC cell lines; moreover, MTDH was the only target predicted by all seven in silico algorithms (Table 2-4).

Table 2-4. Potential miR-375 targets identified using both DNA micro-arrays and in silico prediction algorithms.

\begin{tabular}{|llll|}
\hline Gene Symbol & Fold Change & Location & $\begin{array}{l}\text { Number of } \text { In } \\
\text { silico predictions }\end{array}$ \\
\hline SPAG9 & 0.349 & $17 q 21.31$ & 1 \\
\hline MTDH & 0.373 & $8 q 22.1$ & 7 \\
\hline USP1 & 0.388 & $1 p 31.3$ & 2 \\
\hline ZNF462 & 0.418 & $9 q 31.2$ & 4 \\
\hline MTPN & 0.421 & $7 q 33$ & 3 \\
\hline GMFB & 0.444 & $14 q 22.2$ & 2 \\
\hline RANBP3 & 0.46 & $19 p 13.3$ & 3 \\
\hline YAP1 & 0.461 & $11 q 13$ & 1 \\
\hline AEBP2 & 0.465 & $12 p 12.3$ & 4 \\
\hline
\end{tabular}




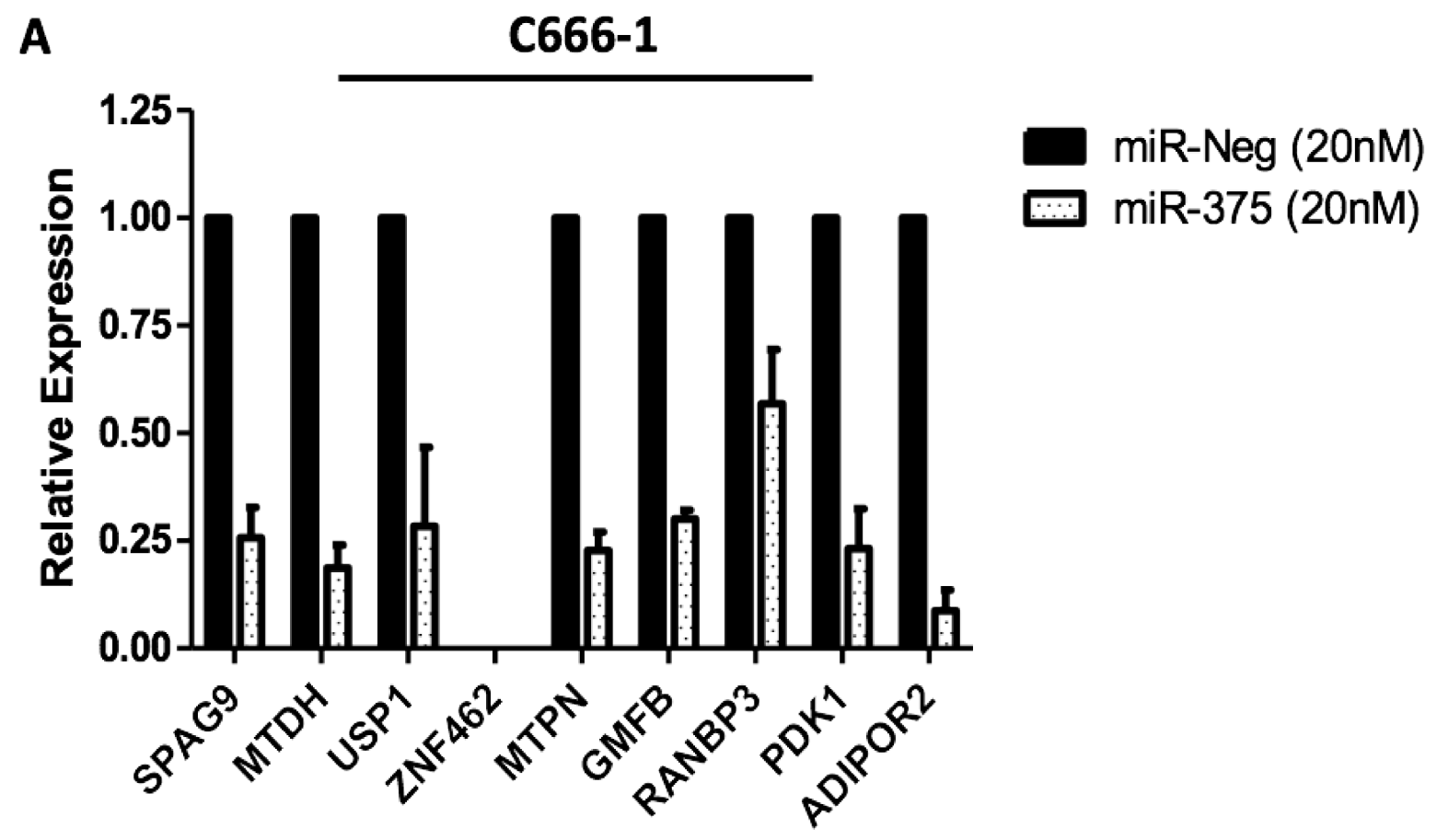

B
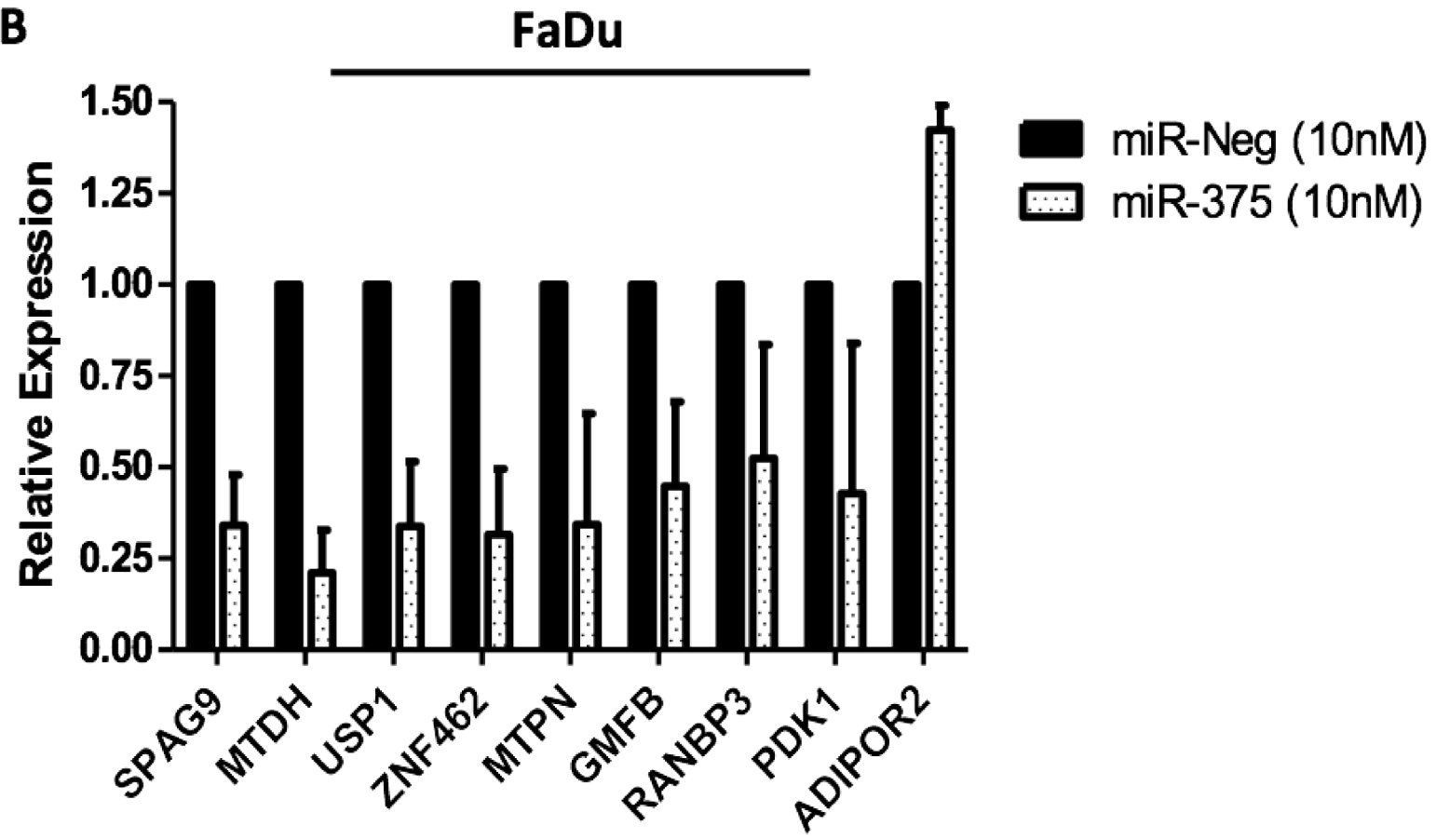

Figure 2-5. Expression of putative targets in HNC cells after miR-375 transfection.

Quantitative RT-PCR analysis of candidate miR-375 mRNA targets for (A) C666-1 cells; and (B) FaDu cells transfected with miR-Neg or miR-375 (20 nM each), assayed 48 hrs or 24 hours post-transfection for C666-1 and FaDu cells, respectively. Relative expression was calculated by normalizing to GAPDH expression, in comparison to cells transfected with pre-miR-NEG. 
Down-regulation of MTDH by miR-375 was confirmed at both the transcript and protein level by qRT-PCR and Western blot analysis, respectively (Fig 2-7A). The direct interaction between miR-375 and MTDH was then verified using luciferase assays. Figure 2-6 depicts the in silico predicted binding sites between the miR-375 seed region with the MTDH transcript. The predicted binding site located at nucleotides 3518-3539 in the 3'UTR of the MTDH transcript was supported by all seven in silico databases and was therefore selected for plasmid construction. In comparison to control cells transfected with pMIR-REPORT, FaDu and C666-1 cells transfected with pMIR-REPORT-MTDH demonstrated a significant reduction in luciferase activity of $18 \%$ and $20 \%$ respectively, when co-transfected with pre-miR-375 (Fig 2-7B). Upon mutating the miR-375 binding site, this inhibitory effect was completely abrogated, corroborating that the observed decrease in luciferase activity was indeed dependent upon an intact miR-375 binding site. Two previously reported mRNA targets, MTPN and USP1, were also demonstrated to be potential targets of miR-375 in both HNC cells (Fig 2-8). 


\section{MTDH mRNA:}

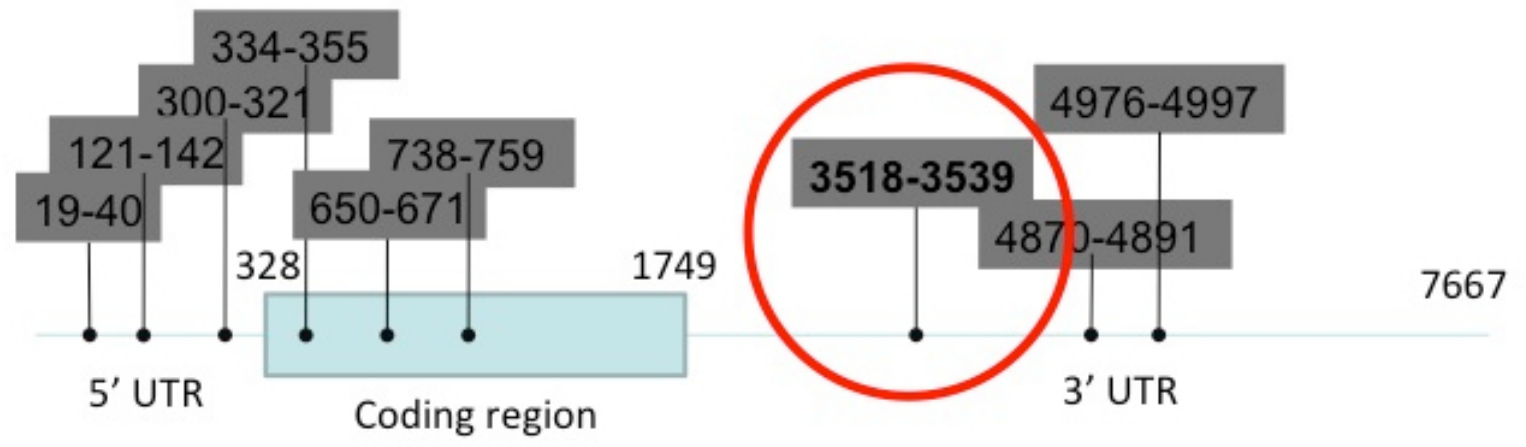

Figure 2-6. Schematic depicting the location of predicted miR-375 binding sites on the MTDH transcript.

Circled location was the only site predicted by all seven in silico algorithms, and was the site cloned into the luciferase expression plasmid. 


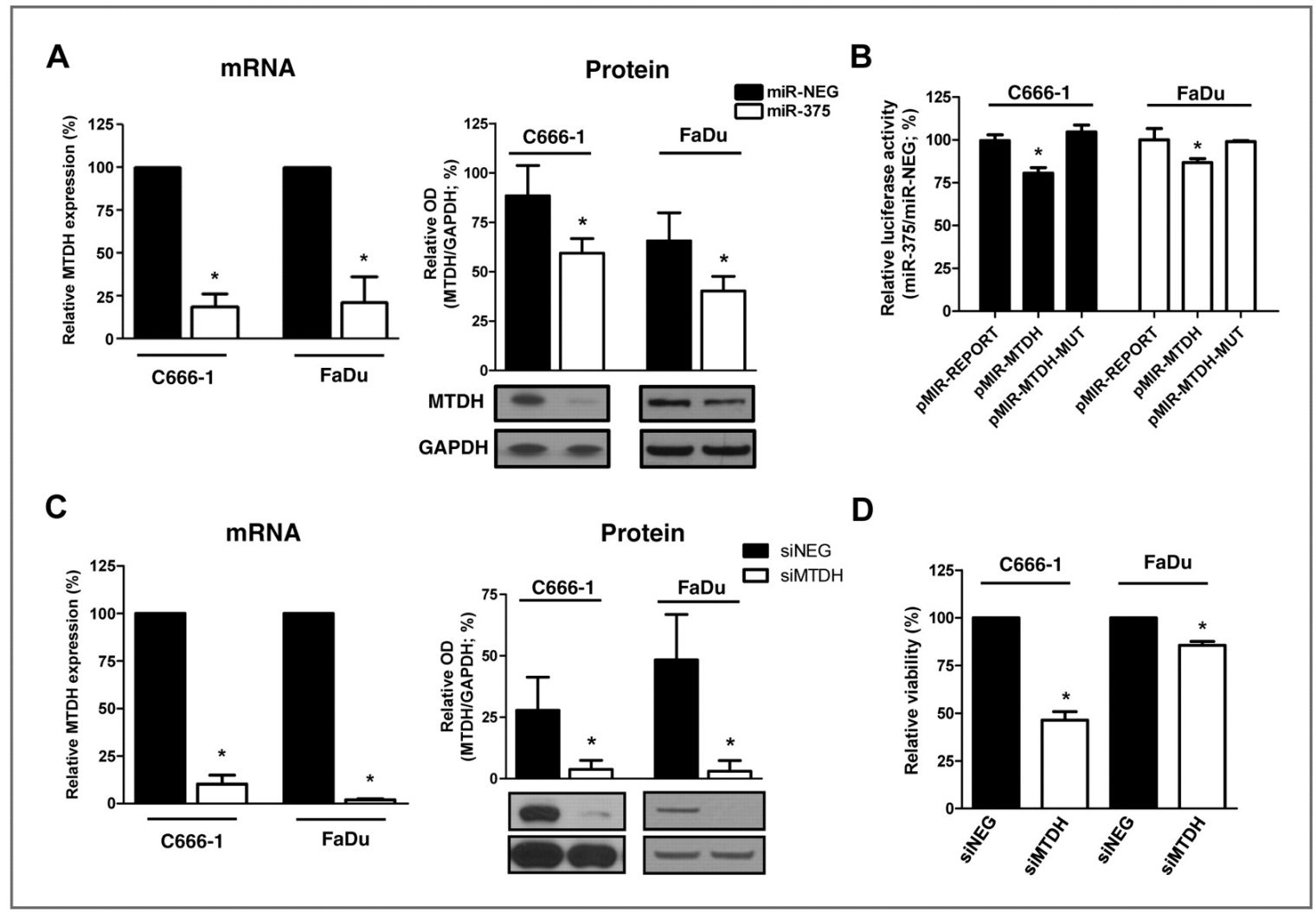

Figure 2-7. Identification of mRNA targets of miR-375

(A) MTDH expression at both the mRNA (left) and protein (right) level after pre-miR-375 or pre-miR-NEG transfection in C666-1 and FaDu cells. (B) Relative luciferase activity in C666-1 or FaDu cells after co-transfection with pMIR-REPORT, pMIR-MTDH or pMIR-MTDH-MUT vectors and pre-miR-375 or pre-miR-NEG. (C) MTDH expression at both the mRNA (left) and protein (right) level after siMTDH or siNEG transfection in C666-1 and FaDu cells. (D) Relative viability in C666-1 and FaDu cells transfected with siMTDH compared to that in cells transfected with the negative control sequence. OD, optical density; *indicates $\mathrm{p}<0.05$ 


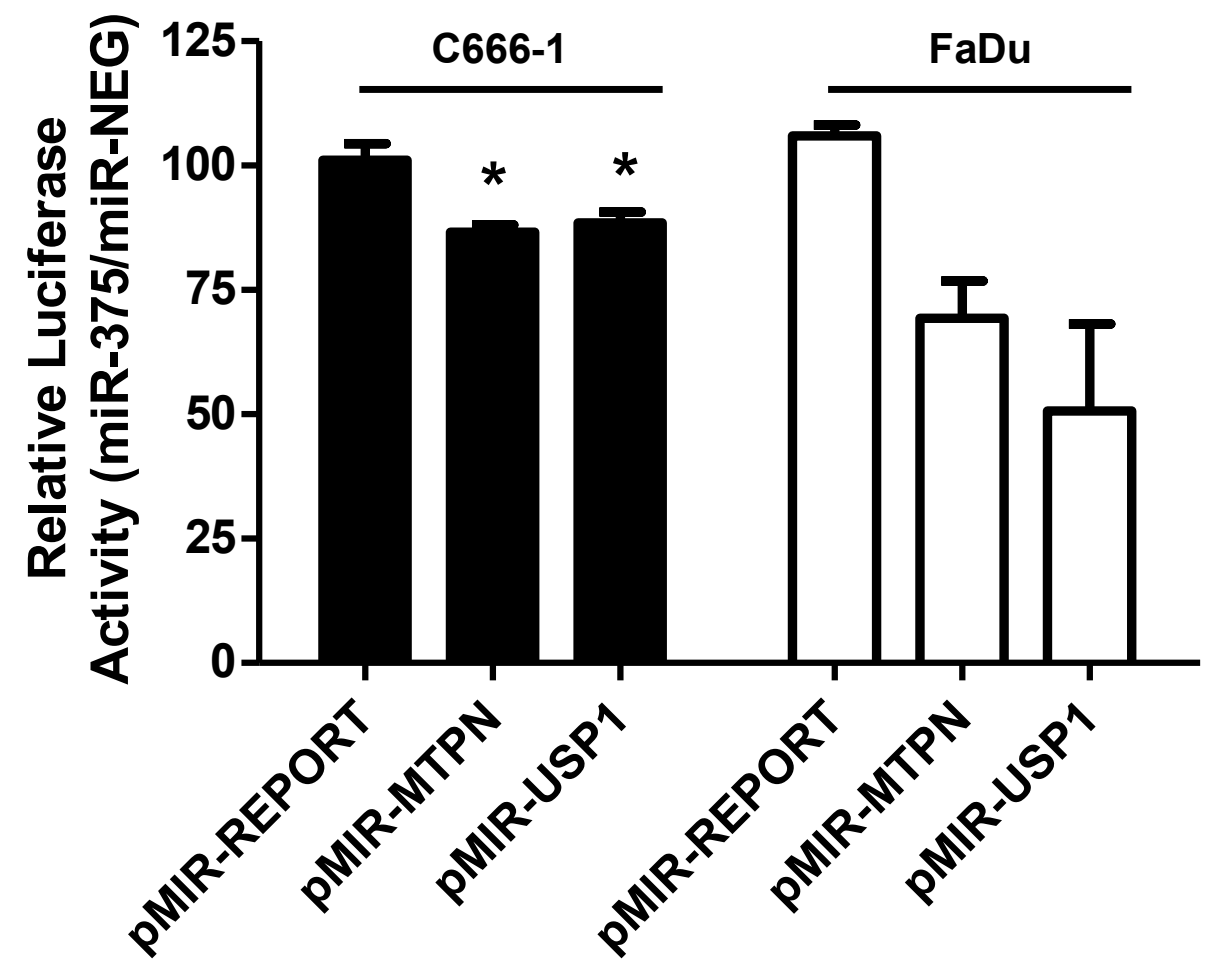

Figure 2-8. Interaction of miR-375 with MTPN and USP1 3' UTRs.

Luciferase assay results illustrating the interaction between miR-375 with its predicted binding sites in the 3'UTRs of the MTPN and USP1 transcripts in both C666-1 and FaDu cells.

$*$ indicates $\mathrm{p}<0.05$ 


\subsubsection{Effects of MTDH knock-down on HNC cells}

The potential oncogenic role of MTDH was investigated by evaluating the phenotypic consequences of transfecting HNC cells with siRNA directed against MTDH (siMTDH). MTDH knock-down by this siRNA sequence was confirmed at both the transcript and protein level (Fig 2-7C). Similar to the effects of pre-miR-375 transfection, siMTDH transfection significantly reduced cell viability in both C666-1 (>50\%), and FaDu ( $20 \%)$ cells (Fig 2-7D). To ensure that the observed cytotoxicity was not due to off-target effects, the MTS experiments were repeated using a second distinct siRNA sequence targeting $\mathrm{MTDH}$, demonstrating a similar effect (Fig 2-9A). As yet another level of corroboration, a rescue plasmid expressing a MTDH transcript refractory to siRNA was co-transfected with siMTDH, which completely abrogated any siMTDH-mediated cytotoxicity, confirming that this was indeed an MTDH knock-down-specific effect (Fig 2-9B).

Metadherin is frequently reported to promote metastasis in human cancers; hence, the effect of miR-375 and MTDH on HNC cell migration and invasion were evaluated using in vitro trans-well migration assays. Compared to their corresponding negative controls, transfection with pre-miR-375 significantly reduced migration of both C666-1 and FaDu cells by $57 \%$ and $80 \%$, respectively (Fig $2-10 \mathrm{~A}$ ). Similar inhibition of migration was observed in cells transfected with siMTDH, with 75\% reduction in C666-1 cells and 86\% in FaDu cells (Fig 2-10A). Moreover, siMTDH and pre-miR-375 transfection also resulted in $86 \%$ and $92 \%$ reduction in invasion of $\mathrm{FaDu}$ cells, respectively (Fig 2-10B). C666-1 cells are unable to penetrate the matrigel coating used in the invasion assay; hence this property could not be assessed in the NPC model. 

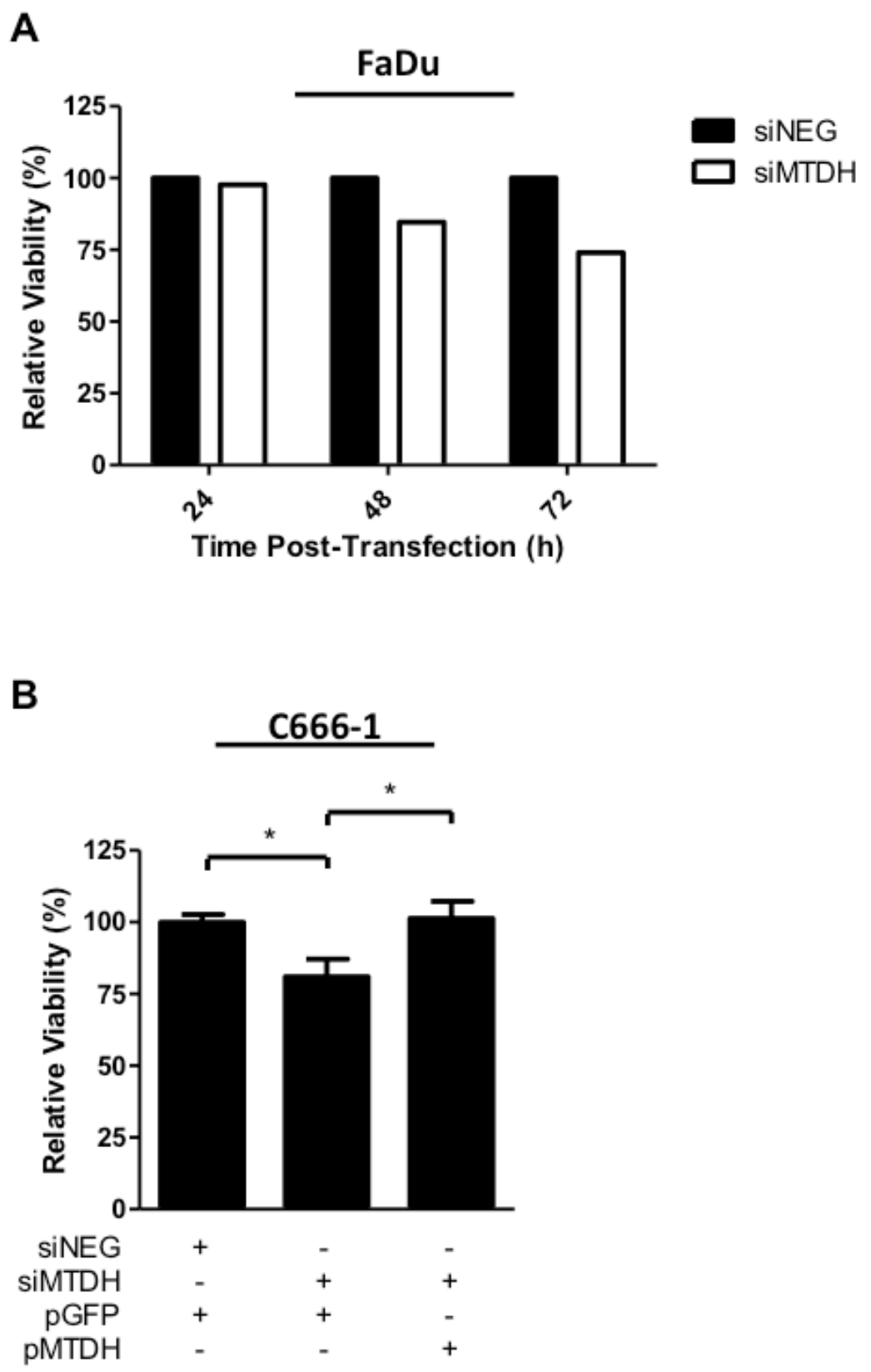

Figure 2-9.Specificity of siRNA mediated cytotoxic effects

(A) The effect of an additional, distinct siRNA sequence targeting MTDH on viability of FaDu cells as measured by the MTS assay. B) Rescue of C666-1 cells from siMTDH-mediated cytotoxicity by co-transfection with a plasmid encoding a si-resistant MTDH transcript (pMTDH). *indicates $\mathrm{p}<0.05$ 
A
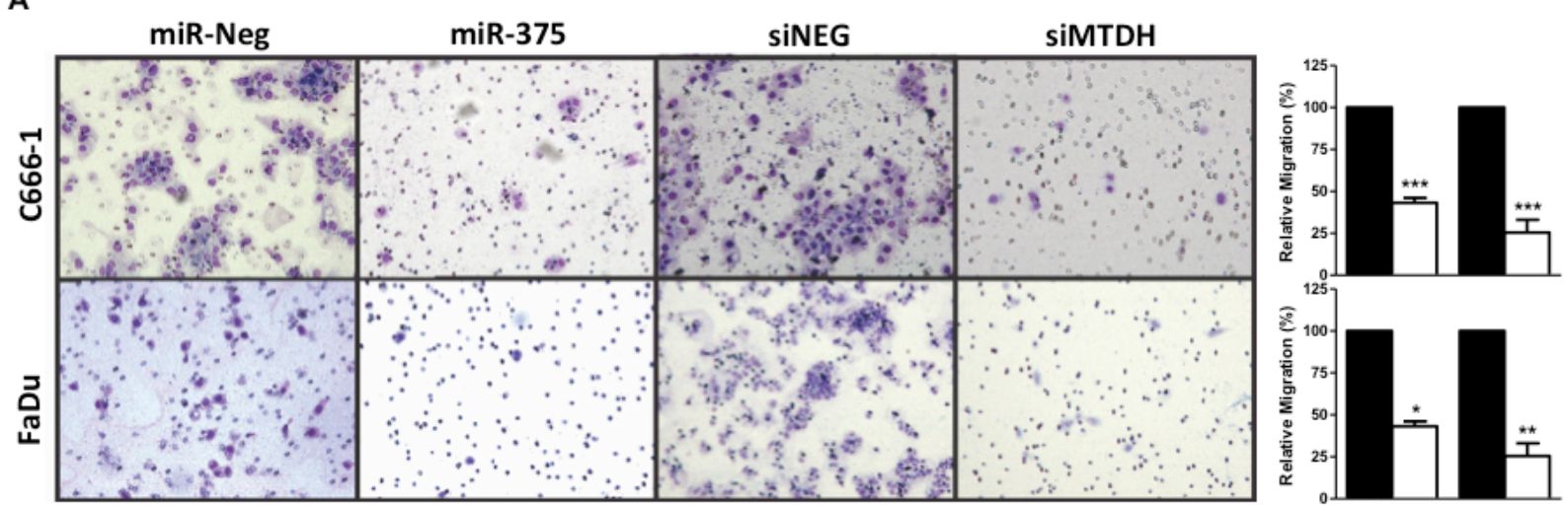

B
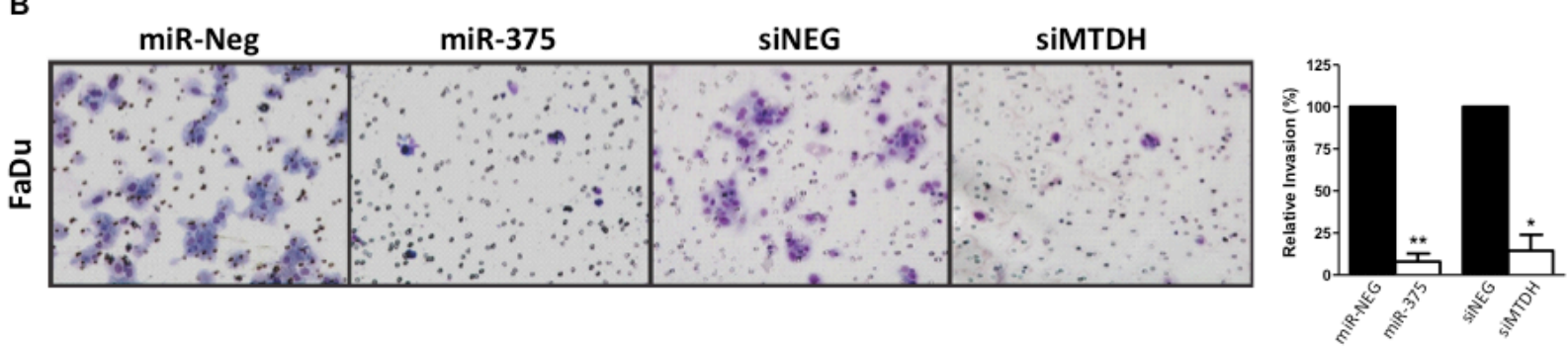

Figure 2-10. Migration and invasion of miR-375 or siMTDH in HNC cells

(A) Representative images (left) and bar graphs (right) depicting the migratory ability of C6661 and $\mathrm{FaDu}$ cells after pre-miR-375 or siMTDH transfection compared to corresponding negative controls. (B) Representative images (left) and bar graphs (right) depicting the invasive ability of FaDu cells after pre-miR-375 or siMTDH transfection compared to corresponding negative controls. *indicates $\mathrm{p}<0.05 ; * *$ indicates $\mathrm{p}<0.01 ; * * *$ indicates $\mathrm{p}<0.001$. 


\subsubsection{Effects of miR-375 transfection and MTDH knock-down on PI(3)K-Akt signalling}

Western blot analyses were performed to investigate the signaling pathways downstream of miR-375 over-expression or MTDH knockdown in FaDu and C666-1 cells. The PI(3)K-Akt pathway has been previously reported to be activated by MTDH over-expression; specifically increased phosphorylation of Akt (p-Akt), although the mechanism by which this induction occurs remains poorly understood ${ }^{272-274}$. Concordantly, a significant reduction in pAkt was observed in both C666-1 and FaDu cells after siMTDH knockdown (Fig 2-11A\&B), phenocopied by miR-375 transfection in FaDu cells (Fig 2-11B), but not so for C666-1 cells (Fig 2-11A). Total Akt expression remained unchanged under these conditions. 
A

C666-1
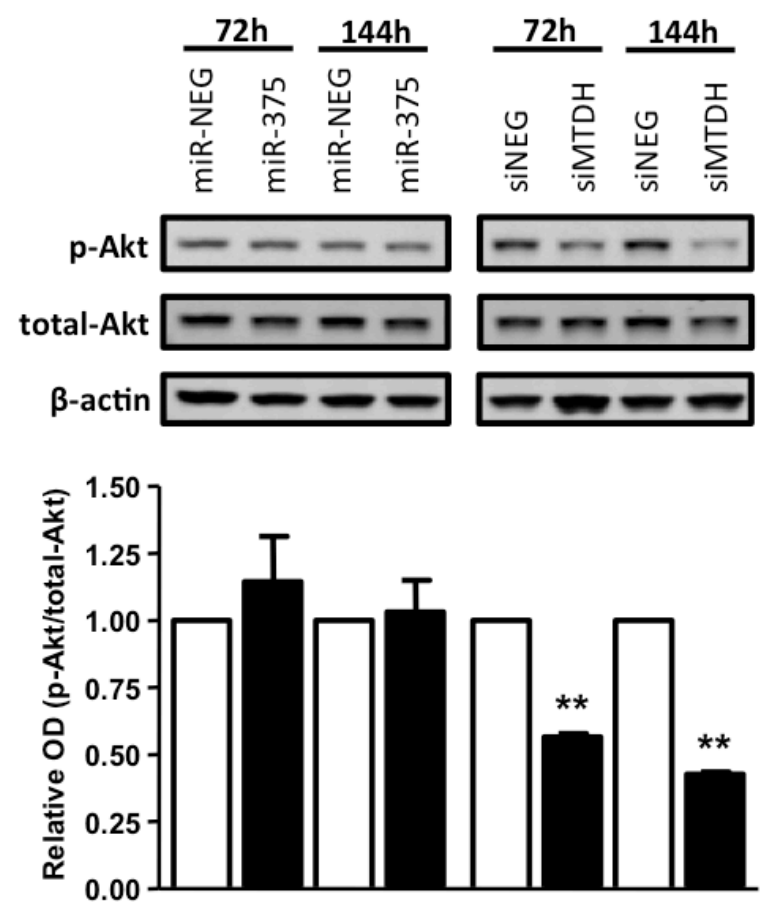

C

C666-1
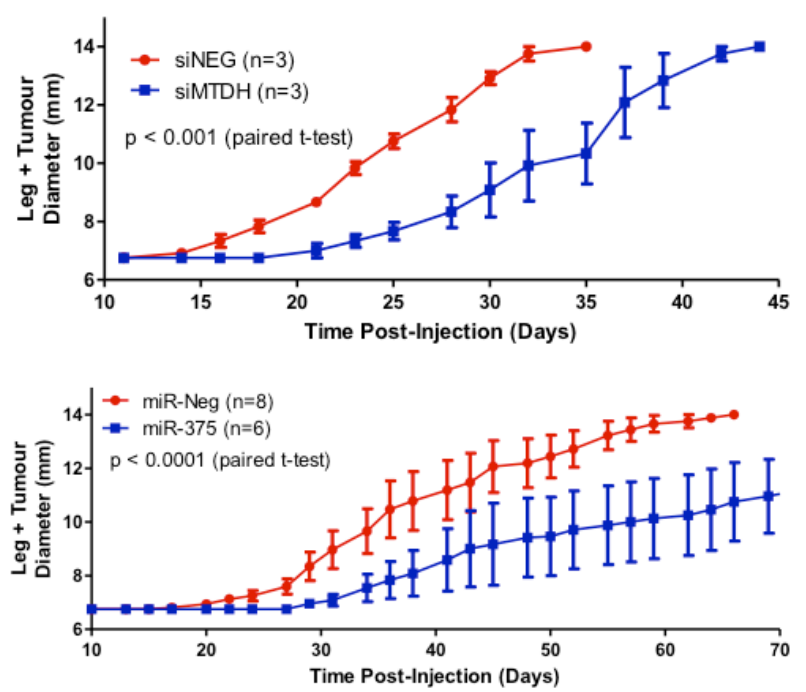

B
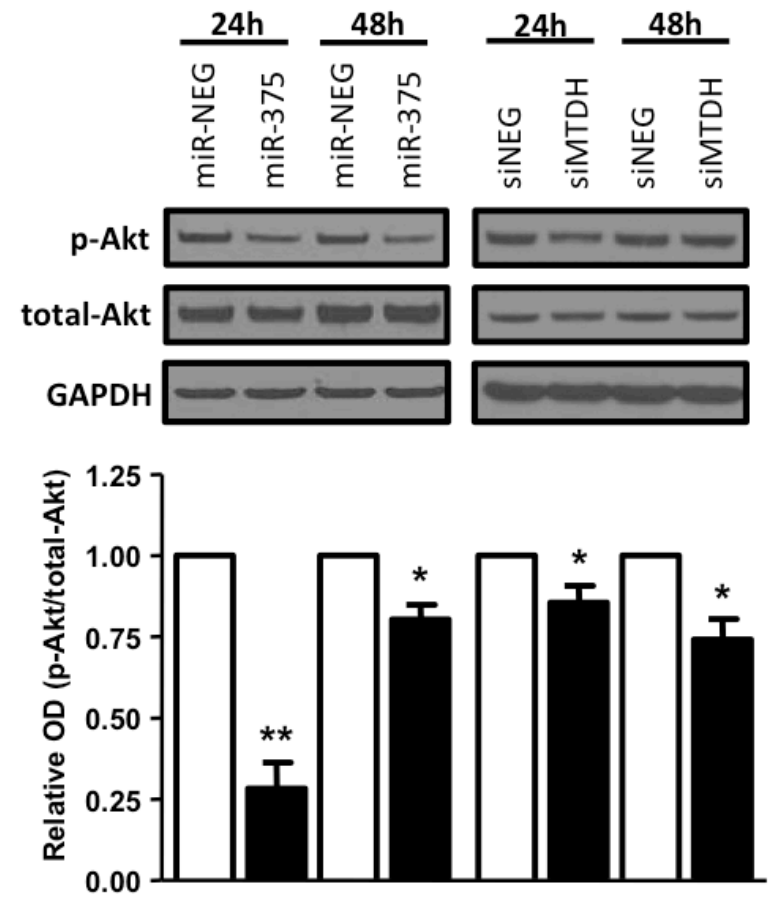

D
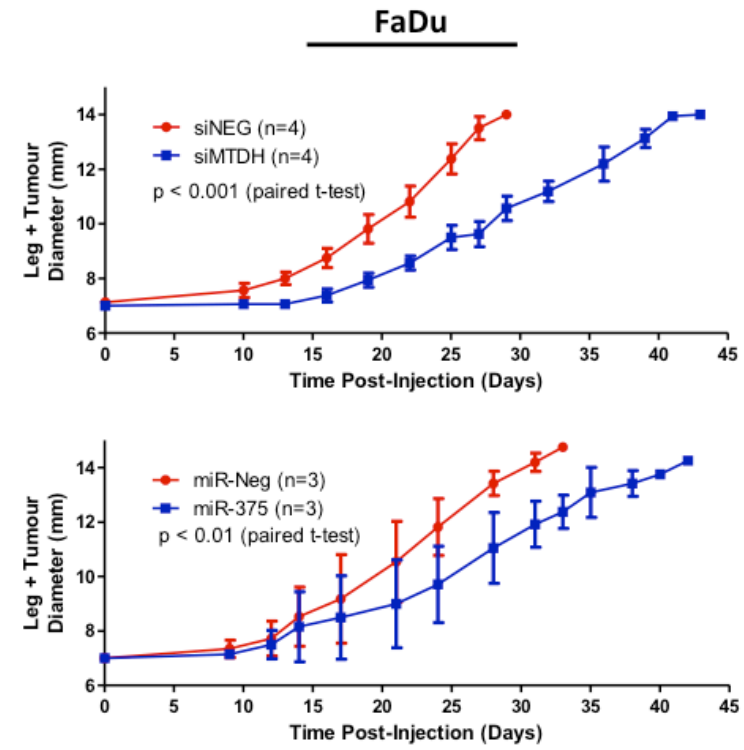

Figure 2-11. Down-stream signaling, and in vivo effects of miR-375 and siMTDH in HNC models.

Representative western blot images and relative quantification of p-Akt protein levels in (A) C666-1 and (B) FaDu cells transfected with pre-miR-375 or siMTDH, compared to corresponding negative controls. (C) \& (D) Tumour-plus-leg diameter measurements of (C) C666-1 and (D) FaDu cell xenografts transfected with siMTDH, pre-miR-375 or corresponding negative controls. OD, optical density; $*$ indicates $\mathrm{p}<0.05$; ** indicates $\mathrm{p}<0.01$. 


\subsubsection{MTDH knock-down and miR-375 transfection delayed tumour formation in- vivo}

To investigate the effects of miR-375 over-expression and MTDH knock-down on head $\&$ neck tumour forming ability, FaDu and C666-1 cells transfected with siMTDH, pre-miR-375 or corresponding negative controls were injected intra-muscularly into SCID mice. The results demonstrated that both miR-375 over-expression and MTDH knock-down resulted in a significant delay in tumour formation wherein such transfected tumours attained the humane endpoint $(\mathrm{TLD}=14 \mathrm{~mm}) \sim 10-15$ days later than tumours transfected with the corresponding negative controls (Fig 2-11C, 2-11D).

\subsubsection{Over-expression of MTDH in primary HNC samples}

The relevance of MTDH in HNC was further investigated in primary human HNC samples in the previously evaluated $20 \mathrm{HNSCC}^{261}$, as well as the same $92 \mathrm{NPC}$ samples for which miR-375 expression has already been examined (Fig 2-3C). Significantly higher MTDH transcript levels were detected in both HNSCC $(\mathrm{p}=0.048)$ and NPC patient samples $(\mathrm{p}<0.0001)$ compared to corresponding normal controls as measured by qRT-PCR (Fig 2-12A \& B). Using a 2-fold cut-off, MTDH mRNA over-expression was detected in 65\% and 93\% of HNSCC and NPC samples, respectively. Over-expression of MTDH at the protein level was corroborated in 94 NPC patient samples (including the 92 previously investigated cases) using immunohistochemistry (IHC), illustrating that MTDH expression was predominantly localized to the cell membrane or cytoplasm, with little to no immuno-staining observed in the surrounding stroma (Fig 2-12C). The vast majority of NPC samples had cytoplasmic MTDH immuno-expression (91 out of 94 cases, or $97 \%$ ), with only one sample showing nuclear 
staining (Fig 2-12D). Strikingly, when dichotomized based on MTDH transcript expression level, NPC patients with high expression ( $\geq$ median) had significantly worse overall $(\mathrm{HR}=2.34$; $p=0.04)$, disease-free $(H R=2.08 ; p=0.04)$, and distant relapse-free $(H R=6.38 ; p=0.005)$ survival compared to patients with low MTDH (<median) expression (Fig 2-12E). The association of MTDH expression with two additional factors that are potentially predictive of distant relapse in NPC patients was also explored; namely treatment, and stage. Firstly, MTDH expression was not different as a function of treatment (chemoradiation vs. radiation alone; Fig 2-13A). Additionally, when the radiation only group of patients was analyzed, MTDH remained a powerful predictor of distant relapse (Fig 2-13B). A similar trend was observed for the chemoradiation patients, but the difference was not statistically significant $(p=0.17)$, likely due to the small sample size (Fig 2-13B). Secondly, MTDH expression was interestingly inversely related to stage, being lower in Stage III/IV vs. Stage I/II patients ( $\mathrm{p}=0.04$; Fig 2-13C). Importantly, however, when patients were further categorized according to both stage (I/II vs. III/IV), as well as MTDH expression (<median $v s . \geq$ median), this was a highly significant association ( $p=0.006$; Fig 2-13D). Specifically, Stage I/II patients with low MTDH expression had $0 \%$ distant relapse; conversely, Stage III/IV patients plus increased MTDH expression had the highest risk of distant relapse (5-year rate of 37\%), suggesting that using both variables could be quite informative in terms of prognosticating for metastatic disease in NPC. Due to the small sample size and mal-distribution of outcome of the HNSCC patients (the majority of the 20 samples had relapsed disease), survival analysis was not performed on this cohort. 
A

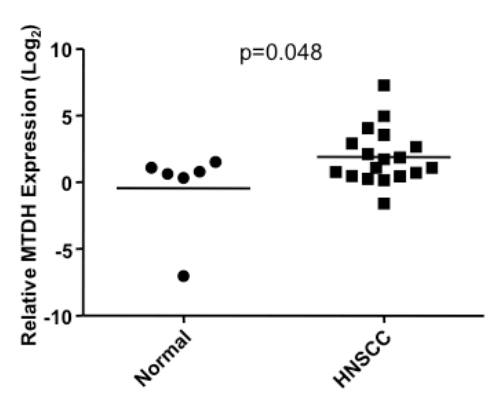

C

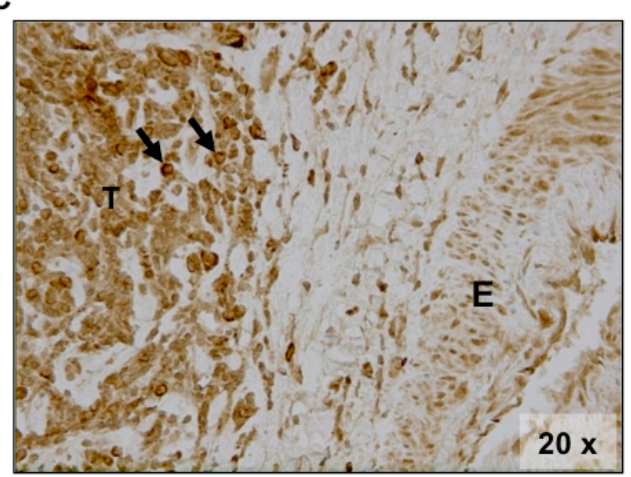

E
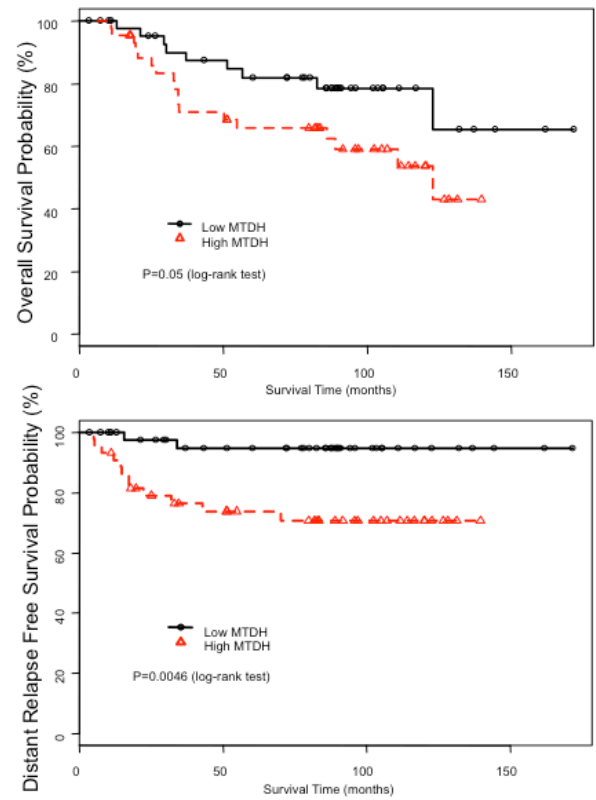

B

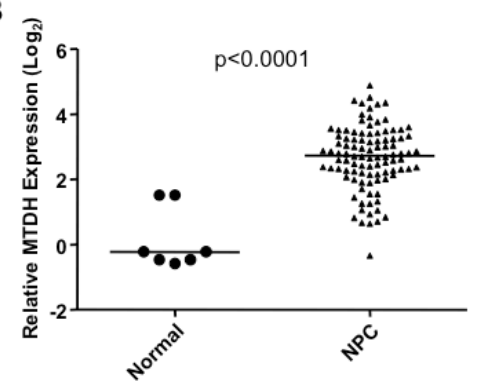

D
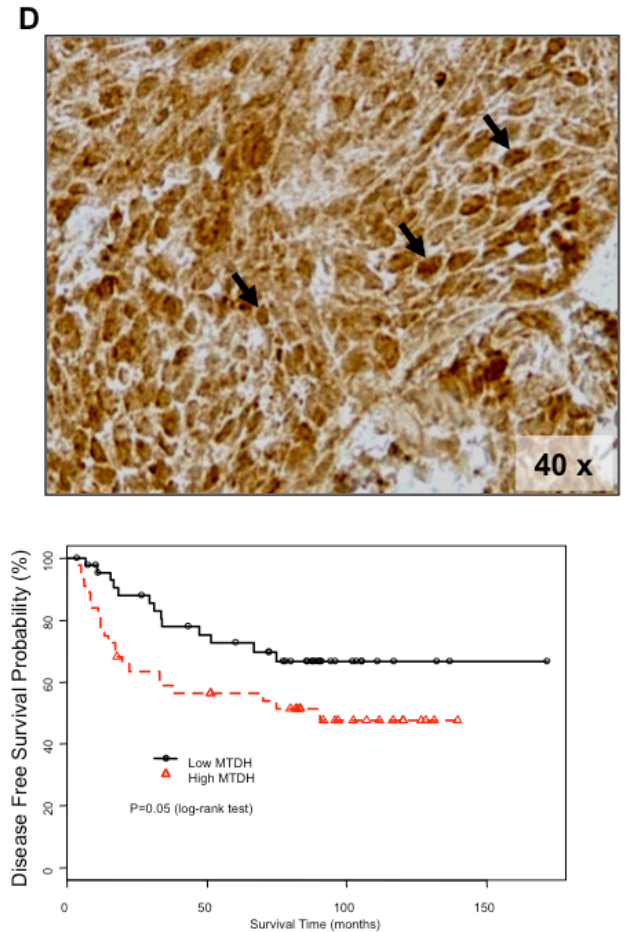

Figure 2-12. MTDH Expression in HNC patient samples.

MTDH expression measured by qRT-PCR in (A) 20 HNSCC and (B) 92 NPC patient biopsy samples, in comparison to 6 normal laryngeal epithelial tissues, and 7 normal nasopharyngeal epithelial tissues, respectively. (C) Representative images of immunohistochemical analysis of MTDH expression in primary NPC biopsy samples. Arrows indicate tumour cells exhibiting (C) cytoplasmic membrane or (D) nuclear expression of MTDH. T, tumor cells; E, endothelial cells. (E) Kaplan-Meier plots of overall survival, disease-free survival, and distant relapse-free survival for NPC patients dichotomized based on high ( $>$ median) vs. low (<median) MTDH transcript expression level. 
A

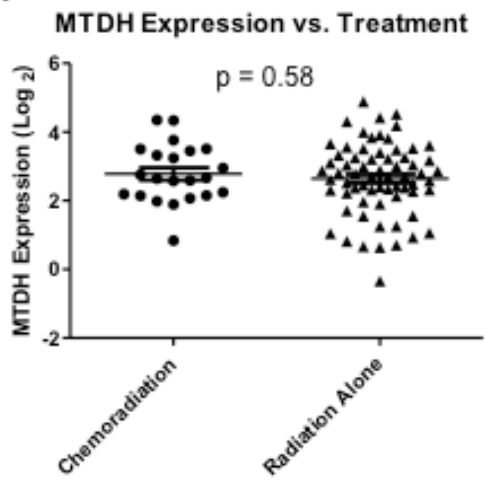

C

MTDH Expression vs. Stage

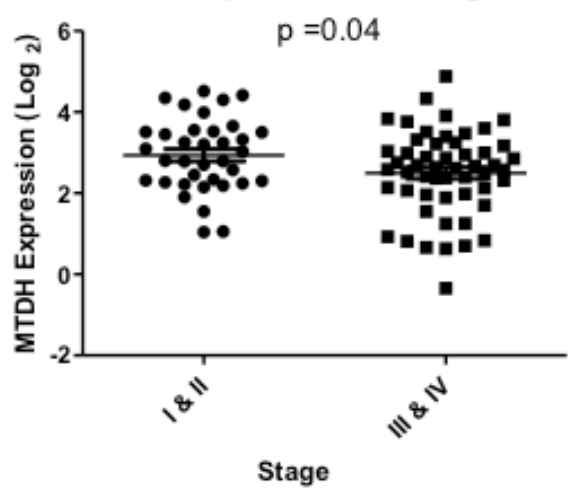

B
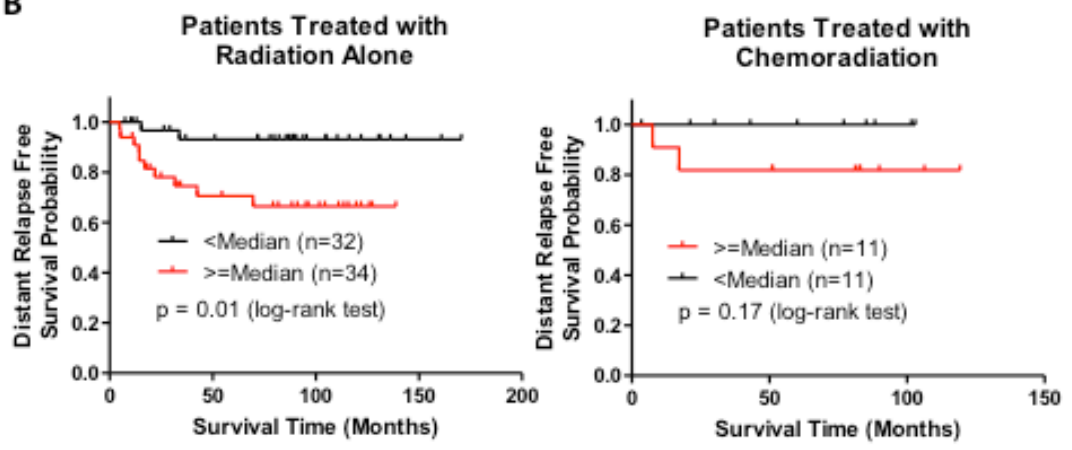

D

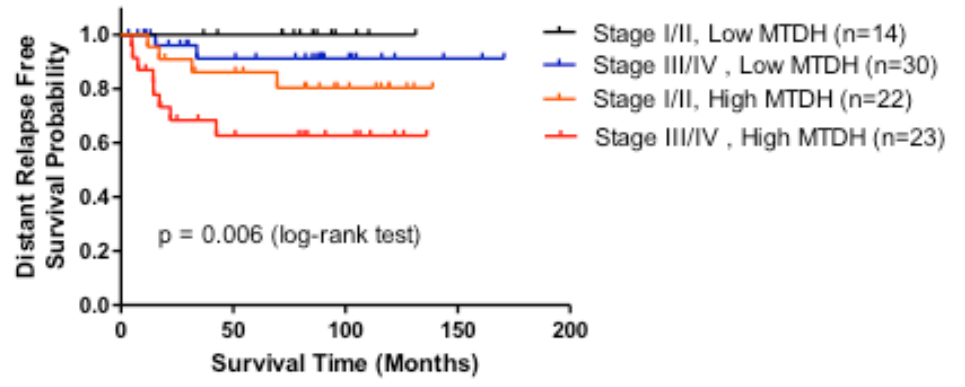

Figure 2-13. Association of MTDH expression with treatment modality and tumour stage. (A) MTDH expression in patients treated with chemoradiation $(n=22)$ vs. radiation alone ( $\mathrm{n}=66)$. (B) Kaplan-Meier plots dichotomized based on MTDH expression level for NPC patients treated with radiation alone (left) or chemoradiation (right). (C) MTDH expression in patients with stage I/II $(n=36)$ vs. stage III/IV $(n=53)$ disease. (D) Kaplan-Meier plot with patients divided into four groups based on stage (I/II or III/IV) and MTDH expression ( $<$ median or $\geq$ median). 


\subsection{Discussion}

Our recent study profiling miRNA expression in locally advanced HNSCC reported a high frequency of miR-375 under-expression in these tumours, and suggested its potential tumor suppressor function ${ }^{261}$. The current report demonstrates that miR-375 is also downregulated in $75 \%$ of NPCs and confirms a tumour suppressor function for this miRNA in HNC cell lines. Furthermore, MTDH has been verified as bona fide target of miR-375 in HNC. During the preparation of this report, MTDH was also identified independently by another group as a target of miR-375 in HNSCC ${ }^{275}$. However, to the best of our knowledge, this study is the first to support a role for the dysregulation of miR-375 and MTDH in promoting metastases in HNCs; particularly in NPC.

MiR-375 was first described in the development and function of pancreatic islet cells $^{276,277}$, and more recently in human embryonic stem cell development ${ }^{278}$. Mounting data however, suggest that miR-375 might also be playing an equally important role in human cancers. Several groups have recently demonstrated a tumour suppressor role for miR$375^{261,271,279,280}$, congruent with our own data in this study. Reduced miR-375 expression has in fact been reported to be associated with worse outcome for patients with esophageal carcinoma ${ }^{281,282}$; high miR-221 to low miR-375 ratio has also been suggested as a potential diagnostic marker for $\mathrm{HNSCC}^{279}$. Conversely, several groups have also described a potential oncogenic role for miR- $375^{283,284}$, underscoring that similar to many other miRNAs, miR-375 can function as both a tumour suppressor and an oncogene, probably dependent upon context and cancer type.

Chromosomal loss and promoter hypermethylation are two common mechanisms for inactivation of tumour suppressor genes. Deletion of chromosome 2q35 has indeed been 
reported in $\mathrm{HNSCC}^{285}$. In our current work, bisulfite sequencing demonstrated that $\mathrm{CpG}$ islands proximal to the miR-375 locus are highly methylated (Fig 2-3D), and exposure to 5'-aza-2deoxycytidine resulted in a significant re-expression of miR-375 (Fig 2-3E), corroborating that hypermethylation is indeed one important mechanism for miR-375 under-expression in $\mathrm{HNC}$, similar to the previous report in $\mathrm{HCC}^{286}$. Hence, chromosomal loss and hypermethylationmediated silencing are two likely mechanisms leading to miR-375 down-regulation in HNC.

In terms of MTDH over-expression in HNC, miR-375 down-regulation is clearly one documented mechanism, but the down regulation of other miRNAs could also play a role in this process. As an example, miR-26a was recently reported to be under-expressed in breast cancer, targeting both MTDH and enhancer of zeste homolog 2 (EZH2) ${ }^{286}$. Our own group has previously reported significant miR-26a down-regulation in primary $\mathrm{HNSCC}^{260}$, as well as $\mathrm{NPC}^{191}$; hence, under-expression of miR-26a might indeed be another mechanism for MTDH over-expression in HNC. A third potential mechanism for MTDH over-expression could be chromosomal amplification; given that 8q22 (genomic location for MTDH) is also frequently amplified in both NPC and HNSCC ${ }^{26,287}$. Indeed, this mechanism for MTDH over-expression has already been reported for both breast ${ }^{262}$ and hepatocellular carcinomas ${ }^{288}$. Taken together, these conglomerate reports illustrate that MTDH over-expression in HNC is likely mediated by a complex interplay between several factors including chromosomal amplification and targeting by multiple miRNAs.

The human $M T D H$ gene was first cloned in astrocytes, where it was shown to be upregulated in response to treatment with TNF- $\alpha$, gp120, or HIV infection; therefore initially denoted as "Astrocyte elevated gene 1" (AEG-1) $)^{261}$. The mouse homologue was cloned shortly thereafter in a murine model for metastatic breast cancer; discovered due to its role in 
selectively homing metastatic breast cancer cells to the lung $^{289}$. Since these seminal observations, MTDH has rapidly emerged as an important mediator in the development and progression for several human cancers ${ }^{262}$. In fact, MTDH over-expression has already been associated with poor outcome for breast ${ }^{262}$, liver ${ }^{288}$, esophageal ${ }^{290}$, renal cell ${ }^{291}$, colorectal $^{292}$, and non-small cell lung cancers ${ }^{293}$, frequently associated with metastases and chemoresistance. However, the molecular mechanisms by which MTDH mediates metastases and/or chemoresistance remain to be clearly elucidated. Some of the suggested downstream signaling events have included activation of PI3K-Akt ${ }^{272}, \mathrm{NF}-\mathrm{kB}^{294}$, MAPK and $\mathrm{Wnt}^{288}$, up-regulation of MMP-9 $9^{295}$ and FOXO3a ${ }^{273,290}$, suppression of FOXO1 ${ }^{274}$, as well as induction of $\mathrm{EMT}^{294}$.

Herein, we report the over-expression of MTDH in both HNSCC and NPC. Due to small sample size $(n=20)$, the significance of MTDH over-expression observed in the HNSCC samples was marginal $(\mathrm{p}=0.048)$, therefore we sought out additional evidence to support these findings. Using a publicly available online (http://www.ebi.ac.uk/gxa/array/U133A) data set mined by Lukk et al. ${ }^{296}$, we identified that MTDH was also significantly over-expressed in their HNSCC samples by 2.1-4.2 fold $(\mathrm{p}<0.08)$. Furthermore, another group has also recently reported the significant over-expression of MTDH in HNSCC using 20 paired HNSCC/Normal samples ${ }^{275}$; hence we are confident regarding MTDH over-expression in primary HNSCC. We also report, for the first time, a role for $\mathrm{MTDH}$ in promoting a metastatic phenotype in $\mathrm{HNC}$, particularly in NPC. The oncogenic function of MTDH was demonstrated both in vitro and in vivo wherein $\mathrm{MTDH}$ affected cell migration and invasion, potentially mediated in part via phosphorylation of Akt. The clinical impact of this observation is extremely important; due to the advent of intensity-modulated radiation therapy (IMRT), the 5-year local control rates for NPC are excellent, at $\sim 90 \%{ }^{13}$. However, the 5 -year OS for NPC remains modest at $\sim 70 \%$ due 
to distant metastases ${ }^{13}$. Hence, therapies that effectively reduce metastases would be of extreme importance in improving survival for NPC patients. As MTDH is primarily expressed at the plasma membrane of breast cancer cells ${ }^{289}$, a DNA vaccine therapy targeting MTDH has been developed, demonstrating some preliminary success in reducing tumour burden and lung metastases in a mouse model of breast cancer ${ }^{297}$. However, such approaches might not be applicable to HNC, given that MTDH expression appears to be predominantly cytoplasmic in this disease (Fig 2-12C).

In conclusion, a novel pathway of miR-375 down-regulation, leading to MTDH overexpression has been documented in human $\mathrm{HNC}$, which has significant clinical implications as a mechanism by which HNC metastases could develop (Figure 2-14). Further unraveling of the MTDH protein structure and function, as well as the molecular mechanisms by which it activates a metastatic cascade could lead to the development of small molecule inhibitors, which can be examined as a potential therapeutic strategy by which outcome can be improved for future patients with HNC. 


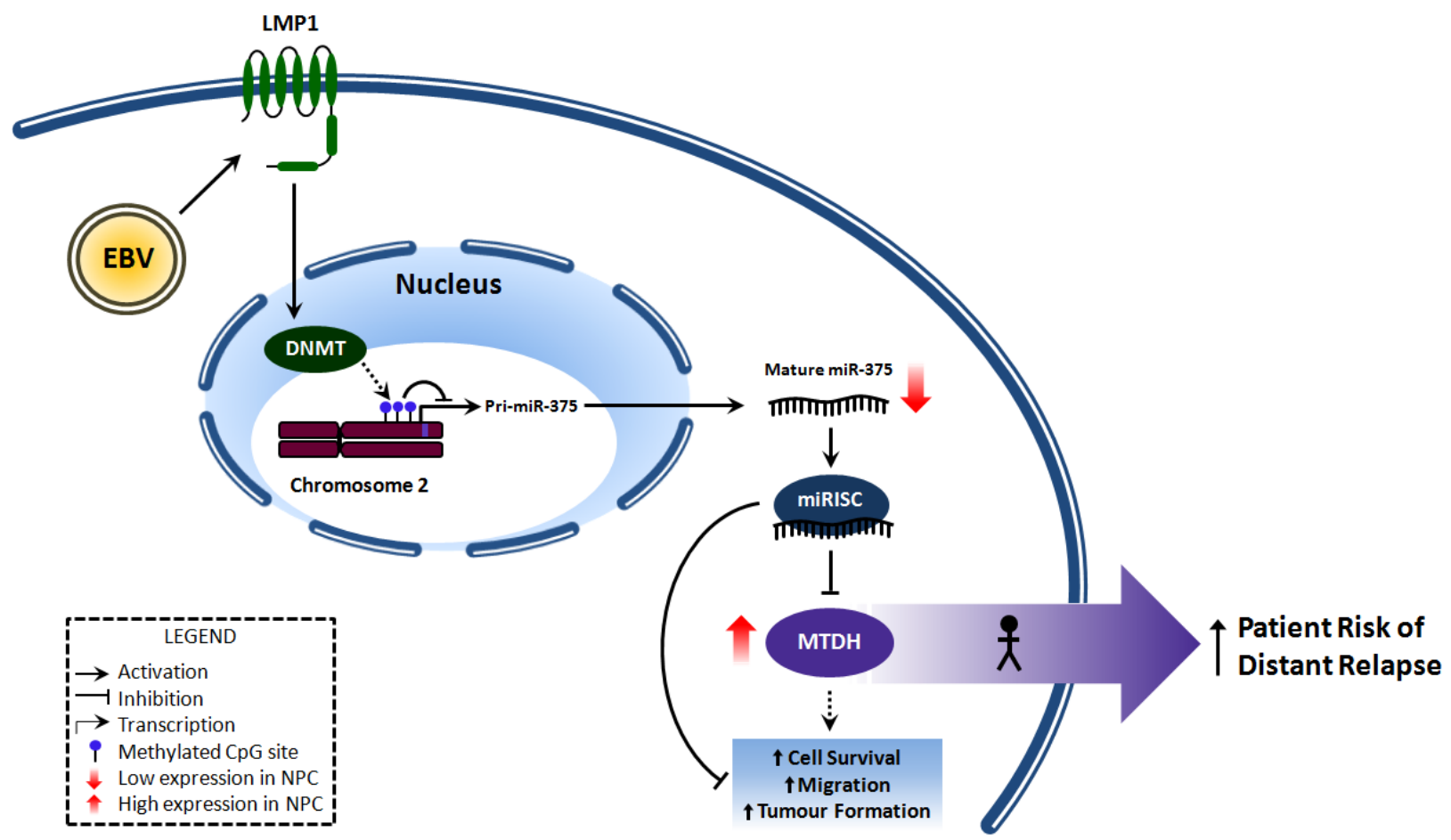

Figure 2-14. Working model detailing the role of the miR-375-MTDH axis in NPC. 


\section{Chapter 3}

\section{The Identification of a microRNA Signature Associated with Risk of Distant Metastasis in Nasopharyngeal Carcinoma}

Jeff P. Bruce, Angela B.Y. Hui, Wei Shi, Bayardo Perez-Ordonez, Ilan Weinreb, Wei Xu, Daryl M. Waggott, Paul C. Boutros, Brian O’Sullivan, John Waldron, Shao Hui Huang, Eric X. Chen, Ralph Gilbert, Fei-Fei Liu 


\section{The Identification of a microRNA Signature Associated with Risk of Distant Metastasis in Nasopharyngeal Carcinoma}

\subsection{Abstract}

\subsubsection{Background}

Despite significant improvement in locoregional control in the contemporary era of nasopharyngeal carcinoma (NPC) treatment, patients still suffer from a significant risk of distant metastasis (DM). Identifying those patients at risk of DM would aid in personalized treatment in the future. MicroRNAs (miRNAs) play many important roles in human cancers; hence, we proceeded to address the primary hypothesis that there is a miRNA expression signature capable of predicting DM for NPC patients.

\subsubsection{Methods}

The expression of 734 miRNAs was measured in 125 (Training Set) and 121 (Validation Set) clinically annotated NPC diagnostic biopsy samples. A signature associated with risk of DM was generated by fitting a penalized Cox Proportion Hazard regression model to the Training data set, and subsequently tested in the Validation set. Pathway enrichment analysis was then performed on validated targets of the miRNAs comprising the final signature.

\subsubsection{Results}

A 4-miRNA expression signature associated with risk of developing DM was identified in a Training set (HR 8.25; $\mathrm{p}<0.001)$ and validated in an independent Validation set (HR 3.2; $\mathrm{p}=0.01$ ), and observed to be the strongest independent predictor when multivariate analysis was performed. Pathway enrichment analysis indicated that the targets of these miRNAs appear to be converging on cell-cycle pathways. 


\subsubsection{Conclusion}

This 4-miRNA signature adds to the prognostic value of the current "gold standard" of TNM staging. In-depth interrogation of these 4-miRNAs will provide important biological insights that could facilitate the discovery and development of novel molecularly targeted therapies to improve outcome for future NPC patients.

\subsection{Introduction}

Nasopharyngeal carcinoma (NPC) has a unique set of etiological, epidemiological and biological characteristics that renders it distinct from other epithelial malignancies of the head and neck region. The primary curative treatment for NPC is radiotherapy (RT) for patients with early stage disease, and concomitant chemoradiotherapy (CRT) for those with locally advanced disease. Advances in RT delivery such as intensity-modulated radiation therapy (IMRT), and increasing use of CRT have led to significantly improved loco-regional control (LRC) for NPC patients, with 5-year LRC rates now well over $90 \%{ }^{298}$. However, distant metastasis (DM) rates have not improved, and late metastatic disease remains the major cause of mortality in this patient population $^{298}$. Currently, TNM staging is the primary tool utilized clinically to prognosticate outcomes for NPC patients. Other indicators such as tumor volume ${ }^{299}$, plasma Epstein-Barr Viral (EBV) DNA titre ${ }^{128,300}$, and levels of expression of various proteins and transcripts ${ }^{157,301,302}$ have been reported to correlate with clinical outcomes; to date however, none has been universally adopted in the clinical management of NPC patients.

MicroRNAs (miRNA) are small non-protein-coding RNA molecules that function to reduce the expression of protein coding genes at the post-transcriptional level. Since their identification in $1993^{303}$, miRNAs have emerged as key regulators of gene expression in nearly all biological processes, including cancer $^{256}$. Additionally, miRNA expression signatures 
associated with prognosis have been identified in numerous malignancies ${ }^{259}$. Hence, we undertook to identify and validate a miRNA expression signature capable of predicting DM for NPC patients.

\subsection{Materials \& Methods}

\subsubsection{Patients and Samples}

Approval for this study was obtained from the Institutional Research Ethics Board (REB) at the Princess Margaret Cancer Center (PM). Diagnostic formalin-fixed paraffinembedded (FFPE) blocks were collected for NPC patients treated at the PM who were diagnosed with NPC between 2000-2009 (Training cohort), and 1993-2000 (Validation cohort) (Table 1). Patients with metastatic disease at the time of diagnosis were excluded. Diagnostic and follow-up data were collected through chart reviews; the clinical characteristics for these two patient cohorts are shown in Table 1. It is important to note that despite the greater utilization of CRT (which reflected clinical practice), and the superior LRC rates in the Training cohort, the 5-year distant metastasis-free survival (DMFS) rates for both cohorts was identical, at $87 \%$.

Normal nasopharyngeal epithelial tissues $(n=17)$ were macro-dissected from FFPE blocks of patients who underwent a quadroscopy and were not diagnosed with NPC. These tissues were examined by at least one head and neck cancer (HNC) pathologist (BP-O or IW), and deemed to be free of malignant cells. 
Table 3-1. Clinical characteristics of patients in the two independent cohorts studied.

\begin{tabular}{|c|c|c|c|}
\hline & $\begin{array}{c}\text { Training Set } \\
\text { 'Dx '00-'09; n=125 }\end{array}$ & $\begin{array}{c}\text { Validation Set } \\
\text { (Dx '93-'00; } n=121 \text { ) }\end{array}$ & p-value* \\
\hline \multicolumn{4}{|l|}{ Age (years) } \\
\hline median & 52 & 48 & \\
\hline \multirow[t]{2}{*}{ range } & $14-89$ & $16-79$ & 0.30 \\
\hline & \multicolumn{2}{|c|}{ Frequency (\%) } & \\
\hline \multicolumn{4}{|l|}{ Gender } \\
\hline Male & $86(67)$ & $56(71)$ & \\
\hline Female & $39(33)$ & $23(29)$ & 0.52 \\
\hline \multicolumn{4}{|l|}{ T stage } \\
\hline T1 & $37(30)$ & 37 (31) & \\
\hline T2 & $20(16)$ & $30(25)$ & \\
\hline T3 & $28(22)$ & $24(20)$ & \\
\hline T4 & $39(31)$ & $28(23)$ & 0.26 \\
\hline Unable to evaluate & $1(<1)$ & $1(<1)$ & \\
\hline \multicolumn{4}{|l|}{ N stage } \\
\hline NO & $25(20)$ & $24(20)$ & \\
\hline N1 & $33(26)$ & $50(41)$ & \\
\hline N2 & $52(42)$ & $38(31)$ & \\
\hline N3 & $15(12)$ & $8(7)$ & 0.05 \\
\hline Unable to evaluate & none & $1(<1)$ & \\
\hline \multicolumn{4}{|l|}{ TNM Stage } \\
\hline I (\%) & $11(8)$ & $10(8)$ & \\
\hline II (\%) & $22(18)$ & $37(31)$ & \\
\hline III (\%) & $41(33)$ & $39(32)$ & \\
\hline IV (\%) & $51(41)$ & $34(28)$ & 0.07 \\
\hline Unable to evaluate & none & $1(<1)$ & \\
\hline \multicolumn{4}{|l|}{ Treatment } \\
\hline Radiation only & $34(27)$ & $86(71)$ & \\
\hline Radiation + chemo & $91(73)$ & 35 (29) & $1.43 \mathrm{E}-11$ \\
\hline
\end{tabular}

5-year Survival \%

$\begin{array}{lccc}\text { Survival } & & & \\ \text { Overall } & 83 \% & 73 \% & 0.22 \\ \text { Disease-Free } & 72 \% & 66 \% & 0.35 \\ \text { Local Relapse-Free } & 90 \% & 76 \% & 0.002 \\ \text { Nodal Relapse-Free } & 94 \% & 88 \% & 0.08 \\ \text { Distant Relapse-Free } & 87 \% & 87 \% & 0.89\end{array}$

*Statistical tests: Wilcoxon rank-sum test (Age), Chi-squared test (Gender, T/N/TNM-stage, Treatment), log-rank test (all survival endpoints). 


\subsubsection{MiRNA Expression Profiling}

A representative section from each block was stained with hematoxylin and eosin (H\&E) and reviewed by a HNC pathologist (BP-O) to identify regions containing malignant cells. Samples were then macro-dissected to ensure that $>70 \%$ of the material analyzed was malignant epithelium. Specimens were de-paraffinized with xylene, and total RNA was extracted using the Recover All Total Nucleic Acid Isolation Kit for FFPE (Ambion, Inc.), according to the manufacturer's instructions. RNA was quantified and purity-assessed using a Nanodrop spectrophotometer (Thermo Scientific). Two hundred nanograms of total RNA from each sample was analyzed according to the manufacturer's instructions using the nCounter ${ }^{\circledR}$ Human miRNA Assay from Nanostring (v1.0) to measure 734 unique human and viral miRNAs. Data were normalized using the NanoStringNorm ${ }^{304}$ (v1.1.13) package in the ' $R$ ' statistical computing environment (v2.15.2). Raw counts were background-corrected by subtracting the mean +2 standard deviations of the negative control probes included in the assay, followed by variance stabilization and normalization $\left(\mathrm{vsn}^{305}\right)$ called through the NanoStringNorm package.

\subsubsection{Statistical Analysis}

All statistical tests were performed in the ' $R$ ' statistical computing environment (v2.15.2), with the exception of pathway enrichment analysis. MiRNA expression in tumour vs. normal samples was compared using Welch's t-tests with the Benjamini \& Hochberg false discovery rate (FDR) correction for multiple comparisons ${ }^{306}$. Prior to all regression analysis, the data were standardized (mean centered and standard deviation scaled), using the R package 'ffmanova'307, to maintain consistency across datasets. In order to identify a signature 
associated with risk of DM, an L1 penalized (Lasso ${ }^{308}$ ) Cox proportional hazard (CoxPH) regression model was fitted to the Training cohort data using the penalized (v0.9-42) package ${ }^{309}$ in R. Leave-one-out cross validation was used to determine the L1 tuning parameter that yielded the highest log-likelihood, and a penalized CoxPH regression model was fitted using this optimal value. After fitting, the 4 miRNAs most strongly associated with DM were selected for inclusion in the risk score (RS) equation in order to maintain the number of events per variable $(E V P)$ at $\sim$ five $^{310}$. The final equation was generated using the regression coefficients from the CoxPH model. RS was calculated for each patient in the Training cohort, and patients were dichotomized to "high risk" (RS $\geq$ median) or "low risk" (RS $<$ median) groups. The difference in DMFS between the two groups was compared using the log-rank test. RS and risk-groups were determined similarly for the Validation cohort, with the cut-point maintained as the median RS in the Training cohort and DMFS compared using the log-rank test. Multivariate analysis was performed by including the 4-miRNA signature risk-group assignment ("high risk" or "low risk") for each patient adjusted with clinical variables (T-Stage, $\mathrm{N}-$ Stage, gender, age, treatment) in a CoxPH model fitted to the combined Training and Validation datasets $(n=242$; one patient removed due to unevaluable nodal stage, three patients removed due to unevaluable tumour stage). The two variables found to be significantly associated with DM in this model (the 4-miRNA signature RS, and N-stage) were then combined to create five risk groups: 1. N0; 2. N1/2 \& Low-RS; 3. N1/2 \& High-RS; 4. N3 \& Low-RS; and 5. N3 \& High-RS. The area-under the curve (AUC) was calculated for the receiver operating characteristic (ROC) curves across multiple time points using the 'survivalROC' package (v1.0.3) $)^{311}$ and the c-statistics for various predictive models were calculated using the 'survConcordance' function in the 'survival' package (v2.37-4) ${ }^{312}$ for R. 


\subsubsection{TCGA Data Retrieval and Analysis}

Level 3 (Reads-per-million; RPM) miRNA-Seq and Level 1 clinical data were downloaded from the Broad Institute Firehose (stddata run 2013_11_14) repository for data generated by TCGA. At the time of analysis, there were $260 \mathrm{HNSCC}$ samples with miRNAseq values and sufficient follow-up data to determine disease specific survival status. RPM data were $\log _{2}$ transformed and standardized (mean centered, standard deviation scaled) before risk scores were calculated using the 4-miRNA equation described above. Patients were dichotomized into "low risk" ( $<$ median RS) vs. "high risk" ( $\geq$ median RS) groups, and then compared using the log-rank test.

\subsubsection{Target Identification and Pathway Analysis}

Validated targets of the 4 miRNAs in the signature were downloaded from the miRWalk database which uses a text-mining algorithm to extract validated miRNA-target relationships from the literature ${ }^{313}$. The union of the 4 lists of targets was inputted into the Database for Annotation, Visualization and Integrated Discovery (DAVID; v6.7) (14 $^{314}$ for pathway enrichment analysis. To ensure that the enrichment observed was not due to bias in the validated target database, a complete list of validated targets for all human miRNAs was downloaded from the miRWalk database, and used as the background for enrichment analysis.

\subsection{Results}

\subsubsection{Differentially Expressed miRNAs}

Amongst the 734 human and viral miRNAs included in the nCounter human miRNA assay, 531 and 442 miRNAs were expressed above background levels in at least one sample in 
the Training and Validation cohorts, respectively. Tumour vs. normal tissue comparisons in the Training (tumour $\mathrm{n}=125$; normal $\mathrm{n}=7$ ), and Validation (tumour $\mathrm{n}=121$; normal $\mathrm{n}=10$ ) data sets yielded 124 and 192 differentially-expressed miRNAs, respectively, at a false discovery rate (FDR) cut-off of 0.05 . Amongst these differentially expressed miRNAs, 81 overlapped between the two data sets, including 30 EBV-encoded miRNAs from the BamHI-A rightward transcripts (BART) region, plus several miRNAs that have been previously reported as differentially expressed in NPC or other cancers (Table 3-2) ${ }^{315-317}$. 
Table 3-2. Differential expression analysis statistics for 81 miRNAs found to be significantly altered in both Training and Validation cohorts.

\begin{tabular}{|c|c|c|c|c|c|c|c|c|}
\hline & \multicolumn{4}{|c|}{ Training } & \multicolumn{4}{|c|}{ Validation } \\
\hline & $\log _{2} \mathrm{FC}$ & Mean & Raw p & Adjusted $\mathrm{p}$ & $\log _{2} \mathrm{FC}$ & Mean & Raw p & Adjusted $\mathrm{p}$ \\
\hline \multicolumn{9}{|l|}{ Human } \\
\hline miR-1978 & 7.33 & 4.70 & $3.04 \mathrm{E}-50$ & $1.68 \mathrm{E}-48$ & 5.15 & 5.35 & $1.08 \mathrm{E}-02$ & $4.54 \mathrm{E}-02$ \\
\hline miR-708 & 7.08 & 4.54 & $3.08 \mathrm{E}-32$ & $8.52 \mathrm{E}-31$ & 1.97 & 8.72 & $1.03 \mathrm{E}-03$ & $6.80 \mathrm{E}-03$ \\
\hline $\mathrm{miR}-301 \mathrm{~b}$ & 4.22 & 2.76 & $1.36 \mathrm{E}-24$ & $3.00 \mathrm{E}-23$ & 1.62 & 1.08 & $2.82 \mathrm{E}-11$ & $5.03 \mathrm{E}-10$ \\
\hline miR-663b & 3.18 & 2.04 & $2.95 \mathrm{E}-19$ & $5.42 \mathrm{E}-18$ & 1.55 & 1.10 & $3.22 \mathrm{E}-09$ & $4.63 \mathrm{E}-08$ \\
\hline miR-1977 & 3.04 & 1.95 & $3.61 \mathrm{E}-19$ & $6.39 \mathrm{E}-18$ & 4.62 & 4.22 & $4.50 \mathrm{E}-03$ & $2.24 \mathrm{E}-02$ \\
\hline miR-130b & 2.22 & 1.44 & $1.70 \mathrm{E}-15$ & $2.50 \mathrm{E}-14$ & 4.74 & 4.77 & 6.79E-03 & $3.19 \mathrm{E}-02$ \\
\hline miR-1253 & 2.97 & 1.90 & $2.19 \mathrm{E}-13$ & $2.85 \mathrm{E}-12$ & 2.10 & 1.80 & $3.42 \mathrm{E}-04$ & $2.60 \mathrm{E}-03$ \\
\hline $\operatorname{miR}-1290$ & 8.90 & 6.00 & $2.71 \mathrm{E}-12$ & $3.32 \mathrm{E}-11$ & 6.00 & 4.25 & $3.03 \mathrm{E}-11$ & $5.23 \mathrm{E}-10$ \\
\hline miR-421 & 3.88 & 2.70 & $1.44 \mathrm{E}-10$ & $1.67 \mathrm{E}-09$ & 3.81 & 3.92 & $3.03 \mathrm{E}-03$ & $1.60 \mathrm{E}-02$ \\
\hline miR-452 & 1.44 & 0.92 & $2.24 \mathrm{E}-09$ & $2.24 \mathrm{E}-08$ & 3.02 & 2.49 & $5.15 \mathrm{E}-03$ & $2.49 \mathrm{E}-02$ \\
\hline miR-224 & 1.24 & 0.80 & $2.28 \mathrm{E}-09$ & 2.24E-08 & 0.63 & 0.40 & $2.16 \mathrm{E}-04$ & $1.74 \mathrm{E}-03$ \\
\hline miR-566 & 1.74 & 1.22 & $1.89 \mathrm{E}-08$ & $1.71 \mathrm{E}-07$ & 1.60 & 1.02 & $1.06 \mathrm{E}-08$ & $1.49 \mathrm{E}-07$ \\
\hline miR-1308 & 12.02 & 8.49 & $3.78 \mathrm{E}-08$ & $3.34 \mathrm{E}-07$ & 2.56 & 10.72 & $1.77 \mathrm{E}-07$ & $2.29 \mathrm{E}-06$ \\
\hline miR-495 & 0.95 & 0.61 & $8.15 \mathrm{E}-08$ & $6.67 \mathrm{E}-07$ & 1.74 & 1.56 & $7.45 \mathrm{E}-03$ & $3.44 \mathrm{E}-02$ \\
\hline miR-1246 & 10.85 & 8.12 & $4.07 \mathrm{E}-07$ & $3.16 \mathrm{E}-06$ & 2.78 & 9.73 & $2.36 \mathrm{E}-04$ & $1.88 \mathrm{E}-03$ \\
\hline miR-369-3p & 0.66 & 0.42 & $1.34 \mathrm{E}-06$ & $9.69 \mathrm{E}-06$ & 0.43 & 0.27 & $1.89 \mathrm{E}-03$ & $1.09 \mathrm{E}-02$ \\
\hline $\operatorname{miR}-2117$ & 7.05 & 5.42 & $2.05 \mathrm{E}-06$ & $1.43 \mathrm{E}-05$ & 3.81 & 3.07 & 4.62E-05 & $4.50 \mathrm{E}-04$ \\
\hline $\operatorname{miR}-885-5 p$ & 0.85 & 0.54 & $1.36 \mathrm{E}-05$ & $8.35 \mathrm{E}-05$ & 1.11 & 0.90 & $1.58 \mathrm{E}-03$ & $9.72 \mathrm{E}-03$ \\
\hline $\operatorname{miR}-362-3 p$ & 0.76 & 0.49 & $1.74 \mathrm{E}-05$ & $1.04 \mathrm{E}-04$ & 3.22 & 2.53 & $1.16 \mathrm{E}-03$ & $7.53 \mathrm{E}-03$ \\
\hline miR-548f & 0.63 & 0.41 & $2.02 \mathrm{E}-05$ & $1.18 \mathrm{E}-04$ & 0.44 & 0.28 & $1.53 \mathrm{E}-04$ & $1.36 \mathrm{E}-03$ \\
\hline miR-653 & 0.88 & 0.57 & $2.40 \mathrm{E}-05$ & $1.36 \mathrm{E}-04$ & 0.50 & 0.34 & $2.49 \mathrm{E}-04$ & $1.95 \mathrm{E}-03$ \\
\hline $\operatorname{miR}-145$ & -5.64 & 6.10 & $4.80 \mathrm{E}-05$ & $2.52 \mathrm{E}-04$ & -2.64 & 9.19 & $1.66 \mathrm{E}-03$ & $1.01 \mathrm{E}-02$ \\
\hline $\operatorname{miR}-125 b$ & -2.64 & 9.25 & $5.71 \mathrm{E}-05$ & $2.97 \mathrm{E}-04$ & -1.18 & 11.69 & $9.94 \mathrm{E}-03$ & $4.33 \mathrm{E}-02$ \\
\hline $\operatorname{miR}-455-5 p$ & 0.63 & 0.40 & $3.12 \mathrm{E}-04$ & $1.41 \mathrm{E}-03$ & 4.43 & 3.49 & $2.32 \mathrm{E}-03$ & $1.29 \mathrm{E}-02$ \\
\hline $\operatorname{miR}-551 b$ & 0.54 & 0.35 & $4.19 \mathrm{E}-04$ & $1.82 \mathrm{E}-03$ & 1.48 & 0.98 & $3.40 \mathrm{E}-07$ & $4.18 \mathrm{E}-06$ \\
\hline miR-324-3p & 0.36 & 0.23 & $9.84 \mathrm{E}-04$ & $3.92 \mathrm{E}-03$ & 2.75 & 2.18 & $1.20 \mathrm{E}-03$ & $7.59 \mathrm{E}-03$ \\
\hline $\operatorname{miR}-21$ & 3.81 & 10.46 & $1.48 \mathrm{E}-03$ & $5.55 \mathrm{E}-03$ & 3.77 & 13.03 & $4.42 \mathrm{E}-04$ & $3.31 \mathrm{E}-03$ \\
\hline miR-320a & 5.69 & 5.78 & $1.50 \mathrm{E}-03$ & $5.56 \mathrm{E}-03$ & 5.68 & 6.30 & $9.14 \mathrm{E}-03$ & 4.11E-02 \\
\hline $\operatorname{miR}-196 b$ & 0.42 & 0.27 & $1.58 \mathrm{E}-03$ & $5.82 \mathrm{E}-03$ & 5.55 & 3.59 & $6.35 \mathrm{E}-32$ & $2.34 \mathrm{E}-30$ \\
\hline miR-199b-5p & -5.64 & 2.91 & $1.63 \mathrm{E}-03$ & $5.91 \mathrm{E}-03$ & -2.47 & 6.39 & $8.54 \mathrm{E}-04$ & $5.97 \mathrm{E}-03$ \\
\hline miR-494 & 8.40 & 13.18 & $1.68 \mathrm{E}-03$ & $6.03 \mathrm{E}-03$ & 4.65 & 13.19 & $7.41 \mathrm{E}-06$ & $8.15 \mathrm{E}-05$ \\
\hline miR-630 & 6.77 & 9.56 & $1.95 \mathrm{E}-03$ & $6.94 \mathrm{E}-03$ & 3.44 & 8.97 & $1.03 \mathrm{E}-02$ & $4.44 \mathrm{E}-02$ \\
\hline miR-1274a & 1.61 & 13.10 & $2.10 \mathrm{E}-03$ & $7.21 \mathrm{E}-03$ & 1.50 & 13.33 & $2.22 \mathrm{E}-03$ & $1.26 \mathrm{E}-02$ \\
\hline miR-874 & 0.37 & 0.24 & $2.20 \mathrm{E}-03$ & $7.47 \mathrm{E}-03$ & 0.51 & 0.35 & $1.07 \mathrm{E}-04$ & $9.68 \mathrm{E}-04$ \\
\hline miR-493 & 4.74 & 6.13 & $2.27 \mathrm{E}-03$ & $7.67 \mathrm{E}-03$ & 5.14 & 6.40 & $4.49 \mathrm{E}-03$ & $2.24 \mathrm{E}-02$ \\
\hline miR-508-3p & 0.60 & 0.39 & $2.82 \mathrm{E}-03$ & $9.24 \mathrm{E}-03$ & 0.31 & 0.22 & $1.05 \mathrm{E}-02$ & $4.49 \mathrm{E}-02$ \\
\hline miR-762 & 0.26 & 0.17 & $3.68 \mathrm{E}-03$ & $1.13 \mathrm{E}-02$ & 0.36 & 0.22 & $2.64 \mathrm{E}-03$ & $1.42 \mathrm{E}-02$ \\
\hline $\operatorname{miR}-18 b$ & 0.29 & 0.18 & $3.75 \mathrm{E}-03$ & $1.14 \mathrm{E}-02$ & 1.61 & 1.02 & $2.25 \mathrm{E}-09$ & $3.33 \mathrm{E}-08$ \\
\hline $\operatorname{miR}-572$ & 0.25 & 0.16 & $4.00 \mathrm{E}-03$ & $1.21 \mathrm{E}-02$ & 0.15 & 0.11 & $1.17 \mathrm{E}-02$ & $4.87 \mathrm{E}-02$ \\
\hline miR-208a & 0.26 & 0.17 & $5.41 \mathrm{E}-03$ & $1.59 \mathrm{E}-02$ & 0.79 & 0.53 & $9.20 \mathrm{E}-07$ & $1.06 \mathrm{E}-05$ \\
\hline $\operatorname{miR}-25$ & 4.27 & 8.25 & $5.62 \mathrm{E}-03$ & $1.65 \mathrm{E}-02$ & 2.25 & 11.10 & $1.90 \mathrm{E}-03$ & $1.09 \mathrm{E}-02$ \\
\hline
\end{tabular}




\begin{tabular}{|c|c|c|c|c|c|c|c|c|}
\hline & $\log _{2} \mathrm{FC}$ & Mean & Raw p & Adjusted $\mathrm{p}$ & $\log _{2} \mathrm{FC}$ & Mean & Raw p & Adjusted $\mathrm{p}$ \\
\hline miR-184 & 0.33 & 0.22 & $7.64 \mathrm{E}-03$ & $2.15 \mathrm{E}-02$ & 3.74 & 2.43 & $8.56 \mathrm{E}-16$ & $2.21 \mathrm{E}-14$ \\
\hline miR-96 & 4.48 & 5.83 & $7.70 \mathrm{E}-03$ & $2.15 \mathrm{E}-02$ & 2.50 & 8.79 & $1.74 \mathrm{E}-03$ & $1.04 \mathrm{E}-02$ \\
\hline miR-1975 & 1.95 & 8.23 & $9.02 \mathrm{E}-03$ & $2.43 \mathrm{E}-02$ & 2.74 & 10.34 & $2.87 \mathrm{E}-03$ & $1.53 \mathrm{E}-02$ \\
\hline miR-215 & 0.20 & 0.13 & $1.12 \mathrm{E}-02$ & $2.95 \mathrm{E}-02$ & 0.42 & 0.28 & $1.62 \mathrm{E}-04$ & $1.42 \mathrm{E}-03$ \\
\hline miR-574-3p & -3.47 & 0.45 & $1.45 \mathrm{E}-02$ & $3.69 \mathrm{E}-02$ & -3.64 & 4.01 & $9.92 \mathrm{E}-03$ & 4.33E-02 \\
\hline miR-106b & 2.79 & 7.45 & $1.57 \mathrm{E}-02$ & $3.92 \mathrm{E}-02$ & 3.09 & 9.77 & $1.98 \mathrm{E}-04$ & $1.63 \mathrm{E}-03$ \\
\hline $\mathrm{miR}-450 \mathrm{a}$ & 0.12 & 0.08 & $1.63 \mathrm{E}-02$ & $4.05 \mathrm{E}-02$ & 2.42 & 1.54 & $2.00 \mathrm{E}-14$ & 4.69E-13 \\
\hline miR-1979 & 1.10 & 7.99 & $1.84 \mathrm{E}-02$ & $4.38 \mathrm{E}-02$ & 2.29 & 9.62 & $2.25 \mathrm{E}-03$ & $1.27 \mathrm{E}-02$ \\
\hline miR-2054 & 0.15 & 0.10 & $1.94 \mathrm{E}-02$ & $4.56 \mathrm{E}-02$ & 0.28 & 0.18 & $1.20 \mathrm{E}-03$ & $7.59 \mathrm{E}-03$ \\
\hline $\operatorname{miR}-181 \mathrm{c}$ & 0.15 & 0.10 & $2.07 \mathrm{E}-02$ & $4.76 \mathrm{E}-02$ & 4.10 & 3.62 & $5.15 \mathrm{E}-03$ & $2.49 \mathrm{E}-02$ \\
\hline \multicolumn{9}{|l|}{ EBV } \\
\hline miR-BART7 & 13.62 & 8.74 & $2.83 \mathrm{E}-68$ & $1.25 \mathrm{E}-65$ & 14.96 & 9.86 & $2.45 \mathrm{E}-69$ & $1.27 \mathrm{E}-66$ \\
\hline miR-BART9 & 14.49 & 9.29 & $1.10 \mathrm{E}-66$ & $2.43 \mathrm{E}-64$ & 2.15 & 8.65 & $1.65 \mathrm{E}-04$ & $1.42 \mathrm{E}-03$ \\
\hline miR-BART3 & 12.81 & 8.22 & $1.49 \mathrm{E}-63$ & $2.19 \mathrm{E}-61$ & 12.50 & 8.25 & $2.31 \mathrm{E}-61$ & $1.99 \mathrm{E}-59$ \\
\hline miR-BART22 & 11.81 & 7.58 & $9.79 \mathrm{E}-60$ & $1.08 \mathrm{E}-57$ & 15.33 & 10.10 & $1.63 \mathrm{E}-68$ & $4.21 \mathrm{E}-66$ \\
\hline miR-BART6-3p & 10.10 & 6.48 & $1.63 \mathrm{E}-54$ & $1.44 \mathrm{E}-52$ & 12.57 & 8.30 & $1.52 \mathrm{E}-63$ & $1.96 \mathrm{E}-61$ \\
\hline miR-BART17-3p & 9.54 & 6.12 & $1.74 \mathrm{E}-52$ & $1.28 \mathrm{E}-50$ & 13.13 & 8.65 & $6.87 \mathrm{E}-64$ & $1.18 \mathrm{E}-61$ \\
\hline miR-BART19-3p & 9.56 & 6.13 & $2.16 \mathrm{E}-52$ & $1.37 \mathrm{E}-50$ & 12.11 & 7.96 & $3.94 \mathrm{E}-59$ & $2.91 \mathrm{E}-57$ \\
\hline miR-BART1-3p & 9.69 & 6.21 & $1.30 \mathrm{E}-49$ & $6.36 \mathrm{E}-48$ & 12.89 & 8.49 & $3.01 \mathrm{E}-63$ & $3.11 \mathrm{E}-61$ \\
\hline miR-BART4 & 9.46 & 6.07 & $8.89 \mathrm{E}-49$ & $3.93 \mathrm{E}-47$ & 11.30 & 7.46 & $1.28 \mathrm{E}-57$ & $8.24 \mathrm{E}-56$ \\
\hline miR-BART18-5p & 9.03 & 5.79 & $6.03 \mathrm{E}-46$ & $2.42 \mathrm{E}-44$ & 11.24 & 7.40 & $3.53 \mathrm{E}-57$ & $2.03 \mathrm{E}-55$ \\
\hline miR-BART21-3p & 8.52 & 5.46 & $2.30 \mathrm{E}-45$ & $8.46 \mathrm{E}-44$ & 11.05 & 7.27 & $1.14 \mathrm{E}-55$ & $5.89 \mathrm{E}-54$ \\
\hline miR-BART11-5p & 6.35 & 4.08 & $3.77 \mathrm{E}-33$ & $1.19 \mathrm{E}-31$ & 10.32 & 6.78 & $1.29 \mathrm{E}-52$ & $6.07 \mathrm{E}-51$ \\
\hline miR-BART17-5p & 6.25 & 4.01 & $3.04 \mathrm{E}-32$ & $8.52 \mathrm{E}-31$ & 10.38 & 6.87 & $3.22 \mathrm{E}-47$ & $1.39 \mathrm{E}-45$ \\
\hline miR-BART15 & 4.20 & 2.69 & $2.57 \mathrm{E}-22$ & $5.17 \mathrm{E}-21$ & 7.87 & 5.18 & $4.08 \mathrm{E}-42$ & $1.62 \mathrm{E}-40$ \\
\hline miR-BART14 & 2.14 & 1.37 & $2.86 \mathrm{E}-12$ & $3.42 \mathrm{E}-11$ & 3.53 & 2.32 & $2.18 \mathrm{E}-18$ & $6.26 \mathrm{E}-17$ \\
\hline miR-BART18-3p & 1.70 & 1.09 & $1.50 \mathrm{E}-09$ & $1.58 \mathrm{E}-08$ & 3.62 & 2.37 & $4.73 \mathrm{E}-18$ & $1.29 \mathrm{E}-16$ \\
\hline miR-BART6-5p & 9.01 & 6.58 & $5.79 \mathrm{E}-08$ & 4.92E-07 & 4.98 & 9.41 & $9.17 \mathrm{E}-07$ & $1.06 \mathrm{E}-05$ \\
\hline miR-BART19-5p & 1.98 & 1.27 & 7.03E-08 & $5.87 \mathrm{E}-07$ & 4.07 & 2.61 & $3.33 \mathrm{E}-15$ & $8.20 \mathrm{E}-14$ \\
\hline miR-BART5 & 3.45 & 9.14 & $1.88 \mathrm{E}-07$ & $1.51 \mathrm{E}-06$ & 5.26 & 3.46 & $4.78 \mathrm{E}-27$ & $1.54 \mathrm{E}-25$ \\
\hline miR-BART1-5p & 1.19 & 0.76 & $1.13 \mathrm{E}-06$ & $8.34 \mathrm{E}-06$ & 4.83 & 3.20 & $3.42 \mathrm{E}-23$ & $1.04 \mathrm{E}-21$ \\
\hline miR-BART8 & 10.73 & 8.05 & $2.22 \mathrm{E}-06$ & $1.49 \mathrm{E}-05$ & 14.46 & 10.09 & $2.36 \mathrm{E}-11$ & $4.36 \mathrm{E}-10$ \\
\hline miR-BART2-3p & 0.77 & 0.49 & $5.83 \mathrm{E}-06$ & $3.68 \mathrm{E}-05$ & 1.82 & 1.22 & $2.48 \mathrm{E}-10$ & $3.88 \mathrm{E}-09$ \\
\hline miR-BART10 & 3.42 & 9.19 & $8.72 \mathrm{E}-05$ & $4.33 \mathrm{E}-04$ & 6.87 & 10.40 & $1.12 \mathrm{E}-03$ & 7.34E-03 \\
\hline miR-BART13 & 0.74 & 0.47 & $1.49 \mathrm{E}-04$ & $7.00 \mathrm{E}-04$ & 2.09 & 1.35 & $1.94 \mathrm{E}-11$ & $3.85 \mathrm{E}-10$ \\
\hline miR-BART12 & 0.36 & 0.23 & $3.10 \mathrm{E}-03$ & $9.94 \mathrm{E}-03$ & 5.79 & 3.84 & $1.26 \mathrm{E}-29$ & 4.34E-28 \\
\hline miR-BART20-3p & 0.31 & 0.20 & $3.29 \mathrm{E}-03$ & $1.04 \mathrm{E}-02$ & 0.72 & 0.46 & $1.04 \mathrm{E}-04$ & $9.62 \mathrm{E}-04$ \\
\hline miR-BART11-3p & 0.36 & 0.23 & $7.83 \mathrm{E}-03$ & $2.17 \mathrm{E}-02$ & 0.53 & 0.34 & $9.85 \mathrm{E}-04$ & $6.65 \mathrm{E}-03$ \\
\hline miR-BART21-5p & 0.23 & 0.14 & $1.55 \mathrm{E}-02$ & $3.90 \mathrm{E}-02$ & 2.20 & 1.42 & $2.09 \mathrm{E}-11$ & $4.01 \mathrm{E}-10$ \\
\hline \multicolumn{9}{|l|}{ Other Viruses } \\
\hline hsv1-miR-H8 & 4.11 & 2.63 & $1.19 \mathrm{E}-23$ & $2.51 \mathrm{E}-22$ & 1.54 & 6.75 & $8.37 \mathrm{E}-04$ & $5.93 \mathrm{E}-03$ \\
\hline hcmv-miR-UL22A & 2.79 & 1.79 & $2.30 \mathrm{E}-14$ & $3.18 \mathrm{E}-13$ & 0.26 & 0.21 & $3.97 \mathrm{E}-03$ & $2.01 \mathrm{E}-02$ \\
\hline
\end{tabular}




\subsubsection{Generation and validation of miRNA signature associated with risk of distant metastasis}

Using the Lasso method to fit a penalized CoxPH model to miRNA expression data from the Training cohort yielded 33 variables (miRNAs) with non-zero coefficients (Table 3-3). The 4 miRNAs most strongly associated (greatest absolute coefficient values) with risk of DM were combined with their coefficients within the penalized model to yield the following equation:

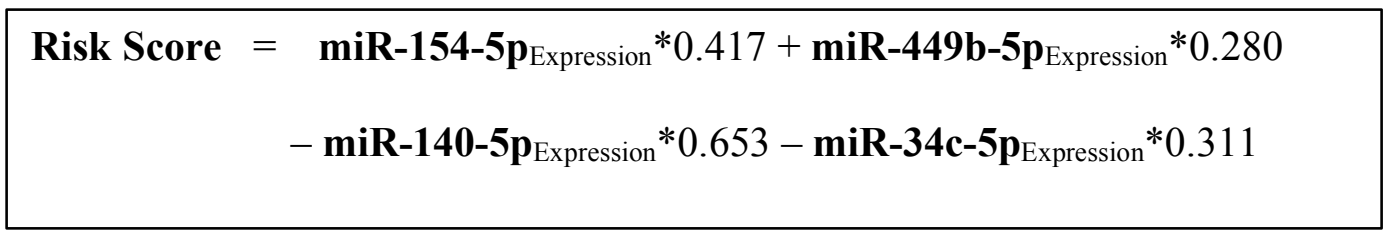

The RS was calculated for each patient in the Training cohort, allowing the patients to be dichotomized into either a "low risk" ( $<$ median), or a "high risk" $(\geq$ median) group. A highly significant relationship was observed between the RS and the likelihood of DM whether the RS was treated as a continuous (Wald test; $\mathrm{HR}=2.76 ; \mathrm{p}=2.8 \times 10^{-5}$ ), or a binary (log-rank test: $\left.\mathrm{HR}=8.25 ; \mathrm{p}=8.0 \times 10^{-4}\right)$ variable $($ Fig $3-1 \mathrm{~A})$.

When the same miRNA signature equation was applied to the independent Validation cohort of 121 NPC patients diagnosed approximately a decade earlier, a similar relationship was observed (Fig 3-1B). When treated as a continuous predictor, the HR was 1.7 with a Wald test $\mathrm{p}$-value $=0.05$. When dichotomized by the median from the training cohort, the HR was 3.2 , with a log-rank test p-value of 0.014 . These results indicate that there might be a greater benefit to utilizing this RS as a binary variable, with an established cut-point, as opposed to a continuous spectrum of risk. 
When this 4-miRNA signature was examined further in miRNA-Seq expression data generated by TCGA, there was a significant difference observed in disease-specific survival for patients with a "high risk" score compared to those with a "low risk" score $(\mathrm{HR}=1.8 ; \mathrm{p}=0.02$; Fig 3-2), despite the fact that none of these samples were NPCs with the majority derived from the oral cavity or larynx. 
Table 3-3. MiRNAs with non-zero coefficients in the penalized regression model fitted to miRNA expression data from the training set.

\begin{tabular}{|l|l|}
\hline miRNA & Penalized Coefficient \\
\hline hsa-miR-140-5p & -0.65265805 \\
hsa-miR-154 & 0.416841936 \\
hsa-miR-34c-5p & -0.311288747 \\
hsa-miR-449b & 0.27970981 \\
hsa-miR-136 & 0.278252522 \\
hsa-miR-1246 & 0.252954521 \\
hsa-miR-31 & 0.246736327 \\
hsa-miR-633 & -0.232982777 \\
hsa-miR-375 & 0.227348203 \\
hsa-miR-646 & -0.187297587 \\
hsa-miR-188-5p & -0.184079758 \\
hsa-miR-574-3p & 0.167259093 \\
hsa-miR-542-3p & -0.165407907 \\
hsa-miR-1 & 0.153553988 \\
hsa-miR-561 & -0.152880207 \\
hsa-miR-580 & -0.147656138 \\
hsa-miR-155 & -0.145379233 \\
hsa-miR-34c-3p & 0.114592858 \\
hsa-miR-196a & -0.099335183 \\
hsa-miR-296-5p & 0.094110203 \\
hsa-miR-107 & 0.093446126 \\
hsa-miR-663b & 0.074681673 \\
hsa-miR-600 & 0.063613683 \\
hsa-miR-142-5p & -0.060944673 \\
hsa-miR-132 & -0.056635448 \\
ebv-miR-BART14 & 0.048490936 \\
hsa-miR-362-5p & 0.043422478 \\
hsa-miR-377 & 0.04287268 \\
hsa-miR-934 & -0.036495034 \\
hsa-miR-144 & 0.034527264 \\
hsa-miR-188-3p & -0.033462301 \\
hsa-miR-93 & 0.007480249 \\
hsa-miR-579 & -0.003676771 \\
\hline
\end{tabular}




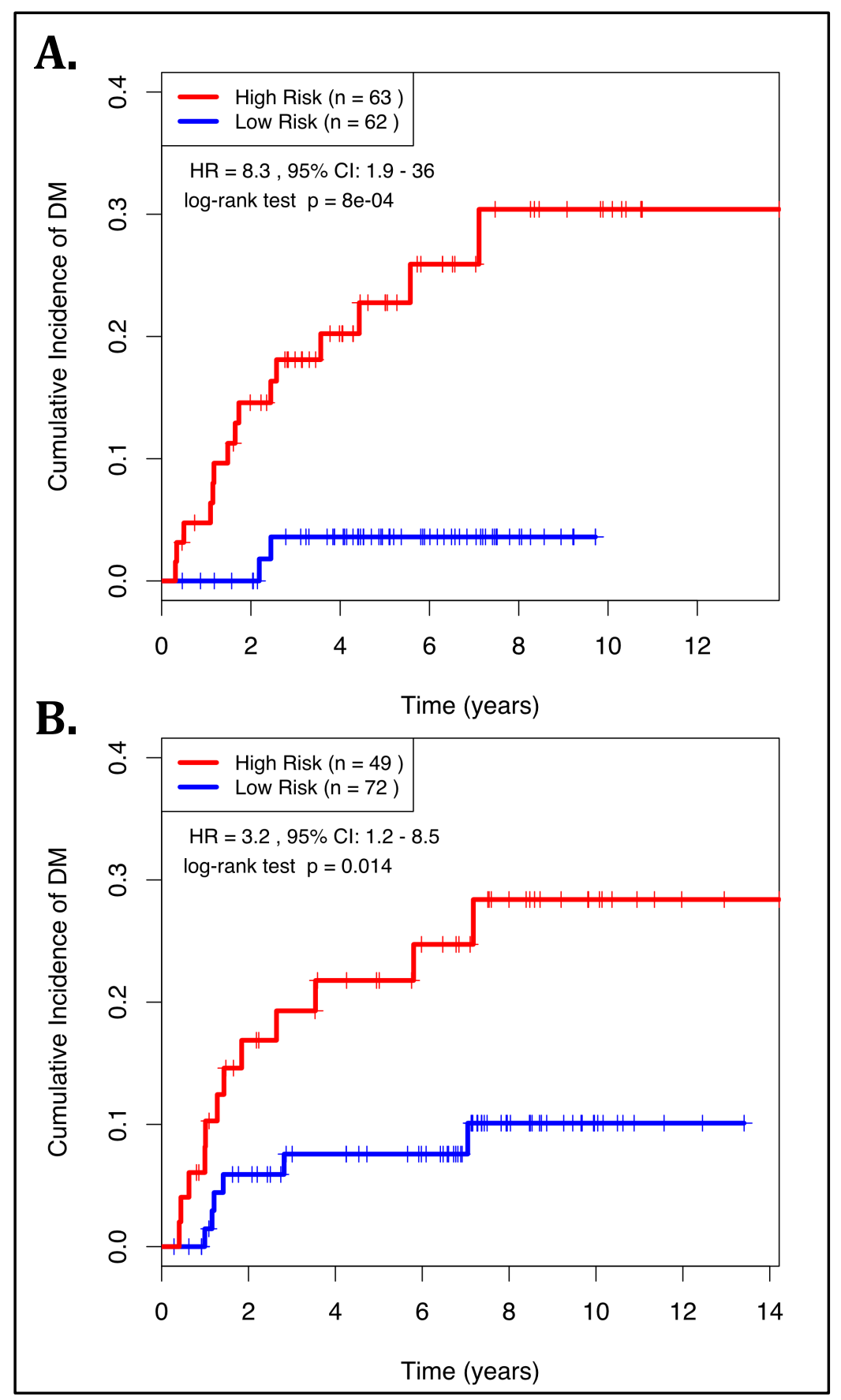

Figure 3-1. Kaplan-Meier curves showing NPC patients dichotomized based on risk score A) Training cohort; and B) Validation cohort. "High Risk" is defined as a RS $\geq$ the median in the training cohort, and "Low Risk" is defined as a RS $<$ the median in the training cohort. RS; Risk Score, HR; Hazard Ratio, CI; Confidence Interval. 


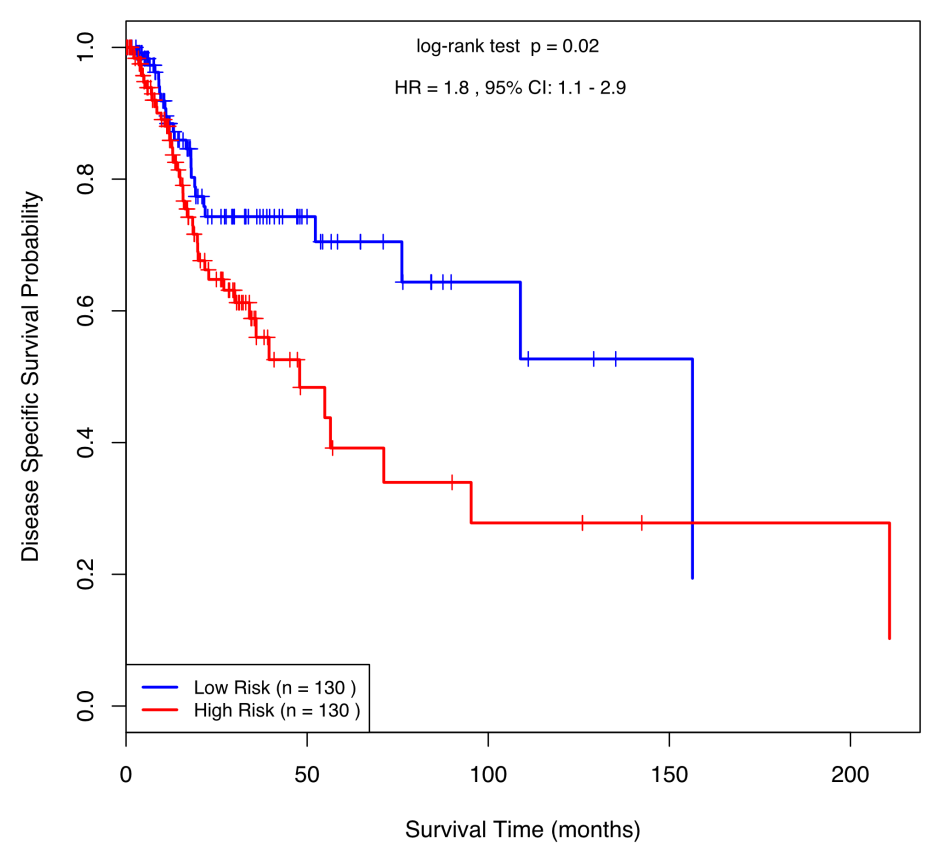

Figure 3-2. Kaplan-Meier curves demonstrating the association Disease Specific Survival the 4-miRNA signature applied to HNSCC miRNA-Seq Data generated by TCGA.

"High Risk" is defined as a RS $\geq$ the median, and "Low Risk" is defined as a RS $<$ the median. RS; Risk Score, HR; Hazard Ratio, CI; Confidence Interval. 


\subsubsection{Multivariate Analysis}

Table 3-4 demonstrates that after controlling for clinical factors such as stage, age, gender, and treatment, the miRNA signature RS remained significantly associated with risk of DM $\left(\mathrm{HR}=4.0 ; \mathrm{p}=7.1 \times 10^{-4}\right)$. Nodal stage was the only other factor significantly associated with DM on both univariate and multivariate analyses (Table 3-2). For this reason, the 4-miRNA signature RS was then combined with N-stage to define five risk groups, based on the visual inspection of the DMFS Kaplan-Meier curves stratified by N-stage (Fig 3-3). Figure 3-4A demonstrates that when patients were stratified into these five distinct groups, the patients in the N1/N2 Group and the N3 Group were further dichotomized by the miRNA RS, thus depicting the ability of this 4-miRNA signature to provide improved risk prediction for DM in these clinically intermediate and high-risk groups. This improved prognostic ability was further illustrated when ROC AUCs were calculated over time and compared amongst clinical factors, the original 4-miRNA RS, and the 5-group N-Stage/miRNA risk-stratification (Fig 3-4B). The area under the ROC curves was consistently greatest in this 5-group stratification (save for year-1, where the 4-miRNA signature alone marginally outperformed the model including Nstage). C-statistics for each CoxPH model were also calculated (Table 3-5), further demonstrating the significantly greater prognostic value for this combined parameter (c-statistic $=0.78)$, compared to all other models. 
Table 3-4. Univariate and multivariate CoxPH analysis of clinical factors and miRNAsignature Risk-Score in the combined dataset from both the Training and Validation cohorts $(n=242)$.

\begin{tabular}{|l|cc|cc|}
\hline \multicolumn{1}{|c}{ Univariate } & \multicolumn{2}{c|}{ Multivariate } \\
\hline & HR (95\% Cl) & p-value & HR (95\% Cl) & p-value \\
\hline MiRNA RS (High Risk vs. Low Risk) & $4.5(2.04-9.90)$ & $1.9 \times 10^{-4}$ & $4.0(1.8-8.9)$ & $7.3 \times 10^{-4}$ \\
T stage (T1\&2 vs. T3\&4) & $1.7(0.86-3.3)$ & 0.13 & $1.5(0.70-3.2)$ & 0.30 \\
N stage (N0\&1 vs. N2\&3) & $2.5(1.2-5.0)$ & $8.0 \times 10^{-3}$ & $2.7(1.2-5.7)$ & 0.01 \\
Age & $1.0(0.98-1.0)$ & 0.86 & $1.0(0.98-1.0)$ & 0.93 \\
Gender (Female vs. Male) & $1.31(0.60-2.9)$ & 0.50 & $1.3(0.57-2.9)$ & 0.53 \\
Chemotherapy (- vs. +) & $0.97(0.5-1.9)$ & 0.94 & $0.46(0.21-1.0)$ & 0.05 \\
\hline
\end{tabular}




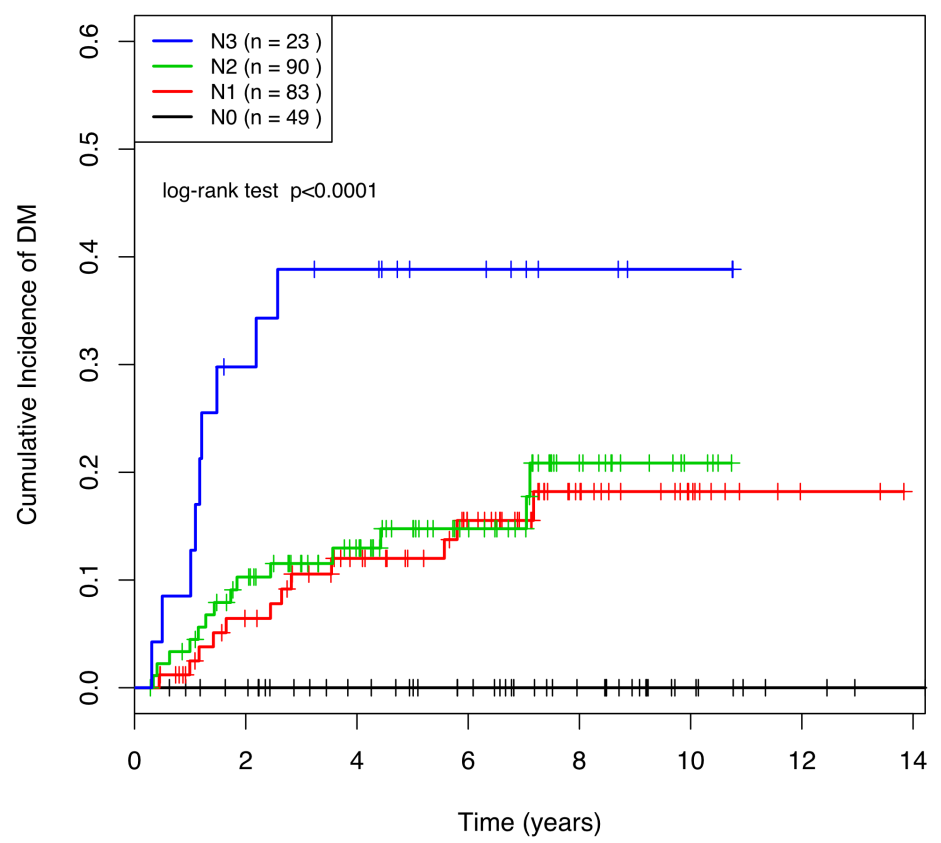

Figure 3-3. Kaplan-Meier curves demonstrating cumulative incidence of distant metastasis based on clinical Nodal-stage in the combined dataset $(n=245)$. 


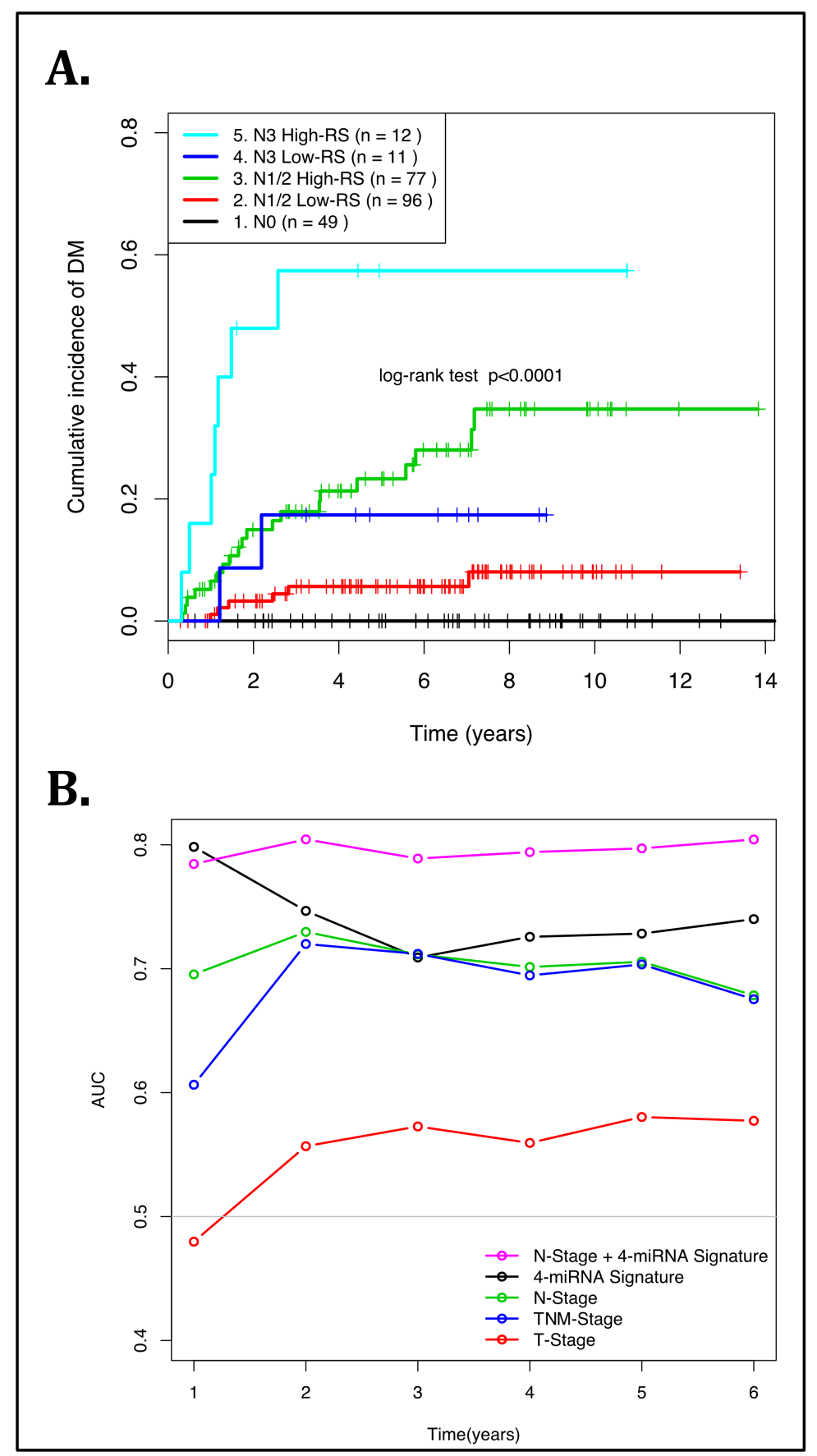

Figure 3-4. Comparison of 4-miRNA signature with clinical variables.

A) DM Kaplan-Meier curve showing patients assigned to risk groups based on the combined Nstage and 4-miRNA signature Risk Score. B) ROC AUCs over time demonstrating the ability of various clinical factors and the 4-miRNA signature RS to predict DM in NPC patients. DM; distant metastasis, ROC; receiver operating characteristic, AUC; Area Under the Curve, RS; Risk Score. 
Table 3-5. C-statistics for comparison of various CoxPH models' ability to predict DMFS.

\begin{tabular}{|c|c|}
\hline Covariates included in the model (levels for each variable) & C-Statistic \pm SE \\
\hline $\begin{array}{l}\text { 4-miRNA Signature (High Risk/Low Risk), } \\
\text { N-stage (N0\&1/N2\&3) }\end{array}$ & $0.78 \pm 0.048$ \\
\hline $\begin{array}{l}\text { 5-miRNA Liu et al. Signature (High Risk/Low Risk), } \\
\text { N-stage (N0\&1/N2\&3) }\end{array}$ & $0.74 \pm 0.048$ \\
\hline 4-miRNA Signature (High Risk/Low Risk) & $0.71 \pm 0.050$ \\
\hline TNM-stage (I/II/III/IV) & $0.67 \pm 0.049$ \\
\hline $\mathrm{N}$-stage (N0\&1/N2\&3) & $0.62 \pm 0.043$ \\
\hline 5-miRNA Liu et al. Signature (High Risk/Low Risk) & $0.60 \pm 0.044$ \\
\hline T-Stage (T1\&2/T3\&4) & $0.55 \pm 0.043$ \\
\hline
\end{tabular}

Figure 3-5 demonstrates the ability of this 4-miRNA signature to predict risk of DM in advanced stage (III \& IV) patients who were treated with RT alone (HR=7.13; p=0.003; Fig 35A), as well as those who were treated with $\mathrm{CRT}(\mathrm{HR}=3.35 ; \mathrm{p}=0.045$; Fig 3-5B). These data suggest that even in NPC patients with locally-advanced disease, this 4-miRNA signature could potentially identify a low-risk group of patients, whose likelihood of developing DM at 5-years was so low $(<10 \%)$, that adding CT to their RT might have no benefit to their survival (Fig 35A). Conversely, there remained a high-risk group, whose outcome could be potentially improved with the administration of combined CRT, since RT alone was associated with a 5year risk of $45 \%$ in developing DM (Fig 3-5A), vs. 20\% when treated with CRT (Fig 3-5B). 


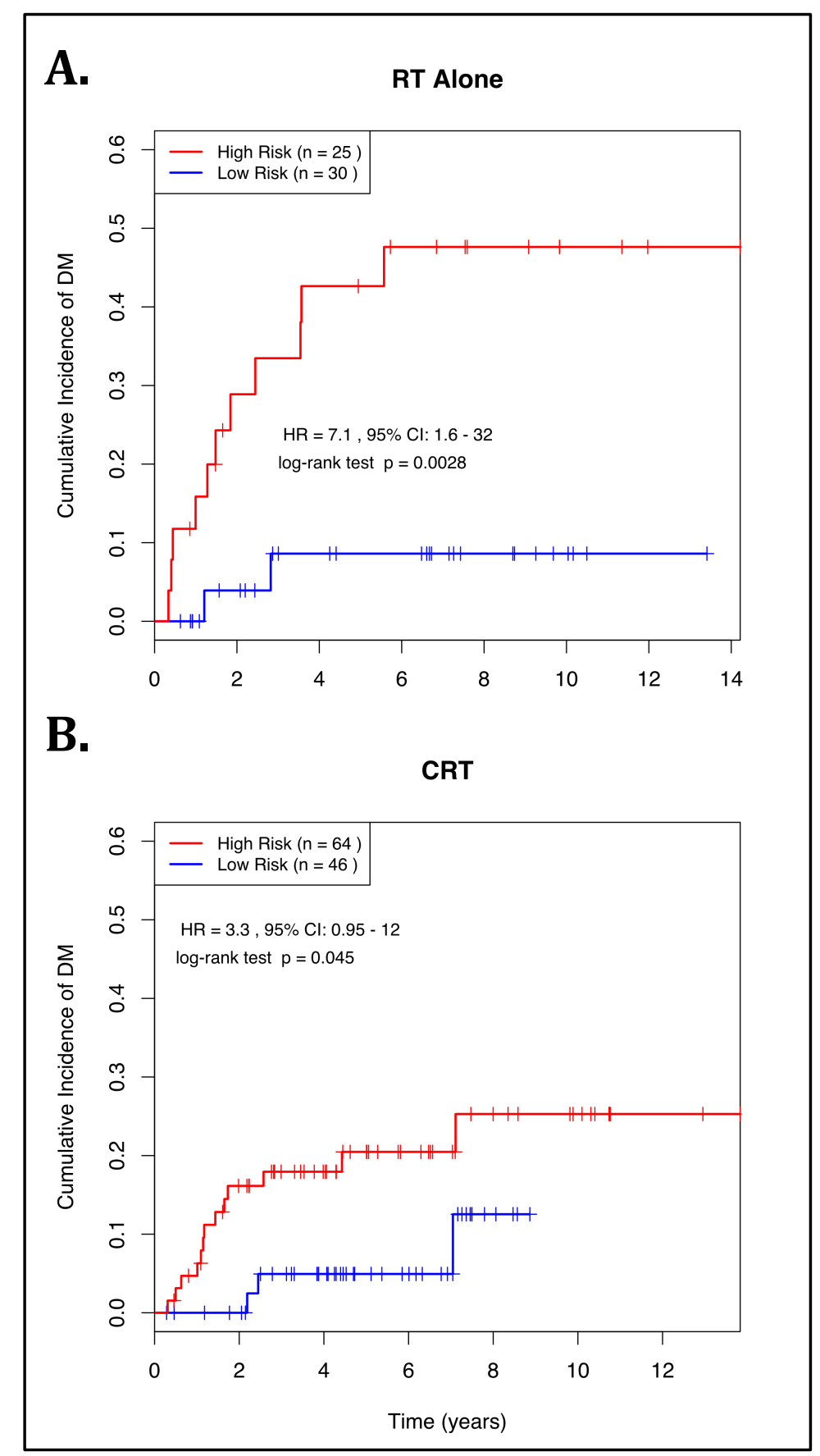

Figure 3-5. Kaplan-Meier curves showing DM in NPC patients dichotomized based on miRNA risk score in advanced stage patients (Stage III/IV).

Patients treated with A) RT alone; and B) combined CRT. "High Risk" is defined as a RS $\geq$ the median in the training set, and "Low Risk" is defined as a RS $<$ the median in the training set. RT; radiotherapy, CRT; chemoradiotherapy, RS; Risk Score, HR; Hazard Ratio, CI; Confidence Interval. 


\subsubsection{Validated miRNA Target Pathway Enrichment Analysis}

When the miRWalk database was queried, 292 miR-target relationships were identified for the 4 miRNAs in the prognostic signature. When pathway enrichment analysis was performed, several biological pathways were observed to be significantly enriched (corrected pvalue $<0.05$ ) within the union of validated targets for all 4 miRNAs (Table 3-6). The "Cell Cycle" KEGG pathway, and the "Cell Cycle: G1/S Check Point" Biocarta pathways were the first and fourth most significantly enriched pathways, respectively. These were also the only two pathways identified to be significantly enriched in the targets for 3 of the 4 miRNAs (miR449b-5p, miR-34c-5p, miR-140-5p) when individual pathway analysis was also performed, suggesting that the underlying biological processes of cell cycling or cellular proliferation may be mediating the clinical observations. 
Table 3-6. Pathways observed to be significantly enriched within validated targets of the four miRNAs in the prognostic signature using DAVID (v6.7).

\begin{tabular}{|c|c|c|c|c|c|}
\hline Database & Term & Count & $\begin{array}{c}\text { Fold } \\
\text { Enrichment }\end{array}$ & $\begin{array}{c}\text { Raw } \\
\text { p-value }\end{array}$ & $\begin{array}{l}\text { Corrected } \\
\text { p-value }\end{array}$ \\
\hline KEGG_PATHWAY & Cell cycle & 22 & 4.23 & 3.97E-09 & 4.44E-07 \\
\hline KEGG_PATHWAY & Melanoma & 18 & 4.38 & 1.02E-07 & $5.72 \mathrm{E}-06$ \\
\hline KEGG_PATHWAY & Pathways in cancer & 38 & 2.3 & 1.69E-07 & $6.30 \mathrm{E}-06$ \\
\hline BIOCARTA & Cell Cycle: G1/S Check Point & 15 & 5.09 & 3.76E-08 & $6.95 \mathrm{E}-06$ \\
\hline KEGG_PATHWAY & Glioma & 16 & 4.39 & 6.83E-07 & $1.91 \mathrm{E}-05$ \\
\hline KEGG_PATHWAY & Pancreatic cancer & 17 & 3.91 & $1.52 E-06$ & $3.40 E-05$ \\
\hline KEGG_PATHWAY & Chronic myeloid leukemia & 17 & 3.71 & 3.29E-06 & $6.13 E-05$ \\
\hline KEGG_PATHWAY & Bladder cancer & 13 & 4.79 & 4.26E-06 & $6.82 \mathrm{E}-05$ \\
\hline KEGG_PATHWAY & Colorectal cancer & 17 & 3.53 & 6.73E-06 & $9.43 E-05$ \\
\hline KEGG_PATHWAY & Prostate cancer & 18 & 3.31 & 8.27E-06 & $1.03 E-04$ \\
\hline BIOCARTA & p53 Signaling Pathway & 11 & 5.27 & 3.92E-06 & $3.63 E-04$ \\
\hline KEGG_PATHWAY & p53 signaling pathway & 13 & 3.72 & 7.61E-05 & $8.52 E-04$ \\
\hline BIOCARTA & ATM Signaling Pathway & 10 & 5.09 & 2.11E-05 & $1.30 \mathrm{E}-03$ \\
\hline PANTHER_PATHWAY & p53 pathway & 16 & 3.07 & $5.75 \mathrm{E}-05$ & $1.70 \mathrm{E}-03$ \\
\hline PANTHER_PATHWAY & p53 pathway feedback loops 2 & 13 & 3.92 & 3.02E-05 & $1.78 \mathrm{E}-03$ \\
\hline KEGG_PATHWAY & Non-small cell lung cancer & 12 & 3.6 & 2.34E-04 & $2.38 \mathrm{E}-03$ \\
\hline KEGG_PATHWAY & Small cell lung cancer & 14 & 3.11 & 2.62E-04 & $2.44 \mathrm{E}-03$ \\
\hline BIOCARTA & Cyclins and Cell Cycle Regulation & 10 & 4.52 & 7.27E-05 & $3.36 \mathrm{E}-03$ \\
\hline KEGG_PATHWAY & Focal adhesion & 19 & 2.35 & 5.42E-04 & 4.66E-03 \\
\hline BIOCARTA & $\begin{array}{l}\text { Role of BRCA1, BRCA2 and ATR in } \\
\text { Cancer Susceptibility }\end{array}$ & 7 & 5.18 & 7.49E-04 & $2.73 E-02$ \\
\hline KEGG_PATHWAY & Notch signaling pathway & 7 & 4.3 & 3.74E-03 & 2.95E-02 \\
\hline KEGG_PATHWAY & Thyroid cancer & 7 & 4.3 & 3.74E-03 & $2.95 \mathrm{E}-02$ \\
\hline BIOCARTA & $\begin{array}{l}\text { Telomeres, Telomerase, Cellular } \\
\text { Aging, and Immortality }\end{array}$ & 7 & 4.75 & 1.35E-03 & 4.09E-02 \\
\hline
\end{tabular}




\subsection{Discussion}

Given the continued challenge of DM in NPC patients, the validated prognostic 4miRNA signature presented herein could prove to be a valuable tool in guiding treatment decisions for patients. To date, there are no clinically utilized prognostic biomarkers in NPC; plasma levels of EBV DNA titres have certainly been associated with clinical outcome, particularly in predicting risk of relapse when the titre remained elevated, or increased posttreatment ${ }^{128,300}$. However, this has not contributed to treatment decisions, which to date, have relied on TNM staging, with administration of combined CRT for patients with locallyadvanced NPC. However, such CRT regimens are also associated with significant acute and long-term morbidity, and even mortality, diluting any potential gains with these aggressive treatments ${ }^{318-320}$. Hence, the utility of this 4-miRNA signature, which has been validated in an independent cohort (Fig 3-1) and was demonstrated to add to the prognostic value of the TNM staging category (Fig 3-3), is extremely promising. Furthermore, the apparent ability of this 4miRNA signature to identify a low-risk group of patients with locally-advanced NPC, who could be cured with RT alone (Fig 3-5), suggests a potential predictive value of this signature, which to date, has never been previously documented. However, given the inherent risk of selection bias that comes with a retrospective study such as this, in order to confirm the ability of this signature to predict treatment success for patients treated with RT alone, further verification in an additional independent cohort of randomized controlled trial (RCT) participants randomly designated to RT alone vs. CRT arms would be needed. This would potentially make it possible to de-escalate treatment for patients with truly a favourable prognosis, thus sparing them the increased toxicity of combined CRT. In contrast, patients at high-risk will have a $60 \%$ risk of DM at 5-years, despite CRT; for this group of patients, the 
need to identify and evaluate novel therapeutics cannot be over-emphasized. Indeed, identifying such targetable pathways may be achieved by probing the underlying biology of these miRNA signatures.

There exists another validated prognostic 5-miRNA signature set for NPC, generated by a group from Guangdong, which was associated with overall, disease-free, and distant metastasis-free survival ${ }^{183}$. We have evaluated their 5-miRNA signature, compared to either our 4-miRNA signature alone, or each combined with N-stage, and our signatures appeared to demonstrate a more robust performance, based on direct comparisons and a ROC curve analysis (Fig 3-6, and Table 3-6). Specifically, comparing the HR of our 4-miRNA signature to the HR of the 5-miRNA signature for DM (Fig 3-7A vs. B), our current 4-miRNA signature appeared to perform better, with a higher HR and greater statistical significance. Similarly, the greatest AUC in the ROC analysis was observed with our 4-miRNA signature combined with N-stage (Fig 3-7C). Of note, there is a complete lack of overlap between their 5-miRNAs and our 4miRNAs. This is not surprising, given the differences in platforms (in-house vs. Nanostring nCounter), different population cohorts, and redundancy in biological processes; this phenomenon has been similarly observed with other miRNA signatures ${ }^{321,322}$, as well as mRNA signatures $^{323}$. Future analysis on additional independent datasets would be required to definitively determine the optimal signature; as well as the potential importance of geographical and population differences. 


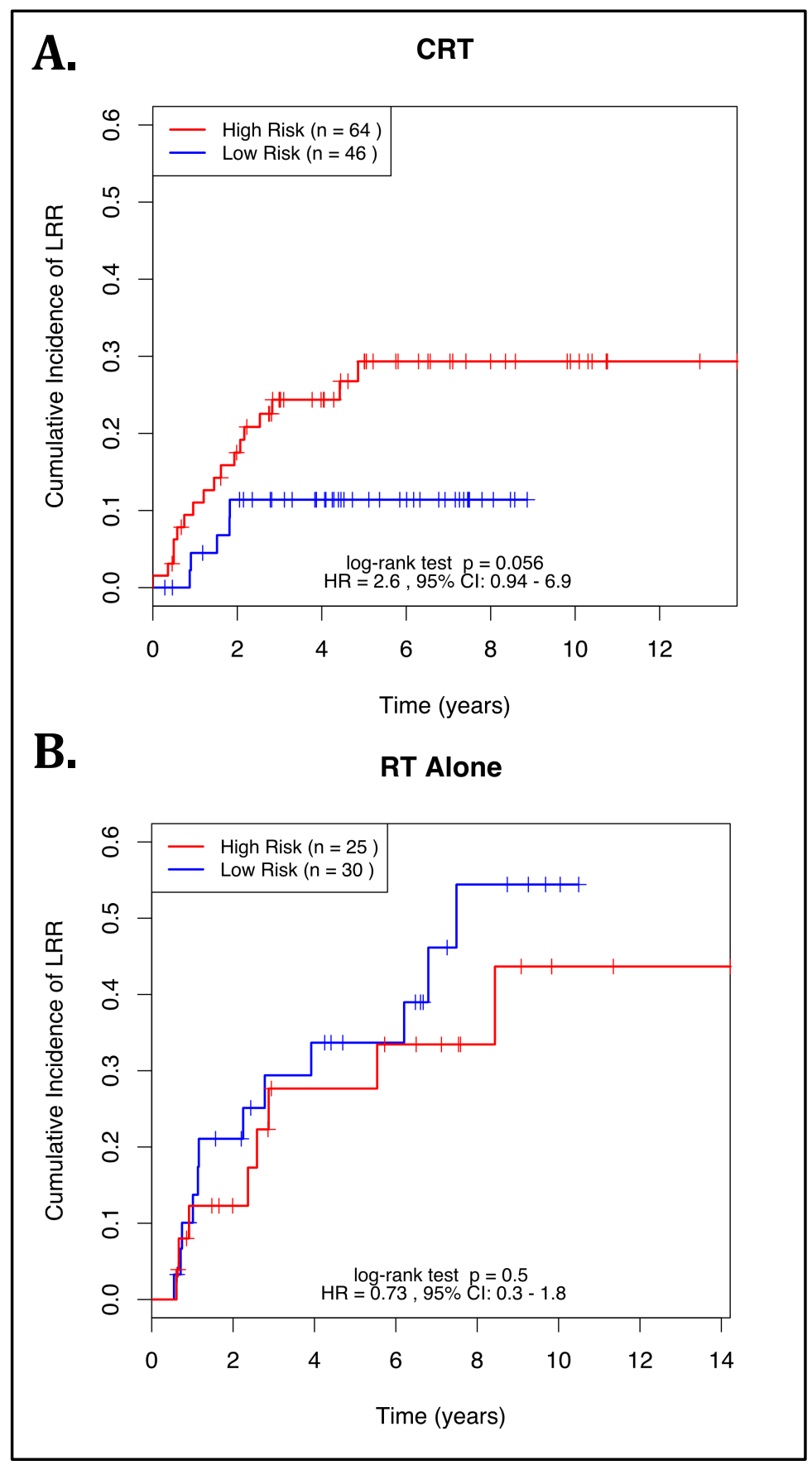

Figure 3-6. Kaplan-Meier curves showing local-regional relapse (LRR) in NPC patients dichotomized based on miRNA RS in advanced stage patients (Stage III/IV).

Patients treated with A) RT alone and B) combined CRT. "High Risk" is defined as RS $\geq$ the median in the training set, and "Low Risk" is defined as a RS $<$ the median in the training set. RT; radiotherapy, CRT; chemoradiotherapy, HR; Hazard Ratio, CI; Confidence Interval. 

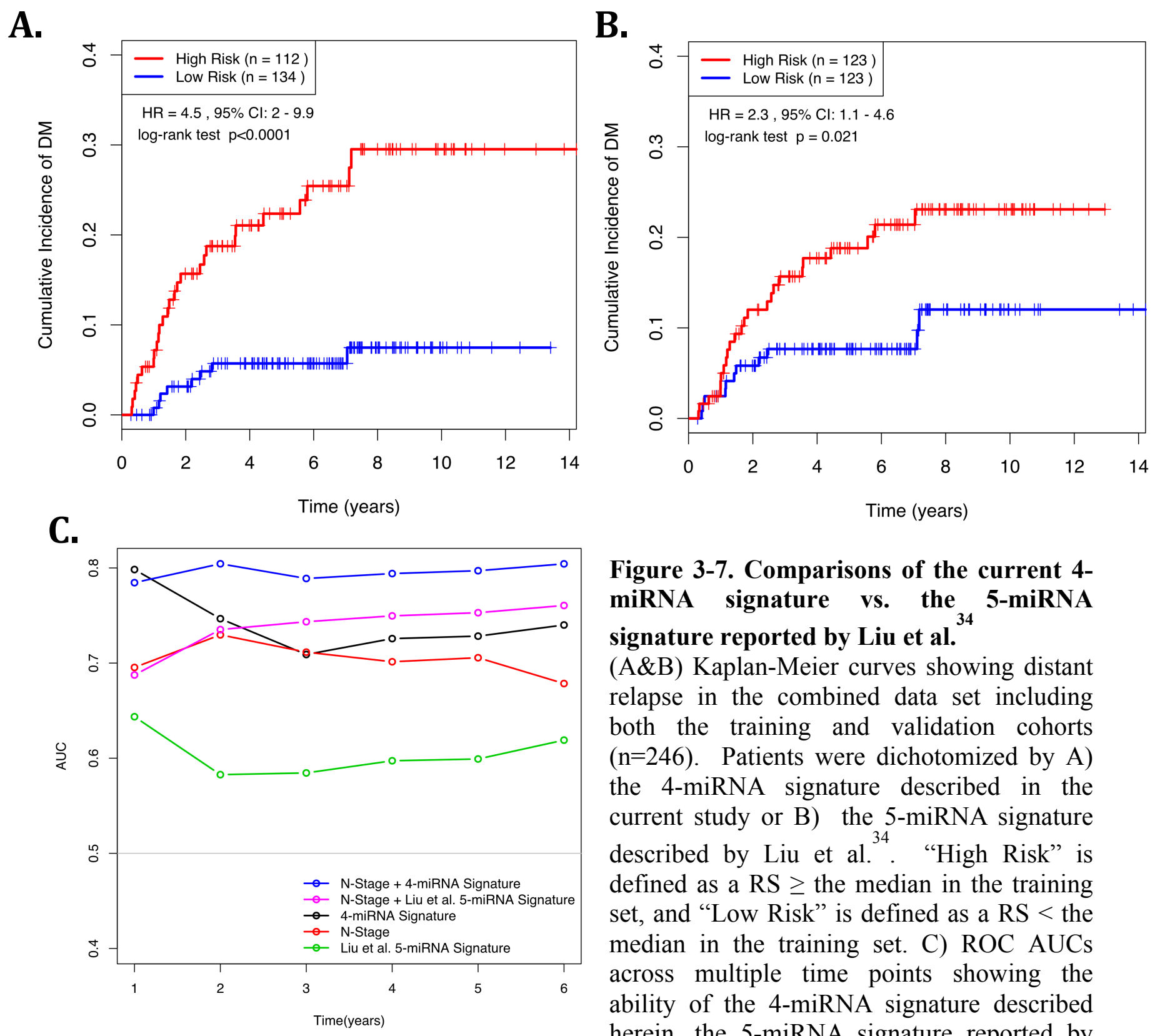

Figure 3-7. Comparisons of the current 4miRNA signature vs. the 5-miRNA signature reported by Liu et al.

(A\&B) Kaplan-Meier curves showing distant relapse in the combined data set including both the training and validation cohorts $(n=246)$. Patients were dichotomized by A) the 4-miRNA signature described in the current study or B) the 5-miRNA signature described by Liu et al. . "High Risk" is defined as a $R S \geq$ the median in the training set, and "Low Risk" is defined as a RS $<$ the median in the training set. C) ROC AUCs across multiple time points showing the ability of the 4-miRNA signature described herein, the 5-miRNA signature reported by Liu et al. ${ }^{31}$ and N-stage alone or combined with either signature to predict DM in NPC patients. These data demonstrate that the model including N-stage and the 4-miRNA signature is the most robust prognostic signature. HR; Hazard Ratio, CI; Confidence Interval, N; Nodal, ROC; receiver operating characteristic. AUC; Area under the curve, RS; Risk Score. 
Preliminary pathway enrichment analysis suggests a role in cell cycle regulation for the 4 miRNAs in this signature set. MiR-34c-5p, miR-140-5p, miR-154-5p and miR-449b-5p have all been previously reported to target important members of the cell cycle control/G1-S checkpoint pathway ${ }^{324-327}$. Given the relationship to DM reported in this study, one could potentially hypothesize that since both chemotherapy and RT primarily target actively dividing cells $^{328,329}$; the "high-risk" tumours might be rendered more resistant by exhibiting a quiescent cellular phenotype. However, this is merely a postulate, which would require extensive preclinical investigations before any conclusions could be drawn regarding the underlying biological basis of these miRNAs in promoting metastasis in NPC.

Despite the numerous publications purporting the identification of prognostic miRNA signatures (reviewed by Nair et $\mathrm{al}^{177}$ ); none to date has been utilized in the clinic. We are hopeful that this 4-miRNA signature set will be verified and implemented, based on several strengths of this current NPC study: a) reasonable sample size (both Training and Validation cohorts were in excess of 100 patients); b) uniformity of treatment at a single institution; c) identical DMFS in both cohorts despite the difference in time period and increased use of chemotherapy in the Training cohort; d) a single experimentalist to ensure technical consistency; e) the same type of tissues (both cohorts were FFPE blocks); and finally, f) consistency of the miRNA profiling platform. Indeed, if this 4-miRNA signature can be verified in yet a third independent RCT cohort, this signature could be theoretically readily translatable, given that FFPE processing is broadly utilized around the world.

In conclusion, we have successfully validated a 4-miRNA signature that can prognosticate for DM in patients with NPC. This signature adds to the prognostic value of the current "gold standard" of the TNM staging categories, and there is a suggestion of its potential 
predictive role in selecting NPC patients for de-escalating treatment to RT alone, despite locally-advanced disease at presentation. Further interrogation of the underlying biological pathways such as cell cycle and proliferation could render the selection of patients who might be sensitive to HDAC or EGFR inhibitors. Such novel molecular targeted agents have been demonstrated to have promising efficacy in the clinic $^{10,330,331}$, as well as in pre-clinical models ${ }^{332}$; the utilization of a prognostic miRNA signature would enrich for potentially sensitive patients, thereby improving clinical outcome for future patients with NPC. 


\section{Chapter 4}

\section{Genomic characterization of NPC}

Jeff P. Bruce, Angela B.Y. Hui, Bayardo Perez-Ordonez, Ilan Weinreb, Brian O’Sullivan, John Waldron, Shao Hui Huang, Ralph Gilbert, Fei-Fei Liu 


\section{Genomic characterization of NPC}

\subsection{Introduction}

In addition to the pivotal role of EBV infection in NPC tumourigenesis, a number of genetic alterations have also been established as contributors to NPC development and progression. Early genetic analysis of NPC samples focused on gross chromosomal aberrations interrogated through low-resolution genome-wide methods such as $\mathrm{PCR}^{22}$, comparative genomic hybridization $(\mathrm{CGH})^{26}$, and more recently, the higher resolution array CGH $(\mathrm{aCGH})^{43}$. These studies have identified several recurrently amplified and deleted regions, which have provided valuable insight into the genetic basis of NPC (reviewed in chapter 1). However, the quality and quantity requirements of the genomic DNA required for such techniques has limited the sample size and clinical follow-up data available for such studies ${ }^{333}$.

Recent advancements in molecular inversion probe (MIP) array technologies have made interrogating a large number of single nucleotide polymorphisms (SNPs) relatively simple and inexpensive. In addition to sequence information, intensity values for SNPs tiled across the genome can also be used to generate genome-wide copy-number data ${ }^{334}$. Moreover, specific methodologies have been developed to enable the generation of high-quality copy-number data from relatively small amounts of genomic DNA ( $<20 \mathrm{ng}$ in some cases) extracted from

formalin-fixed paraffin embedded (FFPE) samples ${ }^{335,336}$. This revived usability of routinely collected FFPE biopsy samples for copy-number analysis immediately broadens the number of available specimens, especially in the case of a disease such as NPC which is very rarely treated with surgery. Given the specific challenges associated with NPC FFPE biopsy specimens, which are often small in size, with significant lymphocytic infiltration, a pilot study was 
undertaken to test the feasibility of analyzing these samples using a SNP array specifically designed by Affymetrix for use with DNA extracted from FFPE specimens $\left(\mathrm{OncoScan}^{\mathrm{TM}}\right)$.

\subsection{Material and Methods}

\subsubsection{Patients and Samples}

FFPE diagnostic blocks from 15 patients diagnosed with NPC between the years of 2002-2008 and treated at Princess Margaret Cancer Center (PM) were collected with approval from the institutional research ethics board (REB). Clinical and follow-up data were collected through chart review; summarized in Table 4-1 for these 15 NPC samples. In addition, 7 FFPE tonsillar specimens with no evidence of malignancy that were archived during the same time period were utilized as reference controls for down stream copy-number calculations. 
Table 4-1. Clinical characteristics of 15 NPC patients analyzed using OncoScan platform

\begin{tabular}{|c|c|}
\hline \multicolumn{2}{|l|}{ Age (years) } \\
\hline median & 52 \\
\hline \multirow[t]{2}{*}{ range } & $17-63$ \\
\hline & Frequency (\%) \\
\hline \multicolumn{2}{|l|}{ Gender } \\
\hline Male & $11(73)$ \\
\hline Female & $4(27)$ \\
\hline \multicolumn{2}{|l|}{ T stage } \\
\hline T1 & $4(27)$ \\
\hline $\mathrm{T} 2$ & $5(33)$ \\
\hline T3 & $4(27)$ \\
\hline T4 & $2(13)$ \\
\hline \multicolumn{2}{|l|}{ N stage } \\
\hline NO & $2(13)$ \\
\hline N1 & $2(13)$ \\
\hline N2 & $8(53)$ \\
\hline N3 & $3(20)$ \\
\hline \multicolumn{2}{|l|}{ TNM Stage } \\
\hline I (\%) & $2(13)$ \\
\hline II (\%) & $2(13)$ \\
\hline III (\%) & $6(40)$ \\
\hline IV (\%) & $5(33)$ \\
\hline Unable to evaluate & none \\
\hline \multicolumn{2}{|l|}{ Treatment } \\
\hline Radiation only & $4(27)$ \\
\hline Radiation + chemo & $11(73)$ \\
\hline
\end{tabular}

5-year \%

$\begin{array}{lc}\text { Survival } & \\ \text { Overall } & 80 \% \\ \text { Disease-Free } & 79 \% \\ \text { Local Relapse-Free } & 91 \% \\ \text { Nodal Relapse-Free } & 100 \% \\ \text { Distant Relapse-Free } & 87 \%\end{array}$




\subsubsection{DNA extraction}

Samples were macro-dissected to ensure that at least $70 \%$ of the material analyzed was malignant epithelium. Genomic DNA was extracted from eight 4- $\mu \mathrm{m}$ sections using the RecoverAll TM Total Nucleic Acid Isolation Kit for FFPE (Ambion) according to the manufacturers instructions. DNA was quantified using the Quant-iT ${ }^{\mathrm{TM}}$ PicoGreen ${ }^{\circledR}$ dsDNA Assay and 20ng of DNA was diluted with water into $25 \mu \mathrm{L}$ volume and sent to Affymetrix for processing.

\subsubsection{SNP Microarray Analysis}

DNA from each sample was analyzed by the Affymetrix Research Service Laboratory (ARSL; Santa Clara, CA, USA) using the OncoScan ${ }^{\mathrm{TM}}$ FFPE Express 2.0 Service. The MIP based assay employed includes $\sim 335,000$ markers for copy number and allele frequency measurement, and 541 markers for somatic mutations frequently observed in cancer ${ }^{336}$. Samples were prepared and analyzed according to manufacture's instructions. Briefly, following overnight probe annealing to template DNA, gaps in hybridized probes are filled by a DNA polymerase reaction in the presence of dNTPs, followed by ligation, removal of unligated probes, linearization of circularized probes, PCR amplification, HaeIII digestion, and denaturing. The fragmented, denatured PCR product is then hybridized to OncoScan microarrays overnight, followed by washing, staining, and finally scanning of the arrays. Output .CEL files from the Affymetrix GeneChip ${ }^{\circledR}$ Scanner are then converted to one Nexus (Biodiscovery) compatible .cbzip file for downstream analysis. 


\subsubsection{Quality control}

Several quality control (QC) metrics were used to determine which samples were likely to provide high quality data. First, sample concentration was re-analyzed on site by the ARSL and all 15 NPC samples were found to have $>20$ ng available DNA by PicoGreen. Second, samples are run on 3\% agarose gels from both the first PCR reaction and the second PCR reaction/HaeIII digestions to ensure high quality products at the correct size. The array intensity values themselves are then assessed for noise using the Median Absolute Pairwise Difference (MAPD) metric. The MAPD algorithm calculates the $\log _{2}$ distance between neighbors for all pairs of probes and then the median is taken across all samples. This represents the typical difference between marker pairs with respect to $\log _{2}$ ratios. The higher the MAPD, the greater the noise between the array of interest and the reference set. Samples with a median MAPD value $>0.6$ are excluded. Finally, Gender is inferred from the ratio of signal from $\mathrm{Y}$ chromosome markers to signal from $\mathrm{X}$ chromosome markers. This provides some degree of sample tracking as well as further evidence of useable data quality.

\subsubsection{Copy-Number Calculation}

All copy-number analysis was performed using Nexus for Affymetrix (Biodiscovery). In order to calculate copy-number values from SNP chip intensities, Nexus first calculates $\log _{2}$ ratios for each probe in experimental samples compared to the mean of the same probe in control samples. Ratios are then corrected for GC content and fragment length using the quadratic correction method in Nexus. Regions with copy-number alterations are then identified using a Hidden Markov Model (HMM) based approach called SNP-FASST2 (Fast Adaptive States Segmentation Technique 2) with the following parameter settings: significance threshold $=5 \times 10^{-7}$, max contiguous probe spacing $=1000 \mathrm{~kb}$, min number of probes per 
segment $=3$, copy-number gain threshold $=0.3$, "high" copy-number gain threshold $=1.2$, copy-number loss threshold $=-0.3$, homozygous copy-number loss threshold $=-1.2$.

In order to identify regions significantly altered within the set of NPC samples, the global frequency statistic approach of the Significance Testing for Aberrant Copy number (STAC) method $^{337}$ was employed with a significance cut off of $p=0.05$ and a frequency cut-off of $\geq 40 \%$ (i.e. at least $6 / 15$ samples).

\subsubsection{Sequence Variant Identification}

Scores were calculated for each somatic mutation assay by taking the $\log _{2}$ ratio for the mutant vs. the reference allele. Scores above 9 were considered valid somatic mutations as per the manufacturer's instructions.

\subsection{Results}

\subsubsection{Quality Control}

All NPC samples passed each step of QC, however only 2 of 7 reference (normal tonsillar) samples passed all QC steps. As a result, an additional frozen sample was added to the analysis by the ARSL to increase the number of reference samples to 3 . The gender was also correctly identified for each sample submitted.

\subsubsection{Copy-Number Events}

With a STAC p-value cut-off of 0.05 and a recurrence cut-off of $40 \%$, a total of 16 regions were found to be significantly amplified or deleted in this small cohort of 15 NPC samples (Figure 4-1, Table 4-2). These significantly altered regions cover 203 genes, including 59 miRNAs. (Table 4-2) 

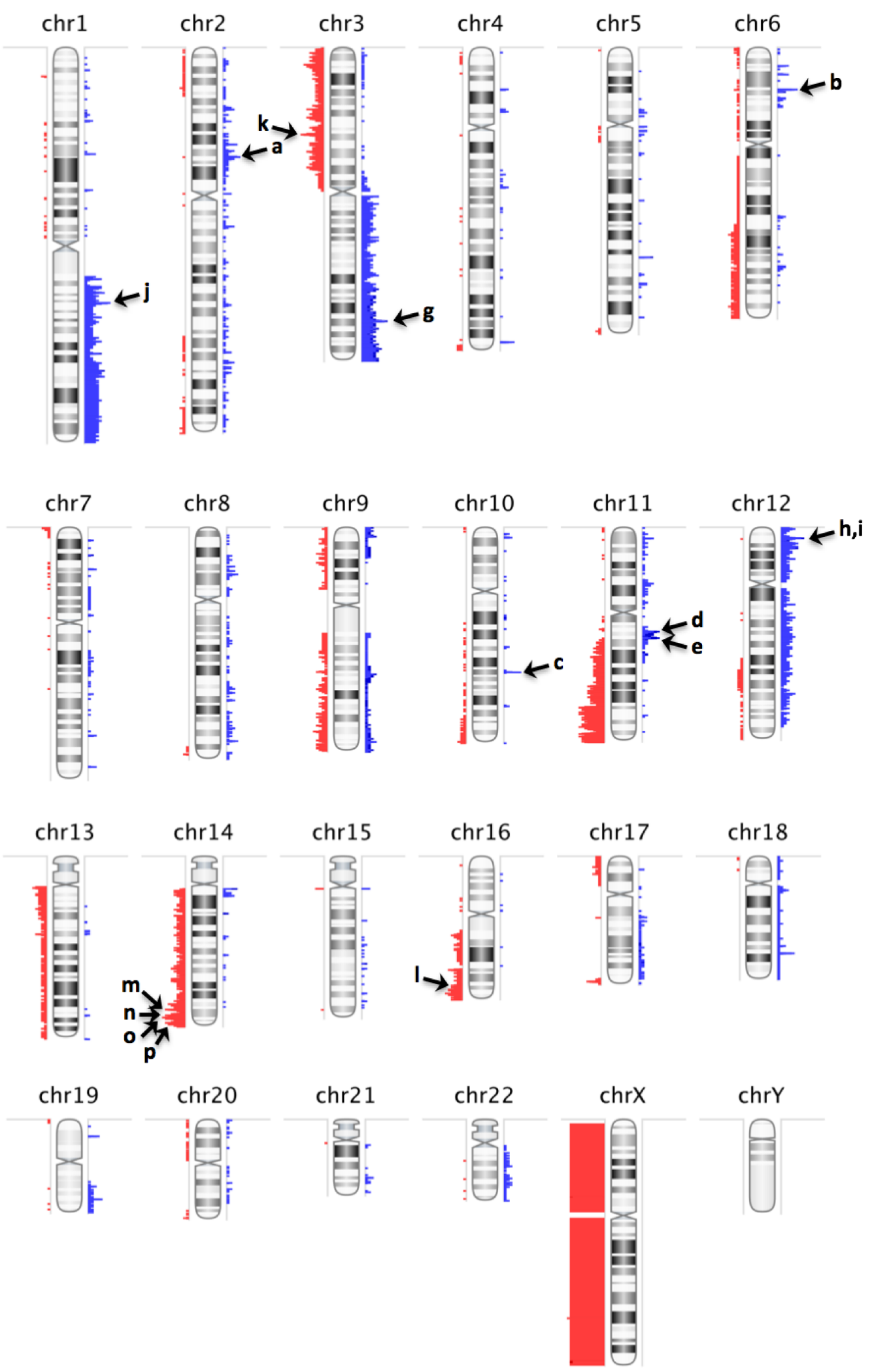

Figure 4-1. Visual representation of genome-wide frequency of CNVs across 15 NPC samples. 
Table 4-2. Genomic regions with significant $(p<0.05)$ CNV events.

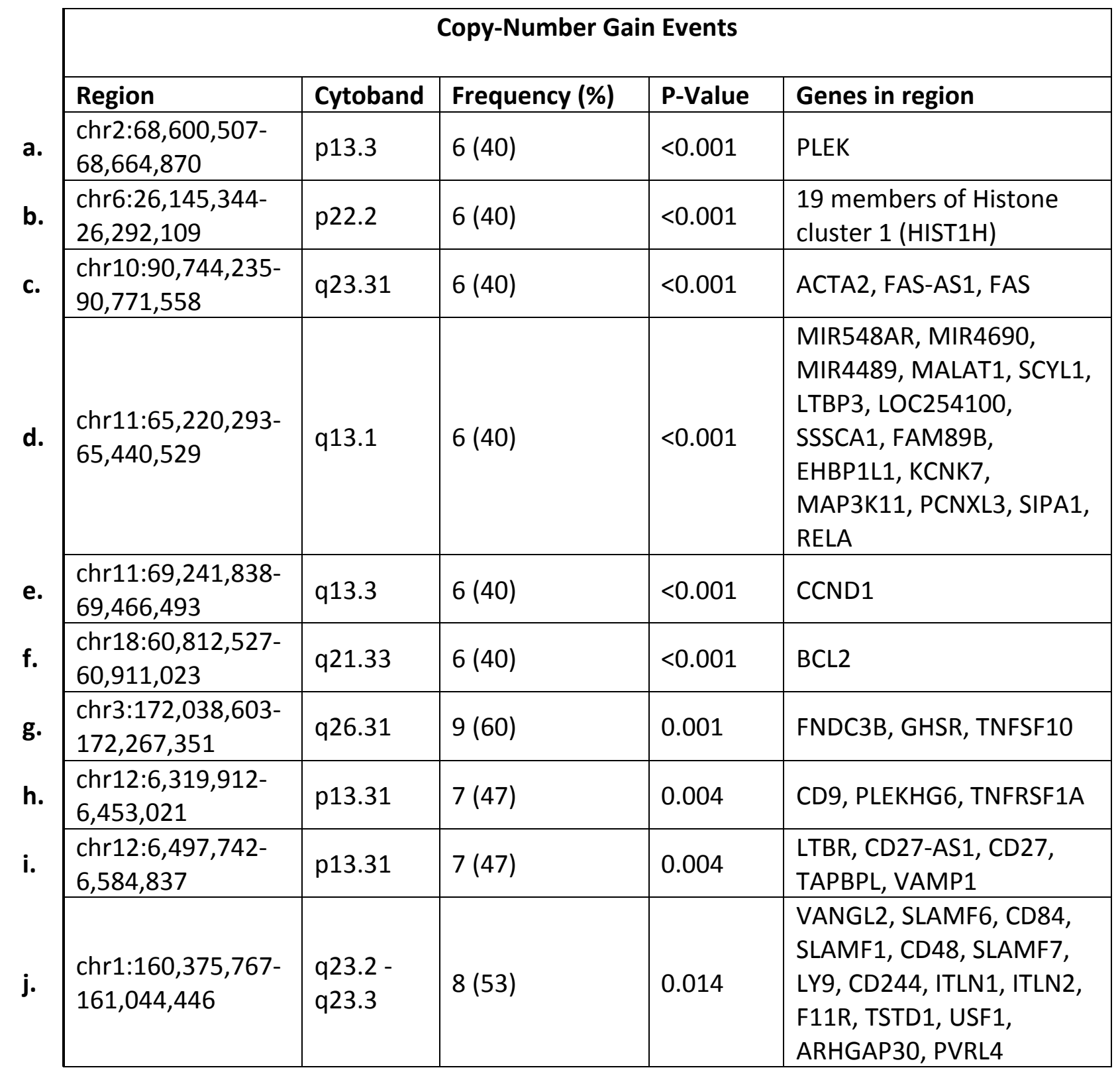


Table 4-2. Continued

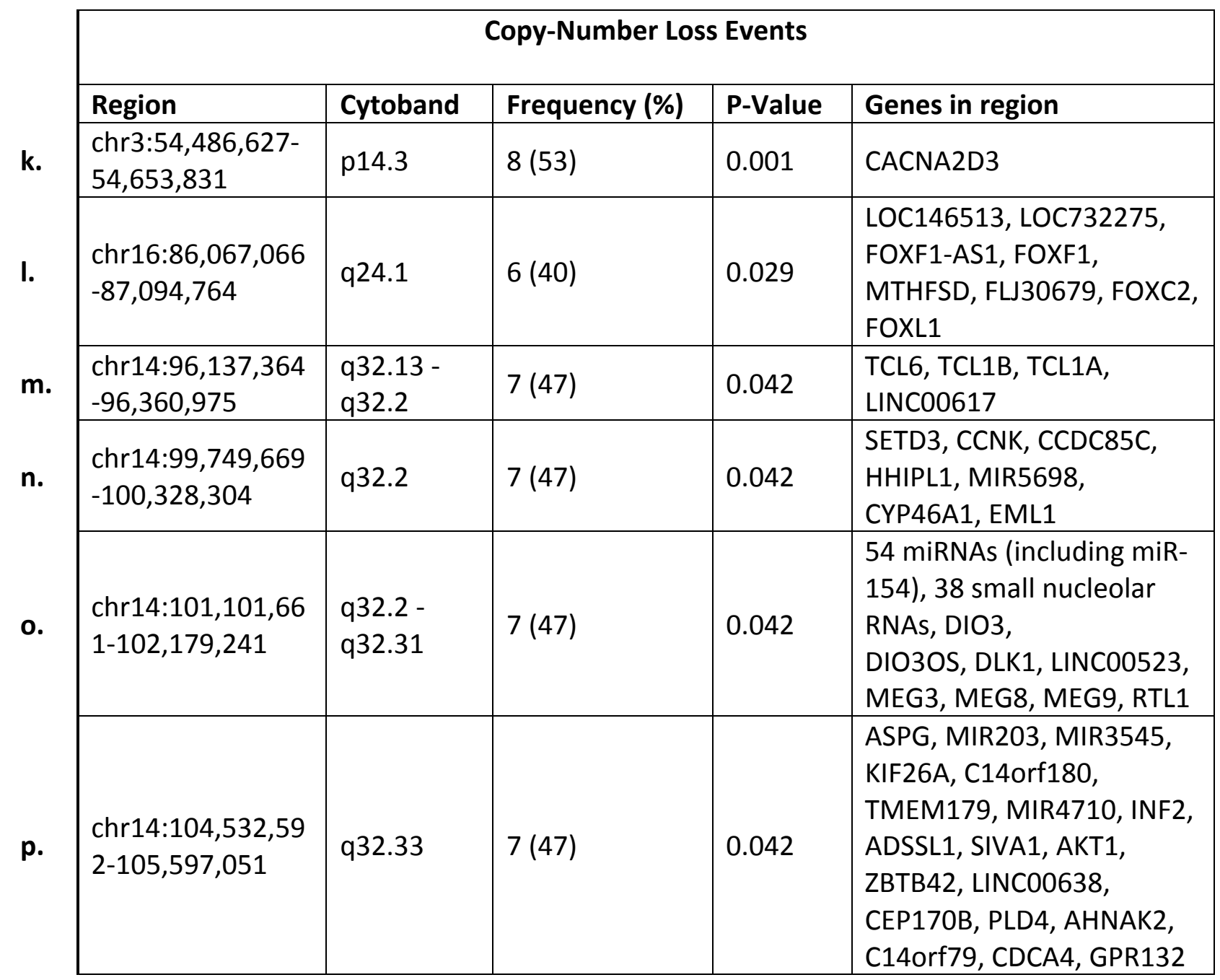




\subsubsection{Sequence Variation Events}

Of the 541 known somatic mutations in cancer assayed, only 2 met the rough somatic mutation score cut-off of 9 in at least one NPC sample. The first, a synonymous variant at residue 561 of fms-related tyrosine kinase 3 (FLT3), was present in 4 of 15 NPC samples. The second, a missense mutation in Ataxia telangiectasia mutated (ATM) resulting in a phenylalanine to leucine amino acid change at residue 858 , was present in only one sample.

\subsection{Discussion and Conclusions}

These data provide us with evidence supporting the feasibility of using the OncoScan (V2) SNP array through Affymetrix's FFPE Express 2.0 Service for copy-number analysis of small FFPE NPC biopsies. However, the application of this technology for identification of sequence variants is less convincing. First of all, only two "somatic mutations" were identified, providing limited evidence either for or against the validity of the OncoScan assay for somatic variant identification. It could be the case that of the 541 mutations included in the assay, only these two were within our 15-sample set, or it could be that the probes were insufficient to detect mutations that were indeed present. Further verification using an alternate technique would be required to fully address this issue.

Of the two sequence variants identified, one was recurrent. This variant was a FLT3 $\mathrm{A}>\mathrm{G}$ transition and was present in 4 of the 15 NPC samples. However, there are three reasons why this variant is unlikely to be of major interest biologically. First, it is synonymous with the reference allele, and therefore unlikely to have a functional impact on the translated protein. Second, this particular variant corresponds with a known polymorphic locus (dbSNP ID: rs34374211) with a global minor allele frequency (MAF) of $12 \%$, and a $16 \%$ frequency in 
Asian populations; not far from the frequency observed herein (4/15, 27\%). Finally, although this variant is included in the catalogue of somatic mutations in cancer (COSMIC), neither of the two studies reporting this variant confirm it is indeed somatic ${ }^{338,339}$. Given these facts, it is likely that this variant is a germline polymorphism, and not related to tumourigenesis in these patients. The other detected sequence variant was a phenylalanine to leucine amino acid change at residue 858 in ATM. This variant also corresponds with a polymorphic locus (dbSNP ID: rs1800056), and while the MAF (0.01\%) is considerably lower than that of the FLT3 mutation, it is similar to the frequency of detection in the current dataset $(1 / 15,0.07 \%)$. In addition, like the FLT3 mutation, neither of the reports cited in this variant's COSMIC entry verify that it is indeed somatic ${ }^{340,341}$. Thus, the inclusion of these probes in the OncoScan assay is likely due to shortcomings in the selection process for these purported "somatic mutation assays".

The somatic mutation assay shortcomings not withstanding; this pilot study did yield some interesting observations from copy-number analysis. In addition to corroborating copynumber changes previously found to be recurrent in the NPC genome, several novel amplified and deleted regions were also identified. Indeed, gains in chromosomes $1 \mathrm{q}, 3 \mathrm{q}$, and $12 \mathrm{p}$ previously discovered by several groups $s^{26,27,342}$ were identified in this pilot study. As well as losses in chromosome $3 \mathrm{p}, 16 \mathrm{q}^{22,342}$, and $14 \mathrm{q}$; which has been previously reported to be lost in up to $85 \%$ of NPC patients ${ }^{22}$. Two of the most significant and most focal recurrently amplified regions identified were located at 11q13.3 and 18q21.33, which encode Cyclin D1 (CCND1) and B-Cell CLL/Lymphoma 2 (BCL-2), respectively. Both CCND1 and BCL-2 are well characterized for their roles in the tumourigenesis of NPC; particularly through promotion of cell growth and survival ${ }^{333}$. While CCND1 is also an established target of chromosomal 
amplification in $\mathrm{NPC}^{43}$, the mechanism of BCL-2 overexpression is not well understood in this disease $^{343}$. The most likely reason for this is a lack in resolution of copy-number strategies previously employed in the analysis of NPC. The majority of early cytogenetic studies in NPC have used relatively crude methods such as Giemsa- $(\mathrm{G})$ banding ${ }^{19}$ qPCR of microsatellite loci, or the higher-resolution aCGH methods. Routine resolution of these techniques ranges from ? 3-10 $\mathrm{Mb}$ (G-banding) to $\sim 1 \mathrm{Mb}(\mathrm{aCGH})$; although modern aCGH platforms have been reported to detection alterations as small as $15 \mathrm{~kb}^{344}$. By contrast, modern SNP arrays can reach a resolution as low as $700 \mathrm{bp} / \mathrm{probe}^{345}$. Moreover, the particular array utilized for this study provides increased coverage of $\sim 200$ known cancer genes to increase resolution in these $\operatorname{areas}^{336}$. In the case of BCL-2, of the 6 samples with an increase in copy-number detected, 5 harboured amplified regions less than 400kb in length (Figure 4-2). These small events would be impossible to detect using G-band and early CGH techniques, and extremely difficult even on the most dense aCGH chips, which could explain the lack of previous evidence for recurrent amplification of this region in NPC, despite the reproducible detection of over-expressed BCL2 in $\mathrm{NPC}^{157,343,346}$.

In conclusion, this study supports the use of MIP-based SNP array techniques, and in particular the OncoScan platform from Affymetrix, to examine genome-wide copy-number changes using small FFPE NPC biopsy samples. This pilot study also validated a number of previously identified recurrent copy-number alterations in NPC, and generated interesting hypotheses for future testing. 
q21.33

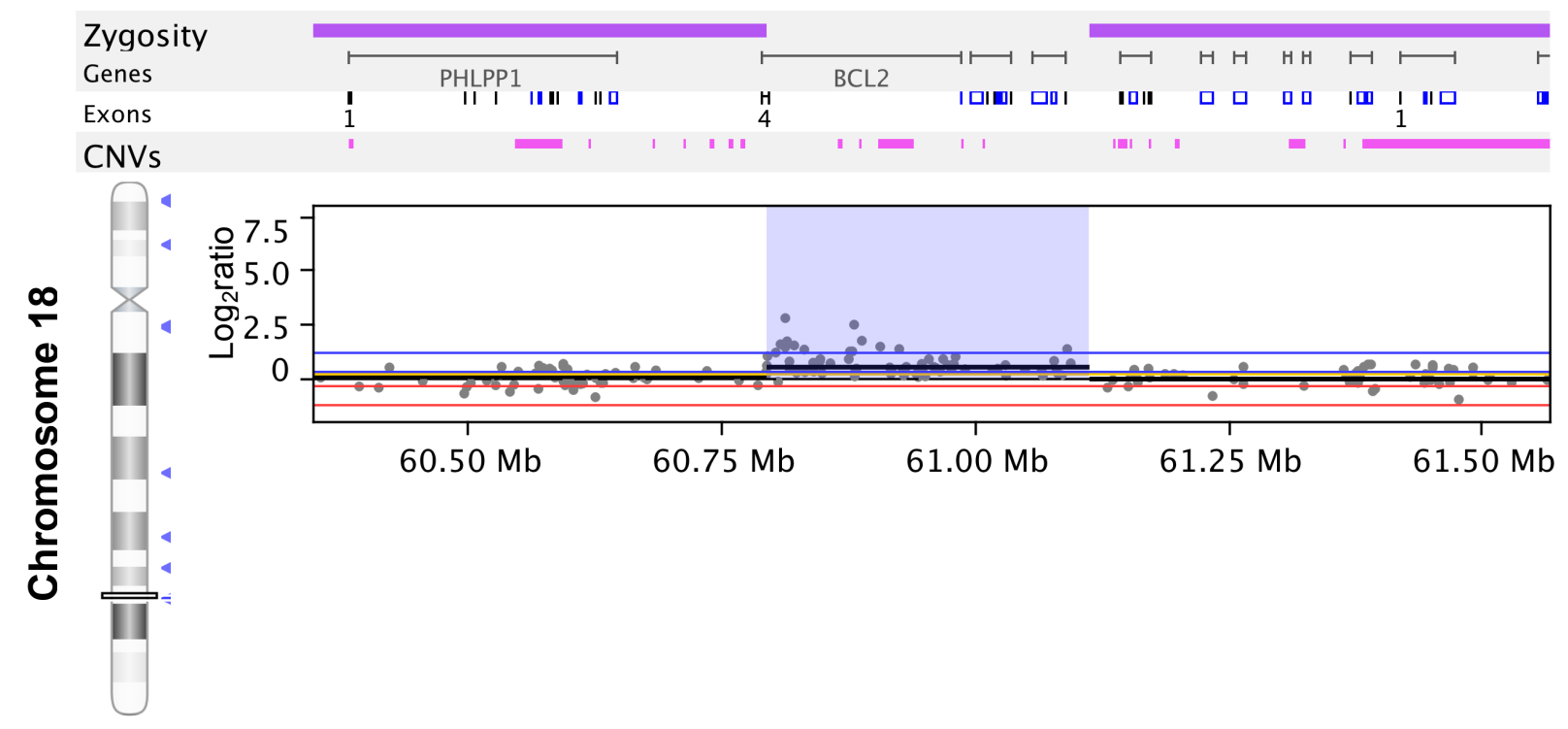

Figure 4-2. Representative sample showing a small (318kb) amplification at Chr18q21.33. Shaded region indicate segments with a median $\log _{2}$ ratio above the threshold (0.3) for copy number gain. 


\section{Chapter 5}

\section{General Discussion, Future Directions, and Conclusions}




\section{General Discussion, Future Directions, and Conclusions}

\subsection{Introduction}

In current clinical practice, the majority of patients who present with NPC are staged using AJCC recommended criteria, and treated with curative RT, with concomitant chemotherapy (5-FU plus a platinum-based compound) in cases of higher stage disease. Advances in radiation delivery methods (i.e. IMRT), and increased adoption of CRT for treatment of NPC patients have resulted in a significant improvement in long-term local control, with 5-year locoregional relapse-free survival rates in excess of 90\%. However, despite these improvements, distant metastasis remains a major cause of death for NPC patients, with up to $17 \%$ of advanced-stage NPC patients experiencing a distant relapse event within 5 years ${ }^{253}$. As a result, distant metastasis is the major cause of NPC related mortality in the current era, underscoring the need for better identification of patients at risk for metastatic disease, as well as developing innovative treatment strategies. Thus, a major focus of this thesis is to address these challenges.

This chapter will discuss the findings expounded in this thesis, put forth corroborating and enhancing observations, as well as outline future studies that will be required to propel these discoveries into clinical use to the ultimate benefit of future NPC patients.

\subsection{General Discussion}

\subsubsection{The role of microRNA-375 and metadherin in NPC}

Chapter 2 described our identification of MTDH as an important target of miR-375 in the context of NPC as well as HNSCC. Our study demonstrated dysregulation of both miR-375 and MTDH in primary NPC and HNSCC specimens, as well as the functional impact of these 
alterations in HNC models, including one potential mechanism via activation of the PI3K-Akt pathway. We also identified a potential prognostic role for MTDH in NPC wherein overexpression of MTDH mRNA was associated with an increased risk of distant relapse. The validity of our report has been subsequently corroborated to varying degrees in ensuing studies performed by others, which will be reviewed below.

\subsubsection{Scientific impact and subsequent research}

Since the publication of our study "Significance of dysregulated metadherin and microRNA-375 in head and neck cancer" in October $2011^{315}$, it has been cited in 23 articles, including both reports of original research and scientific reviews. To the best of our knowledge, our study was the first to report a role for MTDH in NPC, and the second, preceded only by $\sim 3$ months ${ }^{275}$, in head and neck cancer (HNC). Intriguingly, simultaneous to our own study, Nohata et $a l^{275}$ also identified MTDH as a target of miR-375. Subsequently, this same relationship has been identified in hepatocellular carcinoma ${ }^{347}$, breast cancer ${ }^{348}$, and esophageal squamous cell carcinoma ${ }^{349}$ models. Moreover, since the publication of our study, miR-375 and MTDH have been corroborated in numerous cancer types as either a tumour suppressor, or an oncogene, in 84 and 94 publications, respectively. Notably, several of these publications also reproduced our own findings in that MTDH promoted a malignant phenotype by activating the PI3K-Akt pathway ${ }^{350-354}$. Furthermore, many studies have corroborated the role of high MTDH expression as an unfavorable prognostic marker in multiple malignancies, including

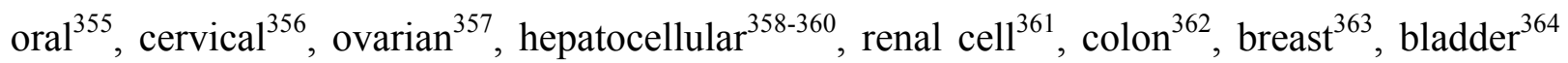
and non-small cell lung cancer ${ }^{365}$; all strong indicators that MTDH is likely to be an important mediator of human cancer development and progression. 


\subsubsection{MiRNA Signature of Distant Metastasis in NPC}

In Chapter 3, I described a 4-miRNA signature associated with risk of distant metastasis in NPC. This signature was generated in a training set of 125 NPC samples using a penalized $\mathrm{CoxPH}$ method for variable selection and coefficient shrinkage. Using the coefficients obtained from the model, the following equation was derived to calculate a risk score for each patient: $R S=\operatorname{miR}-449 b * 0.28+\operatorname{miR}-154 * 0.417-\operatorname{miR}-140.5 p * 0.653-\operatorname{miR}-34 c .5 p * 0.311$. When dichotomized to high ( $\mathrm{RS} \geq$ median) $v s$. low ( $\mathrm{RS} \leq$ median) risk groups, those patients in the high risk group had a significantly increased risk of developing distant metastasis compared to

the low risk group $\left(\mathrm{HR}=8.25 ; \mathrm{p}=8.0 \times 10^{-4}\right)$. This signature was then validated in the second independent set of 121 NPC patients $(\mathrm{HR}=3.2 ; \mathrm{p}=0.014)$. Furthermore, a multivariate analysis demonstrated the strong independent prognostic value of this 4-miRNA signature, which was able to add prognostic value to nodal involvement; the best current predictor of distant metastasis.

\subsubsection{Additional Analysis}

Given that Chapters 2 and 3 both reported on the identification of expression signatures associated with distant metastasis in NPC, I was interested to determine if combining the three variables consistently associated with distant metastasis in NPC (MTDH expression, our 4miRNA signature, and N-stage) in one model could further improve prognostic ability. When 88 samples with both MTDH and 4-miRNA risk-score data were used, a CoxPH model with MTDH, 4-miRNA signature and N-stage as variables generated the following equation:

Risk Score $=(4-m i R N A$ risk group $) * 5.08+($ MTDH risk group $) * 6.81+(\mathrm{N}-$ stage $) * 1.71$ Where risk groups were defined as 0 for low risk and 1 for high risk, and N-stage was 
numerical 0-3 for N0-N3. Not surprisingly, when samples were dichotomized by median Risk Score, patients in the high-risk group had a significantly higher risk of distant metastasis $(\mathrm{HR}=$ 12; $\mathrm{p}=0.002$; Figure 5-1F). Most intrigueingly, this combined model appeared to perform the best across multiple time points compared to each signature alone or individually combined with N-stage (Figure 5-1G,H). Interpretation of these results is relatively limited due to the small sample size, and relatively few metastasis events (13), therefore further studies should be undertaken in independent cohorts of patients to determine the full utility of this combined signature. 
A

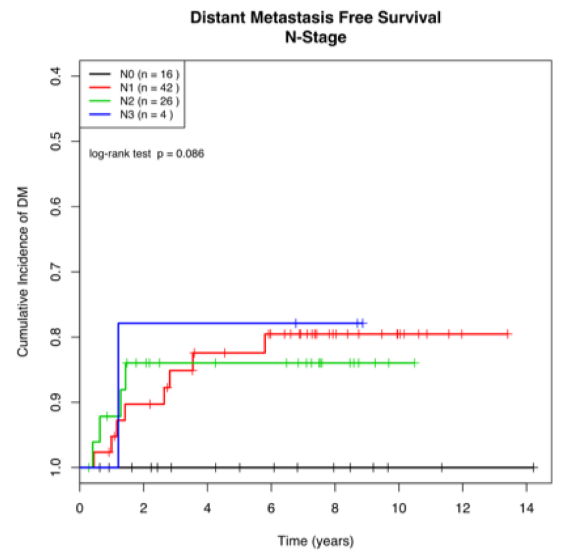

D

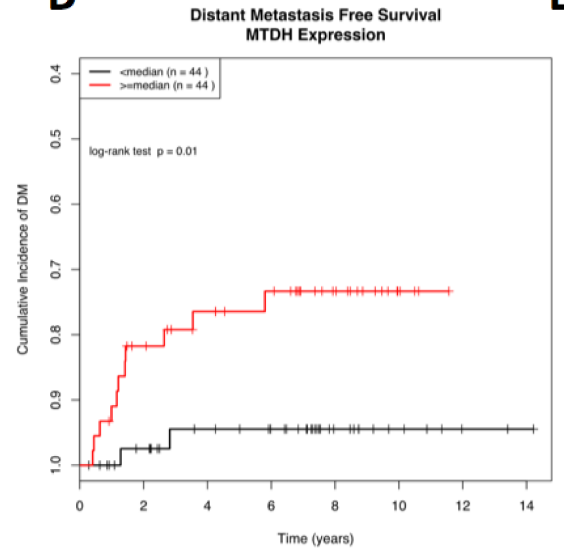

G

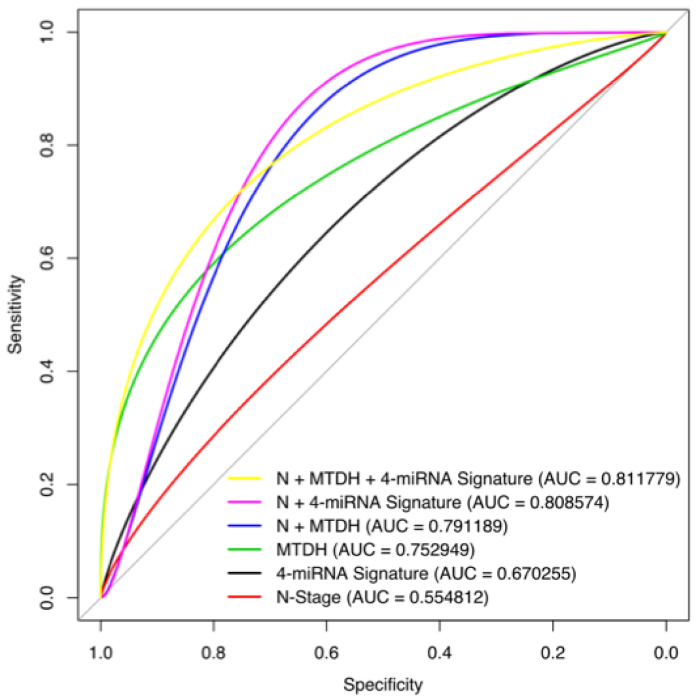

B

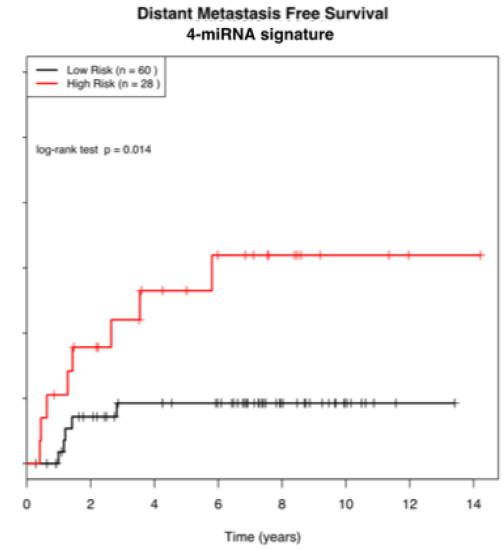

E

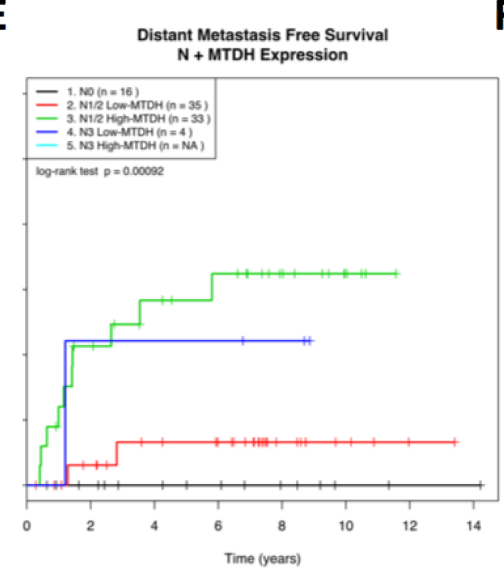

C

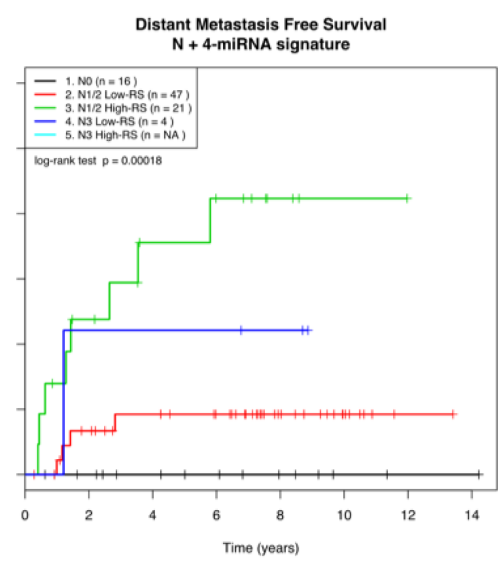

$\mathbf{F}$

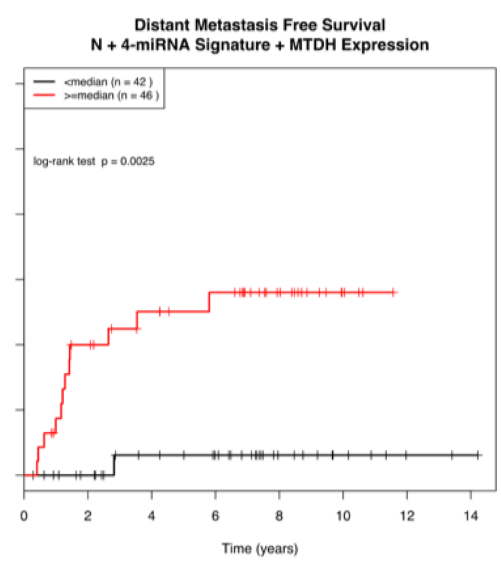

H

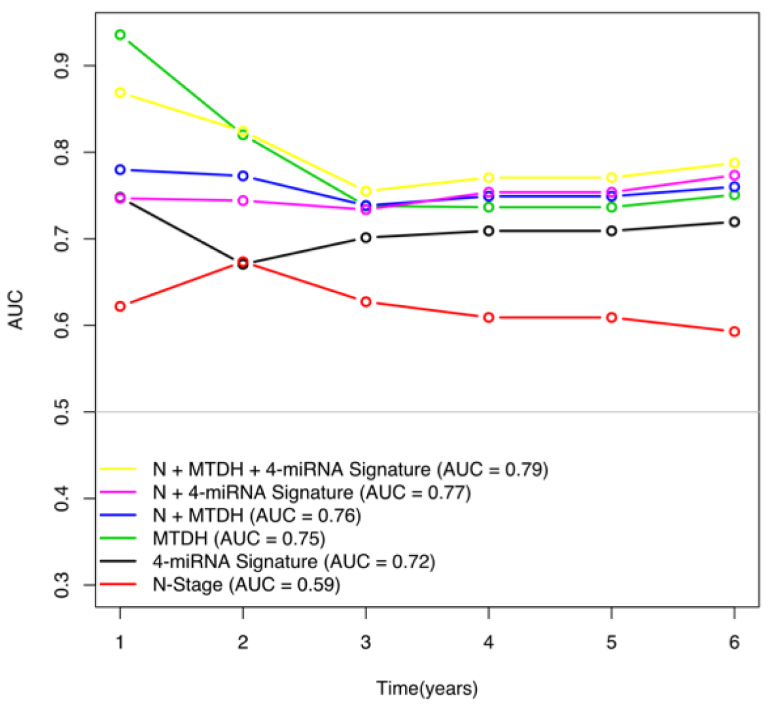

Figure 5-1. Comparison of the prognostic ability of MTDH, the 4-miRNA signature and $\mathrm{N}$-stage; combined and individually.

88 samples from the previously described cohort of NPC patients diagnosed between 1993 and 2000 were used to identify the optimal signature of distant metastasis risk in NPC. 


\subsubsection{Genomic Characterization of NPC Samples}

Although brief, our pilot study to characterize the genome of 15 FFPE NPC biopsy samples provided two major insights: 1) that CNA profiling of small FFPE NPC biopsies is technically feasible using a MIP-based technology and 2) that this technique can be used to confirm known and identify novel CNA events in NPC. To the best of our knowledge, there is no published article to date reporting the use of an MIP assay for CNA profiling in NPC samples. Moreover, the results suggest that the superior resolution of this platform compared with methods previously used for CNA analysis in NPC can provide novel insights into the genomic landscape of NPC. In particular, the observation of frequent amplification of BCL-2 is especially intriguing. While BCL-2 is a well established oncogene which is consistently overexpressed in $\mathrm{NPC}^{157,343,346}$, and chromosomal amplification is a mechanism of Bcl-2 overexpression in B-cell lymphomas ${ }^{366}$, recurrent amplification of this genomic region has yet to be described in NPC. Indeed, it may well be the increased resolution afforded by this modern technique that enabled the detection of these relatively small $(\sim 400 \mathrm{~kb})$ regions of amplification, where lower resolution methods had failed. Thus, MIP-based arrays are a feasible and informative method to interrogate archival FFPE NPC biopsy specimens.

\subsection{Future Directions}

\subsubsection{The role of microRNA-375 and metadherin in NPC}

\subsubsection{Clinical Implementation}

The findings in our 2011 study have two major potential clinical applications: 1) miR375, MTDH or downstream/upstream effectors are potential targets of novel therapeutics; and

2) MTDH expression could serve as a prognostic/predictive biomarker in NPC. However, in 
order to translate these findings into clinically useful tools, additional investigation must be undertaken.

We and others have put forth strong cases supporting miR-375 as a tumour suppressor, and MTDH as an oncogene in HNC. While our report remains the only study to demonstrate a role for MTDH or miR-375 directly in NPC, the applicability in this disease is perhaps the most compelling given the strong correlation between MTDH expression and distant metastasis. Two obvious options present themselves for translation of these findings into therapeutic approaches: replacement of miR-375 or knock-down/inhibition of MTDH in NPC patients. While much headway has been made towards so-called "gene-therapy" for cancer treatment, ectopic replacement of tumour suppressor genes or RNA-interference mediated knock-down of oncogenes are still in the pre-clinical/early clinical stages of evaluation, and continued advancements in delivery methods will be necessary before such techniques can be applicable in the clinical arena ${ }^{367}$. As such, small molecule inhibitors of MTDH or downstream signaling pathway members might be more viable options. To date, no small molecule inhibitors of MTDH have been reported, and the development of such chemicals might be difficult due to a lack of traditionally "druggable" features such as a kinase domain or a known ligand ${ }^{368,369}$. Thus, the current state of the art dictates that inhibition of MTDH signaling pathway members might be the only imminently available approach. Our study demonstrated that MTDH knockdown in NPC cells resulted in a significant reduction in phospho-Akt (Figure 2-11A), and indeed, several pre-clinical studies have shown efficacy of several PI3K, mTOR, and dual inhibitors in this disease ${ }^{370-373}$. However, given that PI3K is upstream of Akt, MTDH overexpression in NPC could circumvent the inhibition of this receptor via alternative phosphorylation of Akt. In contrast, mTOR inhibitors primarily inhibit the mTORC1 complex 
which is downstream of $\mathrm{Akt}^{374}$, perhaps making this a more viable candidate for targeted therapies in MTDH over-expressing patients. Thus, MTDH expression could potentially be used as a predictive biomarker for future clinical trials employing PI3K-mTOR-Akt pathway inhibitors.

For MTDH expression to be a clinically useful prognostic or predictive biomarker, an analytical method for detection must first be validated. Our study reported the use of qRT-PCR in RNA extracted from FFPE biopsy samples; however, this technique is not routinely applied in pathology departments, hence would be difficult to implement broadly. MTDH expression measured by IHC staining, a much more ubiquitously utilized diagnostic technique, has been

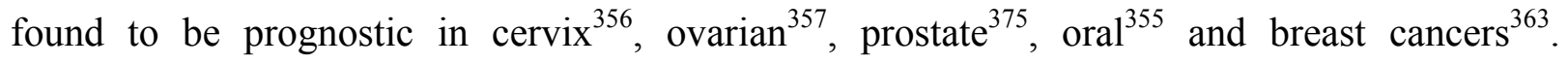
However, our initial MTDH IHC staining results in NPC samples were skewed towards high intensity staining, suggesting saturation in the signal (Figure 2-5C\&D). Consequently, IHC staining did not correlate well with the qRT-PCR data, nor were these values associated with metastasis (data not shown). It is possible that particularly subtle MTDH expression variation in NPC excludes IHC as an appropriate analytical technique in this disease, however it is likely that optimization of the staining protocol could remedy this challenge.

\subsubsection{Biological Investigation}

In addition to overcoming the challenges of implementing our discoveries in the clinic, we are also interested in further elucidating the biological functions of miR-375 and MTDH within NPC cells, which still remains largely unknown.

First, our preliminary data suggests that hypermethylation of the miR-375 genomic region plays a role in its suppression, however the mechanism by which this region is 
methylated as well as a direct role in transcription of these regions has not been established. There is evidence to suggest that LMP1 is capable of increasing the expression of several DNMTs, as such, over-expression and/or knock-down of LMP1 expression and subsequent miR-375 expression and CpG island methylation analysis would provide further evidence that LMP1 mediated methylation is indeed responsible for the wide-spread under-expression of miR-375 observed in NPC samples.

Second, additional targets of miR-375 may very well also be important to NPC development, and exploration of these in in vitro and in vivo models, similar to those detailed in Chapter 2, could provide further insight into the biology of NPC. Similarly, while we have established that at least one function of $\mathrm{MTDH}$ in NPC cells is the activation PI3K/Akt signaling, MTDH is also known to target a number of additional pro-growth and anti-apoptotic pathways in a variety of cancers. Rescue experiments using constitutively active Akt in conjunction with MTDH knockdown could provide evidence that this is the pathway required for MTDH mediated survival signaling in NPC. In contrast, probing other pathways such as

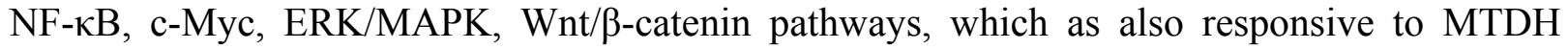

mediated activation $^{376}$, could determine if more than just the PI3K/Akt pathway is mediating MTDH's survival, growth, migration and invasion promoting effects in NPC.

\subsubsection{MiRNA Signature of Distant Metastasis in NPC}

\subsubsection{Clinical Implementation}

In order to fully elucidate the clinical utility of the prognostic signatures presented herein, and to determine the optimal signature for prediction of distant metastasis in NPC, examination in an additional cohort of patients would be of immense benefit. Furthermore, the 
treatment homogeneity and randomized nature of samples collected prospectively as part of a randomized clinical trial would improve the scientific validity and clinical applicability. Hence, we have established a collaboration with the Pamela Youde Nethersole Eastern Hospital in Hong Kong who have agreed to provide an additional 189 FFPE NPC blocks from participants in an RCT, whereby NPC patients with locally-advanced disease were randomized to CRT vs.

RT alone ${ }^{14,377}$. These samples will be analyzed in a similar manner to that described above, with qRT-PCR used to measure the 4 miRNAs from our signature, the 5 miRNAs from Liu et al.'s recently published signature ${ }^{183}$, as well as MTDH and housekeeping controls. Through statistical analysis, we will then attempt to design an optimized signature associated with distant metastasis. The randomized nature of this study will also allow us to draw important conclusions regarding the ability of these signatures to predict success/failure of each treatment modality (CRT vs. RT alone).

\subsubsection{Biological Investigation}

Preliminary pathway enrichment analysis presented in Chapter 3 indicated that validated targets of the members of our 4-miRNA signature converged on the cell-cycle control pathway. However, extensive functional characterization is still required in order to establish if and how these miRNAs regulate the oncogenic phenotype in NPC. These functional experiments will be conducted in a similar fashion to those described in Chapter 2 regarding the characterization of miR-375. Briefly, we will first establish the phenotypic impact of over or under-expression of these miRNAs in NPC cell lines in vitro. Targets of functionally relevant miRNAs will then be identified using a combination of in silico, and in vitro approaches. Candidate mRNA targets will be confirmed using the luciferase reporter assay, followed by phenotypic (e.g. cell cycle, 
migration, invasion), and biochemical (Western blotting, qRT-PCR for mRNA level) analyses in vitro, and in mouse xenograft models of NPC. One limitation is a lack of a robust mouse model of metastatic EBV-positive NPC. The only consistently EBV positive cell line, C666-1 does not readily metastasize from the widely used intra-muscular or subcutaneous xenograft sites. A few studies have reported alternative methods such as intravenous or intra-hepatic injections; however these clearly do not accurately recapitulate true NPC metastasis ${ }^{206,378}$. One group has, however, successfully established an orthotopic model of NPC wherein C666-1 cells are injected directly into the nasopharynx of NOD/SCID/gamma mice, with the subsequent development of distant metastases to the bone, lung, and liver ${ }^{373}$. While this is likely to be a technically challenging technique, establishment of this model in our own laboratory would be of great benefit for further investigation into the functional impact of the various miRNAs and their targets of putative importance in metastatic NPC. It will also provide a valuable NPC model for pre-clinical evaluation of novel targeted therapies.

\subsubsection{Genomic Characterization of NPC Samples}

The work described in Chapter 4 was a pilot study to determine the feasibility of obtaining genome-wide copy-number, and potentially mutation data from FFPE NPC biopsies using a MIP-based approach. Given the positive results of this study, work is currently underway to similarly analyze an additional 200 NPC samples as part of an integrated DNA, RNA, and protein profiling effort by our group at the Princess Margaret Cancer Centre. The data generated will be used to both provide useful insight into the biology of NPC as well as to generate a prognostic model which integrates the relevant data types to optimize predictive value. In the short term, confirmation of BCL-2 amplification in a larger cohort of NPC 
samples would provide further evidence that this novel observation is indeed an important event in NPC development.

\subsection{Conclusion}

The main focus of the studies outlined within this thesis aimed to provide an understanding of NPC biology and clinical behavior through the lens of miRNAs. Chapter 2 focused primarily on the biological impact of the robust down-regulation of miR-375 observed in NPC. These studies led to the identification of MTDH, an emerging oncogene in human cancers, as a potentially important promoter of NPC progression and metastasis. Armed with these data, future studies may determine strategies to target this gene and its affected pathways, or exploit it as a marker of aggressive disease. In Chapter 3, we identified and validated a miRNA signature associated with distant metastasis in NPC patients. This signature could better enable physicians to tailor treatments based on an individual patient's prognosis; those patients at low risk of distant failure could potentially be spared the toxicity of aggressive treatments, while providing currently employed aggressive approaches or novel targeted therapies to those patients who could benefit from them. Finally, in Chapter 4 we took a somewhat different approach; utilizing copy-number data to identify potentially important genomic aberrations in NPC samples. Overall, these studies bring us closer to the ever-more attainable goal of truly personalized treatment for NPC patients. 


\section{References}

1. Chang ET, Adami HO: The enigmatic epidemiology of nasopharyngeal carcinoma. Cancer Epidemiol Biomarkers Prev 15:1765-77, 2006

2. Deyrup AT: Epstein-Barr virus-associated epithelial and mesenchymal neoplasms. Hum Pathol 39:473-83, 2008

3.Xue WQ, Qin HD, Ruan HL, et al: Quantitative association of tobacco smoking with the risk of nasopharyngeal carcinoma: a comprehensive meta-analysis of studies conducted between 1979 and 2011. Am J Epidemiol 178:325-38, 2013

4. Yu MC, Yuan JM: Epidemiology of nasopharyngeal carcinoma. Semin Cancer Biol $12: 421-9,2002$

5. Gallicchio L, Matanoski G, Tao XG, et al: Adulthood consumption of preserved and nonpreserved vegetables and the risk of nasopharyngeal carcinoma: a systematic review. Int J Cancer 119:1125-35, 2006

6. Poirier S, Bouvier G, Malaveille C, et al: Volatile nitrosamine levels and genotoxicity of food samples from high-risk areas for nasopharyngeal carcinoma before and after nitrosation. Int J Cancer 44:1088-94, 1989

7. Glade MJ: Food, nutrition, and the prevention of cancer: a global perspective. American Institute for Cancer Research/World Cancer Research Fund, American Institute for Cancer Research, 1997. Nutrition 15:523-6, 1999

8. Caponigro F, Longo F, Ionna F, et al: Treatment approaches to nasopharyngeal carcinoma: a review. Anticancer Drugs 21:471-7, 2010

9. Guigay J: Advances in nasopharyngeal carcinoma. Curr Opin Oncol 20:264-9, 2008

10. Chan AT, Hsu MM, Goh BC, et al: Multicenter, phase II study of cetuximab in combination with carboplatin in patients with recurrent or metastatic nasopharyngeal carcinoma. J Clin Oncol 23:3568-76, 2005

11. He XY, Hu CS, Ying HM, et al: Paclitaxel with cisplatin in concurrent chemoradiotherapy for locally advanced nasopharyngeal carcinoma. Eur Arch Otorhinolaryngol 267:773-8, 2010

12. Al-Sarraf M, LeBlanc M, Giri PG, et al: Chemoradiotherapy versus radiotherapy in patients with advanced nasopharyngeal cancer: phase III randomized Intergroup study 0099. J Clin Oncol 16:1310-7, 1998

13. Lu H, Peng L, Yuan X, et al: Concurrent chemoradiotherapy in locally advanced nasopharyngeal carcinoma: a treatment paradigm also applicable to patients in Southeast Asia. Cancer Treat Rev 35:345-53, 2009 
14. Lee AW, Tung SY, Chan AT, et al: Preliminary results of a randomized study (NPC9902 Trial) on therapeutic gain by concurrent chemotherapy and/or accelerated fractionation for locally advanced nasopharyngeal carcinoma. Int J Radiat Oncol Biol Phys $66: 142-51,2006$

15. Zhang L, Zhao C, Ghimire B, et al: The role of concurrent chemoradiotherapy in the treatment of locoregionally advanced nasopharyngeal carcinoma among endemic population: a meta-analysis of the phase III randomized trials. BMC Cancer 10:558, 2010

16. Lo KW, To KF, Huang DP: Focus on nasopharyngeal carcinoma. Cancer Cell 5:423-8, 2004

17. Chan AS, To KF, Lo KW, et al: High frequency of chromosome $3 p$ deletion in histologically normal nasopharyngeal epithelia from southern Chinese. Cancer Res 60:5365-70, 2000

18. Chan AS, To KF, Lo KW, et al: Frequent chromosome 9p losses in histologically normal nasopharyngeal epithelia from southern Chinese. Int J Cancer 102:300-3, 2002

19. Huang DP, Ho JH, Chan WK, et al: Cytogenetics of undifferentiated nasopharyngeal carcinoma xenografts from southern Chinese. Int J Cancer 43:936-9, 1989

20. Mitelman F, Mark-Vendel E, Mineur A, et al: A 3q+ marker chromosome in EBVcarrying nasopharyngeal carcinomas. Int J Cancer 32:651-5, 1983

21. Waghray M, Parhar RS, Taibah K, et al: Rearrangements of chromosome arm 3q in poorly differentiated nasopharyngeal carcinoma. Genes Chromosomes Cancer 4:326-30, 1992

22. Lo KW, Teo PM, Hui AB, et al: High resolution allelotype of microdissected primary nasopharyngeal carcinoma. Cancer Res 60:3348-53, 2000

23. Hui AB, Lo KW, Leung SF, et al: Loss of heterozygosity on the long arm of chromosome 11 in nasopharyngeal carcinoma. Cancer Res 56:3225-9, 1996

24. Tsang YS, Lo KW, Leung SF, et al: Two distinct regions of deletion on chromosome 13q in primary nasopharyngeal carcinoma. Int J Cancer 83:305-8, 1999

25. Wong N, Hui AB, Fan B, et al: Molecular cytogenetic characterization of nasopharyngeal carcinoma cell lines and xenografts by comparative genomic hybridization and spectral karyotyping. Cancer Genet Cytogenet 140:124-32, 2003

26. Hui AB, Lo KW, Leung SF, et al: Detection of recurrent chromosomal gains and losses in primary nasopharyngeal carcinoma by comparative genomic hybridisation. Int J Cancer 82:498-503, 1999 
27. Chen YJ, Ko JY, Chen PJ, et al: Chromosomal aberrations in nasopharyngeal carcinoma analyzed by comparative genomic hybridization. Genes Chromosomes Cancer 25:169-75, 1999

28. Huang DP, Lo KW, van Hasselt CA, et al: A region of homozygous deletion on chromosome 9p21-22 in primary nasopharyngeal carcinoma. Cancer Res 54:4003-6, 1994

29. Lo KW, Cheung ST, Leung SF, et al: Hypermethylation of the p16 gene in nasopharyngeal carcinoma. Cancer Res 56:2721-5, 1996

30. Kwong J, Lo KW, To KF, et al: Promoter hypermethylation of multiple genes in nasopharyngeal carcinoma. Clin Cancer Res 8:131-7, 2002

31. Wang GL, Lo KW, Tsang KS, et al: Inhibiting tumorigenic potential by restoration of p16 in nasopharyngeal carcinoma. Br J Cancer 81:1122-6, 1999

32. Li HM, Man C, Jin Y, et al: Molecular and cytogenetic changes involved in the immortalization of nasopharyngeal epithelial cells by telomerase. Int J Cancer 119:1567-76, 2006

33. Lo K, Tsao S, Leung S, et al: Detailed deletion mapping on the short arm of chromosome-3 in nasopharyngeal carcinomas. Int J Oncol 4:1359-64, 1994

34. Lo KW, Kwong J, Hui AB, et al: High frequency of promoter hypermethylation of RASSF1A in nasopharyngeal carcinoma. Cancer Res 61:3877-81, 2001

35. Dammann R, Li C, Yoon JH, et al: Epigenetic inactivation of a RAS association domain family protein from the lung tumour suppressor locus 3p21.3. Nat Genet 25:315-9, 2000

36. Fernandes MS, Carneiro F, Oliveira C, et al: Colorectal cancer and RASSF family--a special emphasis on RASSF1A. Int J Cancer 132:251-8, 2013

37. Chow LS, Lo KW, Kwong J, et al: RASSF1A is a target tumor suppressor from $3 \mathrm{p} 21.3$ in nasopharyngeal carcinoma. Int J Cancer 109:839-47, 2004

38. Chow LS, Lam CW, Chan SY, et al: Identification of RASSF1A modulated genes in nasopharyngeal carcinoma. Oncogene 25:310-6, 2006

39. Song MS, Song SJ, Ayad NG, et al: The tumour suppressor RASSF1A regulates mitosis by inhibiting the APC-Cdc20 complex. Nat Cell Biol 6:129-37, 2004

40. Song SJ, Song MS, Kim SJ, et al: Aurora A regulates prometaphase progression by inhibiting the ability of RASSF1A to suppress APC-Cdc20 activity. Cancer Res 69:231423, 2009

41. van der Weyden L, Tachibana KK, Gonzalez MA, et al: The RASSF1A isoform of RASSF1 promotes microtubule stability and suppresses tumorigenesis. Mol Cell Biol 25:8356-67, 2005 
42. Man C, Rosa J, Lee LT, et al: Latent membrane protein 1 suppresses RASSF1A expression, disrupts microtubule structures and induces chromosomal aberrations in human epithelial cells. Oncogene 26:3069-80, 2007

43. Hui AB, Or YY, Takano H, et al: Array-based comparative genomic hybridization analysis identified cyclin D1 as a target oncogene at 11q13.3 in nasopharyngeal carcinoma. Cancer Res 65:8125-33, 2005

44. Or YY, Chung GT, To KF, et al: Identification of a novel 12p13.3 amplicon in nasopharyngeal carcinoma. J Pathol 220:97-107, 2010

45. Or YY, Hui AB, To KF, et al: PIK3CA mutations in nasopharyngeal carcinoma. Int J Cancer 118:1065-7, 2006

46. Morrison JA, Gulley ML, Pathmanathan R, et al: Differential signaling pathways are activated in the Epstein-Barr virus-associated malignancies nasopharyngeal carcinoma and Hodgkin lymphoma. Cancer Res 64:5251-60, 2004

47. Spruck CH, 3rd, Tsai YC, Huang DP, et al: Absence of p53 gene mutations in primary nasopharyngeal carcinomas. Cancer Res 52:4787-90, 1992

48. Sun Y, Hegamyer G, Cheng YJ, et al: An infrequent point mutation of the p53 gene in human nasopharyngeal carcinoma. Proc Natl Acad Sci U S A 89:6516-20, 1992

49. Effert P, McCoy R, Abdel-Hamid M, et al: Alterations of the p53 gene in nasopharyngeal carcinoma. J Virol 66:3768-75, 1992

50. Chung GT, Lou WP, Chow C, et al: Constitutive activation of distinct NF-kappaB signals in EBV-associated nasopharyngeal carcinoma. J Pathol 231:311-22, 2013

51. Chou CC, Chou MJ, Tzen CY: PIK3CA mutation occurs in nasopharyngeal carcinoma but does not significantly influence the disease-specific survival. Med Oncol 26:322-6, 2009

52. Wei MX, Ooka T: A transforming function of the BARF1 gene encoded by EpsteinBarr virus. EMBO J 8:2897-903, 1989

53. Decaussin G, Sbih-Lammali F, de Turenne-Tessier M, et al: Expression of BARF1 gene encoded by Epstein-Barr virus in nasopharyngeal carcinoma biopsies. Cancer Res 60:55848,2000

54. Frappier L: Role of EBNA1 in NPC tumourigenesis. Semin Cancer Biol 22:154-61, 2012

55. Li HP, Chang YS: Epstein-Barr virus latent membrane protein 1: structure and functions. J Biomed Sci 10:490-504, 2003 
56. Zheng H, Li LL, Hu DS, et al: Role of Epstein-Barr virus encoded latent membrane protein 1 in the carcinogenesis of nasopharyngeal carcinoma. Cell Mol Immunol 4:185-96, 2007

57. Yoshizaki T, Kondo S, Wakisaka N, et al: Pathogenic role of Epstein-Barr virus latent membrane protein-1 in the development of nasopharyngeal carcinoma. Cancer Lett 337:1-7, 2013

58. Dawson CW, Port RJ, Young LS: The role of the EBV-encoded latent membrane proteins LMP1 and LMP2 in the pathogenesis of nasopharyngeal carcinoma (NPC). Semin Cancer Biol 22:144-53, 2012

59. Wang D, Liebowitz D, Kieff E: An EBV membrane protein expressed in immortalized lymphocytes transforms established rodent cells. Cell 43:831-40, 1985

60. Dawson CW, Rickinson AB, Young LS: Epstein-Barr virus latent membrane protein inhibits human epithelial cell differentiation. Nature 344:777-80, 1990

61. Hanahan D, Weinberg RA: Hallmarks of cancer: the next generation. Cell 144:646-74, 2011

62. Miller WE, Earp HS, Raab-Traub N: The Epstein-Barr virus latent membrane protein 1 induces expression of the epidermal growth factor receptor. J Virol 69:4390-8, 1995

63. Kung CP, Raab-Traub N: Epstein-Barr virus latent membrane protein 1 induces expression of the epidermal growth factor receptor through effects on Bcl-3 and STAT3. J Virol 82:5486-93, 2008

64. Horikawa T, Sheen TS, Takeshita H, et al: Induction of c-Met proto-oncogene by Epstein-Barr virus latent membrane protein-1 and the correlation with cervical lymph node metastasis of nasopharyngeal carcinoma. Am J Pathol 159:27-33, 2001

65. Ren Q, Sato H, Murono S, et al: Epstein-Barr virus (EBV) latent membrane protein 1 induces interleukin-8 through the nuclear factor-kappa B signaling pathway in EBVinfected nasopharyngeal carcinoma cell line. Laryngoscope 114:855-9, 2004

66. Mainou BA, Everly DN, Jr., Raab-Traub N: Epstein-Barr virus latent membrane protein 1 CTAR1 mediates rodent and human fibroblast transformation through activation of PI3K. Oncogene 24:6917-24, 2005

67. Dawson CW, Laverick L, Morris MA, et al: Epstein-Barr virus-encoded LMP1 regulates epithelial cell motility and invasion via the ERK-MAPK pathway. J Virol $82: 3654-64,2008$

68. Tsai CN, Tsai CL, Tse KP, et al: The Epstein-Barr virus oncogene product, latent membrane protein 1, induces the downregulation of E-cadherin gene expression via activation of DNA methyltransferases. Proc Natl Acad Sci U S A 99:10084-9, 2002 
69. Horikawa T, Yang J, Kondo S, et al: Twist and epithelial-mesenchymal transition are induced by the EBV oncoprotein latent membrane protein 1 and are associated with metastatic nasopharyngeal carcinoma. Cancer Res 67:1970-8, 2007

70. Yoshizaki T, Sato H, Furukawa M, et al: The expression of matrix metalloproteinase 9 is enhanced by Epstein-Barr virus latent membrane protein 1. Proc Natl Acad Sci U S A 95:3621-6, 1998

71. Lee DC, Chua DT, Wei WI, et al: Induction of matrix metalloproteinases by EpsteinBarr virus latent membrane protein 1 isolated from nasopharyngeal carcinoma. Biomed Pharmacother 61:520-6, 2007

72. Murono S, Yoshizaki T, Sato H, et al: Aspirin inhibits tumor cell invasiveness induced by Epstein-Barr virus latent membrane protein 1 through suppression of matrix metalloproteinase-9 expression. Cancer Res 60:2555-61, 2000

73. Kondo S, Wakisaka N, Schell MJ, et al: Epstein-Barr virus latent membrane protein 1 induces the matrix metalloproteinase-1 promoter via an Ets binding site formed by a single nucleotide polymorphism: enhanced susceptibility to nasopharyngeal carcinoma. Int $\mathrm{J}$ Cancer 115:368-76, 2005

74. Liu LT, Peng JP, Chang HC, et al: RECK is a target of Epstein-Barr virus latent membrane protein 1. Oncogene 22:8263-70, 2003

75. Murono S, Inoue H, Tanabe T, et al: Induction of cyclooxygenase-2 by Epstein-Barr virus latent membrane protein 1 is involved in vascular endothelial growth factor production in nasopharyngeal carcinoma cells. Proc Natl Acad Sci U S A 98:6905-10, 2001

76. Kondo S, Seo SY, Yoshizaki T, et al: EBV latent membrane protein 1 up-regulates hypoxia-inducible factor 1alpha through Siah1-mediated down-regulation of prolyl hydroxylases 1 and 3 in nasopharyngeal epithelial cells. Cancer Res 66:9870-7, 2006

77. Wakisaka N, Kondo S, Yoshizaki T, et al: Epstein-Barr virus latent membrane protein 1 induces synthesis of hypoxia-inducible factor 1 alpha. Mol Cell Biol 24:5223-34, 2004

78. Tsuji A, Wakisaka N, Kondo S, et al: Induction of receptor for advanced glycation end products by EBV latent membrane protein 1 and its correlation with angiogenesis and cervical lymph node metastasis in nasopharyngeal carcinoma. Clin Cancer Res 14:5368-75, 2008

79. Zhao Y, Wang Y, Zeng S, et al: LMP1 expression is positively associated with metastasis of nasopharyngeal carcinoma: evidence from a meta-analysis. J Clin Pathol $65: 41-5,2012$

80. Ayadi W, Karray-Hakim H, Khabir A, et al: Aberrant methylation of p16, DLEC1, BLU and E-cadherin gene promoters in nasopharyngeal carcinoma biopsies from Tunisian patients. Anticancer Res 28:2161-7, 2008 
81. Brooks L, Yao QY, Rickinson AB, et al: Epstein-Barr virus latent gene transcription in nasopharyngeal carcinoma cells: coexpression of EBNA1, LMP1, and LMP2 transcripts. J Virol 66:2689-97, 1992

82. Kong QL, Hu LJ, Cao JY, et al: Epstein-Barr virus-encoded LMP2A induces an epithelial-mesenchymal transition and increases the number of side population stem-like cancer cells in nasopharyngeal carcinoma. PLoS Pathog 6:e1000940, 2010

83. Morrison JA, Raab-Traub N: Roles of the ITAM and PY motifs of Epstein-Barr virus latent membrane protein $2 \mathrm{~A}$ in the inhibition of epithelial cell differentiation and activation of $\{$ beta $\}$-catenin signaling. J Virol 79:2375-82, 2005

84. Scholle F, Bendt KM, Raab-Traub N: Epstein-Barr virus LMP2A transforms epithelial cells, inhibits cell differentiation, and activates Akt. J Virol 74:10681-9, 2000

85. Pegtel DM, Subramanian A, Sheen TS, et al: Epstein-Barr-virus-encoded LMP2A induces primary epithelial cell migration and invasion: possible role in nasopharyngeal carcinoma metastasis. J Virol 79:15430-42, 2005

86. Allen MD, Young LS, Dawson CW: The Epstein-Barr virus-encoded LMP2A and LMP2B proteins promote epithelial cell spreading and motility. J Virol 79:1789-802, 2005

87. Fukuda M, Longnecker R: Epstein-Barr virus latent membrane protein 2A mediates transformation through constitutive activation of the Ras/PI3-K/Akt Pathway. J Virol 81:9299-306, 2007

88. Fotheringham JA, Coalson NE, Raab-Traub N: Epstein-Barr virus latent membrane protein-2A induces ITAM/Syk- and Akt-dependent epithelial migration through alphavintegrin membrane translocation. J Virol 86:10308-20, 2012

89. Lan YY, Hsiao JR, Chang KC, et al: Epstein-Barr virus latent membrane protein $2 \mathrm{~A}$ promotes invasion of nasopharyngeal carcinoma cells through ERK/Fra-1-mediated induction of matrix metalloproteinase 9. J Virol 86:6656-67, 2012

90. Anderson LJ, Longnecker R: An auto-regulatory loop for EBV LMP2A involves activation of Notch. Virology 371:257-66, 2008

91. Longnecker R: Epstein-Barr virus latency: LMP2, a regulator or means for Epstein-Barr virus persistence? Adv Cancer Res 79:175-200, 2000

92. Shah KM, Stewart SE, Wei W, et al: The EBV-encoded latent membrane proteins, LMP2A and LMP2B, limit the actions of interferon by targeting interferon receptors for degradation. Oncogene 28:3903-14, 2009

93. Sivachandran N, Thawe NN, Frappier L: Epstein-Barr virus nuclear antigen 1 replication and segregation functions in nasopharyngeal carcinoma cell lines. J Virol $85: 10425-30,2011$ 
94. Yates JL, Warren N, Sugden B: Stable replication of plasmids derived from EpsteinBarr virus in various mammalian cells. Nature 313:812-5, 1985

95. Sugden B, Warren N: A promoter of Epstein-Barr virus that can function during latent infection can be transactivated by EBNA-1, a viral protein required for viral DNA replication during latent infection. J Virol 63:2644-9, 1989

96. Gahn TA, Sugden B: An EBNA-1-dependent enhancer acts from a distance of 10 kilobase pairs to increase expression of the Epstein-Barr virus LMP gene. J Virol 69:26336,1995

97. Yin Q, Flemington EK: siRNAs against the Epstein Barr virus latency replication factor, EBNA1, inhibit its function and growth of EBV-dependent tumor cells. Virology 346:38593, 2006

98. Sheu LF, Chen A, Meng CL, et al: Enhanced malignant progression of nasopharyngeal carcinoma cells mediated by the expression of Epstein-Barr nuclear antigen 1 in vivo. J Pathol 180:243-8, 1996

99. Gruhne B, Sompallae R, Marescotti D, et al: The Epstein-Barr virus nuclear antigen-1 promotes genomic instability via induction of reactive oxygen species. Proc Natl Acad Sci U S A 106:2313-8, 2009

100. Cao JY, Mansouri S, Frappier L: Changes in the nasopharyngeal carcinoma nuclear proteome induced by the EBNA1 protein of Epstein-Barr virus reveal potential roles for EBNA1 in metastasis and oxidative stress responses. J Virol 86:382-94, 2012

101. O'Neil JD, Owen TJ, Wood VH, et al: Epstein-Barr virus-encoded EBNA1 modulates the AP-1 transcription factor pathway in nasopharyngeal carcinoma cells and enhances angiogenesis in vitro. J Gen Virol 89:2833-42, 2008

102. Wood VH, O'Neil JD, Wei W, et al: Epstein-Barr virus-encoded EBNA1 regulates cellular gene transcription and modulates the STAT1 and TGFbeta signaling pathways. Oncogene 26:4135-47, 2007

103. Flavell JR, Baumforth KR, Wood VH, et al: Down-regulation of the TGF-beta target gene, PTPRK, by the Epstein-Barr virus encoded EBNA1 contributes to the growth and survival of Hodgkin lymphoma cells. Blood 111:292-301, 2008

104. Valentine R, Dawson CW, Hu C, et al: Epstein-Barr virus-encoded EBNA1 inhibits the canonical NF-kappaB pathway in carcinoma cells by inhibiting IKK phosphorylation. Mol Cancer 9:1, 2010

105. Holowaty MN, Sheng Y, Nguyen T, et al: Protein interaction domains of the ubiquitinspecific protease, USP7/HAUSP. J Biol Chem 278:47753-61, 2003 
106. Saridakis V, Sheng Y, Sarkari F, et al: Structure of the p53 binding domain of HAUSP/USP7 bound to Epstein-Barr nuclear antigen 1 implications for EBV-mediated immortalization. Mol Cell 18:25-36, 2005

107. Sivachandran N, Sarkari F, Frappier L: Epstein-Barr nuclear antigen 1 contributes to nasopharyngeal carcinoma through disruption of PML nuclear bodies. PLoS Pathog 4:e1000170, 2008

108. Holowaty MN, Zeghouf M, Wu H, et al: Protein profiling with Epstein-Barr nuclear antigen-1 reveals an interaction with the herpesvirus-associated ubiquitin-specific protease HAUSP/USP7. J Biol Chem 278:29987-94, 2003

109. Sivachandran N, Dawson CW, Young LS, et al: Contributions of the Epstein-Barr virus EBNA1 protein to gastric carcinoma. J Virol 86:60-8, 2012

110. Takahashi Y, Lallemand-Breitenbach V, Zhu J, et al: PML nuclear bodies and apoptosis. Oncogene 23:2819-24, 2004

111. Salomoni P, Ferguson BJ, Wyllie AH, et al: New insights into the role of PML in tumour suppression. Cell Res 18:622-40, 2008

112. Gottifredi V, Prives C: P53 and PML: new partners in tumor suppression. Trends Cell Biol 11:184-7, 2001

113. Dresang LR, Vereide DT, Sugden B: Identifying sites bound by Epstein-Barr virus nuclear antigen 1 (EBNA1) in the human genome: defining a position-weighted matrix to predict sites bound by EBNA1 in viral genomes. J Virol 83:2930-40, 2009

114. Lu F, Wikramasinghe P, Norseen J, et al: Genome-wide analysis of host-chromosome binding sites for Epstein-Barr Virus Nuclear Antigen 1 (EBNA1). Virol J 7:262, 2010

115. Canaan A, Haviv I, Urban AE, et al: EBNA1 regulates cellular gene expression by binding cellular promoters. Proc Natl Acad Sci U S A 106:22421-6, 2009

116. Takada K: Role of EBER and BARF1 in nasopharyngeal carcinoma (NPC) tumorigenesis. Semin Cancer Biol 22:162-5, 2012

117. Takada K, Nanbo A: The role of EBERs in oncogenesis. Semin Cancer Biol 11:461-7, 2001

118. Wu TC, Mann RB, Epstein JI, et al: Abundant expression of EBER1 small nuclear RNA in nasopharyngeal carcinoma. A morphologically distinctive target for detection of Epstein-Barr virus in formalin-fixed paraffin-embedded carcinoma specimens. Am J Pathol 138:1461-9, 1991

119. Tsang CM, Zhang G, Seto E, et al: Epstein-Barr virus infection in immortalized nasopharyngeal epithelial cells: regulation of infection and phenotypic characterization. Int J Cancer 127:1570-83, 2010 
120. Iwakiri D, Takada K: Role of EBERs in the pathogenesis of EBV infection. Adv Cancer Res 107:119-36, 2010

121. Iwakiri D, Zhou L, Samanta M, et al: Epstein-Barr virus (EBV)-encoded small RNA is released from EBV-infected cells and activates signaling from Toll-like receptor 3. J Exp Med 206:2091-9, 2009

122. Iwakiri D, Sheen TS, Chen JY, et al: Epstein-Barr virus-encoded small RNA induces insulin-like growth factor 1 and supports growth of nasopharyngeal carcinoma-derived cell lines. Oncogene 24:1767-73, 2005

123. Han BL, Xu XY, Zhang CZ, et al: Systematic review on Epstein-Barr virus (EBV) DNA in diagnosis of nasopharyngeal carcinoma in Asian populations. Asian Pac J Cancer Prev 13:2577-81, 2012

124. de-The G, Lavoue MF, Muenz L: Differences in EBV antibody titres of patients with nasopharyngeal carcinoma originating from high, intermediate and low incidence areas. IARC Sci Publ:471-81, 1978

125. Lennette ET, Winberg G, Yadav M, et al: Antibodies to LMP2A/2B in EBV-carrying malignancies. Eur J Cancer 31A:1875-8, 1995

126. Xu J, Ahmad A, D'Addario M, et al: Analysis and significance of anti-latent membrane protein-1 antibodies in the sera of patients with EBV-associated diseases. $\mathrm{J}$ Immunol 164:2815-22, 2000

127. Lo YM, Chan LY, Lo KW, et al: Quantitative analysis of cell-free Epstein-Barr virus DNA in plasma of patients with nasopharyngeal carcinoma. Cancer Res 59:1188-91, 1999

128. Lin JC, Wang WY, Chen KY, et al: Quantification of plasma Epstein-Barr virus DNA in patients with advanced nasopharyngeal carcinoma. N Engl J Med 350:2461-70, 2004

129. Yu KJ, Hsu WL, Pfeiffer RM, et al: Prognostic utility of anti-EBV antibody testing for defining NPC risk among individuals from high-risk NPC families. Clin Cancer Res 17:1906-14, 2011

130. Zeng Y, Zhang LG, Li HY, et al: Serological mass survey for early detection of nasopharyngeal carcinoma in Wuzhou City, China. Int J Cancer 29:139-41, 1982

131. Zeng Y, Zhong JM, Li LY, et al: Follow-up studies on Epstein-Barr virus IgA/VCA antibody-positive persons in Zangwu County, China. Intervirology 20:190-4, 1983

132. Zeng Y, Zhang LG, Wu YC, et al: Prospective studies on nasopharyngeal carcinoma in Epstein-Barr virus IgA/VCA antibody-positive persons in Wuzhou City, China. Int J Cancer 36:545-7, 1985 
133. Baizig NM, Morand P, Seigneurin JM, et al: Complementary determination of EpsteinBarr virus DNA load and serum markers for nasopharyngeal carcinoma screening and early detection in individuals at risk in Tunisia. Eur Arch Otorhinolaryngol 269:1005-11, 2012

134. de-Vathaire F, Sancho-Garnier H, de-The H, et al: Prognostic value of EBV markers in the clinical management of nasopharyngeal carcinoma (NPC): a multicenter follow-up study. Int J Cancer 42:176-81, 1988

135. Twu CW, Wang WY, Liang WM, et al: Comparison of the prognostic impact of serum anti-EBV antibody and plasma EBV DNA assays in nasopharyngeal carcinoma. Int J Radiat Oncol Biol Phys 67:130-7, 2007

136. Wang WY, Twu CW, Lin WY, et al: Plasma Epstein-Barr virus DNA screening followed by (1)(8)F-fluoro-2-deoxy-D-glucose positron emission tomography in detecting posttreatment failures of nasopharyngeal carcinoma. Cancer 117:4452-9, 2011

137. Chan JY, Wong ST: The role of plasma Epstein-Barr virus DNA in the management of recurrent nasopharyngeal carcinoma. Laryngoscope, 2013

138. Hsu CL, Chan SC, Chang KP, et al: Clinical scenario of EBV DNA follow-up in patients of treated localized nasopharyngeal carcinoma. Oral Oncol 49:620-5, 2013

139. Hsu CL, Chang KP, Lin CY, et al: Plasma Epstein-Barr virus DNA concentration and clearance rate as novel prognostic factors for metastatic nasopharyngeal carcinoma. Head Neck 34:1064-70, 2012

140. Hou X, Zhao C, Guo Y, et al: Different clinical significance of pre- and post-treatment plasma Epstein-Barr virus DNA load in nasopharyngeal carcinoma treated with radiotherapy. Clin Oncol (R Coll Radiol) 23:128-33, 2011

141. Lin JC, Chen KY, Wang WY, et al: Detection of Epstein-Barr virus DNA in the peripheral-blood cells of patients with nasopharyngeal carcinoma: relationship to distant metastasis and survival. J Clin Oncol 19:2607-15, 2001

142. Le QT, Zhang Q, Cao H, et al: An international collaboration to harmonize the quantitative plasma Epstein-Barr virus DNA assay for future biomarker-guided trials in nasopharyngeal carcinoma. Clin Cancer Res 19:2208-15, 2013

143. Houali K, Wang X, Shimizu Y, et al: A new diagnostic marker for secreted EpsteinBarr virus encoded LMP1 and BARF1 oncoproteins in the serum and saliva of patients with nasopharyngeal carcinoma. Clin Cancer Res 13:4993-5000, 2007

144. Lo KW, Lo YM, Leung SF, et al: Analysis of cell-free Epstein-Barr virus associated RNA in the plasma of patients with nasopharyngeal carcinoma. Clin Chem 45:1292-4, 1999

145. Gourzones C, Gelin A, Bombik I, et al: Extra-cellular release and blood diffusion of BART viral micro-RNAs produced by EBV-infected nasopharyngeal carcinoma cells. Virol J 7:271, 2010 
146. Wong AM, Kong KL, Tsang JW, et al: Profiling of Epstein-Barr virus-encoded microRNAs in nasopharyngeal carcinoma reveals potential biomarkers and oncomirs. Cancer 118:698-710, 2012

147. Chan JY, Gao W, Ho WK, et al: Overexpression of Epstein-Barr virus-encoded microRNA-BART7 in undifferentiated nasopharyngeal carcinoma. Anticancer Res 32:3201-10, 2012

148. Busson P: Nasopharyngeal carcinoma : keys for translational medicine and biology. New York, N.Y. Landes Bioscience, 2013

149. Wong TS, Kwong DL, Sham JS, et al: Quantitative plasma hypermethylated DNA markers of undifferentiated nasopharyngeal carcinoma. Clin Cancer Res 10:2401-6, 2004

150. Tian F, Yip SP, Kwong DL, et al: Promoter hypermethylation of tumor suppressor genes in serum as potential biomarker for the diagnosis of nasopharyngeal carcinoma. Cancer Epidemiol 37:708-13, 2013

151. Chen C, Chen S, Le QT, et al: A prognostic model for distant metastasis in locally advanced nasopharyngeal carcinoma after concurrent chemoradiotherapy. Head Neck:[epub ahead of print], 2013

152. Xu J, Menezes J, Prasad U, et al: Elevated serum levels of transforming growth factor betal in Epstein-Barr virus-associated nasopharyngeal carcinoma patients. Int J Cancer 84:396-9, 1999

153. Li J, Zeng XH, Mo HY, et al: Functional inactivation of EBV-specific T-lymphocytes in nasopharyngeal carcinoma: implications for tumor immunotherapy. PLoS One 2:e1122, 2007

154. Caggiari L, Guidoboni M, Vaccher E, et al: High serum levels of soluble CD40-L in patients with undifferentiated nasopharyngeal carcinoma: pathogenic and clinical relevance. Infect Agent Cancer 2:5, 2007

155. Chang KP, Hao SP, Chang JH, et al: Macrophage inflammatory protein-3alpha is a novel serum marker for nasopharyngeal carcinoma detection and prediction of treatment outcomes. Clin Cancer Res 14:6979-87, 2008

156. Rousselet G, Bachouchi M, Busson P, et al: Clinical implications of the serum level of CD23 in patients with undifferentiated nasopharyngeal carcinoma. J Clin Oncol 11:2143-9, 1993

157. Yip KW, Shi W, Pintilie M, et al: Prognostic significance of the Epstein-Barr virus, p53, Bcl-2, and survivin in nasopharyngeal cancer. Clin Cancer Res 12:5726-32, 2006

158. Qian CN, Guo X, Cao B, et al: Met protein expression level correlates with survival in patients with late-stage nasopharyngeal carcinoma. Cancer Res 62:589-96, 2002 
159. Bartel DP: MicroRNAs: genomics, biogenesis, mechanism, and function. Cell 116:281-97, 2004

160. Esquela-Kerscher A, Slack FJ: Oncomirs - microRNAs with a role in cancer. Nat Rev Cancer 6:259-69, 2006

161. Breving K, Esquela-Kerscher A: The complexities of microRNA regulation: mirandering around the rules. Int J Biochem Cell Biol 42:1316-29, 2009

162. Borchert GM, Lanier W, Davidson BL: RNA polymerase III transcribes human microRNAs. Nat Struct Mol Biol 13:1097-101, 2006

163. Lee YS, Dutta A: MicroRNAs in cancer. Annu Rev Pathol 4:199-227, 2009

164. Kim VN, Han J, Siomi MC: Biogenesis of small RNAs in animals. Nat Rev Mol Cell Biol 10:126-39, 2009

165. Schwarz DS, Hutvagner G, Du T, et al: Asymmetry in the assembly of the RNAi enzyme complex. Cell 115:199-208, 2003

166. Jin P, Zarnescu DC, Ceman S, et al: Biochemical and genetic interaction between the fragile X mental retardation protein and the microRNA pathway. Nat Neurosci 7:113-7, 2004

167. Chu CY, Rana TM: Translation repression in human cells by microRNA-induced gene silencing requires RCK/p54. PLoS Biol 4:e210, 2006

168. Eulalio A, Rehwinkel J, Stricker M, et al: Target-specific requirements for enhancers of decapping in miRNA-mediated gene silencing. Genes Dev 21:2558-70, 2007

169. Krek A, Grun D, Poy MN, et al: Combinatorial microRNA target predictions. Nat Genet 37:495-500, 2005

170. Lewis BP, Burge CB, Bartel DP: Conserved seed pairing, often flanked by adenosines, indicates that thousands of human genes are microRNA targets. Cell 120:15-20, 2005

171. John B, Enright AJ, Aravin A, et al: Human MicroRNA targets. PLoS Biol 2:e363, 2004

172. Liu J, Valencia-Sanchez MA, Hannon GJ, et al: MicroRNA-dependent localization of targeted mRNAs to mammalian P-bodies. Nat Cell Biol 7:719-23, 2005

173. Orom UA, Nielsen FC, Lund AH: MicroRNA-10a binds the 5'UTR of ribosomal protein mRNAs and enhances their translation. Mol Cell 30:460-71, 2008

174. Subramanian S, Lui WO, Lee $\mathrm{CH}$, et al: MicroRNA expression signature of human sarcomas. Oncogene 27:2015-26, 2008 
175. Osaki M, Takeshita F, Ochiya T: MicroRNAs as biomarkers and therapeutic drugs in human cancer. Biomarkers 13:658-70, 2008

176. Jay C, Nemunaitis J, Chen P, et al: miRNA profiling for diagnosis and prognosis of human cancer. DNA Cell Biol 26:293-300, 2007

177. Nair VS, Maeda LS, Ioannidis JP: Clinical outcome prediction by microRNAs in human cancer: a systematic review. J Natl Cancer Inst 104:528-40, 2012

178. Yanaihara N, Caplen N, Bowman E, et al: Unique microRNA molecular profiles in lung cancer diagnosis and prognosis. Cancer Cell 9:189-98, 2006

179. Yu SL, Chen HY, Chang GC, et al: MicroRNA signature predicts survival and relapse in lung cancer. Cancer Cell 13:48-57, 2008

180. Iorio MV, Ferracin M, Liu CG, et al: MicroRNA gene expression deregulation in human breast cancer. Cancer Res 65:7065-70, 2005

181. Ciafre SA, Galardi S, Mangiola A, et al: Extensive modulation of a set of microRNAs in primary glioblastoma. Biochem Biophys Res Commun 334:1351-8, 2005

182. Calin GA, Ferracin M, Cimmino A, et al: A MicroRNA signature associated with prognosis and progression in chronic lymphocytic leukemia. N Engl J Med 353:1793-801, 2005

183. Liu N, Chen NY, Cui RX, et al: Prognostic value of a microRNA signature in nasopharyngeal carcinoma: a microRNA expression analysis. Lancet Oncol 13:633-41, 2012

184. Sengupta S, den Boon JA, Chen IH, et al: MicroRNA 29c is down-regulated in nasopharyngeal carcinomas, up-regulating mRNAs encoding extracellular matrix proteins. Proc Natl Acad Sci U S A 105:5874-8, 2008

185. Liu X, Luo HN, Tian WD, et al: Diagnostic and prognostic value of plasma microRNA deregulation in nasopharyngeal carcinoma. Cancer Biol Ther 14, 2013

186. Li T, Chen JX, Fu XP, et al: microRNA expression profiling of nasopharyngeal carcinoma. Oncol Rep 25:1353-63, 2011

187. Luo Z, Dai Y, Zhang L, et al: miR-18a promotes malignant progression by impairing microRNA biogenesis in nasopharyngeal carcinoma. Carcinogenesis 34:415-25, 2013

188. Lu J, Xu X, Liu X, et al: Predictive value of miR-9 as a potential biomarker for nasopharyngeal carcinoma metastasis. Br J Cancer, 2013

189. Zeng X, Xiang J, Wu M, et al: Circulating miR-17, miR-20a, miR-29c, and miR-223 combined as non-invasive biomarkers in nasopharyngeal carcinoma. PLoS One 7:e46367, 2012 
190. Liu N, Cui RX, Sun Y, et al: A four-miRNA signature identified from genome-wide serum miRNA profiling predicts survival in patients with nasopharyngeal carcinoma. Int $\mathbf{J}$ Cancer, 2013

191. Alajez NM, Shi W, Hui AB, et al: Enhancer of Zeste homolog 2 (EZH2) is overexpressed in recurrent nasopharyngeal carcinoma and is regulated by miR-26a, miR101, and miR-98. Cell Death Dis 1:e85, 2010

192. Zhang JX, Qian D, Wang FW, et al: MicroRNA-29c enhances the sensitivities of human nasopharyngeal carcinoma to cisplatin-based chemotherapy and radiotherapy. Cancer Lett 329:91-8, 2013

193. Liu N, Jiang N, Guo R, et al: MiR-451 inhibits cell growth and invasion by targeting MIF and is associated with survival in nasopharyngeal carcinoma. Mol Cancer 12:123, 2013

194. Zhang L, Deng T, Li X, et al: microRNA-141 is involved in a nasopharyngeal carcinoma-related genes network. Carcinogenesis 31:559-66, 2010

195. Zhang LY, Ho-Fun Lee V, Wong AM, et al: MicroRNA-144 promotes cell proliferation, migration and invasion in nasopharyngeal carcinoma through repression of PTEN. Carcinogenesis 34:454-63, 2013

196. Lung RW, Wang X, Tong JH, et al: A single nucleotide polymorphism in microRNA146a is associated with the risk for nasopharyngeal carcinoma. Mol Carcinog 52 Suppl 1:E28-38, 2013

197. Zhao Y, Chen X, Jing M, et al: Expression of miRNA-146a in nasopharyngeal carcinoma is upregulated by Epstein-Barr virus latent membrane protein 1. Oncol Rep 28:1237-42, 2012

198. Liu X, Lv XB, Wang XP, et al: MiR-138 suppressed nasopharyngeal carcinoma growth and tumorigenesis by targeting the CCND1 oncogene. Cell Cycle 11:2495-506, 2012

199. Du ZM, Hu LF, Wang HY, et al: Upregulation of MiR-155 in nasopharyngeal carcinoma is partly driven by LMP1 and LMP2A and downregulates a negative prognostic marker JMJD1A. PLoS One 6:e19137, 2011

200. Zhu X, Wang Y, Sun Y, et al: MiR-155 up-regulation by LMP1 DNA contributes to increased nasopharyngeal carcinoma cell proliferation and migration. Eur Arch Otorhinolaryngol, 2013

201. Yang X, Ni W, Lei K: miR-200b Suppresses Cell Growth, Migration and Invasion by Targeting Notch1 in Nasopharyngeal Carcinoma. Cell Physiol Biochem 32:1288-98, 2013

202. Deng M, Ye Q, Qin Z, et al: miR-214 promotes tumorigenesis by targeting lactotransferrin in nasopharyngeal carcinoma. Tumour Biol 34:1793-800, 2013 
203. Deng M, Tang H, Zhou Y, et al: miR-216b suppresses tumor growth and invasion by targeting KRAS in nasopharyngeal carcinoma. J Cell Sci 124:2997-3005, 2011

204. Alajez NM, Lenarduzzi M, Ito E, et al: MiR-218 suppresses nasopharyngeal cancer progression through downregulation of survivin and the SLIT2-ROBO1 pathway. Cancer Res 71:2381-91, 2011

205. Yi C, Wang Q, Wang L, et al: MiR-663, a microRNA targeting p21(WAF1/CIP1), promotes the proliferation and tumorigenesis of nasopharyngeal carcinoma. Oncogene $31: 4421-33,2012$

206. Liu N, Tang LL, Sun Y, et al: MiR-29c suppresses invasion and metastasis by targeting TIAM1 in nasopharyngeal carcinoma. Cancer Lett 329:181-8, 2013

207. Lu J, Luo H, Liu X, et al: miR-9 targets CXCR4 and functions as a potential tumor suppressor in nasopharyngeal carcinoma. Carcinogenesis, 2013

208. Gao F, Zhao ZL, Zhao WT, et al: miR-9 modulates the expression of interferonregulated genes and MHC class I molecules in human nasopharyngeal carcinoma cells. Biochem Biophys Res Commun 431:610-6, 2013

209. Lu J, He ML, Wang L, et al: MiR-26a inhibits cell growth and tumorigenesis of nasopharyngeal carcinoma through repression of EZH2. Cancer Res 71:225-33, 2011

210. Yu L, Lu J, Zhang B, et al: miR-26a inhibits invasion and metastasis of nasopharyngeal cancer by targeting EZH2. Oncol Lett 5:1223-1228, 2013

211. Zhu X, Wang Y, Sun Y, et al: MiR-155 up-regulation by LMP1 DNA contributes to increased nasopharyngeal carcinoma cell proliferation and migration. Eur Arch Otorhinolaryngol:[epub ahead of print], 2013

212. Xia H, Cheung WK, Sze J, et al: miR-200a regulates epithelial-mesenchymal to stemlike transition via ZEB2 and beta-catenin signaling. J Biol Chem 285:36995-7004, 2010

213. Xia H, Ng SS, Jiang S, et al: miR-200a-mediated downregulation of ZEB2 and CTNNB1 differentially inhibits nasopharyngeal carcinoma cell growth, migration and invasion. Biochem Biophys Res Commun 391:535-41, 2010

214. Qu C, Liang Z, Huang J, et al: MiR-205 determines the radioresistance of human nasopharyngeal carcinoma by directly targeting PTEN. Cell Cycle 11:785-96, 2012

215. Wang D, Wang S, Liu Q, et al: SZ-685C exhibits potent anticancer activity in both radiosensitive and radioresistant NPC cells through the miR-205-PTEN-Akt pathway. Oncol Rep 29:2341-7, 2013

216. Pfeffer S, Zavolan M, Grasser FA, et al: Identification of virus-encoded microRNAs. Science 304:734-6, 2004 
217. Cosmopoulos K, Pegtel M, Hawkins J, et al: Comprehensive profiling of Epstein-Barr virus microRNAs in nasopharyngeal carcinoma. J Virol 83:2357-67, 2009

218. Zhu JY, Pfuhl T, Motsch N, et al: Identification of novel Epstein-Barr virus microRNA genes from nasopharyngeal carcinomas. J Virol 83:3333-41, 2009

219. Lo AK, To KF, Lo KW, et al: Modulation of LMP1 protein expression by EBVencoded microRNAs. Proc Natl Acad Sci U S A 104:16164-9, 2007

220. Ramakrishnan R, Donahue H, Garcia D, et al: Epstein-Barr virus BART9 miRNA modulates LMP1 levels and affects growth rate of nasal NK T cell lymphomas. PLoS One 6:e27271, 2011

221. Lung RW, Tong JH, Sung YM, et al: Modulation of LMP2A expression by a newly identified Epstein-Barr virus-encoded microRNA miR-BART22. Neoplasia 11:1174-84, 2009

222. Choy EY, Siu KL, Kok KH, et al: An Epstein-Barr virus-encoded microRNA targets PUMA to promote host cell survival. J Exp Med 205:2551-60, 2008

223. Marquitz AR, Mathur A, Nam CS, et al: The Epstein-Barr Virus BART microRNAs target the pro-apoptotic protein Bim. Virology 412:392-400, 2011

224. Dolken L, Malterer G, Erhard F, et al: Systematic analysis of viral and cellular microRNA targets in cells latently infected with human gamma-herpesviruses by RISC immunoprecipitation assay. Cell Host Microbe 7:324-34, 2010

225. Nachmani D, Stern-Ginossar N, Sarid R, et al: Diverse herpesvirus microRNAs target the stress-induced immune ligand MICB to escape recognition by natural killer cells. Cell Host Microbe 5:376-85, 2009

226. Yang IV, Wade CM, Kang HM, et al: Identification of novel genes that mediate innate immunity using inbred mice. Genetics 183:1535-44, 2009

227. Iizasa H, Wulff BE, Alla NR, et al: Editing of Epstein-Barr virus-encoded BART6 microRNAs controls their dicer targeting and consequently affects viral latency. J Biol Chem 285:33358-70, 2010

228. Shi R, Sun YH, Zhang XH, et al: Poly(T) adaptor RT-PCR. Methods Mol Biol 822:5366,2012

229. Chen C, Ridzon DA, Broomer AJ, et al: Real-time quantification of microRNAs by stem-loop RT-PCR. Nucleic Acids Res 33:e179, 2005

230. Lennox KA, Behlke MA: Chemical modification and design of anti-miRNA

oligonucleotides. Gene Ther 18:1111-20, 2011 
231. Petersen M, Wengel J: LNA: a versatile tool for therapeutics and genomics. Trends Biotechnol 21:74-81, 2003

232. Heller MJ: DNA microarray technology: devices, systems, and applications. Annu Rev Biomed Eng 4:129-53, 2002

233. Fan J-B: Next-generation MicroRNA expression profiling technology : methods and protocols. New York, Humana Press, 2012

234. Kolbert CP, Feddersen RM, Rakhshan F, et al: Multi-platform analysis of microRNA expression measurements in RNA from fresh frozen and FFPE tissues. PLoS One 8:e52517, 2013

235. Meyer SU, Pfaffl MW, Ulbrich SE: Normalization strategies for microRNA profiling experiments: a 'normal' way to a hidden layer of complexity? Biotechnol Lett 32:1777-88, 2010

236. Pritchard CC, Cheng HH, Tewari M: MicroRNA profiling: approaches and considerations. Nat Rev Genet 13:358-69, 2012

237. Knutsen E, Fiskaa T, Ursvik A, et al: Performance comparison of digital microRNA profiling technologies applied on human breast cancer cell lines. PLoS One 8:e75813, 2013

238. Pradervand S, Weber J, Lemoine F, et al: Concordance among digital gene expression, microarrays, and qPCR when measuring differential expression of microRNAs.

Biotechniques 48:219-22, 2010

239. Callari M, Dugo M, Musella V, et al: Comparison of microarray platforms for measuring differential microRNA expression in paired normal/cancer colon tissues. PLoS One $7: e 45105,2012$

240. Sato F, Tsuchiya S, Terasawa K, et al: Intra-platform repeatability and inter-platform comparability of microRNA microarray technology. PLoS One 4:e5540, 2009

241. Git A, Dvinge H, Salmon-Divon M, et al: Systematic comparison of microarray profiling, real-time PCR, and next-generation sequencing technologies for measuring differential microRNA expression. RNA 16:991-1006, 2010

242. Yauk CL, Rowan-Carroll A, Stead JD, et al: Cross-platform analysis of global microRNA expression technologies. BMC Genomics 11:330, 2010

243. Meyer SU, Kaiser S, Wagner C, et al: Profound effect of profiling platform and normalization strategy on detection of differentially expressed microRNAs--a comparative study. PLoS One 7:e38946, 2012

244. Gee HE, Buffa FM, Camps C, et al: The small-nucleolar RNAs commonly used for microRNA normalisation correlate with tumour pathology and prognosis. Br J Cancer 104:1168-77, 2011 
245. Mourtada-Maarabouni M, Pickard MR, Hedge VL, et al: GAS5, a non-protein-coding RNA, controls apoptosis and is downregulated in breast cancer. Oncogene 28:195-208, 2009

246. Williams GT, Farzaneh F: Are snoRNAs and snoRNA host genes new players in cancer? Nat Rev Cancer 12:84-8, 2012

247. Pradervand $\mathrm{S}$, Weber J, Thomas J, et al: Impact of normalization on miRNA microarray expression profiling. RNA 15:493-501, 2009

248. Mestdagh P, Van Vlierberghe P, De Weer A, et al: A novel and universal method for microRNA RT-qPCR data normalization. Genome Biol 10:R64, 2009

249. Pan Q, Luo X, Chegini N: Differential expression of microRNAs in myometrium and leiomyomas and regulation by ovarian steroids. J Cell Mol Med 12:227-40, 2008

250. Davison TS, Johnson CD, Andruss BF: Analyzing micro-RNA expression using microarrays. Methods Enzymol 411:14-34, 2006

251. Garmire LX, Subramaniam S: Evaluation of normalization methods in mammalian microRNA-Seq data. RNA 18:1279-88, 2012

252. Lai SZ, Li WF, Chen L, et al: How does intensity-modulated radiotherapy versus conventional two-dimensional radiotherapy influence the treatment results in nasopharyngeal carcinoma patients? Int J Radiat Oncol Biol Phys 80:661-8, 2011

253. Xiao WW, Huang SM, Han F, et al: Local control, survival, and late toxicities of locally advanced nasopharyngeal carcinoma treated by simultaneous modulated accelerated radiotherapy combined with cisplatin concurrent chemotherapy: long-term results of a phase 2 study. Cancer 117:1874-83, 2011

254. Selbach M, Schwanhausser B, Thierfelder N, et al: Widespread changes in protein synthesis induced by microRNAs. Nature 455:58-63, 2008

255. Friedman RC, Farh KK, Burge CB, et al: Most mammalian mRNAs are conserved targets of microRNAs. Genome Res 19:92-105, 2009

256. Iorio MV, Croce CM: microRNA involvement in human cancer. Carcinogenesis $33: 1126-33,2012$

257. Jemal A, Murray T, Ward E, et al: Cancer statistics, 2005. CA Cancer J Clin 55:10-30, 2005

258. Forastiere AA, Trotti A, Pfister DG, et al: Head and neck cancer: recent advances and new standards of care. J Clin Oncol 24:2603-5, 2006

259. Calin GA, Croce CM: MicroRNA signatures in human cancers. Nat Rev Cancer 6:85766,2006 
260. Hui AB, Lenarduzzi M, Krushel T, et al: Comprehensive MicroRNA profiling for head and neck squamous cell carcinomas. Clin Cancer Res 16:1129-39, 2010

261. Su ZZ, Kang DC, Chen Y, et al: Identification and cloning of human astrocyte genes displaying elevated expression after infection with HIV-1 or exposure to HIV-1 envelope glycoprotein by rapid subtraction hybridization, RaSH. Oncogene 21:3592-602, 2002

262. Hu G, Wei Y, Kang Y: The multifaceted role of MTDH/AEG-1 in cancer progression. Clin Cancer Res 15:5615-20, 2009

263. Jian-Bo X, Hui W, Yu-Long H, et al: Astrocyte-elevated gene-1 overexpression is associated with poor prognosis in gastric cancer. Med Oncol 28:455-62, 2010

264. Cummings B, Keane T, Pintilie M, et al: Five year results of a randomized trial comparing hyperfractionated to conventional radiotherapy over four weeks in locally advanced head and neck cancer. Radiother Oncol 85:7-16, 2007

265. Hui AB, Shi W, Boutros PC, et al: Robust global micro-RNA profiling with formalinfixed paraffin-embedded breast cancer tissues. Lab Invest 89:597-606, 2009

266. Livak KJ, Schmittgen TD: Analysis of relative gene expression data using real-time quantitative PCR and the 2(-Delta Delta C(T)) Method. Methods 25:402-8, 2001

267. Alajez NM, Shi W, Hui AB, et al: Targeted depletion of BMI1 sensitizes tumor cells to P53-mediated apoptosis in response to radiation therapy. Cell Death Differ 16:1469-79, 2009

268. Furuta M, Kozaki KI, Tanaka S, et al: miR-124 and miR-203 are epigenetically silenced tumor-suppressive microRNAs in hepatocellular carcinoma. Carcinogenesis 31:766-76, 2010

270. Poy MN, Eliasson L, Krutzfeldt J, et al: A pancreatic islet-specific microRNA regulates insulin secretion. Nature 432:226-30, 2004

271. Tsukamoto Y, Nakada C, Noguchi T, et al: MicroRNA-375 is downregulated in gastric carcinomas and regulates cell survival by targeting PDK1 and 14-3-3zeta. Cancer Res 70:2339-49, 2010

272. Lee SG, Su ZZ, Emdad L, et al: Astrocyte elevated gene-1 activates cell survival pathways through PI3K-Akt signaling. Oncogene 27:1114-21, 2008

273. Kikuno N, Shiina H, Urakami S, et al: Knockdown of astrocyte-elevated gene-1 inhibits prostate cancer progression through upregulation of FOXO3a activity. Oncogene 26:7647-55, 2007

274. Li J, Yang L, Song L, et al: Astrocyte elevated gene-1 is a proliferation promoter in breast cancer via suppressing transcriptional factor FOXO1. Oncogene 28:3188-96, 2009 
275. Nohata N, Hanazawa T, Kikkawa N, et al: Tumor suppressive microRNA-375 regulates oncogene AEG-1/MTDH in head and neck squamous cell carcinoma (HNSCC). J Hum Genet 56:595-601, 2011

276. Kloosterman WP, Lagendijk AK, Ketting RF, et al: Targeted inhibition of miRNA maturation with morpholinos reveals a role for miR-375 in pancreatic islet development. PLoS Biol 5:e203, 2007

277. Poy MN, Hausser J, Trajkovski M, et al: miR-375 maintains normal pancreatic alphaand beta-cell mass. Proc Natl Acad Sci U S A 106:5813-8, 2009

278. Hinton A, Afrikanova I, Wilson M, et al: A distinct microRNA signature for definitive endoderm derived from human embryonic stem cells. Stem Cells Dev 19:797-807, 2010

279. Avissar M, McClean MD, Kelsey KT, et al: MicroRNA expression in head and neck cancer associates with alcohol consumption and survival. Carcinogenesis 30:2059-63, 2009

280. Ding L, Xu Y, Zhang W, et al: MiR-375 frequently downregulated in gastric cancer inhibits cell proliferation by targeting JAK2. Cell Res 20:784-93, 2010

281. Mathe EA, Nguyen GH, Bowman ED, et al: MicroRNA expression in squamous cell carcinoma and adenocarcinoma of the esophagus: associations with survival. Clin Cancer Res 15:6192-200, 2009

282. Nguyen GH, Schetter AJ, Chou DB, et al: Inflammatory and microRNA gene expression as prognostic classifier of Barrett's-associated esophageal adenocarcinoma. Clin Cancer Res 16:5824-34, 2010

283. Wach S, Nolte E, Szczyrba J, et al: MicroRNA profiles of prostate carcinoma detected by multiplatform microRNA screening. Int J Cancer 130:611-21, 2011

284. Zhang X, Yan Z, Zhang J, et al: Combination of hsa-miR-375 and hsa-miR-142-5p as a predictor for recurrence risk in gastric cancer patients following surgical resection. Ann Oncol 22:2257-66, 2011

285. Yamamoto N, Mizoe JE, Numasawa H, et al: Allelic loss of chromosome 2 in human oral squamous cell carcinoma: correlation with lymph node metastasis. Oral Oncol 39:64-8, 2003

286. Zhang B, Liu XX, He JR, et al: Pathologically decreased miR-26a antagonizes apoptosis and facilitates carcinogenesis by targeting MTDH and EZH2 in breast cancer. Carcinogenesis 32:2-9, 2011

287. Ashman JN, Patmore HS, Condon LT, et al: Prognostic value of genomic alterations in head and neck squamous cell carcinoma detected by comparative genomic hybridisation. $\mathrm{Br}$ J Cancer 89:864-9, 2003 
288. Yoo BK, Emdad L, Su ZZ, et al: Astrocyte elevated gene-1 regulates hepatocellular carcinoma development and progression. J Clin Invest 119:465-77, 2009

289. Brown DM, Ruoslahti E: Metadherin, a cell surface protein in breast tumors that mediates lung metastasis. Cancer Cell 5:365-74, 2004

290. Yu C, Chen K, Zheng H, et al: Overexpression of astrocyte elevated gene-1 (AEG-1) is associated with esophageal squamous cell carcinoma (ESCC) progression and pathogenesis. Carcinogenesis 30:894-901, 2009

291. Chen W, Ke Z, Shi H, et al: Overexpression of AEG-1 in renal cell carcinoma and its correlation with tumor nuclear grade and progression. Neoplasma 57:522-9, 2011

292. Song H, Li C, Li R, et al: Prognostic significance of AEG-1 expression in colorectal carcinoma. Int J Colorectal Dis, 2010

293. Song L, Li W, Zhang H, et al: Over-expression of AEG-1 significantly associates with tumour aggressiveness and poor prognosis in human non-small cell lung cancer. J Pathol 219:317-26, 2009

294. Sarkar D, Park ES, Emdad L, et al: Molecular basis of nuclear factor-kappaB activation by astrocyte elevated gene-1. Cancer Res 68:1478-84, 2008

295. Liu L, Wu J, Ying Z, et al: Astrocyte elevated gene-1 upregulates matrix metalloproteinase-9 and induces human glioma invasion. Cancer Res 70:3750-9, 2010

296. Lukk M, Kapushesky M, Nikkila J, et al: A global map of human gene expression. Nat Biotechnol 28:322-4, 2010

297. Qian BJ, Yan F, Li N, et al: MTDH/AEG-1-based DNA vaccine suppresses lung metastasis and enhances chemosensitivity to doxorubicin in breast cancer. Cancer Immunol Immunother 60:883-93, 2011

298. Lee AW, Ng WT, Chan YH, et al: The battle against nasopharyngeal cancer. Radiother Oncol 104:272-8, 2012

299. Sze WM, Lee AW, Yau TK, et al: Primary tumor volume of nasopharyngeal carcinoma: prognostic significance for local control. Int J Radiat Oncol Biol Phys 59:21-7, 2004

300. Leung SF, Zee B, Ma BB, et al: Plasma Epstein-Barr viral deoxyribonucleic acid quantitation complements tumor-node-metastasis staging prognostication in nasopharyngeal carcinoma. J Clin Oncol 24:5414-8, 2006

301. Wang HY, Sun BY, Zhu ZH, et al: Eight-signature classifier for prediction of nasopharyngeal carcinoma survival. J Clin Oncol 29:4516-25, 2011 
302. Chua DT, Nicholls JM, Sham JS, et al: Prognostic value of epidermal growth factor receptor expression in patients with advanced stage nasopharyngeal carcinoma treated with induction chemotherapy and radiotherapy. Int J Radiat Oncol Biol Phys 59:11-20, 2004

303. Lee RC, Feinbaum RL, Ambros V: The C. elegans heterochronic gene lin-4 encodes small RNAs with antisense complementarity to lin-14. Cell 75:843-54, 1993

304. Waggott D, Chu K, Yin S, et al: NanoStringNorm: an extensible R package for the pre-processing of NanoString mRNA and miRNA data. Bioinformatics 28:1546-8, 2012

305. Huber W, von Heydebreck A, Sultmann H, et al: Variance stabilization applied to microarray data calibration and to the quantification of differential expression. Bioinformatics 18 Suppl 1:S96-104, 2002

306. Benjamini Y, Hochberg Y: Controlling the False Discovery Rate - a Practical and Powerful Approach to Multiple Testing. Journal of the Royal Statistical Society Series BMethodological 57:289-300, 1995

307. Langsrud O: 50-50 multivariate analysis of variance for collinear responses. Journal of the Royal Statistical Society Series D-the Statistician 51:305-317, 2002

308. Tibshirani R: The lasso method for variable selection in the Cox model. Stat Med $16: 385-95,1997$

309. Goeman JJ: L1 penalized estimation in the Cox proportional hazards model. Biom J 52:70-84, 2010

310. Vittinghoff E, McCulloch CE: Relaxing the rule of ten events per variable in logistic and Cox regression. Am J Epidemiol 165:710-8, 2007

311. Heagerty PJ, Lumley T, Pepe MS: Time-dependent ROC curves for censored survival data and a diagnostic marker. Biometrics 56:337-44, 2000

312. Li JCA: Modeling survival data: Extending the Cox model. Sociological Methods \& Research 32:117-120, 2003

313. Dweep H, Sticht C, Pandey P, et al: miRWalk--database: prediction of possible miRNA binding sites by "walking" the genes of three genomes. J Biomed Inform 44:83947,2011

314. Huang da W, Sherman BT, Lempicki RA: Systematic and integrative analysis of large gene lists using DAVID bioinformatics resources. Nat Protoc 4:44-57, 2009

315. Hui AB, Bruce JP, Alajez NM, et al: Significance of dysregulated metadherin and microRNA-375 in head and neck cancer. Clin Cancer Res 17:7539-50, 2011

316. Chen HC, Chen GH, Chen YH, et al: MicroRNA deregulation and pathway alterations in nasopharyngeal carcinoma. Br J Cancer 100:1002-11, 2009 
317. Iorio MV, Croce CM: MicroRNA dysregulation in cancer: diagnostics, monitoring and therapeutics. A comprehensive review. EMBO Mol Med 4:143-59, 2012

318. Chen Y, Sun Y, Liang SB, et al: Progress report of a randomized trial comparing longterm survival and late toxicity of concurrent chemoradiotherapy with adjuvant chemotherapy versus radiotherapy alone in patients with stage III to IVB nasopharyngeal carcinoma from endemic regions of China. Cancer 119:2230-8, 2013

319. Yu HS, Wang X, Song AQ, et al: Concurrent chemoradiotherapy versus radiotherapy alone for locoregionally advanced nasopharyngeal carcinoma. Asian Pac J Cancer Prev $13: 3961-5,2012$

320. Lee CC, Ho CY: Post-treatment late complications of nasopharyngeal carcinoma. Eur Arch Otorhinolaryngol 269:2401-9, 2012

321. Boeri M, Pastorino U, Sozzi G: Role of microRNAs in lung cancer: microRNA signatures in cancer prognosis. Cancer J 18:268-74, 2012

322. Andorfer CA, Necela BM, Thompson EA, et al: MicroRNA signatures: clinical biomarkers for the diagnosis and treatment of breast cancer. Trends Mol Med 17:313-9, 2011

323. Colombo PE, Milanezi F, Weigelt B, et al: Microarrays in the 2010s: the contribution of microarray-based gene expression profiling to breast cancer classification, prognostication and prediction. Breast Cancer Res 13:212, 2011

324. Pais H, Nicolas FE, Soond SM, et al: Analyzing mRNA expression identifies Smad3 as a microRNA-140 target regulated only at protein level. RNA 16:489-94, 2010

325. Butz H, Liko I, Czirjak S, et al: MicroRNA profile indicates downregulation of the TGFbeta pathway in sporadic non-functioning pituitary adenomas. Pituitary 14:112-24, 2011

326. Hermeking H: The miR-34 family in cancer and apoptosis. Cell Death Differ 17:193-9, 2010

327. Wang W, Peng B, Wang D, et al: Human tumor microRNA signatures derived from large-scale oligonucleotide microarray datasets. Int J Cancer 129:1624-34, 2011

328. Akervall J, Kurnit DM, Adams M, et al: Overexpression of cyclin D1 correlates with sensitivity to cisplatin in squamous cell carcinoma cell lines of the head and neck. Acta Otolaryngol 124:851-7, 2004

329. Pawlik TM, Keyomarsi K: Role of cell cycle in mediating sensitivity to radiotherapy. Int J Radiat Oncol Biol Phys 59:928-42, 2004 
330. Ma BB, Kam MK, Leung SF, et al: A phase II study of concurrent cetuximab-cisplatin and intensity-modulated radiotherapy in locoregionally advanced nasopharyngeal carcinoma. Ann Oncol 23:1287-92, 2012

331. Ma B, Hui EP, King A, et al: A phase II study of patients with metastatic or locoregionally recurrent nasopharyngeal carcinoma and evaluation of plasma Epstein-Barr virus DNA as a biomarker of efficacy. Cancer Chemother Pharmacol 62:59-64, 2008

332. Hattori Y, Fukushima M, Maitani Y: Non-viral delivery of the connexin 43 gene with histone deacetylase inhibitor to human nasopharyngeal tumor cells enhances gene expression and inhibits in vivo tumor growth. Int J Oncol 30:1427-39, 2007

333. Lo KW, Chung GT, To KF: Deciphering the molecular genetic basis of NPC through molecular, cytogenetic, and epigenetic approaches. Semin Cancer Biol 22:79-86, 2012

334. Wang Y, Moorhead M, Karlin-Neumann G, et al: Allele quantification using molecular inversion probes (MIP). Nucleic Acids Res 33:e183, 2005

335. Wang Y, Carlton VE, Karlin-Neumann G, et al: High quality copy number and genotype data from FFPE samples using Molecular Inversion Probe (MIP) microarrays. BMC Med Genomics 2:8, 2009

336. Wang Y, Cottman M, Schiffman JD: Molecular inversion probes: a novel microarray technology and its application in cancer research. Cancer Genet 205:341-55, 2012

337. Diskin SJ, Eck T, Greshock J, et al: STAC: A method for testing the significance of DNA copy number aberrations across multiple array-CGH experiments. Genome Res $16: 1149-58,2006$

338. Syampurnawati M, Tatsumi E, Furuta K, et al: HLA-DR-negative AML (M1 and M2): FLT3 mutations (ITD and D835) and cell-surface antigen expression. Leuk Res 31:921-9, 2007

339. Bianchini M, Ottaviani E, Grafone T, et al: Rapid detection of Flt3 mutations in acute myeloid leukemia patients by denaturing HPLC. Clin Chem 49:1642-50, 2003

340. Gumy-Pause F, Wacker P, Maillet P, et al: ATM alterations in childhood non-Hodgkin lymphoma. Cancer Genet Cytogenet 166:101-11, 2006

341. Fang NY, Greiner TC, Weisenburger DD, et al: Oligonucleotide microarrays demonstrate the highest frequency of ATM mutations in the mantle cell subtype of lymphoma. Proc Natl Acad Sci U S A 100:5372-7, 2003

342. Fang Y, Guan X, Guo Y, et al: Analysis of genetic alterations in primary nasopharyngeal carcinoma by comparative genomic hybridization. Genes Chromosomes Cancer 30:254-60, 2001 
343. Lu QL, Elia G, Lucas S, et al: Bcl-2 proto-oncogene expression in Epstein-Barr-virusassociated nasopharyngeal carcinoma. Int J Cancer 53:29-35, 1993

344. Coe BP, Ylstra B, Carvalho B, et al: Resolving the resolution of array CGH. Genomics $89: 647-53,2007$

345. Tuefferd M, De Bondt A, Van Den Wyngaert I, et al: Genome-wide copy number alterations detection in fresh frozen and matched FFPE samples using SNP 6.0 arrays. Genes Chromosomes Cancer 47:957-64, 2008

346. Fendri A, Kontos CK, Khabir A, et al: Quantitative analysis of BCL2 mRNA expression in nasopharyngeal carcinoma: an unfavorable and independent prognostic factor. Tumour Biol 31:391-9, 2010

347. He XX, Chang Y, Meng FY, et al: MicroRNA-375 targets AEG-1 in hepatocellular carcinoma and suppresses liver cancer cell growth in vitro and in vivo. Oncogene 31:335769,2012

348. Ward A, Balwierz A, Zhang JD, et al: Re-expression of microRNA-375 reverses both tamoxifen resistance and accompanying EMT-like properties in breast cancer. Oncogene $32: 1173-82,2013$

349. Isozaki Y, Hoshino I, Nohata N, et al: Identification of novel molecular targets regulated by tumor suppressive miR-375 induced by histone acetylation in esophageal squamous cell carcinoma. Int J Oncol 41:985-94, 2012

350. Yu C, Liu Y, Tan H, et al: Metadherin regulates metastasis of squamous cell carcinoma of the head and neck via AKT signalling pathway-mediated epithelialmesenchymal transition. Cancer Lett:[epub ahead of print], 2013

351. Zheng J, Li C, Wu X, et al: Astrocyte elevated gene-1 (AEG-1) shRNA sensitizes Huaier polysaccharide (HP)-induced anti-metastatic potency via inactivating downstream P13K/Akt pathway as well as augmenting cell-mediated immune response. Tumour Biol:[epub ahead of print], 2013

352. Long M, Hao M, Dong K, et al: AEG-1 overexpression is essential for maintenance of malignant state in human AML cells via up-regulation of Akt1 mediated by AURKA activation. Cell Signal 25:1438-46, 2013

353. Liu B, Wu Y, Peng D: Astrocyte elevated gene-1 regulates osteosarcoma cell invasion and chemoresistance via endothelin-1/endothelin A receptor signaling. Oncol Lett 5:505510,2013

354. Ke ZF, Mao X, Zeng C, et al: AEG-1 expression characteristics in human non-small cell lung cancer and its relationship with apoptosis. Med Oncol 30:383, 2013 
355. Ke ZF, He S, Li S, et al: Expression characteristics of astrocyte elevated gene-1 (AEG1) in tongue carcinoma and its correlation with poor prognosis. Cancer Epidemiol 37:17985,2013

356. Huang K, Li LA, Meng Y, et al: High expression of astrocyte elevated gene-1 (AEG1 ) is associated with progression of cervical intraepithelial neoplasia and unfavorable prognosis in cervical cancer. World J Surg Oncol 11:297, 2013

357. Li C, Chen K, Cai J, et al: Astrocyte elevated gene-1: a novel independent prognostic biomarker for metastatic ovarian tumors. Tumour Biol:[epub ahead of print], 2013

358. Yang Y, Kang P, Gao J, et al: AU-binding factor 1 expression was correlated with metadherin expression and progression of hepatocellular carcinoma. Tumour Biol:[epub ahead of print], 2013

359. Zheng J, Li C, Wu X, et al: Astrocyte elevated gene-1 is a novel biomarker of epithelial-mesenchymal transition and progression of hepatocellular carcinoma in two China regions. Tumour Biol:[epub ahead of print], 2013

360. Ahn S, Hyeon J, Park CK: Metadherin is a prognostic predictor of hepatocellular carcinoma after curative hepatectomy. Gut Liver 7:206-12, 2013

361. Erdem H, Oktay M, Yildirim U, et al: Expression of AEG-1 and p53 and their clinicopathological significance in malignant lesions of renal cell carcinomas: a microarray study. Pol J Pathol 64:28-32, 2013

362. Wang N, Du X, Zang L, et al: Prognostic impact of Metadherin-SND1 interaction in colon cancer. Mol Biol Rep 39:10497-504, 2012

363. Tokunaga E, Nakashima Y, Yamashita N, et al: Overexpression of metadherin/MTDH is associated with an aggressive phenotype and a poor prognosis in invasive breast cancer. Breast Cancer:[epub ahead of print], 2012

364. Zhou J, Li J, Wang Z, et al: Metadherin is a novel prognostic marker for bladder cancer progression and overall patient survival. Asia Pac J Clin Oncol 8:e42-8, 2012

365. Sun S, Ke Z, Wang F, et al: Overexpression of astrocyte-elevated gene-1 is closely correlated with poor prognosis in human non-small cell lung cancer and mediates its metastasis through up-regulation of matrix metalloproteinase- 9 expression. Hum Pathol 43:1051-60, 2012

366. Monni O, Joensuu H, Franssila K, et al: BCL2 overexpression associated with chromosomal amplification in diffuse large B-cell lymphoma. Blood 90:1168-74, 1997

367. Bali A, Bali D, Sharma A: An overview of gene therapy in head and neck cancer. Indian J Hum Genet 19:282-290, 2013 
368. Moll J: Target identification and validation in drug discovery : methods and protocols. New York, Springer, 2013

369. Lee SG, Kang DC, DeSalle R, et al: AEG-1/MTDH/LYRIC, the beginning: initial cloning, structure, expression profile, and regulation of expression. Adv Cancer Res 120:138,2013

370. Ma BB, Lui VW, Hui CW, et al: Preclinical evaluation of the mTOR-PI3K inhibitor BEZ235 in nasopharyngeal cancer models. Cancer Lett 343:24-32, 2014

371. Wong CH, Loong HH, Hui CW, et al: Preclinical evaluation of the PI3K-mTOR dual inhibitor PF-04691502 as a novel therapeutic drug in nasopharyngeal carcinoma. Invest New Drugs 31:1399-408, 2013

372. Yang F, Qian XJ, Qin W, et al: Dual phosphoinositide 3-kinase/mammalian target of rapamycin inhibitor NVP-BEZ235 has a therapeutic potential and sensitizes cisplatin in nasopharyngeal carcinoma. PLoS One 8:e59879, 2013

373. Smith PA, Merritt D, Barr L, et al: An orthotopic model of metastatic nasopharyngeal carcinoma and its application in elucidating a therapeutic target that inhibits metastasis. Genes Cancer 2:1023-33, 2011

374. Huang J, Manning BD: A complex interplay between Akt, TSC2 and the two mTOR complexes. Biochem Soc Trans 37:217-22, 2009

375. Erdem H, Yildirim U, Uzunlar AK, et al: Relationship among expression of basicfibroblast growth factor, MTDH/astrocyte elevated gene-1, adenomatous polyposis coli, matrix metalloproteinase 9, and COX-2 markers with prognostic factors in prostate carcinomas. Niger J Clin Pract 16:418-23, 2013

376. Yoo BK, Emdad L, Lee SG, et al: Astrocyte elevated gene-1 (AEG-1): A multifunctional regulator of normal and abnormal physiology. Pharmacol Ther 130:1-8, 2011

377. Lee AW, Tung SY, Chua DT, et al: Randomized trial of radiotherapy plus concurrentadjuvant chemotherapy vs radiotherapy alone for regionally advanced nasopharyngeal carcinoma. J Natl Cancer Inst 102:1188-98, 2010

378. Li XP, Li CY, Li X, et al: Inhibition of human nasopharyngeal carcinoma growth and metastasis in mice by adenovirus-associated virus-mediated expression of human endostatin. Mol Cancer Ther 5:1290-8, 2006 